\title{
Isotropic and Coisotropic Subvarieties of Grassmannians
}

\author{
vorgelegt von \\ M.Sc. Mathematik, M.Sc. Informatik \\ Kathlén Kohn \\ geb. in Rostock \\ von der Fakultät II - Mathematik und Naturwissenschaften \\ der Technischen Universität Berlin \\ zur Erlangung des akademischen Grades \\ Doktor der Naturwissenschaften \\ - Dr.rer.nat. - \\ genehmigte Dissertation
}

Promotionsausschuss:

Vorsitzender: Prof. Dr. Reinhold Schneider

Gutachter: Prof. Dr. Bernd Sturmfels

Gutachter: Prof. Dr. Peter Bürgisser

Gutachter: Prof. Dr. Ragni Piene

Tag der wissenschaftlichen Aussprache: 20. Juli 2018

Berlin 2018 



\section{Acknowledgements}

First of all, I want to express my gratitude towards my advisors Bernd Sturmfels and Peter Bürgisser. I am very happy about our open and trustful relationship. Both of you gave me a lot of support, mathematically and personally, so the German term Doktorvater really applies to both of you. Knowing Peter Bürgisser already since the second year of my Bachelor studies in Paderborn, I received a lot of education, advise and encouragement from his side. Teaching together was always fun, both in Paderborn and Berlin. I am thankful that Bernd Sturmfels gave me the chance to pursue a PhD in algebraic geometry, although I had almost no prior knowledge in this area. Your excitement about mathematics gave me inspiration and motivation to keep on learning and exploring, and I really enjoyed writing papers with you.

I want to thank my brilliant collaborators: Carlos Améndola, Ernst Ulrich Deuker, Pierre Lairez, Sara Lamboglia, Diane Maclagan, James Mathews, Bernt Ivar Utstøl Nødland, Ben Smith, Jeff Sommars, Matthew Trager, Paolo Tripoli and Magdalena Zajaczkowska. Many people supported this thesis by discussions of mathematical ideas, by reviewing some of the articles on which parts of this thesis are based, or by assistance with software: Daniele Agostini, Enrique Arrondo, Madeline Brandt, Dan Grayson, Anders Jensen, Michael Joswig, Lars Kastner, Antonio Lerario, Georg Loho, Emilia Mezzetti, Ragni Piene, Jean Ponce, Joachim Rieger, Emre Sertöz, Mike Stillman, Jenia Tevelev and Josephine Yu.

Special thanks to Ragni Piene for reviewing this thesis, supporting my applications with recommendation letters, and always being very friendly and welcoming and finding time to chat about math.

I would like to thank our Einstein team, including our postdocs Marta Panizzut, Fatemeh Mohammadi and Laura Escobar, as well as my academic brother Carlos Améndola. It was great to share an office with you and always fun to have game and movie nights. At this point I also want to thank Michael Joswig and his research group for welcoming our team on the sixth floor of the math building at TU Berlin. Special thanks to Antje Schulz, whose solution-oriented attitude made bureaucratic life at TU Berlin so much easier.

I want to express my gratitude towards Peter Bürgisser's research group: in particular, to Pierre Lairez for providing recommendation letters for my applications and to Jesko Hüttenhain for accompanying me throughout most of my scientific life. I acquired a great deal of my knowledge in algebraic geometry from Pierre and Jesko. Moreover, I want to thank my colleagues Paul Breiding, Josué Tonelli Cueto, Alperen Ergür and Mario Kummer, as well as our patient and friendly secretary Beate Nießen.

I am very grateful for the funding of the Einstein foundation and the grant BU 1371/2-2 of the Deutsche Forschungsgemeinschaft, without which this thesis would not have been possible. Furthermore, I want to thank the Berlin Mathematical School for providing Berlin with such a rich mathematical landscape and community. 
Special thanks to everyone who advised me in career and personal matters during my PhD time: among others, my BMS mentor Günter Ziegler, Timo de Wolff, Kristin Shaw, Diane Maclagan, Kaie Kubjas and Angelica Cueto. Moreover, I am thankful for the many friends in Team Haase and the Villa community at FU Berlin as well as in Bernd Sturmfels' groups in Berkeley and Leipzig.

I am more than thankful for all the people who encouraged and supported me on the way before I became a PhD student. This includes many professors from my time at the University of Paderborn, e.g., Johannes Blömer, Peter Bürgisser, Christian Fleischhack, Joachim Hilgert and Torsten Wedhorn. You inspired me to study math and computer science simultaneously and provided me with a lot of good advice, so without you I would have definitely not pursued PhD studies in mathematics. Furthermore, I want to thank the student council for math and computer science at the university of Paderborn and all my other nice fellow students, including Evgeni Wachnowezki, Malte Splietker, Juliana Seidlez, Tobias Rojahn, Alexander Dircksen and Tobias Black. Your positive feedback was a great source of motivation and brought me back on track whenever I had self-doubts. Without you I would not have become a PhD student at all.

Finally, I want to thank the people closest to my heart. My oldest and best friends Anita Möller, Franziska Pfennig and Felix Weißmann, for staying down to earth, believing in me and holding together over so many years. My verysoon-to-be husband Jonas Strandqvist, for adapting his life for my scientific career and always understanding phases with a high workload. My family, in particular my parents, for always having my back, helping wherever they can and supporting all my life decisions. 


\section{Abstract}

In this thesis, we study subvarieties of Grassmannians which are characterized by certain rank one conditions on their tangent or conormal spaces. Although these concepts seem to be very abstract at first sight, we will see that many of these subvarieties are naturally associated to underlying projective varieties. Typically such varieties consist of linear spaces which meet a projective variety with some prescribed contact. First examples are the set of all lines intersecting a space curve or the set of all lines tangent to a space curve.

A subvariety of a Grassmannian is called coisotropic if its conormal spaces satisfy the above mentioned rank one conditions. The notion of coisotropic hypersurfaces was introduced by Gel'fand, Kapranov and Zelevinsky. We develop their theory further and generalize their notion to subvarieties of Grassmannians with codimension higher than one. Moreover, we introduce the dual notion of isotropic varieties by requiring the rank one conditions to hold on their tangent spaces instead of their conormal spaces.

Throughout this thesis, we investigate different aspects of isotropic and coisotropic varieties. We focus on classifying them by their underlying projective varieties. Furthermore, we are interested in their degrees, their singular loci and their behavior under projective duality. We also provide Macaulay 2 code for explicit computations with some of the varieties we encounter in this thesis. In parts of this thesis, we restrict ourselves to the Grassmannian of lines in three-dimensional projective space, which contains already many interesting and non-trivial examples of (co)isotropic varieties. These lead us to questions which have been actively studied in the 19th century, like the classification of congruences, and to modern applications in algebraic vision, a recently emerged research area in the intersection of computer vision and algebraic geometry.

Additionally, we present two rather unrelated results at the end of this thesis, which have been developed in parallel to the findings described above. First, we describe a Macaulay2 package for computations in tropical geometry. Secondly, we investigate a simplicial complex whose facets represent the most widely used scales in western music. 



\section{Zusammenfassung}

In dieser Arbeit untersuchen wir Untervarietäten von Graßmann-Mannigfaltigkeiten, welche durch gewisse Rang-Eins-Bedingungen auf deren Tangentialoder Konormalräumen charakterisiert sind. Obwohl diese Konzepte auf den ersten Blick sehr abstrakt wirken, werden wir sehen, dass viele dieser Untervarietäten auf natürliche Weise zu zugrundeliegenden projektiven Varietäten assoziiert sind. Typischerweise bestehen solche Varietäten aus linearen Räumen, welche eine projektive Varietät mit einem vorgegebenen Kontakt treffen. Erste Beispiele hierfür sind die Menge aller Linien, die eine Raumkurve schneiden, oder die Menge aller Linien, die zu einer Raumkurve tangential sind.

Eine Untervarietät einer Graßmann-Mannigfaltigkeit heißt koisotrop, falls ihre Konormalenräume die oben genannten Rang-Eins-Bedingungen erfüllen. Der Begriff der koisotropen Hyperfläche wurde von Gel'fand, Kapranov und Zelevinsky eingeführt. Wir entwickeln diese Theorie weiter und verallgemeinern diesen Begriff auf Untervariatetäten von Graßmann-Mannigfaltigkeiten mit größerer Kodimension als eins. Weiterhin führen wir den dualen Begriff der isotropen Varietäten ein, indem wir verlangen, dass obige Rang-Eins-Bedingungen auf deren Tangential- statt deren Konormalräumen gelten.

Im Laufe dieser Arbeit studieren wir verschiedene Aspekte isotroper und koisotroper Varietäten. Ein Schwerpunkt ist die Klassifizierung dieser bezüglich ihrer zugrundeliegenden projektiven Varietäten. Außerdem interessieren wir uns für ihre Grade, ihre singulären Orten sowie ihr Verhalten unter projektiver Dualität. Wir stellen auch Macaulay2-Code für explizite Berechnungen mit einigen der Varietäten aus dieser Arbeit zur Verfügung. In Teilen dieser Arbeit beschränken wir uns auf die Graßmann-Mannigfaltigkeit der Linien im dreidimensionalen projektiven Raum, welche bereits viele interessante und nichttriviale Beispiele von (ko)isotropen Varietäten enthält. Diese führen uns zu Fragen, welche aktiv im 19. Jahrhundert untersucht wurden, wie zum Beispiel die Klassifizierung der algebraischen Strahlensysteme, sowie zu modernen Anwendungen in Algebraic Vision, einem vor Kurzem entstandenen Forschungsgebiet im Schnittbereich von algebraischer Geometrie und Computer Vision.

Zudem stellen wir zwei eher unverwandte Resultate am Ende dieser Arbeit vor, welche parallel zu den oben beschriebenen Ergebnissen entwickelt wurden: Erstens beschreiben wir ein Macaulay2-Paket für Berechnungen in der tropischen Geometrie und zweitens untersuchen wir einen Simplizialkomplex, dessen Facetten den meistverwendeten Skalen in der westlichen Musik entsprechen. 



\section{Declaration of Authorship}

I, Kathlén Kohn, declare that this thesis titled Isotropic and Coisotropic Subvarieties of Grassmannians and the work presented in it are my own or based on joint work of my own with co-authors. Parts of this thesis are prepublished.

- Chapter 3, except Sections 3.7 and 3.8, is based on the single-authored paper Coisotropic Hypersurfaces in Grassmannians [64]. A preprint is available at arXiv: 1607.05932.

- Section 3.7 is based on the joint paper Computing the Chow variety of quadratic space curves [13] with Peter Bürgisser, Pierre Lairez and Bernd Sturmfels. It was presented in Berlin at MACIS 2015 International Conference on Mathematical Aspects of Computer and Information Sciences and published as one of the revised selected papers.

- Chapter 5, except Section 5.6, is based on the joint paper Secants, bitangents, and their congruences [66] with Bernt Ivar Utstøl Nødland and Paolo Tripoli published as a chapter of the book Combinatorial Algebraic Geometry.

- Chapter 6 is based on the joint paper Changing Views on Curves and Surfaces [67] with Bernd Sturmfels and Matthew Trager published on Acta Mathematica Vietnamica.

- Chapter 8 is based on the joint paper Computing Tropical Varieties in Macaulay2 [2] with Carlos Améndola, Sara Lamboglia, Diane Maclagan, Ben Smith, Jeff Sommars, Paolo Tripoli and Magdalena Zajaczkowska. A preprint is available at arXiv: 1710.10651.

- Chapter 9 is based on the joint paper The Complex of Non-Chromatic Scales [65] with Ernst Ulrich Deuker. The German version of this article is published on Mitteilungen der DMV. A preprint of the English version is available at arXiv: 1710.05979. 



\section{Contents}

1 Introduction 1

1.1 Main Results . . . . . . . . . . . . . . . . . . . . . . . . . . . . 3

1.2 Additional Parts . . . . . . . . . . . . . . . . . . 4 4

\begin{tabular}{|lll}
\hline (Co)Isotropic Varieties & 5
\end{tabular}

\begin{tabular}{llll}
\hline 2 & Preliminaries & 7
\end{tabular}

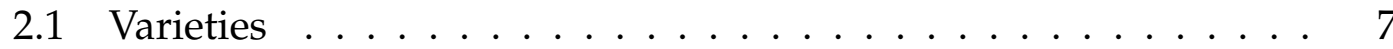

2.2 Grassmannians . . . . . . . . . . . . . . . . . 8

2.3 Segre Varieties . . . . . . . . . . . . . . . . . 16

$\begin{array}{lll}3 & \text { Coisotropic Hypersurfaces } & 19\end{array}$

3.1 Cayley Trick . . . . . . . . . . . . . . . . . . . . . . . . 20

3.2 Polar Degrees . . . . . . . . . . . . . . . . . . . . . . 22

3.3 Rank One Characterization . . . . . . . . . . . . . . . . . 25

3.4 Duality . . . . . . . . . . . . . . . . . 26

3.5 Hyperdeterminants . . . . . . . . . . . . . . . . . . . . . 29

3.6 Coordinate Systems of Grassmannians . . . . . . . . . . . . . . 31

3.7 Cayley Variety $\ldots \ldots \ldots \ldots \ldots$

3.8 Transitive Action on Tangent Spaces . . . . . . . . . . . . . . . 41

3.9 Computations $\ldots \ldots \ldots \ldots \ldots \ldots \ldots \ldots \ldots \ldots$

4 Coisotropic Varieties 45

4.1 Grassmann secant varieties . . . . . . . . . . . . . . . 45

4.2 Two Notions of Coisotropy $\ldots \ldots \ldots$. . . . . . . . . . . . . 46

4.3 Strongly Coisotropic Varieties . . . . . . . . . . . . . . . . . . . . . 49

4.4 Lines with Higher Contact to Hypersurfaces . . . . . . . . . . . 54

5 Singular Loci of Coisotropic Hypersurfaces in $\operatorname{Gr}\left(1, \mathbb{P}^{3}\right) \quad \mathbf{6 1}$

5.1 Secants of Space Curves $\ldots \ldots \ldots \ldots \ldots$. . . . . . . . . 63

5.2 Bitangents and Inflections of Hypersurfaces . . . . . . . . . . . 67

5.3 Projective Duality . . . . . . . . . . . . . . . . . . . . . . 69

5.4 Intersection Theory . . . . . . . . . . . . . . . . . . . . 75

5.5 Singular Loci of Congruences . . . . . . . . . . . . . . . . . . 79

5.6 Tangent Spaces to Congruences . . . . . . . . . . . . . . . . . . 84

\begin{tabular}{|lll}
6 & Changing Views on Curves and Surfaces & 89
\end{tabular}

6.1 Ruled Surfaces and Developable Surfaces . . . . . . . . . . . . . 91 
6.2 Views of Curves . . . . . . . . . . . . . . . . . . . . . 97

6.3 Views of Surfaces . . . . . . . . . . . . . . . . . . . 107

6.4 Intersection Theory . . . . . . . . . . . . . . . . . . . . . . . . . 112

6.5 Computing Visual Events . . . . . . . . . . . . . . . 118

\begin{tabular}{llr}
\hline 7 & Isotropic Varieties & $\mathbf{1 2 5}$
\end{tabular}

7.1 Isotropic Curves . . . . . . . . . . . . . . . . . . . . . . . . . . . . . . 126

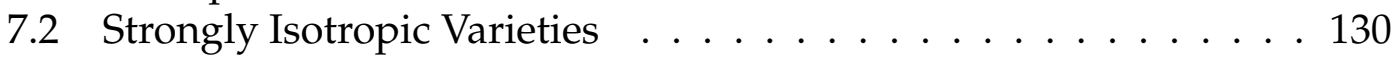

7.3 Congruences . . . . . . . . . . . . . . . . 133

II A Package for Tropical Geometry 143

8 Computing Tropical Varieties in Macaulay2 $\mathbf{1 4 5}$

8.1 Mathematical Background . . . . . . . . . . . . . . . . . . . . . 145

8.2 Examples . . . . . . . . . . . . . . . . . . . . . . . . 146

8.3 Future Plans . . . . . . . . . . . . . . . . . . . . . . . . . 149

III A Simplicial Complex for Music Theory and Praxis 151

9 The Complex of Non-Chromatic Scales 153

9.1 f-Vector $\ldots \ldots \ldots \ldots \ldots \ldots$

9.2 Facets . . . . . . . . . . . . . . . . . . . . . . . . . . . . . . . . . . . . . . . . . . . . . . . . . . . . . . . .

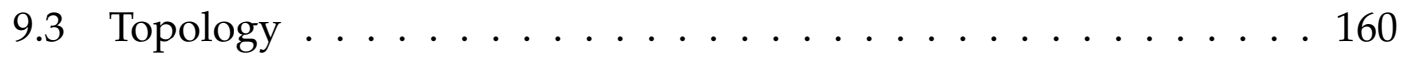

\begin{tabular}{ll}
\hline Appendix & 169
\end{tabular}

\begin{tabular}{ll}
\hline Bibliography & 181
\end{tabular}

\begin{tabular}{ll}
\hline List of Figures & 189
\end{tabular}

\begin{tabular}{ll}
\hline List of Tables & 191
\end{tabular}

\begin{tabular}{ll}
\hline List of Symbols & 193
\end{tabular} 


\section{Introduction}

The parametrization of all subvarieties of $\mathbb{P}^{n}$ with fixed dimension and degree is one of the most important problems in the history of algebraic geometry. The parametrization of zero-dimensional varieties is trivial. Hypersurfaces can be parametrized by their defining equations. Linear subspaces of $\mathbb{P}^{n}$ are points in their respective Grassmannian. Hence, the first non-trivial case are curves in $\mathbb{P}^{3}$ of degree (at least) two.

Space curves can be parametrized by their Chow forms, which were first introduced by Cayley [18]. The Chow hypersurface of a curve $C \subset \mathbb{P}^{3}$ is the set of all lines in $\mathbb{P}^{3}$ that intersect $C$. This is a hypersurface in the Grassmannian $\operatorname{Gr}\left(1, \mathbb{P}^{3}\right)$ of lines in $\mathbb{P}^{3}$. It is defined by one equation in the Plücker coordinates of $\operatorname{Gr}\left(1, \mathbb{P}^{3}\right)$, which is unique up to a constant factor and the Plücker relation. This polynomial has the same degree as the curve $C$ and is called the Chow form of $C$. One can recover the vanishing ideal of the curve $C$ from its Chow form. Thus, the variety of Chow forms with a fixed degree in the coordinate ring of the Grassmannian $\operatorname{Gr}\left(1, \mathbb{P}^{3}\right)$ is a parameter space for the set of all space curves with that fixed degree. Moreover, although the vanishing ideal of a space curve is defined by at least two polynomials, which are clearly not unique, the Chow form is a single equation defining the curve.

Example 1.1 ([28, Prop. 1.2]). The twisted cubic is a smooth rational curve of degree three in $\mathbb{P}^{3}$. Parametrically, this curve is the image of the morphism $v_{3}: \mathbb{P}^{1} \rightarrow \mathbb{P}^{3}$ defined by $(s: t) \mapsto\left(s^{3}: s^{2} t: s t^{2}: t^{3}\right)$. Its vanishing ideal is minimally generated by three quadrics: $x_{0} x_{3}-x_{1} x_{2}, x_{1}^{2}-x_{0} x_{2}, x_{2}^{2}-x_{1} x_{3}$. The line $L$, which is determined by the two equations $a_{0} x_{0}+a_{1} x_{1}+a_{2} x_{2}+a_{3} x_{3}=0$ and $b_{0} x_{0}+b_{1} x_{1}+b_{2} x_{2}+b_{3} x_{3}=0$, intersects the twisted cubic if and only if there exists a point $(s: t) \in \mathbb{P}^{1}$ such that

$$
a_{0} s^{3}+a_{1} s^{2} t+a_{2} s t^{2}+a_{3} t^{3}=0=b_{0} s^{3}+b_{1} s^{2} t+b_{2} s t^{2}+b_{3} t^{3} .
$$

The resultant for these two cubic polynomials, which can be expressed as the determinant of their Sylvester matrix, vanishes exactly when they have a common root. It follows that the line $L$ meets the twisted cubic if and only if

$$
0=\operatorname{det}\left[\begin{array}{cccccc}
a_{0} & a_{1} & a_{2} & a_{3} & 0 & 0 \\
0 & a_{0} & a_{1} & a_{2} & a_{3} & 0 \\
0 & 0 & a_{0} & a_{1} & a_{2} & a_{3} \\
b_{0} & b_{1} & b_{2} & b_{3} & 0 & 0 \\
0 & b_{0} & b_{1} & b_{2} & b_{3} & 0 \\
0 & 0 & b_{0} & b_{1} & b_{2} & b_{3}
\end{array}\right]=-\operatorname{det}\left[\begin{array}{ccc}
p_{01} & p_{02} & p_{03} \\
p_{02} & p_{03}+p_{12} & p_{13} \\
p_{03} & p_{13} & p_{23}
\end{array}\right],
$$


where $p_{i j}$ are the Plücker coordinates of $L$, i.e., the minors of $\left[\begin{array}{llll}a_{0} & a_{1} & a_{2} & a_{3} \\ b_{0} & b_{1} & b_{2} & b_{3}\end{array}\right]$. Hence, the Chow form of the twisted cubic is

$$
p_{03}^{3}+p_{03}^{2} p_{12}-2 p_{02} p_{03} p_{13}+p_{01} p_{13}^{2}+p_{02}^{2} p_{23}-p_{01} p_{03} p_{23}-p_{01} p_{12} p_{23} \text {. }
$$

This polynomial is uniquely determined, up to a constant factor and the Plücker relation $p_{01} p_{23}-p_{02} p_{13}+p_{03} p_{12}=0$.

The generalization of Chow forms to arbitrary varieties was given by Chow and van der Waerden [23]. For a given variety $X \subset \mathbb{P}^{n}$ of dimension $k$, projective subspaces of dimension $n-k-1$ have typically no intersection with the variety $X$, but those subspaces that do intersect $X$ form the Chow hypersurface of $X$ in the corresponding Grassmannian $\operatorname{Gr}\left(n-k-1, \mathbb{P}^{n}\right)$. The Chow form of $X$ is the defining polynomial of this hypersurface, which is a unique (up to scaling with constants) polynomial in the coordinate ring of $\operatorname{Gr}\left(n-k-1, \mathbb{P}^{n}\right)$. It has the same degree as $X$ and determines $X$ uniquely. Therefore, the variety of Chow forms with a fixed degree in the coordinate ring of the Grassmannian $\operatorname{Gr}\left(n-k-1, \mathbb{P}^{n}\right)$ is a parameter space for the set of all $k$-dimensional subvarieties $X \subset \mathbb{P}^{n}$ with that fixed degree. For more on Chow forms, see [28].

This parameter space is called Chow variety by Gel'fand, Kapranov and Zelevinsky [43, Ch. 4]. A natural question is to describe the vanishing ideal of such a Chow variety, which was already posed by Green and Morrison [46]. Their approach to this problem is also discussed in [43, Ch. 4.3]. Here, the authors proceed in two steps. First, they note that the vectors in the conormal spaces of a Chow hypersurface are homomorphisms of rank at most one. They call all hypersurfaces of Grassmannians with this rank-one-property coisotropic and they give criteria to determine if a given hypersurface is coisotropic. Secondly, following [46], they describe how to distinguish the Chow forms among all coisotropic forms.

Cayley had already realized that a hypersurface in the Grassmannian $\operatorname{Gr}\left(1, \mathbb{P}^{3}\right)$ of lines in $\mathbb{P}^{3}$ is coisotropic if and only if it is either the Chow hypersurface of a space curve or the variety formed by all tangent lines to a surface in $\mathbb{P}^{3}$. A generalization of the latter type of coisotropic hypersurfaces is studied by Sturmfels in [101]: given a subvariety $X \subset \mathbb{P}^{n}$ of dimension $k$, the Hurwitz hypersurface of $X$ is the subvariety of the Grassmannian $\operatorname{Gr}\left(n-k, \mathbb{P}^{n}\right)$ consisting of all $(n-k)$-dimensional subspaces which do not intersect $X$ in $\operatorname{deg}(X)$ reduced points. So shortly put, Cayley's result says that a hypersurface of $\operatorname{Gr}\left(1, \mathbb{P}^{3}\right)$ is coisotropic if and only if it is either the Chow hypersurface of a curve or the Hurwitz hypersurface of a surface. Recently, Catanese [16] showed that these are exactly the self-dual hypersurfaces of $\operatorname{Gr}\left(1, \mathbb{P}^{3}\right)$. Moreover, the volume of the Hurwitz hypersurface of a projective variety $X$ plays a crucial role in the study of the condition of intersecting $X$ with varying linear subspaces [12]. More generally, one can compute the volume of all coisotropic hypersurfaces, which is essential for the probabilistic Schubert calculus proposed by Bürgisser and Lerario [14].

An important generalization of Cayley's result is presented in [43, Ch. 4, 
Thm. 3.14], which gives a geometric characterization of all coisotropic hypersurfaces. Given a subvariety $X \subset \mathbb{P}^{n}$, the higher associated hypersurfaces of $X$ are subvarieties of Grassmannians formed by all linear spaces which intersect $X$ non-transversely. For example, Chow and Hurwitz hypersurfaces are higher associated hypersurfaces. Gel'fand, Kapranov and Zelevinsky show in [43, Ch. 4, Thm. 3.14] that a hypersurface of a Grassmannian is coisotropic if and only if it is a higher associated hypersurface of some projective variety.

\subsection{Main Results}

The starting point of this thesis is the described main result [43, Ch. 4, Thm. 3.14] by Gel'fand, Kapranov and Zelevinsky about coisotropic hypersurfaces. We aim to study these hypersurfaces in more detail and to generalize the theory presented in [43]. The study of subvarieties of Grassmannians is a journey from 19th century algebraic geometry, where the geometry of linear spaces was an active area of research (e.g., see [94, 55, 33]), to recently emerged subjects like algebraic vision, which is the interplay of computer vision and algebraic geometry. The main difference of this thesis compared to other literature is the focus on the tangent spaces of subvarieties of Grassmannians and the geometric results derived from these. This new point of view yields many interesting insights.

First of all, we give a new proof for [43, Ch. 4, Thm. 3.14] in Chapter 3. Furthermore, we study the degrees of coisotropic hypersurfaces and their relation to projective duality, since the latter is one of the key concepts throughout the whole thesis. We will also present the topic from a computational point of view by providing a Macaulay2 package for calculations with coisotropic hypersurfaces.

In Chapter 4, we introduce two notions which generalize coisotropy to subvarieties of Grassmannians with larger codimension. For the stronger of the two notions, we provide a full geometric characterization, which is a generalization of [43, Ch. 4, Thm. 3.14]. For the weaker notion, we discuss non-trivial and geometrically meaningful examples; namely varieties formed by all lines which meet a given hypersurface with higher contact.

We study iterated singular loci of the coisotropic hypersurfaces of $\operatorname{Gr}\left(1, \mathbb{P}^{3}\right)$ in Chapter 5. Moreover, we explicitly describe the tangent spaces of the singular loci with dimension two. An application of these results to algebraic vision is provided in Chapter 6, where we study the visual event surface of a given curve or surface in $\mathbb{P}^{3}$.

Finally, in Chapter 7, we introduce a dual notion to coisotropy. Instead of requiring rank-one-conditions on the vectors in the conormal spaces of a subvariety of a Grassmannian, we impose those conditions on its tangent vectors. The notion corresponding to a coisotropic hypersurface is then an isotropic curve. We give a full classification of all isotropic curves. Furthermore, we present two notions which generalize isotropy to larger dimension; these notions correspond 
to the two notions of coisotropy mentioned above. For the stronger of the two notions, we provide again a full geometric characterization. The weaker notion is, for example, satisfied by all surfaces in $\operatorname{Gr}\left(1, \mathbb{P}^{3}\right)$. Using this, we present a geometric classification of all surfaces in $\operatorname{Gr}\left(1, \mathbb{P}^{3}\right)$. This has been partly known in classical literature [94], but our focus on the geometry of tangent spaces gives a full and formal proof.

\subsection{Additional Parts}

Part I of this thesis contains the main results discussed above. In Part II and III. we present additional results which are less related with the main content.

Following the computational focus of some of the chapters in Part I, we describe a Macaulay2 package for computations with tropical varieties in Chapter 8. In tropical geometry, we can associate to each algebraic variety a polyhedral complex. This combinatorial objects reflects parts of the geometry of the original variety. More generally, tropical geometry studies polyhedral complexes which do not arise from algebraic varieties but have the same properties as those complexes that do arise like this. These are called tropical varieties. The purpose of the package presented in Chapter 8 is to facilitate computations in tropical geometry using Macaulay2. It provides a powerful and user friendly tool for computing tropical varieties requiring little prerequisite knowledge and making the process as simple as possible for the end user.

Chapter 9 lies in the interplay between mathematics and music. We describe a simplicial complex capturing all commonly used musical scales, and explicitly compute its facets and topology. These results are not only relevant for musical theory. Instead they are mainly motivated by improvisational practice. Moreover, since Chapter 9 targets both mathematicians and musicians, it is written in an elementary manner, such that mathematical concepts are explained for musicians and the other way around. 


\section{Part I}

\section{(Co)Isotropic Varieties}





\section{Preliminaries}

We usually work over an algebraically closed field of characteristic zero, unless otherwise specified. All vector spaces are finite-dimensional. The dual vector space of a given vector space $V$ is denoted by $V^{*}$. We denote by $\mathbb{A}^{n+1}$ affine $(n+1)$-dimensional space and by $\mathbb{P}^{n}:=\mathbb{P}\left(\mathbb{A}^{n+1}\right)$ its projectivization. Similarly, we write $\left(\mathbb{P}^{n}\right)^{*}:=\mathbb{P}\left(\left(\mathbb{A}^{n+1}\right)^{*}\right)$. The List of Symbols at the end of this thesis should help the reader to keep track of the notation.

\subsection{Varieties}

All algebraic varieties that appear in this thesis are either affine or projective. By an affine variety in $\mathbb{A}^{n+1}$ we mean the common zero locus of some polynomials in $n+1$ variables. Similarly, a projective variety in $\mathbb{P}^{n}$ is the common zero locus of homogeneous polynomials in $n+1$ variables. In particular, varieties do not necessarily have to be irreducible. We say that an affine or projective variety is nondegenerate if it spans its ambient space.

Given a set $P$ of polynomials in $n+1$ variables, we write $Z(P)$ for their common zero locus in $\mathbb{A}^{n+1}$. If the ideal generated by $P$ is homogeneous, $Z(P)$ usually denotes the zero locus in $\mathbb{P}^{n}$. Vice versa, given a subset $S$ of $\mathbb{A}^{n+1}$ or $\mathbb{P}^{n}$, we write $I(S)$ for its vanishing ideal in $n+1$ variables. Moreover, $\bar{S}:=Z(I(S))$ denotes the Zariski closure of $S$.

We denote the affine cone over a subset $S \subset \mathbb{P}^{n}$ by $S \subset \mathbb{A}^{n+1}$. The projectivization of an affine cone $\mathcal{C}$ is denoted by $\mathbb{P}(\mathcal{C})$. We write $\operatorname{Sing}(X)$ for the singular locus of a variety $X$ and $\operatorname{Reg}(X):=X \backslash \operatorname{Sing}(X)$ for its regular locus. If $X \subset \mathbb{P}^{n}$, the embedded tangent space of $X$ at $p \in \operatorname{Reg}(X)$ is

$$
\mathbb{T}_{X, p}:=\left\{y \in \mathbb{P}^{n} \mid \forall f \in I(X): \sum_{i=0}^{n} \frac{\partial f}{\partial x_{i}}(p) \cdot y_{i}=0\right\}
$$

A hyperplane in $\mathbb{P}^{n}$ is called tangent to $X$ at a smooth point $p$ if it contains $\mathbb{T}_{X, p}$. Note that each hyperplane $H \subset \mathbb{P}^{n}$ corresponds to a point in $\left(\mathbb{P}^{n}\right)^{*}$, which we denote by $H^{\vee}$. The projectively dual variety of $X$ is

$$
X^{\vee}:=\overline{\left\{H^{\vee} \mid H \subset \mathbb{P}^{n} \text { hyperplane, } \exists p \in \operatorname{Reg}(X): \mathbb{T}_{X, p} \subset H\right\}} \subset\left(\mathbb{P}^{n}\right)^{*} .
$$

If $X$ is irreducible, so is $X^{\vee}$ [43, Ch. 1, Prop. 1.3]. For example, if $L$ is an $\ell$ dimensional projective subspace of $\mathbb{P}^{n}$, then $L^{\vee}$ is a projective subspace of $\left(\mathbb{P}^{n}\right)^{*}$ with dimension $n-\ell-1$, whose points correspond to hyperplanes contain- 
ing $L$. In particular, we have $\left(\mathbb{P}^{n}\right)^{\vee}=\varnothing$. Throughout this thesis, we use the convention that the empty set is a projective space with dimension -1 .

Example 2.1. The dual of a line in $\mathbb{P}^{2}$ is a point, and the dual of an irreducible plane curve of degree at least two is again a plane curve. The dual of a line in $\mathbb{P}^{3}$ is a line, and the dual of an irreducible curve in $\mathbb{P}^{3}$ of degree at least two is a surface. The dual of a plane in $\mathbb{P}^{3}$ is a point, and the dual of an irreducible surface in $\mathbb{P}^{3}$ of degree at least two can be either a curve or a surface.

We will make frequent use of the following biduality of projective varieties, which is also known as reflexivity.

Theorem 2.2 (Biduality theorem [43, Ch. 1, Thm. 1.1]). Let $X \subset \mathbb{P}^{n}$ be a projective variety over an algebraically closed field of characteristic zero. If $p \in \operatorname{Reg}(X)$ and $H^{\vee} \in \operatorname{Reg}\left(X^{\vee}\right)$, then the hyperplane $H$ is tangent to $X$ at the point $p$ if and only if the hyperplane $p^{\vee}$ is tangent to $X^{\vee}$ at the point $H^{\vee}$. In particular, we have $\left(X^{\vee}\right)^{\vee}=X$.

We note that Gel'fand, Kapranov and Zelevinsky prove the biduality theorem in [43] over the ground field $\mathbb{C}$ of complex numbers. Due to Lefschet $z^{\prime}$ principle [72, 6, 36, 39, 96], this extends to any algebraically closed field of characteristic zero. We invoke this principle occasionally throughout this thesis, without mentioning it explicitly.

In the remainder of this chapter, we discuss the most important examples of projective varieties for this thesis: Grassmannians and Segre varieties.

\subsection{Grassmannians}

The Grassmannian $\operatorname{Gr}\left(\ell, \mathbb{P}^{n}\right)$ is the set of all projective subspaces of $\mathbb{P}^{n}$ with dimension $\ell$. It is naturally identified with the Grassmannian $\operatorname{Gr}\left(\ell+1, \mathbb{A}^{n+1}\right)$ of $(\ell+1)$-dimensional linear subspaces of $\mathbb{A}^{n+1}$. Moreover, it is a $(\ell+1)(n-\ell)$ dimensional variety, embedded in $\mathbb{P}^{\left(\begin{array}{c}n+1 \\ \ell+1\end{array}\right)-1}$ via Plücker coordinates.

Coordinate systems. We now describe six different coordinate systems on the Grassmannian $\operatorname{Gr}\left(\ell+1, \mathbb{A}^{n+1}\right)$. Following the conventions in [43, Ch. 3.1] and [101], we call these primal/dual Plücker/Stiefel/affine coordinates.

First, let $A \in \mathbb{A}^{(n-\ell) \times(n+1)}$ be a matrix such that $L \in \operatorname{Gr}\left(\ell+1, \mathbb{A}^{n+1}\right)$ is the kernel of $A$. The entries of $A$ are primal Stiefel coordinates of $L$, and the maximal minors $p_{i_{1} \ldots i_{n-\ell}}$ of $A$ are the primal Plïcker coordinates of L. Up to scaling, the Plücker coordinates are unique, whereas the Stiefel coordinates are clearly not: multiplying $A$ with any invertible $(n-\ell) \times(n-\ell)$-matrix does not change its kernel. Hence, when denoting by $S(n-\ell, n+1)$ the Stiefel manifold of all $(n-\ell) \times(n+1)$-matrices of full rank, the Grassmannian $\operatorname{Gr}\left(\ell+1, \mathbb{A}^{n+1}\right)$ can be seen as the quotient $S(n-\ell, n+1) / \mathrm{GL}(n-\ell)$. 
Pick now a maximal linearly independent subset of the columns of $A$, indexed by $\left(i_{1}, \ldots, i_{n-\ell}\right)$, and multiply the inverse of this submatrix with $A$ itself. The resulting matrix has the same kernel as $A$ and its columns indexed by $\left(i_{1}, \ldots, i_{n-\ell}\right)$ form the identity matrix. The remaining entries of this new matrix are the primal affine coordinates of $L$. These give a unique representation of $L$ in the primal affine chart

$$
\begin{aligned}
U_{i_{1} \ldots i_{n-\ell}}: & =\left\{\begin{array}{l|l}
\operatorname{ker} A \mid \begin{array}{l}
i_{j} \text {-th column of } A \in \mathbb{A}^{(n-\ell) \times(n+1)} \text { is the } \\
\text { standard basis vector } e_{j}(\text { for } j=1, \ldots, n-\ell)
\end{array}
\end{array}\right\} \\
& =\left\{L \in \operatorname{Gr}\left(\ell+1, \mathbb{A}^{n+1}\right) \mid p_{i_{1} \ldots i_{n-\ell}}(L) \neq 0\right\} \cong \mathbb{A}^{(n-\ell) \times(\ell+1)}
\end{aligned}
$$

of the Grassmannian $\operatorname{Gr}\left(\ell+1, \mathbb{A}^{n+1}\right)$.

Secondly, let $B \in \mathbb{A}^{(\ell+1) \times(n+1)}$ such that $L$ is the row space of $B$. The entries of $B$ are dual Stiefel coordinates of $L$, and the maximal minors $q_{j_{0} \ldots j_{\ell}}$ of $B$ are the dual Plücker coordinates of $L$. As above, for every maximal linearly independent subset of columns of $B$, indexed by $\left(j_{0}, \ldots, j_{\ell}\right)$, the subspace $L$ has unique dual affine coordinates in the dual affine chart $\left\{L \mid q_{j_{0} \ldots j_{\ell}}(L) \neq 0\right\} \cong \mathbb{A}^{(\ell+1) \times(n-\ell)}$ of the Grassmannian $\operatorname{Gr}\left(\ell+1, \mathbb{A}^{n+1}\right)$.

There is a canonical isomorphism

$$
\begin{aligned}
\operatorname{Gr}\left(\ell, \mathbb{P}^{n}\right) & \stackrel{\sim}{\longrightarrow} \operatorname{Gr}\left(n-\ell-1,\left(\mathbb{P}^{n}\right)^{*}\right), \\
L & \longmapsto L^{\vee} .
\end{aligned}
$$

Primal coordinates on $\operatorname{Gr}\left(\ell, \mathbb{P}^{n}\right)$ are dual coordinates on $\operatorname{Gr}\left(n-\ell-1,\left(\mathbb{P}^{n}\right)^{*}\right)$, and vice versa. To see this, we write $L \in \operatorname{Gr}\left(\ell, \mathbb{P}^{n}\right)$ as the projectivization of the kernel of a homomorphism $\varphi \in \operatorname{Hom}\left(\mathbb{A}^{n+1}, \mathbb{A}^{n-\ell}\right)$, which corresponds to a matrix $A \in \mathbb{A}^{n-\ell} \otimes\left(\mathbb{A}^{n+1}\right)^{*}$. The entries of $A$ are primal Stiefel coordinates of $L$. We consider the natural identifications

$$
\begin{aligned}
& \operatorname{Hom}\left(\mathbb{A}^{n+1}, \mathbb{A}^{n-\ell}\right) \cong \mathbb{A}^{n-\ell} \otimes\left(\mathbb{A}^{n+1}\right)^{*} \cong\left(\mathbb{A}^{n+1}\right)^{*} \otimes \mathbb{A}^{n-\ell} \cong \operatorname{Hom}\left(\left(\mathbb{A}^{n-\ell}\right)^{*},\left(\mathbb{A}^{n+1}\right)^{*}\right), \\
& \varphi \quad \mapsto \quad A \quad \mapsto \quad A^{T} \quad \mapsto \quad \varphi^{*},
\end{aligned}
$$

where $\varphi^{*}:\left(\mathbb{A}^{n-\ell}\right)^{*} \rightarrow\left(\mathbb{A}^{n+1}\right)^{*}, f \mapsto f \circ \varphi$ denotes the dual homomorphism of $\varphi$. Note that $L^{\vee}$ is the projectivization of the image of $\varphi^{*}$. Hence, the entries of $A^{T}$ are dual Stiefel coordinates for $L^{\vee}$.

To change between primal and dual coordinates on the same Grassmannian (which coincides by the above observation with the map (2.2) using the same coordinates), we write $L$ as the projectivization of the kernel of $\left(I_{n-\ell} \mid M\right)$, where $I_{n-\ell}$ denotes the $(n-\ell)$-dimensional identity matrix and $M$ is some $(n-\ell) \times$ $(\ell+1)$-matrix. In this case, $L$ is the projectivized row space of $\left(-M^{T} \mid I_{\ell+1}\right)$. The analogous statement holds for the other affine charts of $\operatorname{Gr}\left(\ell, \mathbb{P}^{n}\right)$. For the 
Plücker coordinates it follows that

$$
q_{j_{0} \ldots j_{\ell}}=s\left(i_{1}, \ldots, i_{n-\ell}\right) \cdot p_{i_{1} \ldots i_{n-\ell^{\prime}}}
$$

where $i_{1}, \ldots, i_{n-\ell}$ form the complement of $\left\{j_{0}, \ldots, j_{\ell}\right\}$ in strictly increasing order and $s\left(i_{1}, \ldots, i_{n-\ell}\right)$ denotes the sign of the permutation $\left(i_{1}, \ldots, i_{n-\ell}, j_{0}, \ldots, j_{\ell}\right)$.

Example 2.3. Consider the following polynomial in the primal Plücker coordinates of $\operatorname{Gr}\left(1, \mathbb{P}^{3}\right)$, which will appear again in Examples 3.6 and 3.16 .

$$
\begin{aligned}
& \left(p_{01}^{6}+p_{02}^{6}+p_{03}^{6}+p_{12}^{6}+p_{13}^{6}+p_{23}^{6}\right) \\
+ & 2\left(p_{10}^{3} p_{02}^{3}+p_{10}^{3} p_{03}^{3}+p_{20}^{3} p_{03}^{3}+p_{01}^{3} p_{12}^{3}+p_{01}^{3} p_{13}^{3}+p_{21}^{3} p_{13}^{3}\right) \\
+ & 2\left(p_{02}^{3} p_{21}^{3}+p_{02}^{3} p_{23}^{3}+p_{12}^{3} p_{23}^{3}+p_{03}^{3} p_{31}^{3}+p_{03}^{3} p_{32}^{3}+p_{13}^{3} p_{32}^{3}\right) \\
+ & 2\left(p_{01} p_{23}\left(p_{03}^{2} p_{12}^{2}-p_{02}^{2} p_{13}^{2}\right)-p_{02} p_{13}\left(p_{01}^{2} p_{23}^{2}-p_{03}^{2} p_{12}^{2}\right)+p_{03} p_{12}\left(p_{02}^{2} p_{13}^{2}-p_{01}^{2} p_{23}^{2}\right)\right) .
\end{aligned}
$$

To display the symmetry of the polynomial, the convention $p_{j i}=-p_{i j}$ for $i<j$ is used. The change of coordinates

$$
p_{01} \mapsto q_{23}, p_{02} \mapsto-q_{13}, p_{03} \mapsto q_{12}, p_{12} \mapsto q_{03}, p_{13} \mapsto-q_{02}, p_{23} \mapsto q_{01},
$$

yields the polynomial in dual Plücker coordinates:

$$
\begin{aligned}
& \left(q_{01}^{6}+q_{02}^{6}+q_{03}^{6}+q_{12}^{6}+q_{13}^{6}+q_{23}^{6}\right) \\
- & 2\left(q_{10}^{3} q_{02}^{3}+q_{10}^{3} q_{03}^{3}+q_{20}^{3} q_{03}^{3}+q_{01}^{3} q_{12}^{3}+q_{01}^{3} q_{13}^{3}+q_{21}^{3} q_{13}^{3}\right) \\
- & 2\left(q_{02}^{3} q_{21}^{3}+q_{02}^{3} q_{23}^{3}+q_{12}^{3} q_{23}^{3}+q_{03}^{3} q_{31}^{3}+q_{03}^{3} q_{32}^{3}+q_{13}^{3} q_{32}^{3}\right) \\
+ & 2\left(q_{01} q_{23}\left(q_{03}^{2} q_{12}^{2}-q_{02}^{2} q_{13}^{2}\right)-q_{02} q_{13}\left(q_{01}^{2} q_{23}^{2}-q_{03}^{2} q_{12}^{2}\right)+q_{03} q_{12}\left(q_{02}^{2} q_{13}^{2}-q_{01}^{2} q_{23}^{2}\right)\right) .
\end{aligned}
$$

The polynomial in primal or dual Stiefel coordinates is obtained by substituting the $2 \times 2$-minor given by the columns $i$ and $j$ of a general $2 \times 4$-matrix $\left[\begin{array}{llll}a_{0} & a_{1} & a_{2} & a_{3} \\ b_{0} & b_{1} & b_{2} & b_{3}\end{array}\right]$ into $p_{i j}$ or $q_{i j}$, respectively. Similarly, one gets the polynomial in primal or dual affine coordinates by using a matrix of the form $\left[\begin{array}{llll}1 & 0 & a_{2} & a_{3} \\ 0 & 1 & b_{2} & b_{3}\end{array}\right]$, e.g., by substituting

$$
q_{01} \mapsto 1, q_{02} \mapsto b_{2}, q_{03} \mapsto b_{3}, q_{12} \mapsto-a_{2}, q_{13} \mapsto-a_{3}, q_{23} \mapsto a_{2} b_{3}-b_{2} a_{3} .
$$

Remark 2.4 ([43, Ch. 3, Prop. 2.1]). Every hypersurface in $\operatorname{Gr}\left(\ell, \mathbb{P}^{n}\right)$ is defined by one homogeneous polynomial in Plücker coordinates, which is unique up to a constant factor and the Plücker relations of $\operatorname{Gr}\left(\ell, \mathbb{P}^{n}\right)$. The Plücker relations are the polynomials in the vanishing ideal of the image of $\operatorname{Gr}\left(\ell, \mathbb{P}^{n}\right)$ under its Plücker embedding into $\mathbb{P}^{\left(\begin{array}{c}n+1 \\ \ell+1\end{array}\right)-1}$.

Tangent and conormal spaces. Grassmannians are smooth and irreducible varieties. The tangent space of $\operatorname{Gr}\left(\ell+1, \mathbb{A}^{n+1}\right)$ at a point $L \in \operatorname{Gr}\left(\ell+1, \mathbb{A}^{n+1}\right)$ 
is naturally identified with

$$
T_{\mathrm{Gr}\left(\ell+1, \mathbb{A}^{n+1}\right), L}:=\operatorname{Hom}\left(L, \mathbb{A}^{n+1} / L\right) .
$$

A detailed discussion of tangent spaces of Grassmannians can for example be found in [49, Lecture 16]. In fact, the description in (2.8) is very intuitive. A tangent vector at $L$ is a direction in which $L$ can move. Such a direction can be specified by describing the movement of every point on the linear subspace $L$, i.e., by a homomorphism $L \rightarrow \mathbb{A}^{n+1}$. If the direction of movement of a point on $L$ lies inside $L$, its movement does not contribute to the direction of movement of $L$. Thus, a tangent vector of $\operatorname{Gr}\left(\ell+1, \mathbb{A}^{n+1}\right)$ at $L$ is already given by a homomorphism $\varphi: L \rightarrow \mathbb{A}^{n+1} / L$.

Remark 2.5. Since $T_{\mathrm{Gr}\left(\ell, \mathbb{P}^{n}\right), L}=\operatorname{Hom}\left(\boldsymbol{L}, \mathbb{A}^{n+1} / \boldsymbol{L}\right)$, the tangent space of projective space at a point $p \in \mathbb{P}^{n}$ is

$$
T_{\mathbb{P}^{n}, p}=\operatorname{Hom}\left(\boldsymbol{p}, \mathbb{A}^{n+1} / \boldsymbol{p}\right) \cong \mathbb{A}^{n+1} / \boldsymbol{p}
$$

Hence, for a projective variety $X \subset \mathbb{P}^{n}$, we distinguish between the Zariski tangent space $T_{X, p} \subset T_{\mathbb{P}^{n}, p}$ at $p \in \operatorname{Reg}(X)$ and the embedded tangent space $\mathbb{T}_{X, p} \subset \mathbb{P}^{n}$; see (2.1). Note that the dimension of both tangent spaces is $\operatorname{dim}(X)$, although the embedded tangent space is a projective space, whereas the Zariski tangent space is an abstract vector space. For an affine variety $Y \subset \mathbb{A}^{n+1}$ both notions coincide and we simply write $T_{Y, p} \subset \mathbb{A}^{n+1}$ for $p \in \operatorname{Reg}(Y)$. Note that $T_{X, p} \cong T_{\boldsymbol{X}, x} / \boldsymbol{p}$ and $\mathbb{T}_{X, p}=\mathbb{P}\left(T_{\boldsymbol{X}, x}\right)$ for $x \in \boldsymbol{p} \backslash\{0\}$.

For a subvariety $\Sigma \subset \operatorname{Gr}\left(\ell+1, \mathbb{A}^{n+1}\right)$, the normal space of $\Sigma$ at $L \in \operatorname{Reg}(\Sigma)$ is $N_{\Sigma, L}:=T_{\operatorname{Gr}\left(\ell+1, \mathbb{A}^{n+1}\right), L} / T_{\Sigma, L}$. Its dual vector space is the conormal space of $\Sigma$ at $L$. For two vector spaces $U$ and $W$, we identify the dual vector space of $\operatorname{Hom}(U, W)$ with $\operatorname{Hom}(W, U)$ via

$$
\begin{aligned}
\operatorname{Hom}(W, U) & \longrightarrow \operatorname{Hom}(U, W)^{*}, \\
\phi & \longmapsto \operatorname{tr}(\cdot \circ \phi)=\operatorname{tr}(\phi \circ \cdot),
\end{aligned}
$$

which we use to express the conormal space of $\Sigma$ at $L$ :

$$
\begin{aligned}
\left(N_{\Sigma, L}\right)^{*} & \cong\left\{\varphi \in\left(T_{\operatorname{Gr}\left(\ell+1, \mathbb{A}^{n+1}\right), L}\right)^{*} \mid T_{\Sigma, L} \subset \operatorname{ker} \varphi\right\} \\
& \cong\left\{\varphi \in \operatorname{Hom}\left(\mathbb{A}^{n+1} / L, L\right) \mid \forall \psi \in T_{\Sigma, L}: \operatorname{tr}(\varphi \circ \psi)=0\right\} \\
& =: N_{\Sigma, L}^{*}
\end{aligned}
$$

Remark/Definition 2.6. The canonical isomorphism (2.2) of Grassmannians can also be expressed as follows:

$$
\begin{aligned}
\operatorname{Gr}\left(\ell+1, \mathbb{A}^{n+1}\right) & \stackrel{\sim}{\longrightarrow} \operatorname{Gr}\left(n-\ell,\left(\mathbb{A}^{n+1}\right)^{*}\right), \\
U & \longmapsto\left(\mathbb{A}^{n+1} / U\right)^{*} .
\end{aligned}
$$


The differential of this isomorphism at $U \in \operatorname{Gr}\left(\ell+1, \mathbb{A}^{n+1}\right)$ is

$$
\begin{aligned}
\operatorname{Hom}\left(U, \mathbb{A}^{n+1} / U\right) & \longrightarrow \operatorname{Hom}\left(\left(\mathbb{A}^{n+1} / U\right)^{*}, U^{*}\right), \\
\varphi & \longmapsto \varphi^{*}
\end{aligned}
$$

For a subvariety $\Sigma \subset \operatorname{Gr}\left(\ell+1, \mathbb{A}^{n+1}\right)$, we denote by $\Sigma^{\perp} \subset \operatorname{Gr}\left(n-\ell,\left(\mathbb{A}^{n+1}\right)^{*}\right)$ the image of $\Sigma$ under this isomorphism. Since the trace of an endomorphism $\psi$ is zero if and only if the trace of $\psi^{*}$ is zero, the isomorphism of the conormal space of $\Sigma$ at $U \in \operatorname{Reg}(\Sigma)$ with the corresponding conormal space of $\Sigma^{\perp}$ is

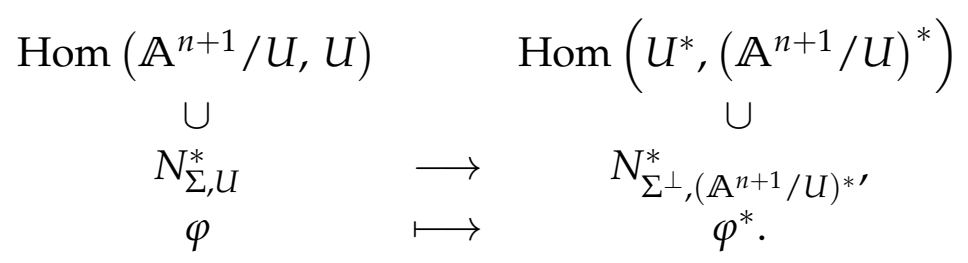

In this thesis, we will often consider incidence correspondences of Grassmannians and their tangent spaces. For this, we introduce the following notation. Let $\varphi: U \rightarrow V_{1} / V_{2}$ be a homomorphism of vector spaces, where $V_{2} \subset V_{1}$. For a subspace $W \subset U$, we denote by $\left.\varphi\right|_{W}: W \rightarrow V_{1} / V_{2}$ the restriction of $\varphi$ to $W$. Furthermore, for a vector space $V$ with $V_{2} \subset V \subset V_{1}$ we denote by $(\varphi \bmod V): U \rightarrow V_{1} / V$ the composition of the canonical projection $V_{1} / V_{2} \rightarrow V_{1} / V$ with $\varphi$.

Lemma 2.7. Let $k<\ell$. The tangent space of the flag variety

$$
F_{k, \ell}:=\left\{\left(L_{1}, L_{2}\right) \in \operatorname{Gr}\left(k+1, \mathbb{A}^{n+1}\right) \times \operatorname{Gr}\left(\ell+1, \mathbb{A}^{n+1}\right) \mid L_{1} \subset L_{2}\right\}
$$

at a point $\left(L_{1}, L_{2}\right) \in F_{k, \ell}$ is

$$
\begin{aligned}
T_{F_{k, \ell}\left(L_{1}, L_{2}\right)} & =\left\{(\psi, \varphi)|\varphi|_{L_{1}}=\left(\psi \bmod L_{2}\right)\right\} \\
& \subset \operatorname{Hom}\left(L_{1}, \mathbb{A}^{n+1} / L_{1}\right) \times \operatorname{Hom}\left(L_{2}, \mathbb{A}^{n+1} / L_{2}\right) .
\end{aligned}
$$

Proof. From our description of tangent spaces to Grassmannians it follows immediately that every tangent vector $(\psi, \varphi) \in T_{F_{k, \ell}\left(L_{1}, L_{2}\right)}$ and every $v \in L_{1}$ satisfy $\varphi(v)=\psi(v)$ modulo $L_{2}$; see also [49, Example 16.2, Exercise 16.3]. Hence, $T_{F_{k, \ell},\left(L_{1}, L_{2}\right)} \subset\left\{(\psi, \varphi)|\varphi|_{L_{1}}=\left(\psi \bmod L_{2}\right)\right\}$. Since both vector spaces have the same dimension, they are equal.

We usually apply this result to graphs of rational maps which are contained in $F_{k, \ell}$. Therefore, we formulate this version of the result explicitly in Corollary 2.9. For a rational map $\omega: X \rightarrow Y$ between varieties, we denote by $D_{p} \omega: T_{X, p} \rightarrow T_{Y, \omega(p)}$ the differential of $\omega$ at $p$ where it is defined.

Example 2.8. Many proofs in this thesis will follow the ideas presented in this example. 
Let $C \subset \mathbb{P}^{3}$ be an irreducible curve of degree at least two. The graph of the rational map

$$
\begin{aligned}
\omega: C & \rightarrow \operatorname{Gr}\left(1, \mathbb{P}^{3}\right), \\
\operatorname{Reg}(C) \ni p & \longmapsto \mathbb{T}_{C, p}
\end{aligned}
$$

is contained in the flag variety $F_{0,1}$. We denote the Zariski closure of the image of $\omega$ by $\mathcal{T}^{\ell}(C)$; here we follow the notational convention of Chapter 6 , where the upper index " $\ell$ " stands for lines, since points in $\mathcal{T}^{\ell}(C)$ correspond to tangent lines of $C$. For a general point $p$ on the curve $C$ with tangent line $L:=\mathbb{T}_{C, p}$, the differential $D_{p} \mathcal{\omega}: T_{C, p} \rightarrow T_{\mathcal{T}^{\ell}(C), L}$ is an isomorphism. Note that

$$
T_{C, p}=\left\{\varphi \in \operatorname{Hom}\left(\boldsymbol{p}, \mathbb{A}^{4} / \boldsymbol{p}\right) \mid \operatorname{im} \varphi \subset \boldsymbol{L} / \boldsymbol{p}\right\} \text {. }
$$

By the following Corollary 2.9. we have for every $\varphi \in T_{C, p}$ that

$$
\left.D_{p} \omega(\varphi)\right|_{p}=(\varphi \bmod \boldsymbol{L})
$$

Since the image of every $\varphi \in T_{C, p}$ is contained in $L / p$ and $D_{p} \omega$ is an isomorphism, the kernel of every homomorphism in $T_{\mathcal{T}^{\ell}(C), L} \subset \operatorname{Hom}\left(\boldsymbol{L}, \mathbb{A}^{4} / \boldsymbol{L}\right)$ contains $\boldsymbol{p}$. In particular, the one-dimensional vector space $T_{\mathcal{T} \ell(C), L}$ is spanned by a homomorphism $\psi: L \rightarrow \mathbb{A}^{4} / \boldsymbol{L}$ such that $\boldsymbol{p} \subset \operatorname{ker} \psi$. Since $\boldsymbol{L}$ is a plane containing the line $\boldsymbol{p}$, the kernel of $\psi$ is equal to $\boldsymbol{p}$ and the homomorphism $\psi$ has rank one. We will see in Section 7.1 that the one-dimensional image of this homomorphism is $H / L$, where $H \subset \mathbb{P}^{3}$ is the osculating plane of the curve $C$ at the point $p$.

Corollary 2.9. Let $\Sigma \subset \operatorname{Gr}\left(\ell+1, \mathbb{A}^{n+1}\right)$ be an irreducible subvariety with a rational map $\omega: \Sigma \rightarrow \operatorname{Gr}\left(k+1, \mathbb{A}^{n+1}\right)$. If $k \leq \ell$ and $\omega(L) \subset L$ for a general $L \in \Sigma$, then a general $L \in \Sigma$ satisfies

$$
\left.\varphi\right|_{\mathscr{\omega}(L)}=\left(D_{L} \mathcal{\omega}(\varphi) \bmod L\right)
$$

for every $\varphi \in T_{\Sigma, L}$, i.e., the following diagram commutes:

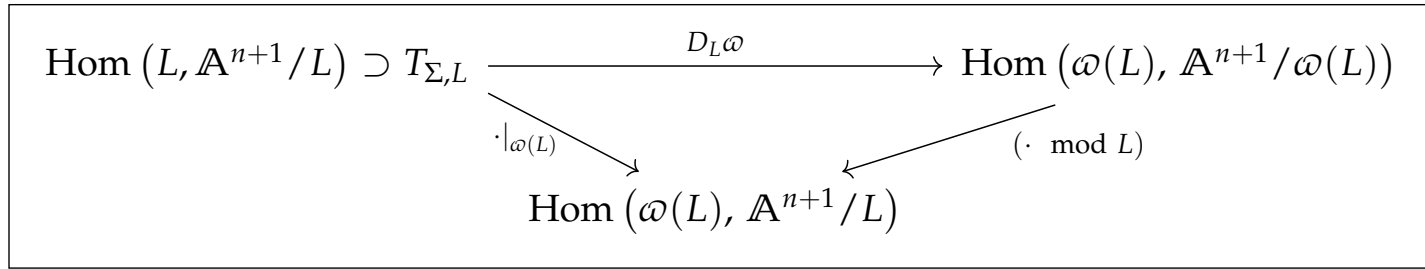

If $k \geq \ell$ and $\omega(L) \supset L$ for a general $L \in \Sigma$, then a general $L \in \sum$ satisfies

$$
\left.D_{L} \omega(\varphi)\right|_{L}=(\varphi \bmod \omega(L))
$$

for every $\varphi \in T_{\Sigma, L}$, i.e., the following diagram commutes: 


$$
\operatorname{Hom}\left(L, \mathbb{A}^{n+1} / L\right) \supset T_{\Sigma, L} \underset{\operatorname{Hom}\left(L, \mathbb{A}^{n+1} / \omega(L)\right)}{\bmod \omega(L))} \operatorname{Hom}\left(\omega(L), \mathbb{A}_{\cdot_{L}}^{n+1} / \omega(L)\right)
$$

Proof. We denote by $\Sigma_{0} \subset \Sigma$ the subset where $\omega$ is defined. Let us first assume that $k \leq \ell$ and that $\omega(L) \subset L$ holds for all $L \in \Sigma_{0}$. We consider the Zariski closure of the graph of $\omega$ :

$$
\Gamma:=\overline{\left\{(\mathcal{\omega}(L), L) \mid L \in \Sigma_{0}\right\}} \subset\left(\operatorname{Gr}\left(k+1, \mathbb{A}^{n+1}\right) \times \Sigma\right) \cap F_{k, \ell}
$$

Moreover, let $\pi_{1}: \Gamma \rightarrow \operatorname{Gr}\left(k+1, \mathbb{A}^{n+1}\right)$ and $\pi_{2}: \Gamma \rightarrow \Sigma$ denote the projections onto the first and second factor, respectively. We can draw another commutative diagram (see below): at a general $L \in \Sigma$, the differential $D_{(\omega(L), L)} \pi_{2}$ is bijective and $D_{L} \mathcal{\omega}=D_{(\omega(L), L)} \pi_{1} \circ\left(D_{(\omega(L), L)} \pi_{2}\right)^{-1}$. Hence, by Lemma 2.7, the differential $D_{L} \mathcal{\omega}$ of $\omega$ at $L$ satisfies (2.9).

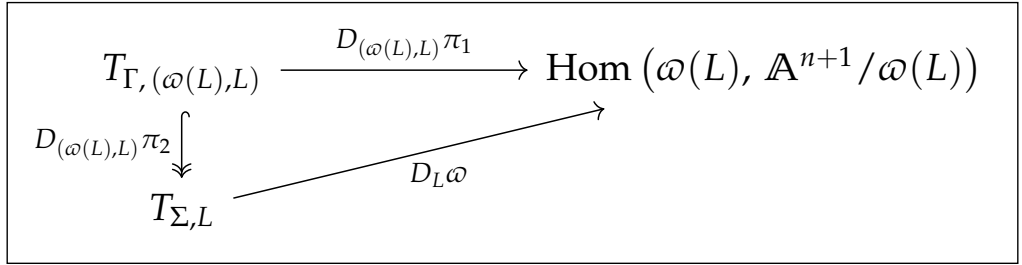

If $k \geq \ell$ and $L \subset \mathcal{\omega}(L)$ for all $L \in \Sigma_{0}$, we proceed analogously.

With Lemma 2.7 we can also understand the differential of the rational map

$$
\begin{aligned}
\rho: \mathbb{A}^{(\ell+1) \times(n+1)} & \longrightarrow \operatorname{Gr}\left(\ell+1, \mathbb{A}^{n+1}\right), \\
A & \longmapsto \operatorname{rowspace}(A) .
\end{aligned}
$$

For an $(\ell+1) \times(n+1)$-matrix $A$ and $0 \leq i \leq \ell$, we write $A_{i} \in \mathbb{A}^{n+1}$ for the $i$-th row of $A$.

Corollary 2.10. Let $A \in \mathbb{A}^{(\ell+1) \times(n+1)}$ be a matrix of full rank and let $L:=\rho(A)$ denote its rowspace. The differential of (2.10) at $A$ is given by

$$
\begin{aligned}
D_{A} \rho: \mathbb{A}^{(\ell+1) \times(n+1)} & \longrightarrow \operatorname{Hom}\left(L, \mathbb{A}^{n+1} / L\right), \\
M & \longmapsto\left(A_{i} \mapsto M_{i}+L \quad \text { for all } 0 \leq i \leq \ell\right) .
\end{aligned}
$$

Proof. Let $i \in\{0, \ldots, \ell\}$. We consider the map

$$
\begin{aligned}
\pi_{i}: \mathbb{A}^{(\ell+1) \times(n+1)} & \longrightarrow \operatorname{Gr}\left(1, \mathbb{A}^{n+1}\right) \cong \mathbb{P}^{n}, \\
A & \longmapsto \operatorname{span}\left(A_{i}\right) .
\end{aligned}
$$


Its differential at $A$ is

$$
\begin{aligned}
D_{A} \pi_{i}: \mathbb{A}^{(\ell+1) \times(n+1)} & \longrightarrow \operatorname{Hom}\left(\operatorname{span}\left(A_{i}\right), \mathbb{A}^{n+1} / \operatorname{span}\left(A_{i}\right)\right), \\
M & \longmapsto\left(A_{i} \mapsto M_{i}+\operatorname{span}\left(A_{i}\right)\right) .
\end{aligned}
$$

The differential of the product map $\pi_{i} \times \rho: \mathbb{A}^{(\ell+1) \times(n+1)} \rightarrow-\rightarrow F_{0, \ell}$ at $A$ is equal to $D_{A} \pi_{i} \times D_{A} \rho$. Hence, by Lemma 2.7 , we have for every $M \in \mathbb{A}^{(\ell+1) \times(n+1)}$ that

$$
\left(D_{A} \rho(M)\right)\left(A_{i}\right)=\left(D_{A} \pi_{i}(M) \bmod L\right)\left(A_{i}\right)=M_{i}+L .
$$

With this we can also describe the differential of the Plücker embedding

$$
\mathrm{pl}: \operatorname{Gr}\left(\ell+1, \mathbb{A}^{n+1}\right) \hookrightarrow \mathbb{P}\left(\bigwedge_{\ell+1} \mathbb{A}^{n+1}\right) .
$$

Recall that $\left(\bigwedge^{\ell+1} \mathbb{A}^{n+1}\right) / \operatorname{pl}(L)$ is naturally identified with the Zariski tangent space of $\mathbb{P}\left(\Lambda^{\ell+1} \mathbb{A}^{n+1}\right)$ at $\operatorname{pl}(L)$, where $\mathbf{p l}(L)$ denotes the one-dimensional linear space corresponding to the projective point $\mathrm{pl}(L)$.

Corollary 2.11. Let $L \in \operatorname{Gr}\left(\ell+1, \mathbb{A}^{n+1}\right)$ be the rowspace of a full rank matrix $A \in \mathbb{A}^{(\ell+1) \times(n+1)}$. For $\varphi \in T_{\mathrm{Gr}\left(\ell+1, \mathbb{A}^{n+1}\right), L}$ and $M_{0}, \ldots, M_{\ell} \in \mathbb{A}^{n+1}$ such that $\varphi\left(A_{i}\right)=M_{i}+L$ for $0 \leq i \leq \ell$, we have that

$$
\begin{aligned}
D_{L} \mathrm{pl}: \operatorname{Hom}\left(L, \mathbb{A}^{n+1} / L\right) & \longrightarrow\left(\bigwedge^{\ell+1} \mathbb{A}^{n+1}\right) / \mathbf{p l}(L) \\
\varphi & \longmapsto \sum_{i=0}^{\ell} A_{0} \wedge \ldots \wedge A_{i-1} \wedge M_{i} \wedge A_{i+1} \wedge \ldots \wedge A_{\ell}+\mathbf{p l}(L) .
\end{aligned}
$$

Proof. First note that this description of $\left(D_{L} \mathrm{pl}\right)(\varphi)$ is indeed well-defined, i.e., independent of the choice of $A$ and $M_{0}, \ldots, M_{\ell}$. Secondly, we consider the rational map

$$
\begin{aligned}
\mu: \mathbb{A}^{(\ell+1) \times(n+1)} & \longrightarrow \mathbb{P}\left(\bigwedge \mathbb{A}^{n+1}\right), \\
B & \longmapsto\left[B_{0} \wedge \ldots \wedge B_{\ell}\right],
\end{aligned}
$$

where $[x]:=\mathbb{P}(\operatorname{span}(x))$ denotes the projective point corresponding to a point $x$ in affine space. The differential of this map at $A$ is

$$
D_{A} \mu: \mathbb{A}^{(\ell+1) \times(n+1)} \longrightarrow\left(\bigwedge^{\ell+1} \mathbb{A}^{n+1}\right) / \boldsymbol{\mu}(A),
$$




$$
N \longmapsto \sum_{i=0}^{\ell} A_{0} \wedge \ldots \wedge A_{i-1} \wedge N_{i} \wedge A_{i+1} \wedge \ldots \wedge A_{\ell}+\boldsymbol{\mu}(\boldsymbol{A}) .
$$

Since $\mu=\mathrm{pl} \circ \rho$, Corollary 2.10 implies Corollary 2.11.

\subsection{Segre Varieties}

Since the elements of the tangent and conormal spaces of subvarieties of Grassmannians are homomorphisms, we can consider their rank. In this thesis, homomorphisms of rank one play a central role. For two vector spaces $U$ and $W$, we denote by $\operatorname{Seg}(U, W)$ the projectivization of $\{\varphi \in \operatorname{Hom}(U, W) \mid \operatorname{rank} \varphi \leq 1\}$. This Segre variety has dimension $d+e$ and degree $\left(\begin{array}{c}d+e \\ d\end{array}\right)$, where $d:=\operatorname{dim} U-1$ and $e:=\operatorname{dim} W-1$. It has two rulings by maximal projective subspaces: one ruling consists of the projectivizations of all $\alpha$-spaces, and the other ruling of the projectivizations of all $\beta$-spaces, which are defined as follows.

Definition 2.12. Let $U$ and $W$ be vector spaces. For a linear hyperplane $u \subset U$, we define the $\alpha$-space of $u$ as

$$
E_{\alpha}(u):=\{\varphi \in \operatorname{Hom}(U, W) \mid u \subset \operatorname{ker} \varphi\} .
$$

Analogously, the $\beta$-space of a one-dimensional linear subspace $w \subset W$ is

$$
E_{\beta}(w):=\{\varphi \in \operatorname{Hom}(U, W) \mid \operatorname{im} \varphi \subset w\} .
$$

Example 2.13. Let $U:=\mathbb{C}^{4}$ and $W:=\mathbb{C}^{3}$. We pick bases $\left(e_{1}, \ldots, e_{4}\right)$ and $\left(f_{1}, \ldots, f_{3}\right)$ of $U$ and $W$, respectively. With respect to these bases we identify $\operatorname{Hom}(U, W)$ with $\mathbb{C}^{3 \times 4}$. For $u:=\operatorname{span}\left\{e_{1}, e_{2}, e_{3}\right\}$ and $w:=\operatorname{span}\left\{f_{1}\right\}$, we have that $E_{\alpha}(u)$ and $E_{\beta}(w)$ consist of all matrices of the form

$$
\left[\begin{array}{llll}
0 & 0 & 0 & * \\
0 & 0 & 0 & * \\
0 & 0 & 0 & *
\end{array}\right] \quad \text { resp. } \quad\left[\begin{array}{cccc}
* & * & * & * \\
0 & 0 & 0 & 0 \\
0 & 0 & 0 & 0
\end{array}\right] .
$$

Remark 2.14. The isomorphism

$$
\begin{aligned}
\operatorname{Hom}(U, W) & \longrightarrow \operatorname{Hom}\left(W^{*}, U^{*}\right), \\
\varphi & \longmapsto \varphi^{*}
\end{aligned}
$$

maps the $\alpha$-space $E_{\alpha}(u)$ to the $\beta$-space $E_{\beta}\left((U / u)^{*}\right)$. Dually, it maps the $\beta$-space $E_{\beta}(w)$ to the $\alpha$-space $E_{\alpha}\left((W / w)^{*}\right)$.

Note that the notions rank, kernel and image are well-defined for elements in $\mathbb{P}(\operatorname{Hom}(U, W))$. Thus, for $\varphi \in \mathbb{P}(\operatorname{Hom}(U, W))$, we will usually write rank $\varphi$, $\operatorname{ker} \varphi$ and $\operatorname{im} \varphi$. For example, we write

$\operatorname{Seg}(U, W)=\{\varphi \in \mathbb{P}(\operatorname{Hom}(U, W)) \mid \operatorname{rank} \varphi=1\}$ is ruled by spaces of the form 
$\mathbb{P}\left(E_{\alpha}(u)\right)=\{\varphi \in \operatorname{Seg}(U, W) \mid \operatorname{ker} \varphi=u\}$ of dimension $e$ and $\mathbb{P}\left(E_{\beta}(w)\right)=\{\varphi \in \operatorname{Seg}(U, W) \mid \operatorname{im} \varphi=w\}$ of dimension $d$.

Now that we have discussed our basic notions and necessary tools, we begin to study coisotropic hypersurfaces of Grassmannians. 



\section{Coisotropic Hypersurfaces}

In this chapter, we revisit the main results in [43, Ch. 3+4] about coisotropic hypersurfaces and develop the theory further. We use the definition in [43, Def. 3.9]:

Definition 3.1. A hypersurface $\Sigma \subset \operatorname{Gr}\left(\ell, \mathbb{P}^{n}\right)$ is called coisotropic if, for every $L \in \operatorname{Reg}(\Sigma)$, the conormal space $N_{\Sigma, L}^{*} \subset \operatorname{Hom}\left(\mathbb{A}^{n+1} / L, L\right)$ is spanned by a homomorphism of rank one.

Moreover, in [43, Ch. 3, Sec. 2.E], Gel'fand, Kapranov and Zelevinsky define the higher associated hypersurfaces of projective varieties. Their main result about these hypersurfaces is that these are exactly the coisotropic ones [43, Ch. 4, Thm. 3.14]. Due to this result, we will use a more meaningful name for our main objects of study and call the $i$-th higher associated hypersurface of a projective variety $X$ the $i$-th coisotropic hypersurface of $X$. More specifically, we define:

Definition 3.2. Let $X \subset \mathbb{P}^{n}$ be an irreducible variety. For $i \in\{0, \ldots, \operatorname{dim} X\}$, the $i$-th coisotropic variety of $X$ is defined as

$$
\begin{aligned}
\mathrm{CH}_{i}(X): & =\overline{\left\{L \mid \exists x \in \operatorname{Reg}(X) \cap L: \operatorname{dim}\left(L \cap \mathbb{T}_{X, x}\right) \geq i\right\}} \\
& \subset \operatorname{Gr}\left(n-\operatorname{dim} X+i-1, \mathbb{P}^{n}\right) .
\end{aligned}
$$

If $\mathrm{CH}_{i}(X)$ has codimension one, we call it the $i$-th coisotropic hypersurface of $X$.

Note that the condition $\operatorname{dim}\left(L \cap \mathbb{T}_{X, x}\right) \geq i$ is equivalent to $\operatorname{dim}\left(L+\mathbb{T}_{X, x}\right)<n$, which means that $L$ intersects $X$ at $x$ non-transversely.

Example 3.3. $\mathrm{CH}_{0}(X)$ is the Chow hypersurface of $X$. If $\operatorname{deg} X \geq 2$, then $\mathrm{CH}_{1}(X)$ has codimension one in its ambient Grassmannian and is called the Hurwitz hypersurface of $X$ [101].

In Section 3.1, we determine for which index $i$ Definition 3.2 yields indeed a hypersurface in its ambient Grassmannian. We do this by showing that the coisotropic varieties of a projective variety parametrize projectively dual varieties to Segre products of the variety with projective spaces. We prove in Section 3.2 that the degrees of the coisotropic hypersurfaces of a projective variety coincide with its polar degrees.

Section 3.3 revisits the main theorem of [43] about coisotropic hypersurfaces, which states that the two above definitions of coisotropic hypersurfaces agree. In contrast to Definition 3.2. note that Definition 3.1 is local and does not depend on any underlying projective variety. The proof in [43] uses machinery from 
Lagrangian varieties to construct the projective variety $X$ a given coisotropic hypersurface is associated to, whereas our proof (Theorem 3.11) is more direct and explicit in defining the underlying variety $X$.

In Section 3.4, we derive that the coisotropic hypersurfaces of the projectively dual variety $X^{v}$ are the coisotropic hypersurfaces of $X$ in reversed order. Hyperdeterminants are special cases of coisotropic hypersurfaces, as we discuss in Section 3.5. We study characterizations of coisotropy in different coordinate systems on Grassmannians in Section 3.6 .

We define the Cayley variety as the set of all coisotropic hypersurfaces of fixed degree in a fixed ambient Grassmannian. We explicitly compute the vanishing ideal of the Cayley variety of coisotropic quadrics in $\operatorname{Gr}\left(1, \mathbb{P}^{3}\right)$ in Section 3.7 . Moreover, we compute the vanishing ideal of its Chow subvariety, i.e., the variety of all quadratic Chow hypersurfaces in $\operatorname{Gr}\left(1, \mathbb{P}^{3}\right)$.

The unitary group acts transitively on the tangent spaces of a coisotropic hypersurface, which allows to compute volumes of intersections of general translates of coisotropic hypersurfaces under the group action, see [14]. This is essential for the probabilistic Schubert calculus proposed in [14]. We show in Section 3.8 that not all hypersurfaces of Grassmannians with such an action are coisotropic. Finally, we present a Macaulay2 package for computations with coisotropic hypersurfaces in Section 3.9

\subsection{Cayley Trick}

The Chow hypersurface can be constructed as the dual of a Segre product, which is known as the Cayley trick [43, Ch. 3, Thm. 2.7]. We generalize this for all coisotropic varieties. For an irreducible variety $X \subset \mathbb{P}^{n}$ of dimension $k$ and an integer $0 \leq i \leq k$, we consider the Segre embedding

$$
\begin{aligned}
& X \times\left(\mathbb{P}^{k-i}\right)^{*} \hookrightarrow \mathbb{P}^{n} \times\left(\mathbb{P}^{k-i}\right)^{*} \hookrightarrow \mathbb{P}\left(\mathbb{A}^{n+1} \otimes\left(\mathbb{A}^{k-i+1}\right)^{*}\right)=\mathbb{P}\left(\operatorname{Hom}\left(\mathbb{A}^{k-i+1}, \mathbb{A}^{n+1}\right)\right) \\
& \text { and }\left(X \times\left(\mathbb{P}^{k-i}\right)^{*}\right)^{\vee} \hookrightarrow \mathbb{P}\left(\operatorname{Hom}\left(\mathbb{A}^{n+1}, \mathbb{A}^{k-i+1}\right)\right) .
\end{aligned}
$$

With this, we will show that the projectively dual variety $\left(X \times\left(\mathbb{P}^{k-i}\right)^{*}\right)^{\vee}$ is in fact $\mathrm{CH}_{i}(X)$, when both varieties are interpreted in the primal Stiefel space $\mathbb{P}\left(\operatorname{Hom}\left(\mathbb{A}^{n+1}, \mathbb{A}^{k-i+1}\right)\right)$. Formally, consider the following projection from the Stiefel manifold $S(n+1, k-i+1) \subset \operatorname{Hom}\left(\mathbb{A}^{n+1}, \mathbb{A}^{k-i+1}\right)$ of all homomorphisms of full rank onto the ambient Grassmannian of $\mathrm{CH}_{i}(X)$ :

$$
\begin{aligned}
p: \mathbb{P}(S(n+1, k-i+1)) & \longrightarrow \operatorname{Gr}\left(n-k+i-1, \mathbb{P}^{n}\right), \\
\varphi & \longmapsto \mathbb{P}(\operatorname{ker} \varphi),
\end{aligned}
$$

and take the closure $\overline{p^{-1}\left(\mathrm{CH}_{i}(X)\right)}$ in $\mathbb{P}\left(\operatorname{Hom}\left(\mathbb{A}^{n+1}, \mathbb{A}^{k-i+1}\right)\right)$.

Proposition 3.4. The varieties $\overline{p^{-1}\left(\mathrm{CH}_{i}(X)\right)}$ and $\left(X \times\left(\mathbb{P}^{k-i}\right)^{*}\right)^{\vee}$ are equal.

Proof. This proof follows the ideas of the proof of [43, Ch. 3, Thm. 2.7]. 
For $x \in \operatorname{Reg}(X)$ and $y \in\left(\mathbb{P}^{k-i}\right)^{*}$, we can compute the affine cone in $\mathcal{H}:=$ $\operatorname{Hom}\left(\mathbb{A}^{k-i+1}, \mathbb{A}^{n+1}\right)$ over the embedded tangent space of $X \times\left(\mathbb{P}^{k-i}\right)^{*}$ at $(x, y)$ as follows:

$$
\begin{aligned}
\mathbb{T}_{\boldsymbol{X} \times\left(\mathbf{P}^{\boldsymbol{k}-\boldsymbol{i}}\right)^{*},(\boldsymbol{x}, \boldsymbol{y})} & =\left(\mathbb{T}_{\boldsymbol{X}, \boldsymbol{x}} \otimes \boldsymbol{y}\right)+\left(\boldsymbol{x} \otimes\left(\mathbb{A}^{k-i+1}\right)^{*}\right) \\
& =\left\{\varphi \in \mathcal{H} \mid \operatorname{im} \varphi \subset \mathbb{T}_{\boldsymbol{X}, \boldsymbol{x}}, \boldsymbol{y}^{\vee} \subset \operatorname{ker} \varphi\right\}+\{\varphi \in \mathcal{H} \mid \operatorname{im} \varphi \subset \boldsymbol{x}\} \\
& =\left\{\varphi \in \mathcal{H} \mid \operatorname{im} \varphi \subset \mathbb{T}_{\boldsymbol{X}, \boldsymbol{x}}, \varphi\left(\boldsymbol{y}^{\vee}\right) \subset \boldsymbol{x}\right\} .
\end{aligned}
$$

The corresponding affine conormal space in $\mathcal{H}^{*}=\operatorname{Hom}\left(\mathbb{A}^{n+1}, \mathbb{A}^{k-i+1}\right)$ is

$$
\left(\mathcal{H} / \mathbb{T}_{\mathbf{X} \times\left(\mathbf{P}^{k-i}\right)^{*},(\boldsymbol{x}, \boldsymbol{y})}\right)^{*}=\left\{\psi \in \mathcal{H}^{*} \mid \psi\left(\mathbb{T}_{\mathbf{X}, \boldsymbol{x}}\right) \subset \boldsymbol{y}^{\vee}, \boldsymbol{x} \subset \operatorname{ker} \psi\right\} .
$$

Indeed, the linear spaces in (3.1) have the same dimension, and, for every $\varphi \in \mathbb{T}_{\mathbf{X \times}\left(\mathbf{P}^{\boldsymbol{k}-\boldsymbol{i}}\right)^{*},(\boldsymbol{x}, \boldsymbol{y})}$ and every $\psi \in \mathcal{H}^{*}$ contained in the right hand side of (3.1), we have $\operatorname{tr}(\psi \circ \varphi)=0$. Hence, we have computed $\left(X \times\left(\mathbb{P}^{k-i}\right)^{*}\right)^{\vee}$ explicitly:

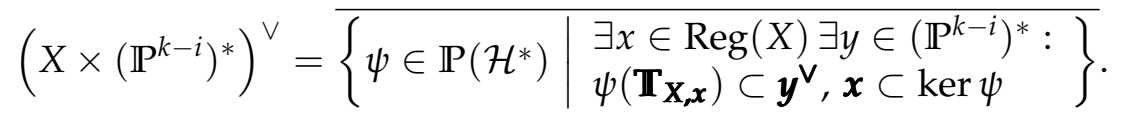

For $\psi \in \mathcal{H}^{*}$ and $x \in \operatorname{Reg}(X)$, note that $\psi\left(\mathbb{T}_{\boldsymbol{X}, \boldsymbol{x}}\right) \subset \boldsymbol{y}^{\vee}$ for some $y \in\left(\mathbb{P}^{k-i}\right)^{*}$ if and only if the restriction of $\psi$ to $\mathbb{T}_{\boldsymbol{X}, \boldsymbol{X}}$ is not surjective, which is equivalent to that the dimension of $\operatorname{ker}\left(\left.\psi\right|_{\mathbf{T}_{\mathbf{X}, \boldsymbol{x}}}\right)=\operatorname{ker} \psi \cap \mathbb{T}_{\boldsymbol{X}, \boldsymbol{x}}$ is at least $(k+1)-(k-i)=$ $i+1$. This shows

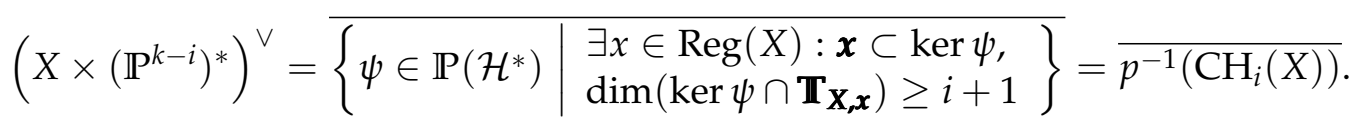

Proposition 3.4 shows that the defining polynomials of the coisotropic varieties (in case they are all hypersurfaces) interpolate from the Chow form via the Hurwitz form to the $X$-discriminant which is the defining polynomial of $X^{\vee}$. This raises immediately the next question: when is the $i$-th coisotropic variety indeed a hypersurface in its ambient Grassmannian.

Corollary 3.5. $\quad \mathrm{CH}_{i}(X)$ has codimension one in $\mathrm{Gr}\left(n-k+i-1, \mathbb{P}^{n}\right)$ if and only if $0 \leq i \leq k-\operatorname{codim} X^{\vee}+1$.

Proof. For any irreducible variety $X \subset \mathbb{P}^{n}$, let $\mu(X):=\operatorname{dim} X+\operatorname{codim} X^{\vee}-1$. As shown in [43, Ch. 1, Thm 5.5,], we have

$$
\mu(X \times Y)=\max \{\operatorname{dim} X+\operatorname{dim} Y, \mu(X), \mu(Y)\}
$$

for the product $X \times Y \hookrightarrow \mathbb{P}^{(n+1)(m+1)-1}$ of two irreducible varieties $X \subset \mathbb{P}^{n}$ and $Y \subset \mathbb{P}^{m}$. Hence, the dual $(X \times Y)^{\vee}$ is a hypersurface in $\left(\mathbb{P}^{(n+1)(m+1)-1}\right)^{*}$ if and only if the above maximum equals $\operatorname{dim} X+\operatorname{dim} Y$. This holds in particular when one of the factors is the variety $\mathbb{P}^{k-i}$ embedded into itself such 
that $\left(\mathbb{P}^{k-i}\right)^{\vee}=\varnothing\left[43\right.$, Ch. 1, Cor. 5.9]. By convention, $\operatorname{dim}\left(\mathbb{P}^{k-i}\right)^{\vee}=-1$, so $\mu\left(\mathbb{P}^{k-i}\right)=2(k-i)$. Thus $\left(X \times\left(\mathbb{P}^{k-i}\right)^{*}\right)^{\vee}$ is a hypersurface if and only if $2 k-i \geq k+\operatorname{codim} X^{\vee}-1$.

Example 3.6. Let $X:=Z\left(x_{0}^{3}+x_{1}^{3}+x_{2}^{3}+x_{3}^{3}\right) \subset \mathbb{P}^{3}$ be the Fermat cubic surface. The projectively dual of $X$ is also a surface. Therefore, the surface $X$ has three coisotropic hypersurfaces. The Chow form of $X$ in dual Plücker coordinates of $\operatorname{Gr}\left(0, \mathbb{P}^{3}\right)=\mathbb{P}^{3}$ is just the Fermat cubic itself. The Hurwitz form of $X$ in primal and dual Plücker coordinates of $\operatorname{Gr}\left(1, \mathbb{P}^{3}\right)$ is given by the polynomials in Example 2.3. This was computed with Macaulay2 (see CodeA in the Appendix). Finally, the second coisotropic form of $X$ in primal Plücker coordinates $p_{i}$ of $\operatorname{Gr}\left(2, \mathbb{P}^{3}\right)$, which are the dual coordinates of $\left(\mathbb{P}^{3}\right)^{*}$, is the following polynomial of degree 12 , which is also the defining equation of $X^{\vee}$ :

$$
\begin{aligned}
& 6\left(z_{0}^{4}+z_{1}^{4}+z_{2}^{4}+z_{3}^{4}\right)-8\left(z_{0}^{3}+z_{1}^{3}+z_{2}^{3}+z_{3}^{3}\right)\left(z_{0}+z_{1}+z_{2}+z_{3}\right) \\
+ & \left(z_{0}^{2}+z_{1}^{2}+z_{2}^{2}+z_{3}^{2}\right)^{2}+2\left(z_{0}^{2}+z_{1}^{2}+z_{2}^{2}+z_{3}^{2}\right)\left(z_{0}+z_{1}+z_{2}+z_{3}\right)^{2}-40 z_{0} z_{1} z_{2} z_{3},
\end{aligned}
$$

where $z_{i}:=p_{i}^{3}$ for $0 \leq i \leq 3$.

\subsection{Polar Degrees}

After studying the dimension of the coisotropic varieties in Definition 3.2, the next focus will lie on their degrees. In fact, these degrees agree with the wellstudied polar degrees, see [80] and [53]. As before, let $X \subset \mathbb{P}^{n}$ be an irreducible variety of dimension $k$. Moreover, let $0 \leq i \leq k$ and $V \subset \mathbb{P}^{n}$ be a projective subspace of dimension $n-k+i-2$. For almost all $x \in X$, the dimension of $V$ intersected with $\mathbb{T}_{X, x}$ equals $i-2$. We define the $i$-th polar variety of $X$ with respect to $V$ as

$$
P_{i}(X, V):=\overline{\left\{x \in \operatorname{Reg}(X) \mid \operatorname{dim}\left(V \cap \mathbb{T}_{X, x}\right) \geq i-1\right\}} \subset X .
$$

Given a general $X$, the $i$-th polar variety has codimension $i$ in $X$ for almost all choices of $V$. Furthermore, for any $X$ there exists an integer $\delta_{i}(X)$ that is equal to the degree of $P_{i}(X, V)$ for almost all $V$. This integer $\delta_{i}(X)$ is called the $i$-th polar degree of $X$.

These degrees satisfy a lot of interesting properties:

1. $\delta_{i}(X)>0$ if and only if $i \leq k-\operatorname{codim} X^{\vee}+1$.

(Note that this coincides with the range of indices where the coisotropic varieties of $X$ are hypersurfaces.)

2. $\delta_{0}(X)=\operatorname{deg} X$.

3. $\delta_{k-\operatorname{codim}} X^{\vee}+1(X)=\operatorname{deg} X^{\vee}$.

4. $\delta_{i}(X)=\delta_{k-\operatorname{codim}} X^{\vee}+1-i\left(X^{\vee}\right)$. 
5. $\delta_{i}(X \cap H)=\delta_{i}(X)$ for any $0 \leq i \leq k-1$ and any general hyperplane $H \subset \mathbb{P}^{n}$.

6. $\delta_{i}(\pi(X))=\delta_{i}(X)$ if codim $X \geq 2$ and $\pi: \mathbb{P}^{n} \rightarrow \mathbb{P}^{n-1}$ is a general linear projection.

One can also define the polar degrees via the conormal variety

$$
\mathcal{N}_{X, X^{\vee}}:=\overline{\left\{(x, y) \mid x \in \operatorname{Reg}(X), \mathbb{T}_{X, x} \subset y^{\vee}\right\}} \subset \mathbb{P}^{n} \times\left(\mathbb{P}^{n}\right)^{*}
$$

The multidegree of a variety $X$ embedded into a product of projective spaces $\mathbb{P}^{n_{1}} \times \ldots \times \mathbb{P}^{n_{d}}$ with codimension $c$ is a homogeneous polynomial of degree $c$ whose term $\alpha t_{1}^{c_{1}} \ldots t_{d}^{c_{d}}$ indicates that the intersection of $X$ with the product $L_{1} \times \ldots \times L_{d}$ of general projective subspaces $L_{i} \subset \mathbb{P}^{n_{i}}$ with $\operatorname{dim}\left(L_{i}\right)=c_{i}$ consists of $\alpha$ points. Thus, the multidegree of $\mathcal{N}_{X, X \vee}$ is a homogeneous polynomial of degree $n+1$ in two variables. The non-zero coefficients of this polynomial are the polar degrees (cf. [61, Prop. (3) on page 187] and [41, Lem. (2.23) on page 169]). Using the command multidegree in Macaulay2, this gives a practical way to compute the polar degrees of a given variety $X$.

Now another property will be added to the above list, namely that the degree of the $i$-th coisotropic hypersurface of $X$ is the $i$-th polar degree of $X$. On first sight, this may seem remarkable since the coisotropic hypersurfaces are subvarieties of a Grassmannian, whereas the polar varieties are subvarieties of the projective variety $X \subset \mathbb{P}^{n}$. The degree of a hypersurface $\Sigma \subset \operatorname{Gr}\left(\ell, \mathbb{P}^{n}\right)$ is defined as

$$
\operatorname{deg} \Sigma:=|\{L \in \Sigma \mid N \subset L \subset M\}|,
$$

where $N \subset M \subset \mathbb{P}^{n}$ is a general flag of $(\ell-1)$-dimensional and $(\ell+1)$ dimensional projective subspaces. Alternatively, the degree of $\Sigma$ can be defined as the degree of the defining polynomial of $\Sigma$ in the coordinate ring of $\operatorname{Gr}\left(\ell, \mathbb{P}^{n}\right)$ [43, Ch. 3, Prop. 2.1].

Example 3.7. The degree of the Chow hypersurface $\mathrm{CH}_{0}(X)$ in $\operatorname{Gr}\left(n-k-1, \mathbb{P}^{n}\right)$ of an irreducible variety $X \subset \mathbb{P}^{n}$ of dimension $k$ is the degree of $X$ : a general $(n-k)$-dimensional subspace $M$ intersects $X$ in exactly $\operatorname{deg}(X)$ points, so there are exactly $\operatorname{deg}(X)$ many $L \in \operatorname{Gr}\left(n-k-1, \mathbb{P}^{n}\right)$ that pass through a general $(n-k-2)$-dimensional subspace $N \subset M$ and intersect $X$; see Figure 3.1.

Theorem 3.8. For $0 \leq i \leq k-\operatorname{codim} X^{\vee}+1$, the degree of the $i$-th coisotropic hypersurface of $X$ equals the $i$-th polar degree of $X$, i.e.,

$$
\operatorname{deg} \mathrm{CH}_{i}(X)=\delta_{i}(X) \text {. }
$$

Proof. Let $0 \leq d \leq k$. For $0 \leq i \leq k-d$ and a general subspace $M \subset \mathbb{P}^{n}$ of codimension $d$, we have $\delta_{i}(X \cap M)=\delta_{i}(X)$ by applying the fifth property above several times. Fix now $0 \leq i \leq k-\operatorname{codim} X^{\vee}+1$ and set $d:=k-i$. Choose a general $M$ of codimension $d$ as well as a general subspace $N \subset M$ 


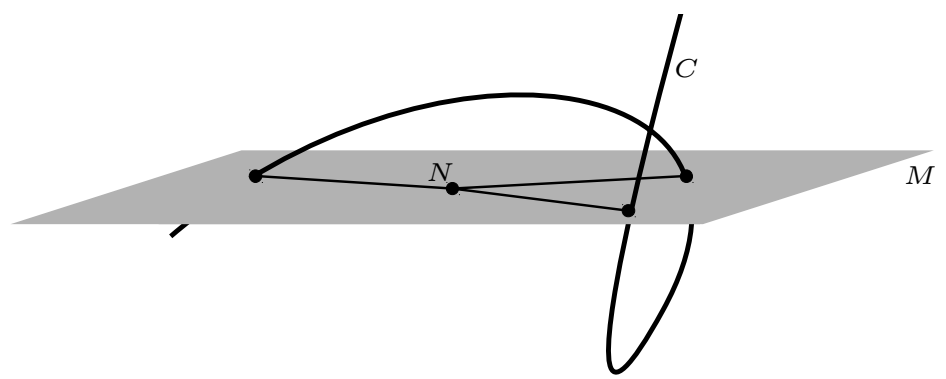

Figure 3.1: The degree of the Chow hypersurface of a space curve $C$ is $\operatorname{deg} C$.

with $\operatorname{dim} N=\operatorname{dim} M-\operatorname{dim}(X \cap M)+i-2=n-k+i-2$. Then the $i$-th polar degree of $X$ equals $\operatorname{deg} P_{i}(X \cap M, N)$. Since $X \cap M$ is $i$-dimensional, it follows from the first and the third property above that the dual $(X \cap M)^{\vee}$ is a hypersurface in $M^{*}$ with degree $\delta_{i}(X \cap M)$. To sum up,

$$
\delta_{i}(X)=\operatorname{deg} P_{i}(X \cap M, N)=\delta_{i}(X \cap M)=\operatorname{deg}(X \cap M)^{\vee} .
$$

The degree of the hypersurface $(X \cap M)^{\vee}$ is also the number of hyperplanes in $M$ that are tangent to $X \cap M$ at some smooth point and that contain $N$, but these hyperplanes are exactly the subspaces in $\mathrm{CH}_{i}(X)$ with $N \subset L \subset M$.

Remark 3.9. For general projective varieties $X$, we can give another geometric argument to show Theorem 3.8. As above, let $V$ be a general projective subspace of dimension $n-k+i-2$. Consider the variety $S_{i}(X, V) \subset \mathbb{P}^{n}$ formed by the union of all lines through $V$ and the $i$-th polar variety $P_{i}(X, V)$. For general $X$, the $i$-th polar variety has codimension $i$ in $X$ and $S_{i}(X, V)$ is a hypersurface of degree $\delta_{i}(X)$. The $i$-th coisotropic form of $X$ in dual Stiefel coordinates is a polynomial in the entries of a general $(n-k+i) \times(n+1)$-matrix $B$. Substituting the last rows of that matrix by a basis of $V$ yields a homogeneous polynomial $F \in \mathbb{C}\left[b_{0 j} \mid 0 \leq j \leq n\right]$, whose degree is the degree of the $i$-th coisotropic hypersurface of $X$. This polynomial defines an irreducible hypersurface in $\mathbb{P}^{n}$, which is in fact $S_{i}(X, V)$. This shows Theorem 3.8 for general $X$.

To see that $S_{i}(X, V)$ and the zero locus of $F$ are the same, it is enough to show that $F$ vanishes at every point of $S_{i}(X, V)$. This is clear for all points in $V \subset S_{i}(X, V)$. For a point $y \notin V$ on the line between $x \in P_{i}(X, V)$ and some point in $V$, we have that $F$ vanishes at $y$ if and only if it vanishes at $x$. If $x$ is a smooth point of $X$ such that the dimension of $V \cap \mathbb{T}_{X, x}$ is at least $i-1$, then the projective span of $V$ and $x$ is a point in $\mathrm{CH}_{i}(X)$ and $F$ vanishes at $x$. Since the set of all those $x$ is dense in $P_{i}(X, V)$, all points in $P_{i}(X, V)$ are in the zero locus of $F$. 


\subsection{Rank One Characterization}

The fundamental equivalence of the two notions of coisotropic hypersurfaces given in Definitions 3.1 and 3.2 was first proven in [43, Ch. 4, Thm. 3.14]. That proof contains many geometric ideas by taking a detour over conormal varieties and Lagrangian varieties in general. Here a new and direct proof will be presented. First, we compute the conormal spaces of the varieties $\mathrm{CH}_{i}(X)$ explicitly to see that they are coisotropic in the sense of Definition 3.1.

Proposition 3.10. Consider an irreducible variety $X$ in $\mathbb{P}^{n}$ and an integer $0 \leq i \leq \operatorname{dim} X-\operatorname{codim} X^{\vee}+1$. For $L \in \operatorname{Reg}\left(\mathrm{CH}_{i}(X)\right)$ such that there is exactly one $x \in \operatorname{Reg}(X) \cap L$ with $\operatorname{dim}\left(L \cap \mathbb{T}_{X, x}\right)=i$ we have that

$$
N_{\mathrm{CH}_{i}(X), L}^{*}=\left\{\varphi \in \operatorname{Hom}\left(\mathbb{A}^{n+1} / \boldsymbol{L}, \boldsymbol{L}\right) \mid \operatorname{im} \varphi \subset \boldsymbol{x},\left(\mathbb{T}_{\boldsymbol{X}, \boldsymbol{x}}+\boldsymbol{L}\right) / \boldsymbol{L} \subset \operatorname{ker} \varphi\right\} .
$$

In particular, $\mathrm{CH}_{i}(X)$ is coisotropic.

Proof. We denote by $\Sigma_{0}$ the set of all $L \in \operatorname{Reg}\left(\mathrm{CH}_{i}(X)\right)$ such that there is exactly one $x_{L} \in \operatorname{Reg}(X) \cap L$ with $\operatorname{dim}\left(L \cap \mathbb{T}_{X, x_{L}}\right)=i$. Note that $\Sigma_{0}$ is dense in $\mathrm{CH}_{i}(X)$. We consider the rational map

$$
\begin{aligned}
\omega: \mathrm{CH}_{i}(X) & \longrightarrow X, \\
\Sigma_{0} \ni L & \longmapsto x_{L}
\end{aligned}
$$

and a general $L \in \mathrm{CH}_{i}(X)$. By Corollary 2.9, we have $\left.\varphi\right|_{\boldsymbol{x}_{\boldsymbol{L}}}=\left(D_{L} \mathcal{\omega}(\varphi) \bmod L\right)$ for every $\varphi \in T_{\mathrm{CH}_{i}(X), L}$. Since the image of $D_{L} \mathcal{W}(\varphi)$ is contained in $\mathbb{T}_{\boldsymbol{X}, \boldsymbol{x}_{\mathbf{L}}} / \boldsymbol{x}_{\mathbf{L}}$, the tangent space $T_{\mathrm{CH}_{i}(X), L}$ is contained in $\left\{\varphi \in \operatorname{Hom}\left(\boldsymbol{L}, \mathbb{A}^{n+1} / \boldsymbol{L}\right) \mid \varphi\left(\boldsymbol{x}_{\boldsymbol{L}}\right) \subset\right.$ $\left.\left(\mathbb{T}_{\boldsymbol{X}, \boldsymbol{x}_{\boldsymbol{L}}}+\boldsymbol{L}\right) / \boldsymbol{L}\right\}$. This containment is actually an equality since the projective space $\mathbb{T}_{X, x_{L}}+L$ is a hyperplane in $\mathbb{P}^{n}$ due to $\operatorname{dim}\left(L \cap \mathbb{T}_{X, x_{L}}\right)=i$. Hence,

$$
\begin{aligned}
T_{\mathrm{CH}_{i}(X), L} & =\left\{\varphi \in \mathcal{H} \mid \varphi\left(\boldsymbol{x}_{\boldsymbol{L}}\right) \subset\left(\mathbb{T}_{\mathbf{X}, \boldsymbol{x}_{\boldsymbol{L}}}+\boldsymbol{L}\right) / \boldsymbol{L}\right\} \\
& =\left\{\varphi \in \mathcal{H} \mid \boldsymbol{x}_{\boldsymbol{L}} \subset \operatorname{ker} \varphi\right\}+\left\{\varphi \in \mathcal{H} \mid \operatorname{im} \varphi \subset\left(\mathbb{T}_{\boldsymbol{X}, \boldsymbol{x}_{\boldsymbol{L}}}+\boldsymbol{L}\right) / \boldsymbol{L}\right\},
\end{aligned}
$$

where $\mathcal{H}:=\operatorname{Hom}\left(\boldsymbol{L}, \mathbb{A}^{n+1} / \boldsymbol{L}\right)$. Since we have shown (3.3) for general $L$ in $\mathrm{CH}_{i}(X)$, it also holds for all $L \in \Sigma_{0}$. From this it follows immediately that $N_{\mathrm{CH}}^{*}(X), L$ is spanned by a rank one homomorphism with image $\boldsymbol{x}_{\boldsymbol{L}}$ and kernel $\left(\mathbb{T}_{X, x_{L}}+L\right) / L$.

Secondly, we need to construct for every coisotropic hypersurface (in the sense of Definition 3.1) an underlying projective variety. For points $x \in \mathbb{P}^{n}$ and $y \in\left(\mathbb{P}^{n}\right)^{*}$ such that $x \in y^{\vee}$, we define $\mathcal{G}_{\ell}(x, y):=\left\{L \in \operatorname{Gr}\left(\ell, \mathbb{P}^{n}\right) \mid x \in L \subset y^{\vee}\right\}$. Moreover, for an irreducible variety $X \subset \mathbb{P}^{n}$, we consider the following open subset of the conormal variety:

$$
\mathcal{U}_{X, X^{\vee}}:=\left\{(x, y) \in \operatorname{Reg}(X) \times \operatorname{Reg}\left(X^{\vee}\right) \mid \mathbb{T}_{X, x} \subset y^{\vee}\right\}
$$


Since the condition $\operatorname{dim}\left(L \cap \mathbb{T}_{X, x}\right) \geq i$ in the definition of $\mathrm{CH}_{i}(X)$ is equivalent to $\operatorname{dim}\left(L+\mathbb{T}_{X, x}\right) \leq n-1$, which means that $L$ is contained in a tangent hyperplane of $X$ at $x$, we have for $0 \leq i \leq \operatorname{dim} X$ that

$$
\mathrm{CH}_{i}(X)=\overline{\bigcup_{(x, y) \in \mathcal{U}_{X, X^{\vee}}} \mathcal{G}_{n-\operatorname{dim} X+i-1}(x, y)}
$$

Theorem 3.11. 1. For an irreducible variety $X$ in $\mathbb{P}^{n}$ and an integer $0 \leq i \leq \operatorname{dim} X-\operatorname{codim} X^{\vee}+1$, the $i$-th coisotropic hypersurface of $X$ is coisotropic (in the sense of Definition 3.1).

2. For an irreducible coisotropic hypersurface $\Sigma \subset \operatorname{Gr}\left(\ell, \mathbb{P}^{n}\right)$, there is an irreducible variety $X \subset \mathbb{P}^{n}$ such that $\Sigma=\mathrm{CH}_{\operatorname{dim} X+\ell+1-n}(X)$.

Proof. The first part was already proven in Proposition 3.10. For the second part, we consider the one-dimensional conormal space $N_{\Sigma, L}^{*} \subset \operatorname{Hom}\left(\mathbb{A}^{n+1} / L, L\right)$ at $L \in \operatorname{Reg}(\Sigma)$. It is spanned by a rank one homomorphism. Hence, there are unique $x_{L} \in L$ and $y_{L} \in\left(\mathbb{P}^{n}\right)^{*}$ such that $L \subset y_{L^{\prime}}^{\vee}$

$$
\begin{aligned}
& N_{\Sigma, L}^{*}=\left\{\varphi \in \operatorname{Hom}\left(\mathbb{A}^{n+1} / \boldsymbol{L}, \boldsymbol{L}\right) \mid \operatorname{im} \varphi \subset \boldsymbol{x}_{\boldsymbol{L}}, \boldsymbol{y}_{\boldsymbol{L}}^{\vee} / \boldsymbol{L} \subset \operatorname{ker} \varphi\right\}, \text { and } \\
& T_{\Sigma, L}=\left\{\varphi \in \operatorname{Hom}\left(\boldsymbol{L}, \mathbb{A}^{n+1} / \boldsymbol{L}\right) \mid \varphi\left(\boldsymbol{x}_{\boldsymbol{L}}\right) \subset \boldsymbol{y}_{\boldsymbol{L}}^{\vee} / \boldsymbol{L}\right\} .
\end{aligned}
$$

Thus, we can define the rational map $\omega: \Sigma \rightarrow \mathbb{P}^{n}$ which maps $L \in \operatorname{Reg}(\Sigma)$ to $x_{L}$. We denote the Zariski closure of the image of $\omega$ by $X \subset \mathbb{P}^{n}$. Since $\Sigma$ is irreducible, so is $X$. By Corollary 2.9, the differential of $\omega$ at a general $L \in \Sigma$ is a surjection $\omega_{L}: T_{\Sigma, L} \rightarrow T_{X, x_{L}}$ such that $\left.\varphi\right|_{x_{\boldsymbol{L}}}=\left(\omega_{L}(\varphi) \bmod \boldsymbol{L}\right)$ for every $\varphi \in T_{\Sigma, L}$. Due to (3.7), we see that the image of each $\psi \in T_{X, x_{L}}$ is contained in $\boldsymbol{y}_{\boldsymbol{L}}^{\vee} / \boldsymbol{x}_{\mathbf{L}}$, which means that $\mathbb{T}_{X, x_{L}} \subset y_{L}^{\vee}$. Hence, using (3.5), we have shown that $\Sigma \subset \mathrm{CH}_{i}(X)$, where $i:=\operatorname{dim} X+\ell+1-n$. Since $\Sigma$ is a hypersurface and $\mathrm{CH}_{i}(X) \subset \operatorname{Gr}\left(\ell, \mathbb{P}^{n}\right)$ is a proper irreducible subvariety, we have $\Sigma=\mathrm{CH}_{i}(X)$.

Example 3.12. All coisotropic hypersurfaces in the Grassmannian $\operatorname{Gr}\left(1, \mathbb{P}^{3}\right)$ of lines in $\mathbb{P}^{3}$ are either Chow hypersurfaces of curves or Hurwitz hypersurfaces of surfaces. According to [16], these define exactly the self-dual hypersurfaces in $\operatorname{Gr}\left(1, \mathbb{P}^{3}\right)$.

\subsection{Duality}

By Remark/Definition 2.6, a hypersurface $\Sigma \subset \operatorname{Gr}\left(\ell, \mathbb{P}^{n}\right)$ is coisotropic if and only if $\Sigma^{\perp} \subset \operatorname{Gr}\left(n-\ell-1,\left(\mathbb{P}^{n}\right)^{*}\right)$ is coisotropic. In that case, their underlying projective varieties are projectively dual to each other. 
Theorem 3.13. For an irreducible variety $X$ in $\mathbb{P}^{n}$ as well as an integer $0 \leq i \leq \operatorname{dim} X-\operatorname{codim} X^{\vee}+1$, we have

$$
\mathrm{CH}_{i}(X)^{\perp}=\mathrm{CH}_{\operatorname{dim} X-\operatorname{codim} X^{\vee}+1-i}\left(X^{\vee}\right) .
$$

Proof. We set $\ell:=n-\operatorname{dim} X+i-1$ and use (3.5). First, we clearly have $\mathcal{G}_{\ell}(x, y)^{\perp}=\mathcal{G}_{n-\ell-1}(y, x)$. Secondly, the biduality theorem implies the equality $\mathcal{U}_{X^{\vee}, X}=\left\{(y, x) \mid(x, y) \in \mathcal{U}_{X, X^{\vee}}\right\}$ (see (3.4)). Hence,

$$
\begin{aligned}
\mathrm{CH}_{i}(X)^{\perp} & =\overline{\bigcup_{(x, y) \in \mathcal{U}_{X, X \vee}} \mathcal{G}_{\ell}(x, y)^{\perp}} \\
& =\frac{\bigcup_{(y, x) \in \mathcal{U}_{X \vee}, X} \mathcal{G}_{n-\ell-1}(y, x)}{\bigcup^{\prime}}=\mathrm{CH}_{\operatorname{dim} X-\operatorname{codim} X^{\vee}+1-i}\left(X^{\vee}\right) .
\end{aligned}
$$

Note that Theorems 3.8 and 3.13 give an alternative proof that the polar degrees of a projective variety and its dual are the same but in reversed order, as stated in the forth property of polar degrees in Section 3.2

Corollary 3.14 (Dual Cayley Trick). Using the projection

$$
\begin{aligned}
q: \mathbb{P}(S(n-\operatorname{dim} X+i, n+1)) & \longrightarrow \operatorname{Gr}\left(n-\operatorname{dim} X+i-1, \mathbb{P}^{n}\right), \\
\varphi & \longmapsto \mathbb{P}(\operatorname{im} \varphi)
\end{aligned}
$$

sending full rank homomorphisms in $\mathbb{P}\left(\operatorname{Hom}\left(\mathbb{A}^{n-\operatorname{dim} X+i}, \mathbb{A}^{n+1}\right)\right)$ to their projectivized image, we have

$$
\overline{q^{-1}\left(\mathrm{CH}_{i}(X)\right)}=\left(\mathbb{P}^{n-\operatorname{dim} X+i-1} \times X^{\vee}\right)^{\vee} .
$$

Proof. We set $k:=\operatorname{dim} X$ and consider the Segre embedding

$$
\begin{array}{r}
\mathbb{P}^{n-k+i-1} \times X^{\vee} \hookrightarrow \mathbb{P}^{n-k+i-1} \times\left(\mathbb{P}^{n}\right)^{*} \hookrightarrow \mathbb{P}\left(\operatorname{Hom}\left(\mathbb{A}^{n+1}, \mathbb{A}^{n-k+i}\right)\right) \\
\text { and }\left(\mathbb{P}^{n-k+i-1} \times X^{\vee}\right)^{\vee} \hookrightarrow \mathbb{P}\left(\operatorname{Hom}\left(\mathbb{A}^{n-k+i}, \mathbb{A}^{n+1}\right)\right) .
\end{array}
$$

Moreover, we define

$$
\begin{aligned}
p: \mathbb{P}(S(n-k+i, n+1)) & \longrightarrow \operatorname{Gr}\left(k-i,\left(\mathbb{P}^{n}\right)^{*}\right), \\
\varphi & \longmapsto \mathbb{P}\left(\operatorname{ker}\left(\varphi^{*}\right)\right) .
\end{aligned}
$$

In particular, we have $q(\varphi)^{\vee}=p(\varphi)$. This yields together with Proposition 3.4 and Theorem 3.13 for $j:=k-\operatorname{codim} X^{\vee}+1-i$ that

$$
\overline{q^{-1}\left(\mathrm{CH}_{i}(X)\right)}=\overline{q^{-1}\left(\mathrm{CH}_{j}\left(X^{\vee}\right)^{\perp}\right)}=\overline{p^{-1}\left(\mathrm{CH}_{j}\left(X^{\vee}\right)\right)}=\left(\mathbb{P}^{\operatorname{dim} X^{\vee}-j} \times X^{\vee}\right)^{\vee} .
$$


Note that the two factors of the Segre product appear in a different order than in Proposition 3.4 since the map $\varphi \mapsto \varphi^{*}$ exchanges the factors of the tensor product as in (2.3).

Example 3.15. The Chow form of a curve of degree at least two in $\mathbb{P}^{3}$ is naturally isomorphic to the Hurwitz form of its dual surface. Thus, a line intersects the curve if and only if its dual line is tangent to the dual surface. For example, the dual of the twisted cubic is the surface cut out by the discriminant of a cubic univariate polynomial. By Example 1.1. the Chow form of the twisted cubic in primal Plücker coordinates on $\operatorname{Gr}\left(1, \mathbb{P}^{3}\right)$ is the determinant of the Bézout matrix $B$ :

$$
B:=\left[\begin{array}{ccc}
p_{01} & p_{02} & p_{03} \\
p_{02} & p_{03}+p_{12} & p_{13} \\
p_{03} & p_{13} & p_{23}
\end{array}\right] \leadsto\left[\begin{array}{ccc}
q_{23} & -q_{13} & q_{12} \\
-q_{13} & q_{03}+q_{12} & -q_{02} \\
q_{12} & -q_{02} & q_{01}
\end{array}\right],
$$

and the Hurwitz form of the discriminant surface in primal Plücker coordinates on $\operatorname{Gr}\left(1,\left(\mathbb{P}^{3}\right)^{*}\right)$ is the determinant of the matrix on the right.

Example 3.16. The Hurwitz form of a general surface in $\mathbb{P}^{3}$ is naturally isomorphic to the Hurwitz form of its dual surface. Consider for example the selfdual Segre surface $\mathbb{P}^{1} \times \mathbb{P}^{1}$. Its Hurwitz form is the determinant of the matrix $\left[\begin{array}{cc}2 p_{02} & p_{12}+p_{03} \\ p_{12}+p_{03} & 2 p_{13}\end{array}\right]$ (see Code $\mathrm{B}$ in the Appendix), which stays invariant under the change of coordinates (2.4).

This phenomenon can also be observed in Example 3.6. The hypersurface in $\operatorname{Gr}\left(1,\left(\mathbb{P}^{3}\right)^{*}\right)$ whose defining equation in primal Plücker coordinates is $(2.6)$ and whose defining equation in dual Plücker coordinates is $(2.5)$ is the Hurwitz hypersurface of the projectively dual $X^{\vee}$ of the Fermat cubic surface $X \subset \mathbb{P}^{3}$. Hence, the two similar polynomials of degree six in Example 2.3 are the Hurwitz forms of the surface $X$ of degree three and the surface $X^{\vee}$ of degree twelve. Moreover, the second coisotropic hypersurface of $X^{\vee}$ is the Fermat cubic surface $X$.

Example 3.17. In $\operatorname{Gr}\left(2, \mathbb{P}^{4}\right)$, there are three cases for coisotropic hypersurfaces: Chow forms of curves, Hurwitz forms of surfaces, and second coisotropic forms of threefolds. On the other hand, there are just two cases for $\operatorname{Gr}\left(1, \mathbb{P}^{4}\right)$, namely Chow forms of surfaces and Hurwitz forms of threefolds. The following table summarizes which forms coincide, depending on the dimensions of the variety $X$ and its dual $X^{\vee}$.

\begin{tabular}{r|c|c|c}
$X \mid X^{\vee}$ & curve & surface & threefold \\
\hline curve & & $\mathrm{CH}_{0}(X)^{\perp}=\mathrm{CH}_{0}\left(X^{\vee}\right)$ & $\mathrm{CH}_{0}(X)^{\perp}=\mathrm{CH}_{1}\left(X^{\vee}\right)$ \\
\hline surface & $\mathrm{CH}_{0}(X)^{\perp}=\mathrm{CH}_{0}\left(X^{\vee}\right)$ & $\mathrm{CH}_{0}(X)^{\perp}=\mathrm{CH}_{1}\left(X^{\vee}\right)$ & $\mathrm{CH}_{0}(X)^{\perp}=\mathrm{CH}_{2}\left(X^{\vee}\right)$ \\
& & $\mathrm{CH}_{1}(X)^{\perp}=\mathrm{CH}_{0}\left(X^{\vee}\right)$ & $\mathrm{CH}_{1}(X)^{\perp}=\mathrm{CH}_{1}\left(X^{\vee}\right)$ \\
\hline threefold & $\mathrm{CH}_{1}(X)^{\perp}=\mathrm{CH}_{0}\left(X^{\vee}\right)$ & $\mathrm{CH}_{1}(X)^{\perp}=\mathrm{CH}_{1}\left(X^{\vee}\right)$ & $\mathrm{CH}_{1}(X)^{\perp}=\mathrm{CH}_{2}\left(X^{\vee}\right)$ \\
& & $\mathrm{CH}_{2}(X)^{\perp}=\mathrm{CH}_{0}\left(X^{\vee}\right)$ & $\mathrm{CH}_{2}(X)^{\perp}=\mathrm{CH}_{1}\left(X^{\vee}\right) \diamond$
\end{tabular}




\subsection{Hyperdeterminants}

The purpose of this section is to derive and discuss the following result.

Proposition 3.18. The $i$-th coisotropic form of the Segre variety $\mathbb{P}^{n_{1}} \times \ldots \times \mathbb{P}^{n_{d}}$ in $\mathbb{P}^{\left(n_{1}+1\right) \cdots\left(n_{d}+1\right)-1}$, in primal Stiefel coordinates, coincides with the hyperdeterminant of format $\left(n_{1}+1\right) \times \ldots \times\left(n_{d}+1\right) \times\left(n_{1}+\ldots+n_{d}-i+1\right)$. All hyperdeterminants arise in that manner.

Chapter 14 of [43] is devoted to the study of hyperdeterminants. They are defined as follows. For $n_{1}, \ldots, n_{d} \geq 1$, the variety $X:=\mathbb{P}^{n_{1}} \times \ldots \times \mathbb{P}^{n_{d}}$ in $\mathbb{P}^{\left(n_{1}+1\right) \cdots\left(n_{d}+1\right)-1}$ characterizes all tensors of format $\left(n_{1}+1\right) \times \ldots \times\left(n_{d}+1\right)$ having rank at most one. Whenever the dual variety $X^{\vee}$ is a hypersurface, its defining polynomial is called the hyperdeterminant of format $\left(n_{1}+1\right) \times \ldots \times\left(n_{d}+1\right)$. Analogously to Corollary 3.5, one can derive the condition for $\operatorname{codim} X^{\vee}=1$ : recall that $\mu(Y):=\operatorname{dim} Y+\operatorname{codim} Y^{\vee}-1$ for every irreducible variety $Y \subset \mathbb{P}^{n}$. The equality (3.2) proven in [43, Ch. 1, Thm. 5.5] generalizes by induction to

$$
\mu\left(X_{1} \times \ldots \times X_{d}\right)=\max \left\{\operatorname{dim} X_{1}+\ldots+\operatorname{dim} X_{d}, \mu\left(X_{1}\right), \ldots, \mu\left(X_{d}\right)\right\} .
$$

Hence, $X^{\vee}$ is a hypersurface if and only if $2 n_{i} \leq n_{1}+\ldots+n_{d}$ for all $i=1, \ldots, d$. More generally,

$$
\operatorname{codim} X^{\vee}=\max \left\{1,2 \max \left\{n_{1}, \ldots, n_{d}\right\}-\left(n_{1}+\ldots+n_{d}\right)+1\right\} .
$$

Example 3.19. In the special case $d=2$ of matrices, the projectively dual variety $X^{\vee}$ is given by all matrices that do not have full rank. This variety is a hypersurface if and only if the matrices have square format $\left(n_{1}=n_{2}\right)$. In this case, the defining polynomial of $X^{\vee}$ is the usual determinant. Otherwise the codimension of $X^{\vee}$ equals $\left|n_{2}-n_{1}\right|+1$.

Proof of Proposition 3.18. Let $0 \leq i \leq n_{1}+\ldots+n_{d}-\operatorname{codim} X^{\vee}+1$. By the Cayley trick in Proposition 3.4 , the $i$-th coisotropic form of $X$ written in primal Stiefel coordinates is exactly the hyperdeterminant of format $\left(n_{1}+1\right) \times \ldots \times$ $\left(n_{d}+1\right) \times\left(n_{1}+\ldots+n_{d}-i+1\right)$. It is clear that all hyperdeterminants arise in that way as coisotropic forms of the varieties of tensors with rank at most one.

Remark 3.20. Note that even the usual determinant of square matrices is given by the Chow form of $\mathbb{P}^{n}$. Using the duality explained in Theorem 3.13, the hyperdeterminants can also be characterized as the coisotropic forms of the varieties of degenerate tensors.

If all inequalities $2 n_{i} \leq n_{1}+\ldots+n_{d}$ are satisfied (which means that $X^{\vee}$ is a hypersurface) such that at least one of them holds with equality, the hyperdeterminant is said to be of boundary format. An example for this is the determinant of square matrices. The hyperdeterminants of boundary format can also be 
characterized in terms of coisotropic forms. This is also studied in [43, Ch. 14, Sec. 3C], but here this naturally and immediately follows from the duality studied in Theorem 3.13 .

Corollary 3.21. The Chow form of the Segre variety $X=\mathbb{P}^{n_{1}} \times \ldots \times \mathbb{P}^{n_{d}}$ in primal Stiefel coordinates is a hyperdeterminant of boundary format, and - up to permuting the tensor format - all hyperdeterminants of boundary format arise in that manner.

If codim $X^{\vee} \geq 2$, then the Chow form of $X^{\vee}$ in dual Stiefel coordinates is a hyperdeterminant of boundary format, and - up to permuting the tensor format - all hyperdeterminants of boundary format arise in that manner.

Proof. The first part of this proposition is clear. Note that the second part uses the convention that the Chow form of the empty variety $\left(\mathbb{P}^{n}\right)^{\vee}$ in dual Stiefel coordinates is the usual $(n+1) \times(n+1)$-determinant. If $X^{\vee}$ is not a hypersurface and $d \geq 2$, exactly two coisotropic forms of $X$ yield hyperdeterminants of boundary format: its Chow form and its $\left(2 \cdot\left(n_{1}+\ldots+n_{d}-\max \left\{n_{1}, \ldots, n_{d}\right\}\right)\right)$ th coisotropic form, where - by Theorem 3.13-the latter is naturally isomorphic to the Chow form of $X^{\vee}$. So, although $X^{\vee}$ is not defining a hyperdeterminant, its Chow form in dual Stiefel coordinates is the hyperdeterminant of boundary format $\left(n_{1}+1\right) \times \ldots \times\left(n_{d}+1\right) \times\left(2 \max \left\{n_{1}, \ldots, n_{d}\right\}-\left(n_{1}+\ldots+n_{d}\right)+1\right)$.

Remark 3.22. Theorem 3.3 in [43, Ch. 14] shows that all hyperdeterminants of boundary format can be written as the usual determinant of a square matrix whose entries are linear forms in the tensor entries. Hence, if $X^{\vee}$ is not a hypersurface, the Chow forms of $X$ and $X^{\vee}$ have determinantal representations in their Stiefel coordinates.

Analogously, if $X^{\vee}$ is a hypersurface and the corresponding hyperdeterminant is of boundary format, the Chow form of $X$ and the $\left(n_{1}+\ldots+n_{d}\right)$-th coisotropic hypersurface of $X$ (which is just $X^{\vee}$ ) give hyperdeterminants of boundary format. These are the only two coisotropic hypersurfaces of $X$ with that property, and their defining polynomials in Stiefel coordinates have determinantal representations. Finally, if $X^{\vee}$ is a hypersurface and its hyperdeterminant is not of boundary format, the Chow form of $X$ is the only coisotropic form which yields a hyperdeterminant of boundary format. In all cases the Chow form of $X$ has a determinantal representation in primal Stiefel coordinates.

Example 3.23. The variety $X=\mathbb{P}^{1} \times \mathbb{P}^{n}$ of $2 \times(n+1)$-matrices of rank at most one is self-dual and it has three coisotropic hypersurfaces. Hence, after the change of coordinates in (2.4), the Chow form of $X$ is the same as the second coisotropic form of $X$.

For $n=1$, the variety $X$ itself is a hypersurface, given by the $2 \times 2$-determinant. Therefore, its Chow form and its second coisotropic form are also the $2 \times 2$-determinant in their respective Plücker coordinates. As mentioned in Example 3.16, the Hurwitz form of $X$ is the determinant of $\left[\begin{array}{cc}2 p_{02} & p_{12}+p_{03} \\ p_{12}+p_{03} & 2 p_{13}\end{array}\right]$, which leads after substitution by the $2 \times 2$-minors of a general $2 \times 4$-matrix to the hyperdeterminant of format $2 \times 2 \times 2$. 
Analogously, the Chow form of $X$ in primal Stiefel coordinates is the hyperdeterminant of boundary format $2 \times 2 \times 3$. Hence, this hyperdeterminant can be written as the determinant of $\left[\begin{array}{lll}p_{012} & p_{013} \\ p_{023} & p_{123}\end{array}\right]$, where the $p_{i j k}$ are the $3 \times 3$-minors of a general $3 \times 4$-matrix. On the other hand, the $2 \times 2 \times 3$-hyperdeterminant has a determinantal representation: let $A, B \in \mathbb{A}^{2 \times 3}$ be the two slices of a general $2 \times 2 \times 3$-tensor in the first direction. The $2 \times 2 \times 3$-hyperdeterminant is the determinant of the $6 \times 6$-matrix $\left[\begin{array}{ccc}A^{T} & B^{T} & 0 \\ 0 & A^{T} & B^{T}\end{array}\right]$, since by Laplace expansion in the first three rows this determinant is equal to $p_{012} p_{123}-p_{013} p_{023}$, where the $p_{i j k}$ are the minors of the $3 \times 4$-matrix $\left[A^{T} \mid B^{T}\right]$. Moreover, the $2 \times 3 \times 2$-hyperdeterminant is also given by the second coisotropic form of $\mathbb{P}^{1} \times \mathbb{P}^{2}$ in primal Stiefel coordinates, or equivalently by the Chow form of $\mathbb{P}^{1} \times \mathbb{P}^{2}$ in dual Stiefel coordinates. Thus, the $2 \times 3 \times 2$-hyperdeterminant can be obtained by substituting the $2 \times 2$-minors of the general $2 \times 6$-matrix $[A \mid B]$ into the following Chow form of $\mathbb{P}^{1} \times \mathbb{P}^{2}$ (see Code $\mathrm{C}$ in the Appendix):

$$
\begin{aligned}
& q_{12} q_{23} q_{34}-q_{02} q_{24} q_{34}-q_{12} q_{13} q_{35}+q_{02} q_{14} q_{35}-q_{01} q_{15} q_{35} \\
+ & q_{01} q_{24} q_{35}-q_{02} q_{04} q_{45}+q_{01} q_{05} q_{45}+q_{02} q_{13} q_{45}-2 q_{01} q_{23} q_{45}
\end{aligned}
$$

\subsection{Coordinate Systems of Grassmannians}

For explicit computations it is important to be able to check for coisotropy in different coordinate system of Grassmannians. We will investigate affine, Stiefel and Plücker coordinates in this section. Due to the duality explained in Section 3.4, it is enough to consider dual coordinates only. All statements presented here will hold analogously for primal coordinates.

We begin with dual Stiefel coordinates. For this, we consider the rational map $\rho$ in (2.10), which is defined on the set of full rank matrices, and the Plücker embedding $\mathrm{pl}$ in (2.11). So the map $\mu:=\mathrm{pl} \circ \rho$ sends a matrix of full rank to its maximal minors; see the diagram before Proposition 3.25. If $Q$ denotes the defining equation of a hypersurface $\Sigma \subset \operatorname{Gr}\left(\ell+1, \mathbb{A}^{n+1}\right)$ in dual Plücker coordinates, then $Q \circ \mu$ denotes the defining equation of the hypersurface $\overline{\rho^{-1}(\Sigma)}$ in $\mathbb{A}^{(\ell+1) \times(n+1)}$. Note that $\overline{\rho^{-1}(\Sigma)}$ is an affine cone and that the projectively dual of $Y:=\mathbb{P}\left(\overline{\rho^{-1}(\Sigma)}\right)$ is the Zariski closure of the image of

$$
\begin{aligned}
& Y \longrightarrow \mathbb{P}\left(\mathbb{A}^{(\ell+1) \times(n+1)}\right)^{*}, \\
& A \longmapsto J_{Q \circ \mu}(A),
\end{aligned}
$$

where $J_{Q \circ \mu}$ is the $(\ell+1) \times(n+1)$-matrix of partial derivatives of $Q \circ \mu$.

Proposition 3.24. Let $\Sigma \subset \operatorname{Gr}\left(\ell+1, \mathbb{A}^{n+1}\right)$ be an irreducible hypersurface, given by a homogeneous polynomial $Q$ in dual Plücker coordinates. Moreover, we consider $Y:=\mathbb{P}\left(\overline{\rho^{-1}(\Sigma)}\right)=Z(Q \circ \mu) \subset \mathbb{P}\left(\mathbb{A}^{(\ell+1) \times(n+1)}\right)$. The following are equivalent:

1. $\Sigma$ is coisotropic. 
2. The Segre variety $\left(\mathbb{P}^{\ell}\right)^{*} \times\left(\mathbb{P}^{n}\right)^{*} \hookrightarrow \mathbb{P}\left(\mathbb{A}^{(\ell+1) \times(n+1)}\right)^{*}$ contains $Y^{\vee}$.

3. The rank of $J_{Q \circ \mu}(A)$ is at most one for all $A \in Y$.

Proof. Clearly, the second and the third assertion are equivalent. To show the equivalence with assertion one, we first assume that $\Sigma$ is coisotropic. By Theorem 3.11 and the dual Cayley trick in Corollary 3.14, we have that $\Sigma$ is a coisotropic hypersurface of some variety $X \subset \mathbb{P}^{n}$ such that $Y^{\vee}$ is equal to the Segre product $\left(\mathbb{P}^{\ell}\right)^{*} \times X^{\vee}$. This shows that assertions two and three hold if $\Sigma$ is coisotropic.

For the other direction, let $L \in \operatorname{Reg}(\Sigma)$ and $A \in \rho^{-1}(L) \cap \operatorname{Reg}(\boldsymbol{Y})$. We denote the rows of a matrix $M \in \mathbb{A}^{(\ell+1) \times(n+1)}$ by $M_{0}, \ldots, M_{\ell}$. Assertions two and three imply that $T_{\boldsymbol{Y}, A}$ is the zero locus of $\sum_{i, j} \lambda_{i} z_{j} x_{i j}$, where $\lambda_{0}, \ldots, \lambda_{\ell}$ and $z_{0}, \ldots, z_{n}$ are constants. From

$$
\left\{M \in \mathbb{A}^{(\ell+1) \times(n+1)} \mid \forall i=0, \ldots, \ell: M_{i} \in L\right\}=T_{\rho^{-1}(L), A} \subset T_{\boldsymbol{Y}, A}=Z\left(\sum_{i, j} \lambda_{i} z_{j} x_{i j}\right)
$$

it follows that $L$ is contained in the hyperplane $H:=Z\left(\sum_{j=0}^{n} z_{j} x_{j}\right) \subset \mathbb{A}^{n+1}$. By Corollary 2.10, the image of $T_{Y, A}$ under the differential of $\rho$ at $A$ consists of all $\varphi \in \operatorname{Hom}\left(L, \mathbb{A}^{n+1} / L\right)$ with $\varphi\left(\sum_{i=0}^{\ell} \lambda_{i} A_{i}\right) \in H / L$. Since $\Sigma$ is a hypersurface and $p:=\sum_{i=0}^{\ell} \lambda_{i} A_{i} \neq 0$, we have $T_{\Sigma, L}=\left\{\varphi \in \operatorname{Hom}\left(L, \mathbb{A}^{n+1} / L\right) \mid \varphi(p) \in H / L\right\}$. This shows that $N_{\Sigma, L}^{*} \subset \operatorname{Hom}\left(\mathbb{A}^{n+1} / L, L\right)$ is spanned by a rank one homomorphism with kernel $H / L$ and image $\operatorname{span}\{p\}$. Thus, $\Sigma$ is coisotropic.

Proposition 3.24 provides a practical tool to check if a given hypersurface of a Grassmannian is coisotropic. It might be more efficient to run this computation in an affine chart of the Grassmannian. This motivates the following proposition, which asserts that it is enough to check for coisotropy of a hypersurface of a Grassmannian in one fixed affine chart to deduce coisotropy for the whole hypersurface. This statement can also be found as Proposition 3.12 in [43, Ch. 4].

The set up is the following. We fix integers $0 \leq j_{0}<\ldots<j_{\ell} \leq n$ and consider the map $\iota:=\iota_{j_{0} \ldots j_{\ell}}$ which sends a matrix $A \in \mathbb{A}^{(\ell+1) \times(n-\ell)}$ to the matrix $\iota(A) \in$ $\mathbb{A}^{(\ell+1) \times(n+1)}$ whose columns indexed by $j_{0}, \ldots, j_{\ell}$ form the identity matrix and whose remaining columns form $A$. Thus, if $Q$ is the defining polynomial in dual Plücker coordinates of a hypersurface $\Sigma \subset \operatorname{Gr}\left(\ell+1, \mathbb{A}^{n+1}\right)$, then $Q \circ \mu \circ \iota$ is the equation for $\Sigma$ in the dual affine chart $\left\{L \in \operatorname{Gr}\left(\ell+1, \mathbb{A}^{n+1}\right) \mid q_{j_{0} \ldots j_{\ell}}(L) \neq 0\right\} \cong$ $\mathbb{A}^{(\ell+1) \times(n-\ell)}$. We summarize the used maps in the following commutative diagram:

$$
\begin{aligned}
\mathbb{A}^{(\ell+1) \times(n-\ell)} \stackrel{\iota}{\longrightarrow} & \mathbb{A}^{(\ell+1) \times(n+1)} \\
& \\
& \operatorname{Gr}\left(\ell+1, \mathbb{A}^{n+1}\right) \stackrel{\mathrm{pl}}{\longrightarrow} \mathbb{P}\left(\Lambda^{\ell+1} \mathbb{A}^{n+1}\right)
\end{aligned}
$$


Proposition 3.25. Let $\Sigma \subset \operatorname{Gr}\left(\ell+1, \mathbb{A}^{n+1}\right)$ be an irreducible hypersurface, given by a homogeneous polynomial $Q$ in dual Plücker coordinates. Moreover, fix a dual affine chart $\left\{L \mid q_{j_{0} \ldots j_{\ell}}(L) \neq 0\right\} \subset \operatorname{Gr}\left(\ell+1, \mathbb{A}^{n+1}\right)$ together with the map $\iota:=\iota_{j_{0} \ldots, j_{\ell}}$ and let $Z:=(\rho \circ \iota)^{-1}(\Sigma)=Z(Q \circ \mu \circ \iota) \subset \mathbb{A}^{(\ell+1) \times(n-\ell)}$. If $Z \neq \varnothing$, then $\Sigma$ is coisotropic if and only if the rank of the $(\ell+1) \times(n-\ell)$-matrix $J_{Q \circ \mu \circ \iota}(A)$ of partial derivatives of $Q \circ \mu \circ \iota$ is at most one for all $A \in Z$.

Proof. We may assume that $\left(j_{0}, \ldots, j_{\ell}\right)=(n-\ell, \ldots, n)$. Defining $Y$ as in Proposition 3.24 and denoting by $I_{\ell+1}$ the $(\ell+1) \times(\ell+1)$-identity matrix, we have for a general $A \in Z$ that

$$
\begin{aligned}
\left.\left(D_{A} l\right)\right|_{T_{Z, A}}: T_{Z, A} & \longrightarrow T_{\boldsymbol{Y},\left[A \mid I_{\ell+1}\right]}, \\
M & \longmapsto[M \mid 0] .
\end{aligned}
$$

We need to show that $\Sigma$ is coisotropic if and only if, for a general $A \in Z$, there are $\lambda \in \mathbb{A}^{\ell+1}$ and $z \in \mathbb{A}^{n-\ell}$ such that

$$
T_{Z, A}=Z\left(\sum_{i=0}^{\ell} \sum_{j=0}^{n-\ell-1} \lambda_{i} z_{j} x_{i j}\right)
$$

First, we assume that the hypersurface $\Sigma$ is coisotropic. By Proposition 3.24 . there are $\lambda \in \mathbb{A}^{\ell+1}$ and $z^{\prime} \in \mathbb{A}^{n+1}$ such that $T_{\boldsymbol{Y},\left[A \mid I_{\ell+1}\right]}=Z\left(\sum_{i=0}^{\ell} \sum_{j=0}^{n} \lambda_{i} z_{j}^{\prime} x_{i j}\right)$ and $L:=\rho\left(\left[A \mid I_{\ell+1}\right]\right) \subset Z\left(\sum_{j=0}^{n} z_{i}^{\prime} x_{j}\right)$. In particular, $z:=\left(z_{0}^{\prime}, \ldots, z_{n-\ell-1}^{\prime}\right)$ is not zero. By (3.8), every $M \in T_{Z, A}$ satisfies $\sum_{i=0}^{\ell} \sum_{j=0}^{n-\ell-1} \lambda_{i} z_{j} M_{i j}=0$. Since $Z$ is a hypersurface, we have shown (3.9).

Secondly, for $A \in Z$ general, we assume that $(3.9)$ holds for some $\lambda \in \mathbb{A}^{\ell+1}$ and $z \in \mathbb{A}^{n-\ell}$. We set $p:=\left[A \mid I_{\ell+1}\right]^{T} \lambda, z^{\prime}:=\left[I_{n-\ell} \mid-A^{T}\right]^{T} z \in \mathbb{A}^{n+1} \backslash\{0\}$ and $H:=Z\left(\sum_{j=0}^{n} z_{j}^{\prime} x_{j}\right) \subset \mathbb{A}^{n+1}$. Note that $L:=\rho\left(\left[A \mid I_{\ell+1}\right]\right) \subset H$. The differential of $\rho \circ \iota$ at $A$ is an isomorphism of $T_{Z, A}$ with $T_{\Sigma, L}$. By Corollary 2.10, every $\varphi \in T_{\Sigma, L}$ satisfies $\varphi(p) \in H / L$. Since $\Sigma$ is a hypersurface, we have shown that $T_{\Sigma, L}=$ $\left\{\varphi \in \operatorname{Hom}\left(L, \mathbb{A}^{n+1} / L\right) \mid \varphi(p) \in H / L\right\}$. This shows that $\Sigma$ is coisotropic.

Finally, we turn to characterizations of coisotropy in Plücker coordinates. Cayley [18] had already realized that an irreducible hypersurface $\Sigma \subset \operatorname{Gr}\left(1, \mathbb{P}^{3}\right)$ with defining polynomial $Q$ in Plücker coordinates is coisotropic if and only if the following polynomial vanishes everywhere on $\Sigma$ :

$$
\forall L \in \Sigma:\left(\frac{\partial Q}{\partial q_{01}} \cdot \frac{\partial Q}{\partial q_{23}}-\frac{\partial Q}{\partial q_{02}} \cdot \frac{\partial Q}{\partial q_{13}}+\frac{\partial Q}{\partial q_{03}} \cdot \frac{\partial Q}{\partial q_{12}}\right)(\operatorname{pl}(L))=0 .
$$

This follows directly from the affine or Stiefel characterization of coisotropy given in Propositions 3.25 and 3.24. Now we provide a generalization of Cayley's result to $\operatorname{Gr}\left(1, \mathbb{P}^{n}\right)$ for $n \geq 3$.

For a homogeneous irreducible polynomial $Q$ in dual Plücker coordinates of 
$\operatorname{Gr}\left(1, \mathbb{P}^{n}\right)$ and for $0 \leq j<k<i<m \leq n$, define

$$
R_{j k i m}^{Q}:=\frac{\partial Q}{\partial q_{j k}} \cdot \frac{\partial Q}{\partial q_{i m}}-\frac{\partial Q}{\partial q_{j i}} \cdot \frac{\partial Q}{\partial q_{k m}}+\frac{\partial Q}{\partial q_{j m}} \cdot \frac{\partial Q}{\partial q_{k i}} .
$$

Note that this polynomial of degree $2(\operatorname{deg} Q-1)$ is a differential version of the usual Plücker relations. To allow permutations of the indices, define $R_{\pi(j k i m)}^{Q}:=$ $\operatorname{sgn}(\pi) R_{j k i m}^{Q}$ for $\pi \in S_{4}$.

Theorem 3.26. Let $n \geq 3$, and let $\Sigma \subset \operatorname{Gr}\left(1, \mathbb{P}^{n}\right)$ be an irreducible hypersurface, given by a homogeneous polynomial $Q$ in dual Plücker coordinates. Then $\Sigma$ is coisotropic if and only if for all $0 \leq i<m \leq n$, the following polynomial in dual Plücker coordinates vanishes everywhere on $\Sigma$ :

$$
\forall L \in \Sigma:\left(\sum_{0 \leq j<k \leq n, j, k \notin\{i, m\}} q_{j k} R_{j k i m}^{Q}\right)(\operatorname{pl}(L))=0 .
$$

Proof. This proof relies on Proposition 3.24. Let $\mu$ be the map that sends a $2 \times(n+1)$-matrix to its maximal minors, such that $\mu(A)_{i j}=a_{0 i} a_{1 j}-a_{0 j} a_{1 i}$ denotes the minor given by the $i$-th and $j$-th column of a matrix $A$. For shorter notation, write $\beta_{i j}:=\frac{\partial Q}{\partial q_{i j}} \circ \mu$, as well as $q_{i j}=\mu(\cdot)_{i j}$. This will be used to compute the $2 \times 2$-minors of $J_{Q \circ \mu}$. For this, pick two columns with indices $0 \leq i<m \leq n$. The set of remaining column indices is denoted by $S:=\{0, \ldots, n\} \backslash\{i, m\}$. The chain rule for partial derivatives gives

$$
\frac{\partial(Q \circ \mu)}{\partial a_{0 i}}=-\sum_{j \in S} \beta_{j i} a_{1 j}-\beta_{m i} a_{1 m}, \quad \frac{\partial(Q \circ \mu)}{\partial a_{1 m}}=\sum_{j \in S} \beta_{j m} a_{0 j}+\beta_{i m} a_{0 i} .
$$

Hence, the $(2 \times 2)$-minor of $J_{Q \circ \mu}$ given by the columns $i$ and $m$ equals

$$
\begin{aligned}
& \frac{\partial(Q \circ \mu)}{\partial a_{0 i}} \cdot \frac{\partial(Q \circ \mu)}{\partial a_{1 m}}-\frac{\partial(Q \circ \mu)}{\partial a_{1 i}} \cdot \frac{\partial(Q \circ \mu)}{\partial a_{0 m}} \\
= & a_{0 i} a_{1 m} \beta_{i m}^{2}+\sum_{j \in S} a_{0 j} a_{1 m} \beta_{i m} \beta_{j m}-\sum_{j \in S} a_{0 i} a_{1 j} \beta_{i m} \beta_{j i}-\sum_{j, k \in S} a_{0 k} a_{1 j} \beta_{j i} \beta_{k m} \\
& -a_{0 m} a_{1 i} \beta_{i m}^{2}+\sum_{j \in S} a_{0 j} a_{1 i} \beta_{i m} \beta_{j i}-\sum_{j \in S} a_{0 m} a_{1 j} \beta_{i m} \beta_{j m}+\sum_{j, k \in S} a_{0 j} a_{1 k} \beta_{j i} \beta_{k m} \\
= & q_{i m} \beta_{i m}^{2}+\sum_{j \in S} q_{j m} \beta_{i m} \beta_{j m}+\sum_{j \in S} q_{j i} \beta_{i m} \beta_{j i}+\sum_{j, k \in S, j \neq k} q_{j k} \beta_{j i} \beta_{k m} \\
= & \beta_{i m}\left(\sum_{0 \leq j<k \leq n} q_{j k} \beta_{j k}-\sum_{j, k \in S, j<k} q_{j k} \beta_{j k}\right)+\sum_{j, k \in S, j<k} q_{j k} \beta_{j i} \beta_{k m}-\sum_{j, k \in S, j<k} q_{j k} \beta_{k i} \beta_{j m} \\
= & \beta_{i m} \sum_{0 \leq j<k \leq n} q_{j k} \beta_{j k}-\sum_{j, k \in S, j<k} q_{j k}\left(\beta_{j k} \beta_{i m}-\beta_{j i} \beta_{k m}+\beta_{j m} \beta_{k i}\right)
\end{aligned}
$$




$$
=\beta_{i m} \sum_{0 \leq j<k \leq n} q_{j k} \beta_{j k}-\sum_{j, k \in S, j<k} q_{j k} R_{j k i m}^{Q}
$$

Since $Q$ is homogeneous, we have $\sum_{0 \leq j<k \leq n}\left(q_{j k} \beta_{j k}\right)(M)=0$ for all $M \in Z(Q \circ \mu)$. Now the theorem follows from Proposition 3.24 .

Example 3.27. For $n=3$, the above theorem yields exactly Cayley's differential characterization in (3.10): $R_{0123}^{Q}(\operatorname{pl}(L))=0$ for all $L \in \Sigma$. For $n=4$, one gets the following ten polynomials in dual Plücker coordinates:

$$
\begin{array}{cc}
q_{01} R_{0134}^{Q}+q_{02} R_{0234}^{Q}+q_{12} R_{1234^{\prime}}^{Q} & q_{01} R_{0124}^{Q}-q_{03} R_{0234}^{Q}-q_{13} R_{1234^{\prime}}^{Q} \\
q_{01} R_{0123}^{Q}+q_{04} R_{0234}^{Q}+q_{14} R_{1234^{\prime}}^{Q} & -q_{02} R_{0124}^{Q}-q_{03} R_{0134}^{Q}+q_{23} R_{1234^{\prime}}^{Q} \\
-q_{02} R_{0123}^{Q}+q_{04} R_{0134}^{Q}-q_{24} R_{1234^{\prime}}^{Q} & q_{03} R_{0123}^{Q}+q_{04} R_{0124}^{Q}+q_{34} R_{1234^{\prime}}^{Q} \\
q_{12} R_{0124}^{Q}+q_{13} R_{0134}^{Q}+q_{23} R_{0234^{\prime}}^{Q} & q_{12} R_{0123}^{Q}-q_{14} R_{0134}^{Q}-q_{24} R_{0234^{\prime}}^{Q} \\
-q_{13} R_{0123}^{Q}-q_{14} R_{0124}^{Q}+q_{34} R_{0234^{\prime}}^{Q} & q_{23} R_{0123}^{Q}+q_{24} R_{0124}^{Q}+q_{34} R_{0134}^{Q}
\end{array}
$$

\subsection{Cayley Variety}

Chow forms of space curves and Hurwitz forms of surfaces in $\mathbb{P}^{3}-$ which are all cases of coisotropic hypersurfaces in $\operatorname{Gr}\left(1, \mathbb{P}^{3}\right)$ - were already studied by Cayley [18]. Therefore the variety $\mathcal{C}\left(\ell, d, \mathbb{P}^{n}\right)$ of all coisotropic forms of degree $d$ in the coordinate ring of $\operatorname{Gr}\left(\ell, \mathbb{P}^{n}\right)$ is called Cayley variety in the following. Its subvariety $\mathrm{Ch}\left(\ell, d, \mathbb{P}^{n}\right)$ of all Chow forms was introduced by Chow and van der Waerden [23] and is called Chow variety. The problem of recognizing the Chow forms among all coisotropic forms is addressed in [43, Ch. 4, Sec. 3]. This goes already back to Green and Morrison [46], who gave explicit equations for the Chow variety. We present a definitive computational solution for the smallest non-trivial case, namely for cycles of dimension one and degree two in $\mathbb{P}^{3}$.

The Chow form of a cycle of degree two is a quadratic form in the Plücker coordinates of $\operatorname{Gr}\left(1, \mathbb{P}^{3}\right)$. Quadratic forms in Plücker coordinates form a projective space $\mathbb{P}^{19}$. The Chow variety we are interested in, denoted $\mathrm{Ch}\left(1,2, \mathbb{P}^{3}\right)$, is the set of all Chow forms in that $\mathbb{P}^{19}$. The aim of this section is to make the concepts in [18, 23, 46] and [43, Ch. 4.3] completely explicit. All ideals and Maple computations discussed in this section are contained in the folder section3-7 at

https://github.com/kathlenkohn/thesis-material

The Cayley variety $\mathcal{C}\left(1,2, \mathbb{P}^{3}\right)$. We start with the nine-dimensional subvariety $\mathcal{C}\left(1,2, \mathbb{P}^{3}\right)$ of $\mathbb{P}^{19}$ whose points are the coisotropic quadrics in $\operatorname{Gr}\left(1, \mathbb{P}^{3}\right)$. By Theorem 3.11, this decomposes as the Chow variety and the variety of Hurwitz forms, representing lines that are tangent to a quadric surface in $\mathbb{P}^{3}$. The generic 
quadric in $\operatorname{Gr}\left(1, \mathbb{P}^{3}\right)$ is written as a generic quadratic form

$$
Q(q)=q \cdot\left[\begin{array}{llllll}
c_{0} & c_{1} & c_{2} & c_{3} & c_{4} & c_{5} \\
c_{1} & c_{6} & c_{7} & c_{8} & c_{9} & c_{10} \\
c_{2} & c_{7} & c_{11} & c_{12} & c_{13} & c_{14} \\
c_{3} & c_{8} & c_{12} & c_{15} & c_{16} & c_{17} \\
c_{4} & c_{9} & c_{13} & c_{16} & c_{18} & c_{19} \\
c_{5} & c_{10} & c_{14} & c_{17} & c_{19} & c_{20}
\end{array}\right] \cdot q^{T} .
$$

The quadric $Q(q)$ is an element in $V:=\mathbb{C}[q]_{2} / \mathbb{C}\left\{q_{01} q_{23}-q_{02} q_{13}+q_{03} q_{12}\right\} \simeq$ $\mathbb{C}^{21} / \mathbb{C}$. Hence, $c=\left(c_{0}, c_{1}, \ldots, c_{20}\right)$ serves as homogeneous coordinates on $\mathbb{P}^{19}=$ $\mathbb{P}(V)$, which - due to the Plücker relation - need to be understood modulo

$$
c_{5} \mapsto c_{5}+\lambda, c_{9} \mapsto c_{9}-\lambda, c_{12} \mapsto c_{12}+\lambda .
$$

The coordinate ring $\mathbb{Q}[V]$ is a subring of $\mathbb{Q}\left[c_{0}, c_{1}, \ldots, c_{20}\right]$, namely it is the invariant ring of the additive group action (3.13). Hence $\mathbb{Q}[V]$ is the polynomial ring in 20 variables $c_{0}, c_{1}, c_{2}, c_{3}, c_{4}, c_{5}-c_{12}, c_{6}, c_{7}, c_{8}, c_{9}+c_{12}, c_{10}, c_{11}, c_{13}, \ldots, c_{20}$.

We are interested in the $c^{\prime}$ s that lead to coisotropic hypersurfaces of $\operatorname{Gr}\left(1, \mathbb{P}^{3}\right)$. By (3.10), the quadric hypersurface $\{Q(q)=0\}$ in $\operatorname{Gr}\left(1, \mathbb{P}^{3}\right)$ is coisotropic if and only if there exist $s, t \in \mathbb{C}$ such that

$$
\frac{\partial Q}{\partial q_{01}} \cdot \frac{\partial Q}{\partial q_{23}}-\frac{\partial Q}{\partial q_{02}} \cdot \frac{\partial Q}{\partial q_{13}}+\frac{\partial Q}{\partial q_{03}} \cdot \frac{\partial Q}{\partial q_{12}}=s \cdot Q+t \cdot\left(q_{01} q_{23}-q_{02} q_{13}+q_{03} q_{12}\right)
$$

Equivalently, the vector $(t, s,-1)^{T}$ is in the kernel of the $21 \times 3$-matrix in Figure 3.2. The $3 \times 3$-minors of this matrix are all in the subring $\mathbb{Q}[V]$. The coisotropic ideal $I$ is the ideal of $\mathbb{Q}[V]$ generated by these minors. The subscheme $Z(I)$ of $\mathbb{P}^{19}=\mathbb{P}(V)$ represents all coisotropic hypersurfaces $\{Q=0\}$ of degree two in $\operatorname{Gr}\left(1, \mathbb{P}^{3}\right)$. Using computations with Maple and Macaulay2, we found that $I$ has codimension 10, degree 92 and is minimally generated by 175 cubics. Besides, $Z(I)$ is the reduced union of three components, of dimensions nine, eight and five.

Proposition 3.28. The coisotropic ideal is the intersection of three prime ideals:

$$
I=P_{\text {Hurwitz }} \cap P_{\text {Chowlines }} \cap P_{\text {Squares. }}
$$

So, $I$ is radical. The prime $P_{\text {Hurwitz }}$ has codimension 10 and degree 92 , it is minimally generated by 20 quadrics, and its variety $Z\left(P_{\text {Hurwitz }}\right)$ consists of Hurwitz forms of quadric surfaces in $\mathbb{P}^{3}$. The prime $P_{\text {ChowLines }}$ has codimension 11 and degree 140 , it is minimally generated by 265 cubics, and $Z\left(P_{\text {ChowLines }}\right)$ consists of Chow forms of pairs of lines in $\mathbb{P}^{3}$. The prime $P_{\text {Squares }}$ has codimension 14 and degree 32 , it is minimally generated by 84 quadrics, and $Z\left(P_{\text {Squares }}\right)$ consists of all quadrics $Q(q)$ that are squares modulo the Plücker relation.

From our geometric perspective, the third prime $P_{\text {Squares }}$ is extraneous. Theorem 3.11 concerns irreducible hypersurfaces, and the identification of Chow 


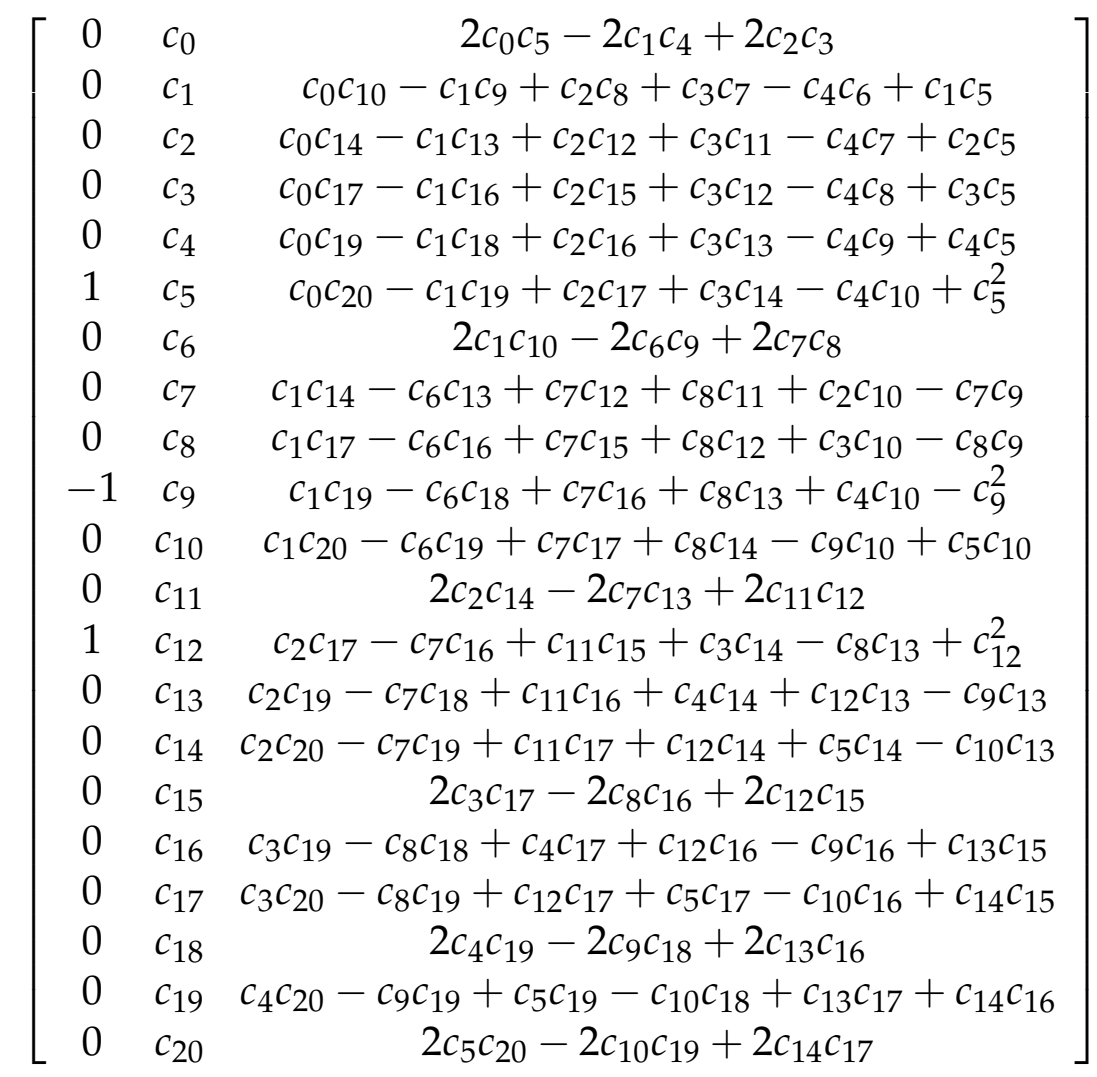

Figure 3.2: This matrix has rank $\leq 2$ if and only if the quadric given by $c$ is coisotropic.

forms within the coisotropic hypersurfaces in [43, Ch. 4, Thm. 3.22] assumes the corresponding polynomial to be squarefree. With this, the following would be the correct ideal for the Cayley variety in $\mathbb{P}^{19}$ :

$$
P_{\text {Hurwitz }} \cap P_{\text {Chowlines }}=\left(I: P_{\text {Squares }}\right) \text {. }
$$

This means that the reduced coisotropic quadrics in $\operatorname{Gr}\left(1, \mathbb{P}^{3}\right)$ are either Chow forms of curves or Hurwitz forms of surfaces. The ideal in (3.15) has codimension 10 , degree 92 , and is minimally generated by 175 cubics and 20 quartics in $\mathbb{Q}[V]$.

Remark 3.29. According to [16, Thm. 4.2], the variety $Z(I)$ is isomorphic to the variety of all quadratic polynomials $Q$ in the dual Plücker coordinates such that

$$
\left(q_{01} q_{23}-q_{02} q_{13}+q_{03} q_{12}\right) \mid\left(\frac{\partial Q}{\partial q_{01}} \cdot \frac{\partial Q}{\partial q_{23}}-\frac{\partial Q}{\partial q_{02}} \cdot \frac{\partial Q}{\partial q_{13}}+\frac{\partial Q}{\partial q_{03}} \cdot \frac{\partial Q}{\partial q_{12}}\right) .
$$

Moreover, this variety is defined by quadratic equations. In fact, analogously to our computations above, we see that it is the zero locus of the ideal $I^{\prime}$ generated by the $2 \times 2$-minors of the $21 \times 2$-matrix that is obtained by deleting the middle column of the matrix in Figure 3.2. Besides, $Z(I)$ is the image of $Z\left(I^{\prime}\right)$ under 
the projection whose center is the Plücker relation. The ideal $I^{\prime}$ has codimension 11 in the 20-dimensional space $\mathbb{P}\left(\mathbb{C}[q]_{2}\right)$. Furthermore, it has degree 92 and is minimally generated by 20 quadrics.

The Chow variety $\operatorname{Ch}\left(1,2, \mathbb{P}^{3}\right)$. Now we study the Chow variety $\operatorname{Ch}\left(1,2, \mathbb{P}^{3}\right)$ of one-dimensional algebraic cycles of degree two in $\mathbb{P}^{3}$. By [43, Ch. 4, Ex. 1.3], the Chow variety $\mathrm{Ch}\left(1,2, \mathbb{P}^{3}\right)$ is the union of two irreducible components of dimension eight in $\mathbb{P}^{19}$, one corresponding to planar quadrics and the other to pairs of lines. Formally, this means that

$$
\operatorname{Ch}\left(1,2, \mathbb{P}^{3}\right)=Z\left(P_{\text {ChowConic }}\right) \cup Z\left(P_{\text {ChowLines }}\right),
$$

where $P_{\text {ChowConic }}$ is the homogeneous prime ideal in $\mathbb{Q}[V]$ whose variety comprises the Chow forms of irreducible curves of degree two in $\mathbb{P}^{3}$. The ideal $P_{\text {ChowConic }}$ has codimension 11 and degree 92, and it is minimally generated by 21 quadrics and 35 cubics. The radical ideal $P_{\text {ChowConic }} \cap P_{\text {ChowLines }}$ has codimension 11, degree $232=92+140$, and it is minimally generated by 230 cubics.

Since $\mathrm{Ch}\left(1,2, \mathbb{P}^{3}\right)$ should be contained in the Cayley variety, it seems that $P_{\text {ChowConic }}$ is missing from the decomposition (3.14). Here is the explanation:

Proposition 3.30. Every Chow form of a plane conic in $\mathbb{P}^{3}$ is contained in the Zariski closure of the set of all quadratic Hurwitz forms. In symbols, $P_{\text {Hurwitz }} \subset P_{\text {ChowConic }}$ and thus $Z\left(P_{\text {ChowConic }}\right) \subset Z\left(P_{\text {Hurwitz }}\right)$.

Proof. Our first proof is by computer: just check the inclusion of ideals in Macaulay2. For a conceptual proof, we consider a $4 \times 4$-symmetric matrix $M=M_{0}+\varepsilon M_{1}$, where $\operatorname{rank}\left(M_{0}\right)=1$. By [101, eqn. (1)], the Hurwitz form of the corresponding quadric surface in $\mathbb{P}^{3}$ is $Q(q)=q\left(\wedge_{2} M\right) q^{T}$. Divide by $\varepsilon$ and let $\varepsilon \rightarrow 0$. The limit is the Chow form of the plane conic defined by restricting $M_{1}$ to $\operatorname{ker}\left(M_{0}\right) \simeq \mathbb{P}^{2}$. This type of degeneration is familiar from the study of complete quadrics [30]. Proposition 3.30 explains why the locus of irreducible curves is not visible in (3.14).

Gel'fand, Kapranov and Zelevinsky [43, Ch. 4.3] introduce a class of differential forms in order to discriminate Chow forms among all coisotropic hypersurfaces. In their setup, these forms represent the integrability of the $\alpha$ distribution $\mathcal{E}_{\alpha, Z}$. We shall apply the tools of computational commutative algebra to shed some light on the characterization of Chow forms via integrability of $\alpha$-distributions.

For this, we use dual affine coordinates instead of Plücker coordinates. We express the quadrics $Q$ in (3.12) in terms of the local coordinates $a_{2}, a_{3}, b_{2}, b_{3}$, by substituting the Plücker coordinates with the minors of $\left[\begin{array}{llll}1 & 0 & a_{2} & a_{3} \\ 0 & 1 & b_{2} & b_{3}\end{array}\right]$ as in (2.7). We 
consider the following differential 1-forms on $\mathbb{A}^{4}$ :

$$
\begin{array}{ll}
\alpha_{1}^{1}:=\frac{\partial Q}{\partial a_{2}} d a_{2}+\frac{\partial Q}{\partial a_{3}} d a_{3}, \quad \alpha_{2}^{1}:=\frac{\partial Q}{\partial a_{2}} d b_{2}+\frac{\partial Q}{\partial a_{3}} d b_{3}, \\
\alpha_{1}^{2}:=\frac{\partial Q}{\partial b_{2}} d a_{2}+\frac{\partial Q}{\partial b_{3}} d a_{3}, \quad \alpha_{2}^{2}:=\frac{\partial Q}{\partial b_{2}} d b_{2}+\frac{\partial Q}{\partial b_{3}} d b_{3} .
\end{array}
$$

By taking wedge products, we derive the 16 differential 4-forms

$$
d Q \wedge d \alpha_{j}^{i} \wedge \alpha_{l}^{k}=q_{i j k l} \cdot d a_{2} \wedge d a_{3} \wedge d b_{2} \wedge d b_{3} \quad \text { for } i, j, k, l \in\{1,2\} .
$$

Here the expressions $q_{i j k l}$ are certain polynomials in $\mathbb{Q}[V]\left[a_{2}, a_{3}, b_{2}, b_{3}\right]$.

Theorems 3.19 and 3.22 in [43, Ch. 4.3] state that a squarefree coisotropic quadric $Q$ is a Chow form if and only if all 16 coefficients $q_{i j k l}$ are multiples of $Q$. By taking normal forms of the polynomials $q_{i j k l}$ modulo the principal ideal $\langle Q\rangle$, we obtain a collection of 720 homogeneous polynomials in c. Among these, 58 have degree three, 340 have degree four, and 322 have degree five. The aforementioned result implies that these 720 polynomials cut out $\mathrm{Ch}\left(1,2, \mathbb{P}^{3}\right)$ as a subset of $\mathbb{P}^{19}$.

The integrability ideal $J \subset \mathbb{Q}[V]$ is generated by these 720 polynomials and their analogues from other affine charts of the Grassmannian, obtained by permuting columns in $\left[\begin{array}{llll}1 & 0 & a_{2} & a_{3} \\ 0 & 1 & b_{2} & b_{3}\end{array}\right]$. We know that $Z(J)=\mathrm{Ch}\left(1,2, \mathbb{P}^{3}\right)$ holds settheoretically. Maple, Macaulay2 and Magma verified for us that it holds schemetheoretically:

Proposition 3.31. The integrability ideal $J$ is minimally generated by 210 cubics. Writing $\mathfrak{m}$ for the irrelevant ideal $\left\langle c_{0}, c_{1}, \ldots, c_{20}\right\rangle$ of $\mathbb{Q}[V]$, we have

$$
\sqrt{J}=(J: \mathfrak{m})=P_{\text {ChowConic }} \cap P_{\text {ChowLines }} \cap P_{\text {Squares }} .
$$

Generalizations. In principal, Theorem 3.26 gives a method to compute the vanishing ideal of the Cayley variety $\mathcal{C}\left(1, d, \mathbb{P}^{n}\right)$ for $n \geq 3$. For positive integers $N$ and $D$, denote by $\left(\left(\begin{array}{l}N \\ D\end{array}\right)\right):=\left({ }_{D}^{N+D-1}\right)$ the multiset coefficient, i.e., the number of monomials of degree $D$ in $N$ variables.

Corollary 3.32. Consider the Cayley variety $\mathcal{C}\left(1, d, \mathbb{P}^{n}\right) \subset \mathbb{P}\left(\mathbb{C}\left[\operatorname{Gr}\left(1, \mathbb{P}^{n}\right)\right]_{d}\right)$, and let $c$ be a vector of homogeneous coordinates on $\mathbb{P}\left(\mathbb{C}\left[\operatorname{Gr}\left(1, \mathbb{P}^{n}\right)\right]_{d}\right)$. There are $\left(\begin{array}{c}n+1 \\ 2\end{array}\right)$ matrices of size

$$
\left(\left(\begin{array}{c}
n+1 \\
2 \\
2 d-1
\end{array}\right)\right) \times\left[1+\left(\left(\left(\begin{array}{c}
n+1 \\
2 \\
d-1
\end{array}\right)\right)\right)+\left(\begin{array}{c}
n+1 \\
4
\end{array}\right) \cdot\left(\left(\begin{array}{c}
n+1 \\
2 \\
2 d-3
\end{array}\right)\right)\right]
$$


whose entries are polynomials in $c$, such that the ideal generated by the maximal minors of these matrices defines - up to saturation - the Cayley variety $\mathcal{C}\left(1, d, \mathbb{P}^{n}\right)$. Moreover, these minors have degree

$$
2+\left(\left(\begin{array}{c}
n+1 \\
2 \\
d-1
\end{array}\right)\right)
$$

in the $\operatorname{dim}\left(\mathbb{C}\left[\operatorname{Gr}\left(1, \mathbb{P}^{n}\right)\right]_{d}\right)$ many unknowns $c$.

Proof. Let $Q$ be a general homogeneous polynomial of degree $d$ in the dual Plücker coordinates $q_{i j}$ of $\operatorname{Gr}\left(1, \mathbb{P}^{n}\right)$. Denote the coefficient vector of the polynomial $Q$ by $c$. The entries of $c$ serve as homogeneous coordinates on $\mathbb{P}\left(\mathbb{C}\left[\operatorname{Gr}\left(1, \mathbb{P}^{n}\right)\right]_{d}\right)$, although - due to the Plücker relations - they are not independent unknowns. The characterization in Theorem 3.26 states that the equation

$$
C_{i, m}:=\sum_{0 \leq j<k \leq n, j, k \notin\{i, m\}} q_{j k} R_{j k i m}^{Q}
$$

vanishes everywhere on the hypersurface of $\operatorname{Gr}\left(1, \mathbb{P}^{n}\right)$ defined by $Q$, for all $0 \leq$ $i<m \leq n$. Equivalently, the polynomial $C_{i, m}$ is contained in the radical of the ideal generated by $Q$ and the Plücker relations. Under the assumption that this ideal is already radical, we get the condition

$$
C_{i, m}-F^{(d-1)} \cdot Q-\sum_{0 \leq \alpha<\beta<\gamma<\delta \leq n} G_{\alpha \beta \gamma \delta}^{(2 d-3)} \cdot \mathcal{R}_{\alpha \beta \gamma \delta}=0,
$$

where $\mathcal{R}_{\alpha \beta \gamma \delta}$ denotes the quadratic Plücker relation $q_{\alpha \beta} q_{\gamma \delta}-q_{\alpha \gamma} q_{\beta \delta}+q_{\alpha \delta} q_{\beta \gamma}$, and $G_{\alpha \beta \gamma \delta}^{(2 d-3)}$ and $F^{(d-1)}$ are homogeneous polynomials of degree $2 d-3$ and $d-1$, respectively. In our above computation of $\mathcal{C}\left(1,2, \mathbb{P}^{3}\right)$, condition (3.21) reduces to $R_{0123}^{Q}-s \cdot Q-t \cdot \mathcal{R}_{0123}$ for constants $s$ and $t$.

Let $a$ denote the coefficient vector of $F^{(d-1)}$, and let $b$ denote the vector of all coefficients of all $G_{\alpha \beta \gamma \delta}^{(2 d-3)}$. The coefficient of each monomial of (3.21), where the variables are the Plücker coordinates $q_{i j}$, has quadratic terms in $c$ (coming from $\left.C_{i, m}\right)$, multilinear terms in $a$ and $c$ (coming from $F^{(d-1)} \cdot Q$ ), and linear terms in $b$ (coming from $\sum G_{\alpha \beta \gamma \delta}^{(2 d-3)} \cdot \mathcal{R}_{\alpha \beta \gamma \delta}$ ). Hence, we can represent such a coefficient as a vector: the quadratic terms in $c$ are the first entry. For each coefficient in $a$ we add an entry, namely the corresponding linear form in $c$. Finally, we add the constant factor of each coefficient in $b$.

In this way, we get a vector for each monomial in (3.21). Let $M_{i, m}$ be the matrix whose rows are given by these vectors. To sum up, the rows of $M_{i, m}$ are indexed by the monomials of (3.21), its columns are indexed by the entries in the vector $(1, a, b)$, and the entries of $M_{i, m}$ are (at most quadratic) polynomials in $c$ : the first column contains quadrics, the columns corresponding to $a$ consist of linear forms, and the remaining columns (corresponding to $b$ ) contain con- 
stants. In particular, the matrix $M_{i, m}$ has size (3.19), and its maximal minors have degree (3.20) in $c$. In our above computation of $\mathcal{C}\left(1,2, \mathbb{P}^{3}\right)$, we get only one matrix $M$ with 3 columns and 21 rows. This matrix is given explicitly in Figure 3.2 (but with columns in reversed order as described here).

The condition (3.21) is equivalent to that the vector $(1, a, b)$ is contained in the kernel of the matrix $M_{i, m}$. Hence, for all $0 \leq i<m \leq n$, the maximal minors of $M_{i, m}$ give basic equations for the vanishing ideal of the Cayley variety $\mathcal{C}\left(1, d, \mathbb{P}^{n}\right)$, but one still has to do some careful computational work to compute the actual vanishing ideal. There are three reasons for this. First, the maximal minors of $M_{i, m}$ also capture vectors in the kernel of $M_{i, m}$ that are of the form $(0, a, b)$. Thus one still has to saturate by the minors of the matrix that is obtained by deleting the first column from $M_{i, m}$. Note that this saturation was not needed in our above computation of $\mathcal{C}\left(1,2, \mathbb{P}^{3}\right)$, since it cannot happen that the kernel of $M$ contains vectors of the form $(0, a, b)$. Secondly, we assumed the ideal generated by $Q$ and the Plücker relations to be radical. Therefore, this method might not characterize all coisotropic forms. Finally, the maximal minors of the matrices $M_{i, m}$ already lead to extraneous factors that arise since Theorem 3.26 requires $Q$ to be irreducible. In particular, all squares trivially satisfy the condition (3.21).

Example 3.33. Consider the Cayley variety $\mathcal{C}\left(1,3, \mathbb{P}^{4}\right)$ of cubic coisotropic forms in $\operatorname{Gr}\left(1, \mathbb{P}^{4}\right)$. We have the ten equations in (3.11) of degree 5 in the 10 variables $q_{i j}$. For each such equation, condition (3.21) contains 2002 monomials. The quadric $F^{(2)}$ has 55 monomials and the cubics $G_{\alpha \beta \gamma \delta}^{(3)}$ have 220 monomials each. This leads to ten matrices with 2002 rows and $1156=1+55+5 \cdot 220$ columns. The first column of each matrix consists of quadratic forms in $c$, the next 55 columns contain linear forms in $c$, and the remaining columns have only constants. The maximal minors of these matrices are thus $10 \cdot\left(\begin{array}{l}2002 \\ 1156\end{array}\right)$ equations of degree 57 in the 220 unknowns $c$, which are in fact just $175=\operatorname{dim} \mathbb{C}\left[\operatorname{Gr}\left(1, \mathbb{P}^{4}\right)\right]_{3}$ independent unknowns due to the Plücker relations. Hence, the computation of the vanishing ideal of the Cayley variety $\mathcal{C}\left(1,3, \mathbb{P}^{4}\right)$ is a hard computational task.

\subsection{Transitive Action on Tangent Spaces}

In this section, we work over the real numbers $\mathbb{R}$ and study the concept of transitive action on tangent spaces introduced by Bürgisser and Lerario in [14].

The orthogonal group $O(n+1)$ acts transitively on $\operatorname{Gr}\left(\ell, \mathbb{P}_{\mathbb{R}}^{n}\right)$. For an element $g \in O(n+1)$, let $g_{*}: \operatorname{Gr}\left(\ell, \mathbb{P}_{\mathbb{R}}^{n}\right) \rightarrow \operatorname{Gr}\left(\ell, \mathbb{P}_{\mathbb{R}}^{n}\right)$ denote the corresponding action and $D_{L g_{*}}: T_{\operatorname{Gr}\left(\ell, \mathbb{P}_{\mathbb{R}}^{n}\right), L} \rightarrow T_{\operatorname{Gr}\left(\ell, \mathbb{P}_{\mathbb{R}}^{n}\right), g_{*}(L)}$ its derivative at $L \in \operatorname{Gr}\left(\ell, \mathbb{P}_{\mathbb{R}}^{n}\right)$.

Definition 3.34. Let $\Sigma \subset \operatorname{Gr}\left(\ell, \mathbb{P}_{\mathbb{R}}^{n}\right)$ be a subvariety. We say that $\Sigma$ has transitive action on its tangent spaces if, for general points $L_{1}, L_{2} \in \Sigma$, there is $g \in O(n+1)$ such that $g_{*}\left(L_{1}\right)=L_{2}$ and $D_{L_{1}} g_{*}\left(T_{\Sigma, L_{1}}\right)=T_{\Sigma, L_{2}}$. 
Given subvarieties of $\operatorname{Gr}\left(\ell, \mathbb{P}_{\mathbb{R}}^{n}\right)$ with transitive action on their tangent spaces, one can compute the expected volume of the intersection of $O(n+1)$-translates of the given subvarieties [14, Thm. 3.15]. This is one of the key ingredients of the propobabilistic Schubert calculus presented in [14]. In particular, the authors of [14] show that coisotropic hypersurfaces have transitive action on their tangent spaces. For completeness, we repeat their proof here.

Lemma 3.35 ([14, Lem. 3.7]). A coisotropic hypersurface $\Sigma \subset \operatorname{Gr}\left(\ell, \mathbb{P}_{\mathbb{R}}^{n}\right)$ has transitive action on its tangent spaces.

Proof. Let $L_{1}, L_{2} \in \operatorname{Reg}(\Sigma)$. For $i \in\{1,2\}$, we denote by $L_{i}{ }^{\perp} \subset \mathbb{R}^{n+1}$ the orthogonal complement of $\boldsymbol{L}_{\boldsymbol{i}}$. We use the canonical identifications

$$
N_{\Sigma, L_{i}}^{*} \subset \operatorname{Hom}\left(\mathbb{R}^{n+1} / \boldsymbol{L}_{\boldsymbol{i}}, \boldsymbol{L}_{\boldsymbol{i}}\right) \cong \boldsymbol{L}_{\boldsymbol{i}} \otimes\left(\mathbb{R}^{n+1} / \boldsymbol{L}_{\boldsymbol{i}}\right)^{*} \cong \boldsymbol{L}_{\boldsymbol{i}} \otimes \boldsymbol{L}_{\boldsymbol{i}}{ }^{\perp}
$$

Since $\Sigma$ is coisotropic, there are unit length vectors $u_{i} \in L_{\boldsymbol{i}}$ and $v_{i} \in \boldsymbol{L}_{\boldsymbol{i}}{ }^{\perp}$ such that $N_{\Sigma, L_{i}}^{*}$ is spanned by $u_{i} \otimes v_{i}$. There is $g \in O(n+1)$ such that $g_{*} L_{1}=L_{2}$, $g\left(u_{1}\right)=u_{2}$, and $g\left(v_{1}\right)=v_{2}$. This shows that $D_{L_{1}} g_{*}$ maps $N_{\Sigma, L_{1}}^{*}$ to $N_{\Sigma, L_{2}}^{*}$, so it maps $T_{\Sigma, L_{1}}$ to $T_{\Sigma, L_{2}}$.

Remark 3.36. In Section 7.1, we study isotropic curves. These are curves in Grassmannians whose tangent lines at smooth points are spanned by rank one homomorphisms. Every isotropic curve has transitive action on its tangent spaces. The proof for this assertion is analogous to the proof of Lemma 3.35.

The authors of [14] asked if all hypersurfaces of $\operatorname{Gr}\left(\ell, \mathbb{P}_{\mathbb{R}}^{n}\right)$ with transitive action on their tangent spaces are coisotropic. We show now that this is not the case by providing a counterexample in Proposition 3.37. Antonio Lerario helped with the geometric interpretation of the example as we present it here. For this, we recall the notion of principal angles between subspaces of $\mathbb{R}^{n+1}$, which was first introduced by Jordan [58]. Let us consider two linear subspaces $U_{1}, U_{2} \subset \mathbb{R}^{n+1}$. We set $m:=\min \left\{\operatorname{dim} U_{1}, \operatorname{dim} U_{2}\right\}$, choose an orthonormal basis for each subspace, and denote by $A_{i} \in \mathbb{R}^{\operatorname{dim}\left(U_{i}\right) \times(n+1)}$ the matrix whose rows are the basis vectors of $U_{i}$, for $i \in\{1,2\}$. We write $0 \leq \sigma_{1}, \ldots, \sigma_{m} \leq 1$ for the singular values of $A_{1} A_{2}^{T}$. The principal angles between $U_{1}$ and $U_{2} \operatorname{are} \theta_{j}:=\arccos \sigma_{j}$, for $j \in\{1, \ldots, m\}$. Note that this definition is independent of our choice of the orthogonal bases.

We now study the special case of the Grassmannian of lines in $\mathbb{P}^{3}$. The FubiniStudy metric on $\operatorname{Gr}\left(1, \mathbb{P}_{\mathbb{R}}^{3}\right)$ is defined as follows: for $L_{1}, L_{2} \in \operatorname{Gr}\left(1, \mathbb{P}_{\mathbb{R}}^{3}\right)$, we consider the principal angles $\theta_{1}, \theta_{2}$ between $L_{1}$ and $L_{2}$, and set

$$
d_{\mathrm{FS}}\left(L_{1}, L_{2}\right):=\arccos \left(\cos \theta_{1} \cdot \cos \theta_{2}\right) .
$$

Note that $d_{\mathrm{FS}}\left(L_{1}, L_{2}\right)=\arccos \left(\operatorname{det} A_{1} A_{2}^{T}\right)$, where the rows of the $2 \times 4$-matrices $A_{1}$ and $A_{2}$ form again orthonormal bases for $L_{1}$ and $L_{2}$. We denote the set of lines with the same distance to two given distinct lines $L_{1}, L_{2} \in \operatorname{Gr}\left(1, \mathbb{P}_{\mathbb{R}}^{3}\right)$ by

$$
\Xi_{L_{1}, L_{2}}:=\left\{L \in \operatorname{Gr}\left(1, \mathbb{P}_{\mathbb{R}}^{3}\right) \mid d_{\mathrm{FS}}\left(L, L_{1}\right)=d_{\mathrm{FS}}\left(L, L_{2}\right)\right\}
$$


Proposition 3.37. For $L_{1}, L_{2} \in \operatorname{Gr}\left(1, \mathbb{P}_{\mathbb{R}}^{3}\right)$ such that $L_{2}$ is the orthogonal complement of $\boldsymbol{L}_{\mathbf{1}}$, we have that $\Xi_{L_{1}, L_{2}}$ is a non-coisotropic hyperplane of $\operatorname{Gr}\left(1, \mathbb{P}_{\mathbb{R}}^{3}\right)$ which has transitive action on its tangent spaces.

Proof. Let $M \in O(4)$ be such that its first two rows are an orthonormal basis for $L_{1}$ and its last two rows are an orthonormal basis for $L_{2}$. Moreover, we consider $L \in \operatorname{Gr}\left(1, \mathbb{P}_{\mathbb{R}}^{3}\right)$ and a $4 \times 2$-matrix $A$ whose columns are an orthonormal basis for $L$. Then $L \in \Xi_{L_{1}, L_{2}}$ if and only if the first and the last $2 \times 2$-minor of $M A$ are equal. Hence, the linear change of coordinates given by $M$ maps $\Xi_{L_{1}, L_{2}}$ to

$$
\left\{L \in \operatorname{Gr}\left(1, \mathbb{P}_{\mathbb{R}}^{3}\right) \mid q_{01}(L)=q_{23}(L)\right\},
$$

where $q_{i j}(L)$ is the $(i, j)$-th dual Plücker coordinate of $L$. Thus, we may assume

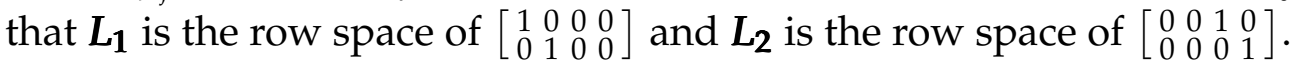

Now we describe the tangent space of $\Xi_{L_{1}, L_{2}}$ at a point $L$. Note that the image of the Grassmannian $\operatorname{Gr}\left(1, \mathbb{P}_{\mathbb{R}}^{3}\right)$ under its Plücker embedding and the hyperplane $\mathcal{H}:=Z\left(q_{01}-q_{23}\right) \subset \mathbb{P}_{\mathbb{R}}^{5}$ intersect transversely. Hence, the tangent space $T_{\Xi_{L_{1}, L}, L}$ is the preimage of $T_{\mathcal{H}, q(L)}$ under the differential of the Plücker embedding at $L$. We pick an orthonormal basis $(a, b)$ for $L$ and use Corollary 2.11 to derive that $T_{\Xi_{L_{1}, L_{2}}, L}$ is the image of

$$
\left\{\varphi \in \operatorname{Hom}\left(\boldsymbol{L}, \mathbb{R}^{4}\right) \mid=\begin{array}{r}
\left(\varphi(a)_{0} b_{1}-\varphi(a)_{1} b_{0}\right)+\left(a_{0} \varphi(b)_{1}-a_{1} \varphi(b)_{0}\right) \\
\left(\varphi(a)_{2} b_{3}-\varphi(a)_{3} b_{2}\right)+\left(a_{2} \varphi(b)_{3}-a_{3} \varphi(b)_{2}\right)
\end{array}\right\}
$$

under the map $\operatorname{Hom}\left(\boldsymbol{L}, \mathbb{R}^{4}\right) \rightarrow \operatorname{Hom}\left(\boldsymbol{L}, \mathbb{R}^{4} / \boldsymbol{L}\right)$ which is the composition with the canonical projection $\pi: \mathbb{R}^{4} \rightarrow \mathbb{R}^{4} / L$. Thus, the one-dimensional conormal space $N_{\Xi_{L_{1}, L_{2}}, L}^{*}$ is spanned by a map $\psi \in \operatorname{Hom}\left(\mathbb{R}^{4} / L, L\right)$ such that $\psi \circ \pi$ is given by the matrix

$$
\left[\begin{array}{cccc}
b_{1} & -b_{0} & -b_{3} & b_{2} \\
-a_{1} & a_{0} & a_{3} & -a_{2}
\end{array}\right]
$$

with respect to the standard basis of $\mathbb{R}^{4}$ and the basis $(a, b)$ of $L$. Note that (3.22) has rank two, which shows that $\Xi_{L_{1}, L_{2}}$ is not coisotropic. Moreover, the rows of (3.22) form an orthonormal basis of the orthogonal complement $\boldsymbol{L}^{\perp}$ of $\boldsymbol{L}$.

If we now consider a second point $L^{\prime} \in \Xi_{L_{1}, L_{2}}$ with an orthonormal basis $\left(a^{\prime}, b^{\prime}\right)$ of $L^{\prime}$, there is $g \in O(4)$ with $g(a)=a^{\prime}, g(b)=b^{\prime}, g\left(b_{1},-b_{0},-b_{3}, b_{2}\right)^{T}=$ $\left(b_{1}^{\prime},-b_{0}^{\prime},-b_{3}^{\prime}, b_{2}^{\prime}\right)^{T}$, and $g\left(-a_{1}, a_{0}, a_{3},-a_{2}\right)^{T}=\left(-a_{1}^{\prime}, a_{0}^{\prime}, a_{3}^{\prime},-a_{2}^{\prime}\right)^{T}$. In particular, $D_{L} g_{*}$ maps $N_{\Xi_{L_{1}, L_{2}}, L}^{*}$ to $N_{\Xi_{L_{1}, L_{2}}, L^{\prime}}^{*}$ so it maps $T_{\Xi_{L_{1}, L_{2}}, L}$ to $T_{\Xi_{L_{1}, L_{2}}, L^{\prime}}$.

\subsection{Computations}

A Macaulay2 package for calculating coisotropic hypersurfaces and recovering their underlying projective varieties can be obtained at

https://github.com/kathlenkohn/thesis-material 
with the file name Coisotropy.m2. To use the package, the user can simply start Macaulay2 from the same directory where the package file was saved and then use the command loadPackage "Coisotropy". After that, the following commands are available:

dualVariety I: Computes the ideal of the projectively dual variety of the projective variety given by the ideal $\mathrm{I}$.

polarDegrees I: Computes a list whose $i$-th entry is the degree of the $i$-th coisotropic hypersurface of the projective variety given by the ideal I. This is done by computing the multidegree of the conormal variety, as described in Section 3.2 .

coisotropicForm $(I, i)$ : Returns the i-th coisotropic form in primal Plücker coordinates of the projective variety given by the ideal $\mathrm{I}$. The computation of this form follows essentially Definition 3.2 .

isCoisotropic $(\mathrm{Q}, \mathrm{k}, \mathrm{n})$ : Checks if a hypersurface in $\operatorname{Gr}\left(\mathrm{k}, \mathbb{P}^{\mathrm{n}}\right)$ is coisotropic. The hypersurface is given by a polynomial $Q$ in primal Plücker coordinates. This is implemented by using the characterization of coisotropy in Proposition 3.25 . recoverVar $(\mathrm{Q}, \mathrm{k}, \mathrm{n})$ : Computes the ideal of the underlying projective variety of a given coisotropic hypersurface in $\operatorname{Gr}\left(\mathrm{k}, \mathbb{P}^{\mathrm{n}}\right)$, which is defined by a polynomial Q in primal Plücker coordinates. This computation uses the Cayley trick in Proposition 3.4 .

dualToPrimal $(\mathrm{Q}, \mathrm{k}, \mathrm{n})$ : Transforms a polynomial $\mathrm{Q}$ in dual Plücker coordinates of the Grassmannian $\mathrm{Gr}\left(\mathrm{k}, \mathbb{P}^{\mathrm{n}}\right)$ to a polynomial in primal Plücker coordinates. This can be used to perform the change of coordinates (2.4) before calling one of the above commands that require primal Plücker coordinates.

primalToDual $(\mathrm{Q}, \mathrm{k}, \mathrm{n})$ : Reverse transformation to dualToPrimal.

After having studied coisotropic hypersurfaces in detail, we will not restrict ourselves to varieties of codimension one in the following chapters. 


\section{Coisotropic Varieties}

In this chapter, we aim to generalize the notion of coisotropy to subvarieties of Grassmannians with codimension larger than one. There are two natural candidates for such a notion: either we require every homomorphism in the conormal spaces of the given variety to have rank at most one, or we require each conormal space to be spanned by rank one homomorphisms. If we want our condition for coisotropy to be Zariski closed, we need to adjust the latter alternative slightly: thus, we require each conormal space to be in the Zariski closure of the set of all linear spaces that are spanned by homomorphisms of rank one. To give a more precise definition, we introduce Grassmann secant varieties.

\subsection{Grassmann secant varieties}

We define the following analogue of secant varieties in Grassmannians. For an irreducible variety $X \subset \mathbb{P}^{n}$ and $k \in \mathbb{Z}_{\geq 0}$, we set

$$
\operatorname{Sec}_{k}^{0}(X):=\left\{L \in \operatorname{Gr}\left(k, \mathbb{P}^{n}\right) \mid L=\operatorname{span}(L \cap X)\right\}
$$

and let $\operatorname{Sec}_{k}(X) \subset \operatorname{Gr}\left(k, \mathbb{P}^{n}\right)$ denote its Zariski closure, called the $k$-th Grassmann secant variety of $X$. Grassmann secant varieties have recently played a role in the study of tensor rank and Waring's problem [11, 15, 22, 21], but they have also been studied on their own right [20, 24, 27]. In [24], Ciliberto and Cools show, for every irreducible and non-degenerate variety $X \subset \mathbb{P}^{n}$, that

$$
\operatorname{dim} \operatorname{Sec}_{k}(X) \geq(k+1) \operatorname{dim} X, \quad \text { if } \operatorname{Sec}_{k}(X) \neq \operatorname{Gr}\left(k, \mathbb{P}^{n}\right) .
$$

This implies immediately the following.

Lemma 4.1. Let $X \subset \mathbb{P}^{n}$ be an irreducible nondegenerate variety. If $k \geq \operatorname{codim} X$, then $\operatorname{Sec}_{k}(X)=\operatorname{Gr}\left(k, \mathbb{P}^{n}\right)$.

Proof. If $k \geq \operatorname{codim} X$, then $(k+1) \operatorname{dim} X \geq(k+1)(n-k)=\operatorname{dim} \operatorname{Gr}\left(k, \mathbb{P}^{n}\right)$ and the assertion follows from (4.1).

We also present an alternative proof: we show by induction on $k$ that a general $L \in \operatorname{Gr}\left(k, \mathbb{P}^{n}\right)$ is spanned by $L \cap X$. For the induction beginning, we consider $k=\operatorname{codim} X$ : a general $L \in \operatorname{Gr}\left(k, \mathbb{P}^{n}\right)$ intersects $X$ at $\operatorname{deg}(X)$ points and is spanned by those points [19, Thm. 59]. For the induction step, we assume $k>\operatorname{codim} X$. We consider a general $L \in \operatorname{Gr}\left(k, \mathbb{P}^{n}\right)$ and two general hyperplanes $H_{1}, H_{2} \subset L$. By induction hypothesis, both $H_{1}$ and $H_{2}$ are spanned by 
their respective intersection with $X$. Since $L$ is spanned by $H_{1}$ and $H_{2}$, it is also spanned by $L \cap X$.

Before defining coisotropic varieties formally, we state another key property of Grassmann secant varieties for our purposes.

Lemma 4.2. Consider an irreducible variety $X \subset \mathbb{P}^{n}$. For $L_{1} \in \operatorname{Sec}_{k_{1}}(X)$ and $L_{2} \in \operatorname{Sec}_{k_{2}}(X)$, we have that $L_{1}+L_{2} \in \operatorname{Sec}_{\operatorname{dim}\left(L_{1}+L_{2}\right)}(X)$.

Proof. Let $k:=\operatorname{dim}\left(L_{1}+L_{2}\right)$. For $i \in\{1,2\}$, we write $S_{i}:=\operatorname{Sec}_{k_{i}}(X)$ and $S_{i}^{0}:=\operatorname{Sec}_{k_{i}}^{0}(X)$. In addition, we set

$$
U:=\left\{\left(L_{1}^{\prime}, L_{2}^{\prime}\right) \in S_{1}^{0} \times S_{2}^{0} \mid \operatorname{dim}\left(L_{1}^{\prime}+L_{2}^{\prime}\right)=k\right\} .
$$

For every $\left(L_{1}^{\prime}, L_{2}^{\prime}\right) \in U$, we have $L_{1}^{\prime}+L_{2}^{\prime} \in \operatorname{Sec}_{k}(X)$. Since $S_{i}^{0}$ is dense in $S_{i}$ and $O:=\left\{\left(L_{1}^{\prime}, L_{2}^{\prime}\right) \in S_{1} \times S_{2} \mid \operatorname{dim}\left(L_{1}^{\prime}+L_{2}^{\prime}\right)=k\right\}$ is a dense and open subset of $Z:=\left\{\left(L_{1}^{\prime}, L_{2}^{\prime}\right) \in S_{1} \times S_{2} \mid \operatorname{dim}\left(L_{1}^{\prime}+L_{2}^{\prime}\right) \leq k\right\}$, it follows that $U=\left(S_{1}^{0} \times S_{2}^{0}\right) \cap O$ is dense in $Z$. Thus, the Zariski closure of the image of the rational map

$$
\begin{aligned}
& Z \rightarrow \operatorname{Gr}\left(k, \mathbb{P}^{n}\right), \\
&\left(L_{1}^{\prime}, L_{2}^{\prime}\right) \longmapsto L_{1}^{\prime}+L_{2}^{\prime},
\end{aligned}
$$

which is defined on all of $O$, is contained in $\operatorname{Sec}_{k}(X)$.

\subsection{Two Notions of Coisotropy}

In this thesis, we are mainly interested in Grassmann secant varieties of Segre varieties. For two vector spaces $U$ and $W$, a key player for our definition of coisotropic varieties is

$$
\operatorname{Sec}_{k}(\operatorname{Seg}(U, W))=\overline{\{L \mid L=\operatorname{span}(L \cap \operatorname{Seg}(U, W))\}} \subset \operatorname{Gr}(k, \mathbb{P}(\operatorname{Hom}(U, W))) .
$$

Definition 4.3. An irreducible subvariety $\Sigma \subset \operatorname{Gr}\left(\ell, \mathbb{P}^{n}\right)$ of codimension $c \geq 1$ is coisotropic if, for every $L \in \operatorname{Reg}(\Sigma)$, the conormal space of $\Sigma$ at $L$ is spanned by rank one homomorphisms or is in the Zariski closure of the set of such spaces, i.e.,

$$
\mathbb{P}\left(N_{\Sigma, L}^{*}\right) \in \operatorname{Sec}_{c-1}\left(\operatorname{Seg}\left(\mathbb{A}^{n+1} / \boldsymbol{L}, \boldsymbol{L}\right)\right) .
$$

Moreover, $\Sigma$ is strongly coisotropic if, for every $L \in \operatorname{Reg}(\Sigma)$, the rank of every homomorphism in the conormal space of $\Sigma$ at $L$ is at most one, i.e.,

$$
\mathbb{P}\left(N_{\Sigma, L}^{*}\right) \subset \operatorname{Seg}\left(\mathbb{A}^{n+1} / \boldsymbol{L}, \boldsymbol{L}\right) .
$$

Example 4.4. Let $C \subset \mathbb{P}^{3}$ be a nondegenerate irreducible curve. The Zariski closure $\operatorname{Sec}(C):=\operatorname{Sec}_{1}(C) \subset \operatorname{Gr}\left(1, \mathbb{P}^{3}\right)$ of the set of all secant lines of $C$ (i.e., lines which intersect $C$ twice) is a surface. By Proposition 3.10 and (3.3), we can 
compute the tangent and conormal space of $\operatorname{Sec}(C)$ at a general secant line $L$ :

$$
T_{\operatorname{Sec}(C), L}=\left\{\varphi \in \operatorname{Hom}\left(\boldsymbol{L}, \mathbb{A}^{4} / \boldsymbol{L}\right) \mid \forall i \in\{1,2\}: \varphi\left(\boldsymbol{x}_{\boldsymbol{i}}\right) \subset \boldsymbol{H}_{\boldsymbol{i}} / \boldsymbol{L}\right\},
$$

where $C \cap L=\left\{x_{1}, x_{2}\right\}$ and $H_{i}:=\mathbb{T}_{C, x_{i}}+L$ is a tangent plane to $C$ for $i \in\{1,2\}$. Thus, $T_{\operatorname{Sec}(C), L}$ is spanned by two homomorphisms $\varphi_{1}, \varphi_{2}: L \rightarrow \mathbb{A}^{4} / L$ with $\operatorname{ker} \varphi_{1}=\boldsymbol{x}_{\mathbf{1}}, \operatorname{im} \varphi_{1}=\boldsymbol{H}_{\mathbf{2}} / \boldsymbol{L}, \operatorname{ker} \varphi_{2}=\boldsymbol{x}_{\mathbf{2}}$, and $\operatorname{im} \varphi_{2}=\boldsymbol{H}_{\mathbf{1}} / \boldsymbol{L}$. So the conormal space $N_{\operatorname{Sec}(C), L}^{*}$ is spanned by two rank one homomorphisms $\psi_{1}, \psi_{2}: \mathbb{A}^{4} / \boldsymbol{L} \rightarrow \boldsymbol{L}$ with $\operatorname{ker} \psi_{i}=\boldsymbol{H}_{\boldsymbol{i}} / \boldsymbol{L}$ and $\operatorname{im} \psi_{i}=\boldsymbol{x}_{\boldsymbol{i}}$ for $i \in\{1,2\}$. This shows that $\operatorname{Sec}(C)$ is coisotropic. But since $L$ was chosen generally, we have $\operatorname{rank}\left(\psi_{1}+\psi_{2}\right)=2$ and $\operatorname{Sec}(C)$ is not strongly coisotropic.

Our leading questions for the next chapters are the following:

1. Can we characterize (strongly) coisotropic varieties by underlying projective varieties analogously to Theorem 3.11 ?

2. Are singular loci of coisotropic varieties again coisotropic?

3. Do we need the Zariski closure in the definition of coisotropy, or could we define a variety to be coisotropic if its conormal space at a general point is spanned by rank one homomorphisms?

We give a full classification of all strongly coisotropic varieties in Section 4.3 . Our result is a direct generalization of Theorem 3.11. Moreover, we answer the third question in Section 4.4 we show that we need the Zariski closure in the definition of coisotropic varieties, by presenting geometrically meaningful examples of coisotropic varieties, whose conormal spaces are not spanned by rank one homomorphisms. Since the second question seems to be harder to answer, we study iterated singular loci of the coisotropic hypersurfaces in $\operatorname{Gr}\left(1, \mathbb{P}^{3}\right)$ explicitly in Chapter 5. We present an application of those iterated singular loci to algebraic vision in Chapter 6 .

In this thesis, we will not give a full classification of all coisotropic varieties. Instead, we state two simple observations towards such a classification in the remainder of this section. First, all varieties of low enough dimension are coisotropic, which follows immediately from Lemma 4.1 .

Corollary 4.5. Every subvariety of $\operatorname{Gr}\left(\ell, \mathbb{P}^{n}\right)$ of dimension at most $n-1$ is coisotropic.

Proof. Let $\Sigma \subset \operatorname{Gr}\left(\ell, \mathbb{P}^{n}\right)$ be a subvariety with $\operatorname{dim} \Sigma \leq n-1$. The codimension $c$ of $\Sigma$ in $\operatorname{Gr}\left(\ell, \mathbb{P}^{n}\right)$ is at least $(\ell+1)(n-\ell)-(n-1)=\ell(n-\ell-1)+1$. Moreover, the codimension of $\operatorname{Seg}\left(\mathbb{A}^{n+1} / L, L\right)$ in $\mathbb{P}\left(\operatorname{Hom}\left(\mathbb{A}^{n+1} / L, L\right)\right)$ is $\ell(n-\ell-1)$ for every $L \in \operatorname{Gr}\left(\ell, \mathbb{P}^{n}\right)$. By Lemma 4.1, we have

$$
\operatorname{Sec}_{c-1}\left(\operatorname{Seg}\left(\mathbb{A}^{n+1} / L, L\right)\right)=\operatorname{Gr}\left(c-1, \mathbb{P}\left(\operatorname{Hom}\left(\mathbb{A}^{n+1} / L, L\right)\right)\right) .
$$

In particular, $\Sigma$ is coisotropic. 
Example 4.6. An irreducible subvariety of $\operatorname{Gr}\left(1, \mathbb{P}^{3}\right)$ is coisotropic if and only if it is a point, a curve, a surface, the Chow hypersurface of a curve in $\mathbb{P}^{3}$, or the Hurwitz hypersurface of a surface in $\mathbb{P}^{3}$ (see Theorem 3.11/ Example 3.12.

Secondly, we will see that generically transverse intersections of coisotropic varieties are coisotropic again, which follows from Lemma 4.2. We say that two subvarieties $X_{1}, X_{2} \subset Y$ intersect transversely at a point $x \in X_{1} \cap X_{2}$ if $X_{1}, X_{2}$ and $Y$ are all smooth at $x$ and $T_{X_{1}, x}+T_{X_{2}, x}=T_{Y, x}$. The subvarieties $X_{1}, X_{2} \subset Y$ are said to intersect generically transversely if every irreducible component of $X_{1} \cap X_{2}$ contains a point where $X_{1}$ and $X_{2}$ are transverse.

Corollary 4.7. If two coisotropic subvarieties $\Sigma_{1}, \Sigma_{2} \subset \operatorname{Gr}\left(\ell, \mathbb{P}^{n}\right)$ intersect generically transversely, then every irreducible component of $\Sigma_{1} \cap \Sigma_{2}$ is coisotropic.

Proof. Let $\Sigma$ be a non-empty irreducible component of $\Sigma_{1} \cap \Sigma_{2}$, and let $L \in \Sigma$ be a general point. We have $\operatorname{dim} T_{\Sigma, L}=\operatorname{dim} \Sigma \geq \operatorname{dim} \Sigma_{1}+\operatorname{dim} \Sigma_{2}-\operatorname{dim} \operatorname{Gr}\left(\ell, \mathbb{P}^{n}\right)$. Since $\Sigma_{1}$ and $\Sigma_{2}$ intersect transversely at $L$ and $T_{\Sigma, L} \subset T_{\Sigma_{1}, L} \cap T_{\Sigma_{2}, L}$, we also have

$$
\operatorname{dim} T_{\Sigma, L} \leq \operatorname{dim}\left(T_{\Sigma_{1}, L} \cap T_{\Sigma_{2}, L}\right)=\operatorname{dim} \Sigma_{1}+\operatorname{dim} \Sigma_{2}-\operatorname{dim} \operatorname{Gr}\left(\ell, \mathbb{P}^{n}\right) .
$$

Thus, $T_{\Sigma, L}=T_{\Sigma_{1}, L} \cap T_{\Sigma_{2}, L}$. Besides, the transverse intersection at $L$ implies $N_{\Sigma_{1}, L}^{*} \cap N_{\Sigma_{2}, L}^{*}=\{0\}$, which shows that both $N_{\Sigma_{1}, L}^{*}+N_{\Sigma_{2}, L}^{*}$ and $N_{\Sigma, L}^{*}$ have the same dimension (namely $\left.2 \operatorname{dim} \operatorname{Gr}\left(\ell, \mathbb{P}^{n}\right)-\operatorname{dim} \Sigma_{1}-\operatorname{dim} \Sigma_{2}\right)$. As $N_{\Sigma_{1}, L}^{*}+N_{\Sigma_{2}, L}^{*}$ is contained in $N_{\Sigma, L}^{*}$, we can apply Lemma 4.2 to the equality

$$
\mathbb{P}\left(N_{\Sigma, L}^{*}\right)=\mathbb{P}\left(N_{\Sigma_{1}, L}^{*}\right)+\mathbb{P}\left(N_{\Sigma_{2}, L}^{*}\right) \in \operatorname{Sec}_{c-1}\left(\operatorname{Seg}\left(\mathbb{A}^{n+1} / \boldsymbol{L}, \boldsymbol{L}\right)\right),
$$

where $c$ is the codimension of $\Sigma$ in $\operatorname{Gr}\left(\ell, \mathbb{P}^{n}\right)$. Since $L \in \Sigma$ was chosen generally, we have shown $\mathbb{P}\left(N_{\Sigma, L}^{*}\right) \in \operatorname{Sec}_{c-1}\left(\operatorname{Seg}\left(\mathbb{A}^{n+1} / L, L\right)\right)$ for every $L \in \operatorname{Reg}(\Sigma)$.

Example 4.8. Similarly, self-intersections of coisotropic hypersurfaces are coisotropic as well. For instance, consider a nondegenerate surface $S$ in $\mathbb{P}^{4}$. The self-intersection $\operatorname{Sec}(S):=\operatorname{Sec}_{1}(S)$ of its Chow hypersurface is a four-dimensional subvariety of $\operatorname{Gr}\left(1, \mathbb{P}^{4}\right)$. This is the Zariski closure of the set of its secant lines. As in Example 4.4, we see that the conormal space of $\operatorname{Sec}(S)$ at a general secant line $L$ is spanned by two rank one homomorphisms $\psi_{1}, \psi_{2}: \mathbb{A}^{5} / \boldsymbol{L} \rightarrow \boldsymbol{L}$ with ker $\psi_{i}=\left(\mathbb{T}_{S, x_{i}}+\boldsymbol{L}\right) / \boldsymbol{L}$ and im $\psi_{i}=\boldsymbol{x}_{\boldsymbol{i}}$, where $S \cap L=\left\{x_{1}, x_{2}\right\}$. Hence, $\operatorname{Sec}(S)$ is coisotropic, although it is not trivially coisotropic by Corollary 4.5 .

Analogously, for a general hypersurface $X \subset \mathbb{P}^{n}$ of degree at least four, the self-intersection $\operatorname{Bit}(X)$ of its Hurwitz hypersurface is coisotropic. More specifically, $\operatorname{Bit}(X)$ is the Zariski closure of the set of all bitangent lines of $X$. As above, the conormal space of this locus at a general bitangent line $L$ is spanned by two rank one homomorphisms $\psi_{1}, \psi_{2}: \mathbb{A}^{n+1} / \boldsymbol{L} \rightarrow \boldsymbol{L}$ with $\operatorname{ker} \psi_{i}=\mathbb{T}_{\boldsymbol{X}, \boldsymbol{x}_{\boldsymbol{i}}} / \boldsymbol{L}$ and $\operatorname{im} \psi_{i}=\boldsymbol{x}_{\boldsymbol{i}}$, where the line $L$ is bitangent to $X$ at the points $x_{1}$ and $x_{2}$.

Conjecture 4.9. We conjecture that the irreducible components of the singular locus of a coisotropic variety are again coisotropic. This is supported by the following class of 
examples. We will see in Theorem 5.13 that the singular locus of the Hurwitz hypersurface of a general hypersurface $X \subset \mathbb{P}^{n}$ of degree at least $2(n-1)$ has two components: the locus of all bitangent lines and the locus of all lines, which intersect $X$ at some point with multiplicity at least three. By Example 4.8 and Theorem 4.26, both components are coisotropic.

\subsection{Strongly Coisotropic Varieties}

First examples of strongly coisotropic subvarieties of $\operatorname{Gr}\left(\ell, \mathbb{P}^{n}\right)$ are sets of linear spaces that intersect a fixed variety in $\mathbb{P}^{n}$ of dimension at most $n-\ell-1$ (see Proposition 4.14).

Definition 4.10. For an irreducible variety $X \subset \mathbb{P}^{n}$ and $0 \leq \ell \leq n-\operatorname{dim} X-1$, we define the $\ell$-th associated variety of $X$ as follows:

$$
\mathcal{G}_{\ell}(X):=\left\{L \in \operatorname{Gr}\left(\ell, \mathbb{P}^{n}\right) \mid L \cap X \neq \varnothing\right\}
$$

Example 4.11. The $(n-\operatorname{dim} X-1)$-th associated variety of $X$ is the Chow hypersurface of $X$.

Example 4.12. We consider the Segre variety $X:=\operatorname{Seg}\left(\mathbb{A}^{2}, \mathbb{A}^{4}\right) \subset \mathbb{P}(V) \cong \mathbb{P}^{7}$, where $V:=\operatorname{Hom}\left(\mathbb{A}^{2}, \mathbb{A}^{4}\right)$. It has dimension four, so $\ell$ ranges between zero and two in Definition 4.10. Clearly, $\mathcal{G}_{0}(X)=X$ and $\mathcal{G}_{1}(X)$ is the set of lines intersecting $X$. Moreover, $\mathcal{G}_{2}(X)=\mathrm{CH}_{0}(X)$.

Remark 4.13. Note that $\mathcal{G}_{\ell}(X)=\mathrm{CH}_{\ell-(n-1-\operatorname{dim} X)}(X)$ when we extend Definition 3.2 of $\mathrm{CH}_{i}(X)$ to negative $i \in\{-(n-1-\operatorname{dim} X), \ldots, 0\}$. In that way, we associate a coisotropic variety in each Grassmannian $\operatorname{Gr}\left(0, \mathbb{P}^{n}\right), \ldots, \operatorname{Gr}\left(n-1, \mathbb{P}^{n}\right)$ to $X \subset \mathbb{P}^{n}$, as we will see in Corollary 4.16

Proposition 4.14. For every irreducible variety $X \subset \mathbb{P}^{n}$ and $\ell \leq n-\operatorname{dim} X-1$, the $\ell$-th associated variety $\mathcal{G}_{\ell}(X) \subset \operatorname{Gr}\left(\ell, \mathbb{P}^{n}\right)$ of $X$ is strongly coisotropic.

Proof. We proceed as in the proof of Proposition 3.10. A general $L \in \mathcal{G}_{\ell}(X)$ intersects $X$ at exactly one point $x_{L}$, which gives us a rational map $\omega: \mathcal{G}_{\ell}(X) \rightarrow X$, $L \mapsto x_{L}$. By Corollary 2.9, we have $\left.\varphi\right|_{x_{L}}=\left(D_{L} \mathcal{\omega}(\varphi) \bmod L\right)$ for a general $L \in \mathcal{G}_{\ell}(X)$ and every $\varphi \in T_{\mathcal{G}_{\ell}(X), L}$. Since the image of $D_{L} \mathcal{W}(\varphi)$ is contained in $\mathbb{T}_{\boldsymbol{X}, \boldsymbol{x}_{\mathbf{L}}} / \boldsymbol{x}_{\mathbf{L}}$, the tangent space $T_{\mathcal{G}_{\ell}(X), L}$ is contained in

$$
V_{L}:=\left\{\varphi \in \operatorname{Hom}\left(\boldsymbol{L}, \mathbb{A}^{n+1} / \boldsymbol{L}\right) \mid \varphi\left(\boldsymbol{x}_{\boldsymbol{L}}\right) \subset\left(\mathbb{T}_{\boldsymbol{X}, \boldsymbol{x}_{\boldsymbol{L}}}+\boldsymbol{L}\right) / \boldsymbol{L}\right\}
$$

Since $L$ was chosen generally, the dimension of $\mathbb{T}_{X, x_{L}}+L$ is $\operatorname{dim} X+\ell$, which shows that $V_{L}$ has dimension $\ell(n-\ell)+\operatorname{dim} X$. This is also the dimension of the variety $\mathcal{G}_{\ell}(X)$. Hence, we have derived

$$
\begin{aligned}
T_{\mathcal{G}_{\ell}(X), L} & =V_{L} \\
& =\left\{\varphi \in T_{\operatorname{Gr}\left(\ell, \mathbb{P}^{n}\right), L} \mid \boldsymbol{x}_{\boldsymbol{L}} \subset \operatorname{ker} \varphi\right\}+\left\{\varphi \in T_{\operatorname{Gr}\left(\ell, \mathbb{P}^{n}\right), L} \mid \operatorname{im} \varphi \subset\left(\mathbf{T}_{\mathbf{X}, \boldsymbol{x}_{\mathbf{L}}}+\boldsymbol{L}\right) / \boldsymbol{L}\right\} .
\end{aligned}
$$


This shows that the conormal space of $\mathcal{G}_{\ell}(X)$ at a general point $L \in \mathcal{G}_{\ell}(X)$ contains only homomorphisms of rank one:

$N_{\mathcal{G}_{\ell}(X), L}^{*}=\left\{\varphi \in \operatorname{Hom}\left(\mathbb{A}^{n+1} / \boldsymbol{L}, \boldsymbol{L}\right) \mid\left(\mathbb{T}_{\boldsymbol{X}_{,} \boldsymbol{x}_{\boldsymbol{L}}}+\boldsymbol{L}\right) / \boldsymbol{L} \subset \operatorname{ker} \varphi, \operatorname{im} \varphi \subset \boldsymbol{x}_{\boldsymbol{L}}\right\}$.

Since $L$ was chosen generally, we have shown $\mathbb{P}\left(N_{\mathcal{G}_{\ell}(X), L}^{*}\right) \subset \operatorname{Seg}\left(\mathbb{A}^{n+1} / \boldsymbol{L}, \boldsymbol{L}\right)$ for every $L \in \operatorname{Reg}\left(\mathcal{G}_{\ell}(X)\right)$.

Remark 4.15. The duality statement in Theorem 3.13 and its proof extend to all coisotropic varieties associated to a variety $X \subset \mathbb{P}^{n}$, i.e.,

$$
\mathrm{CH}_{i}(X)^{\perp}=\mathrm{CH}_{\operatorname{dim} X-\operatorname{codim} X^{\vee}+1-i}\left(X^{\vee}\right)
$$

holds for all $i \in\{-(n-1-\operatorname{dim} X), \ldots, \operatorname{dim} X\}$. In particular, we have shown the following.

Corollary 4.16. The $i$-th coisotropic variety $\mathrm{CH}_{i}(X)$ of an irreducible variety $X \subset \mathbb{P}^{n}$ is strongly coisotropic for each $i \in\{-(n-1-\operatorname{dim} X), \ldots, \operatorname{dim} X\}$.

Proof. If $\mathrm{CH}_{i}(X)$ is a hypersurface, we apply Theorem 3.11. If $i<0$, the assertion follows from Remark 4.13 and Proposition 4.14 Finally, if $i>0$ and $\mathrm{CH}_{i}(X)$ is not a hypersurface, Corollary 3.5 and Remark 4.15 imply $\mathrm{CH}_{i}(X)^{\perp}=$ $\mathrm{CH}_{j}\left(X^{\vee}\right)$ for some $j<0$. As above, $\mathrm{CH}_{j}\left(X^{\vee}\right)$ is strongly coisotropic, which shows that $\mathrm{CH}_{i}(X)$ is also strongly coisotropic by Remark/Definition 2.6.

Example 4.17. As in Example 4.12, we consider $V:=\operatorname{Hom}\left(\mathbb{A}^{2}, \mathbb{A}^{4}\right)$ as well as $X:=\operatorname{Seg}\left(\mathbb{A}^{2}, \mathbb{A}^{4}\right)$ in $\mathbb{P}(V)$. This Segre variety is self-dual: $X^{\vee}=\operatorname{Seg}\left(\mathbb{A}^{4}, \mathbb{A}^{2}\right)$ in $\mathbb{P}\left(V^{*}\right)$. There are seven strongly coisotropic varieties associated to $X$, among which are the three coisotropic hypersurfaces depicted in the middle:

$$
\begin{array}{lll}
\mathcal{G}_{0}(X)=\mathrm{CH}_{-2}(X)=X & \mathrm{CH}_{0}(X)=\mathcal{G}_{2}(X) & \mathrm{CH}_{3}(X) \\
\mathcal{G}_{1}(X)=\mathrm{CH}_{-1}(X) & \mathrm{CH}_{1}(X) & \mathrm{CH}_{4}(X) \\
& \mathrm{CH}_{2}(X) &
\end{array}
$$

The extended duality relations (additionally to those in Theorem 3.13) read $\mathrm{CH}_{4}(X)^{\perp}=\mathrm{CH}_{-2}\left(X^{\vee}\right)=\mathcal{G}_{0}\left(X^{\vee}\right)=X^{\vee}$ as well as $\mathrm{CH}_{3}(X)^{\perp}=\mathrm{CH}_{-1}\left(X^{\vee}\right)=$ $\mathcal{G}_{1}\left(X^{\vee}\right)$.

In fact, every strongly coisotropic variety is associated to a projective variety as in Corollary 4.16. We prove this assertion in the remainder of this section.

Theorem 4.18. For an irreducible strongly coisotropic variety $\Sigma \subset \operatorname{Gr}\left(\ell, \mathbb{P}^{n}\right)$, there is an irreducible variety $X \subset \mathbb{P}^{n}$ such that $\Sigma=\mathrm{CH}_{\ell-(n-1-\operatorname{dim} X)}(X)$.

Since we have already shown this statement for coisotropic hypersurfaces in Theorem 3.11, we consider only strongly coisotropic varieties of codimension at least two in the following. For this, we use the notion of $\alpha$ - and $\beta$-spaces introduced in Definition 2.12. 
Lemma 4.19. Let $U$ and $W$ be vector spaces. Every linear space $E$ of dimension at least two which is contained in the affine cone $\{\varphi \in \operatorname{Hom}(U, W) \mid \operatorname{rank}(\varphi) \leq 1\}$ over the Segre variety $\operatorname{Seg}(U, W)$ is contained in a unique $\alpha$-or $\beta$-space.

Proof. Since the dimension of the intersection of two distinct $\alpha$-spaces is zero (similarly for $\beta$-spaces) and the intersection of an $\alpha$-with a $\beta$-space is onedimensional, it is enough to show that $E$ is contained in some $\alpha$ - or $\beta$-space.

If $E$ would neither be contained in an $\alpha$-nor in a $\beta$-space, then $E$ would con$\operatorname{tain} \varphi_{1}, \varphi_{2}, \psi_{1}, \psi_{2} \in \operatorname{Hom}(U, W) \backslash\{0\}$ with $\operatorname{ker} \varphi_{1} \neq \operatorname{ker} \varphi_{2}$ and $\operatorname{im} \psi_{1} \neq \operatorname{im} \psi_{2}$. Among these four homomorphisms are two with both, distinct kernels and distinct images, say $\chi_{1}$ and $\chi_{2}$. Let us pick $u_{1} \in \operatorname{ker} \chi_{1} \backslash \operatorname{ker} \chi_{2}$ as well as $u_{2} \in \operatorname{ker} \chi_{2} \backslash \operatorname{ker} \chi_{1}$. We see that $\left(\chi_{1}+\chi_{2}\right)\left(u_{1}\right) \in \operatorname{im} \chi_{2} \backslash\{0\}$ and analogously $\left(\chi_{1}+\chi_{2}\right)\left(u_{2}\right) \in \operatorname{im} \chi_{1} \backslash\{0\}$. Thus, the image of $\chi_{1}+\chi_{2} \in E$ must be at least two-dimensional, which contradicts that all homomorphisms in $E$ have rank at most one.

Definition/Corollary 4.20. Let $\Sigma$ be an irreducible strongly coisotropic variety of codimension at least two. Either each conormal space at a smooth point of $\Sigma$ is contained in a unique $\alpha$-space, or each conormal space at a smooth point of $\Sigma$ is contained in a unique $\beta$-space. In the first case, we call $\Sigma$ strongly coisotropic of $\alpha$-type. In the latter case, we say that $\Sigma$ is strongly coisotropic of $\beta$-type.

Corollary 4.21. Every strongly coisotropic variety in $\operatorname{Gr}\left(\ell, \mathbb{P}^{n}\right)$ of $\alpha$-type has codimension at most $\ell+1$, and every strongly coisotropic variety of $\beta$-type has codimension at most $n-\ell$.

Proof. This follows from the fact that $\alpha$-spaces in $\operatorname{Hom}\left(\mathbb{A}^{n+1} / L, L\right)$ have dimension $\ell+1$ and that the dimension of $\beta$-spaces is $n-\ell$, where $L \in \operatorname{Gr}\left(\ell, \mathbb{P}^{n}\right)$.

Lemma 4.22. A subvariety $\Sigma \subset \operatorname{Gr}\left(\ell, \mathbb{P}^{n}\right)$ is strongly coisotropic of $\beta$-type if and only if $\Sigma^{\perp} \subset \operatorname{Gr}\left(n-\ell-1,\left(\mathbb{P}^{n}\right)^{*}\right)$ is strongly coisotropic of $\alpha$-type.

Proof. First, we notice that $L$ is a smooth point of $\Sigma$ if and only if $L^{\vee}$ is a smooth point of $\Sigma^{\perp}$. Secondly, the image of $\varphi \in N_{\Sigma, L}^{*}$ is contained in a linear subspace $U \subset \boldsymbol{L}$ if and only the kernel of $\varphi^{*} \in N_{\Sigma^{\perp}, L^{\vee}}^{*}$ contains $(\boldsymbol{L} / U)^{*}$.

Corollary 4.23. Let $X \subset \mathbb{P}^{n}$ be an irreducible variety. Table 4.1 summarizes the types of the strongly coisotropic varieties associated to X (see (4.2) and Remark 4.15).

Example 4.24. For the Segre variety $X:=\operatorname{Seg}\left(\mathbb{A}^{2}, \mathbb{A}^{4}\right)$, the two associated strongly coisotropic varieties on the left of (4.3) are of $\beta$-type, the three varieties in the middle of (4.3) are its coisotropic hypersurfaces, and the two strongly coisotropic varieties on the right of (4.3) are of $\alpha$-type.

Now we will finally prove Theorem 4.18 by restricting ourselves to strongly coisotropic varieties of $\beta$-type.

Theorem 4.25. For an irreducible strongly coisotropic variety $\Sigma \subset \operatorname{Gr}\left(\ell, \mathbb{P}^{n}\right)$ of $\beta$ type, there is an irreducible variety $X \subset \mathbb{P}^{n}$ such that $\Sigma=\mathcal{G}_{\ell}(X)$. 


\begin{tabular}{ccc}
\hline$i$ & ambient $\operatorname{Grassmannian}$ of $\mathrm{CH}_{i}(X)$ & type of $\mathrm{CH}_{i}(X)$ \\
\hline$-(n-1-\operatorname{dim} X)$ & $\operatorname{Gr}\left(0, \mathbb{P}^{n}\right)$ & $\beta$-type \\
$\vdots$ & $\vdots$ & \\
-1 & $\mathrm{Gr}\left(\operatorname{codim} X-2, \mathbb{P}^{n}\right)$ & hypersurface \\
$\vdots$ & $\mathrm{Gr}\left(\operatorname{codim} X-1, \mathbb{P}^{n}\right)$ & \\
$\operatorname{dim} X-\operatorname{codim} X^{\vee}+1$ & $\operatorname{Gr}\left(\operatorname{dim} X^{\vee}, \mathbb{P}^{n}\right)$ & $\alpha$-type \\
\hline $\operatorname{dim} X-\operatorname{codim} X^{\vee}+2$ & $\operatorname{Gr}\left(\operatorname{dim} X^{\vee}+1, \mathbb{P}^{n}\right)$ & \\
$\operatorname{dim} X$ & $\operatorname{Gr}\left(n-1, \mathbb{P}^{n}\right)$ & \\
\hline
\end{tabular}

Table 4.1: Types of strongly coisotropic varieties associated to an irreducible variety $X$ in $\mathbb{P}^{n}$.

Proof. Since $\Sigma$ is strongly coisotropic of $\beta$-type, there is, for every $L \in \operatorname{Reg}(\Sigma)$, a unique $x_{L} \in L$ such that the conormal space $N_{\Sigma, L}^{*}$ is contained in the $\beta$-space $E_{\beta}\left(\boldsymbol{x}_{\boldsymbol{L}}\right)$. In other words, for every $L \in \operatorname{Reg}(\Sigma)$ and every $\varphi \in N_{\Sigma, L}^{*}$, the image of $\varphi$ is contained in the one-dimensional subspace $\boldsymbol{x}_{\boldsymbol{L}} \subset \mathbb{A}^{n+1}$ corresponding to the projective point $x_{L}$. Hence, we get a rational map $\rho: \Sigma \rightarrow \mathbb{P}^{n}$ which maps $L \in \operatorname{Reg}(\Sigma)$ to $x_{L} \in \mathbb{P}^{n}$. We denote the Zariski closure of the image of this map by $X \subset \mathbb{P}^{n}$. Note that $X$ is irreducible since $\Sigma$ is irreducible.

Moreover, for every $L \in \operatorname{Reg}(\Sigma)$, we consider the intersection of the kernels of all $\varphi \in N_{\Sigma, L}^{*}$ to find $P_{L} \in \operatorname{Gr}\left(n-\operatorname{codim} \Sigma, \mathbb{P}^{n}\right)$ with $L \subset P_{L}$ and $\boldsymbol{P}_{\boldsymbol{L}} / \boldsymbol{L} \subset \operatorname{ker} \varphi$ for every $\varphi \in N_{\Sigma, L}^{*}$. Thus, for $L \in \operatorname{Reg}(\Sigma)$, we see that

$$
N_{\Sigma, L}^{*}=\left\{\varphi \in \operatorname{Hom}\left(\mathbb{A}^{n+1} / \boldsymbol{L}, \boldsymbol{L}\right) \mid \operatorname{im} \varphi \subset \boldsymbol{x}_{\boldsymbol{L}}, \boldsymbol{P}_{\boldsymbol{L}} / \boldsymbol{L} \subset \operatorname{ker} \varphi\right\},
$$

since both spaces in (4.4) have the same dimension (namely codim $\Sigma$ ). This shows

$$
\begin{aligned}
T_{\Sigma, L} & =\left\{\varphi \in \operatorname{Hom}\left(\boldsymbol{L}, \mathbb{A}^{n+1} / \boldsymbol{L}\right) \mid \boldsymbol{x}_{\boldsymbol{L}} \subset \operatorname{ker} \varphi\right\}+\left\{\varphi \in \operatorname{Hom}\left(\boldsymbol{L}, \mathbb{A}^{n+1} / \boldsymbol{L}\right) \mid \operatorname{im} \varphi \subset \boldsymbol{P}_{\boldsymbol{L}} / \boldsymbol{L}\right\} \\
& =\left\{\varphi \in \operatorname{Hom}\left(\boldsymbol{L}, \mathbb{A}^{n+1} / \boldsymbol{L}\right) \mid \varphi\left(\boldsymbol{x}_{\mathbf{L}}\right) \subset \boldsymbol{P}_{\mathbf{L}} / \boldsymbol{L}\right\} .
\end{aligned}
$$

The differential of $\rho: \Sigma \rightarrow X$ at a general $L \in \Sigma$ is a surjection $T_{\Sigma, L} \rightarrow T_{X, x_{L}}$. By Corollary 2.9, the image of each $\psi: x_{L} \rightarrow \mathbb{A}^{n+1} / x_{L}$ in $T_{X, x_{L}}$ is contained in $\boldsymbol{P}_{\boldsymbol{L}} / \boldsymbol{x}_{\boldsymbol{L}}$. Thus, $\mathbb{T}_{X, x_{L}} \subset P_{L}$ for a general $L \in \Sigma$. In particular, the dimension of $X$ is at most $\operatorname{dim} P_{L}=n-\operatorname{codim} \Sigma$. Since we have constructed $X$ such that $\Sigma \subset \mathcal{G}_{\ell}(X)$, we derive

$$
\begin{aligned}
\operatorname{dim} \Sigma & \leq \operatorname{dim} \mathcal{G}_{\ell}(X) \leq \ell(n-\ell)+\operatorname{dim} X \\
& \leq \ell(n-\ell)+n-\operatorname{codim} \Sigma=\ell(n-\ell)+n-(\ell+1)(n-\ell)+\operatorname{dim} \Sigma \\
& =\operatorname{dim} \Sigma+\ell .
\end{aligned}
$$


This shows that $\operatorname{dim} X \geq \operatorname{dim} \Sigma-\ell(n-\ell)=n-\operatorname{codim} \Sigma-\ell$. If $\operatorname{dim} X=$ $n-\operatorname{codim} \Sigma-\ell$, then all inequalities in the first two of (4.5) are equalities and $\Sigma=\mathcal{G}_{\ell}(X)$. Hence, it is only left to show that the dimension of $X$ cannot be larger than $n-\operatorname{codim} \Sigma-\ell$.

Let us first assume that $\operatorname{dim} X=n-\operatorname{codim} \Sigma>n-\operatorname{codim} \Sigma-\ell$. In this case, we have $\ell>0, P_{L}=\mathbb{T}_{X, x_{L}}$ for a general $L \in \Sigma$, and

$$
\Sigma \subset \Sigma^{\prime}:=\overline{\left\{L \mid \exists x \in \operatorname{Reg}(X): x \in L \subset \mathbb{T}_{X, x_{L}}\right\}} \subset \operatorname{Gr}\left(\ell, \mathbb{P}^{n}\right) .
$$

This yields

$$
\begin{aligned}
\operatorname{dim} \Sigma & \leq \operatorname{dim} \Sigma^{\prime} \leq \operatorname{dim} X+\operatorname{dim} \operatorname{Gr}\left(\ell-1, \mathbb{P}^{\operatorname{dim} X-1}\right) \\
& =(n-\operatorname{codim} \Sigma)+\ell(n-\operatorname{codim} \Sigma-\ell) \\
& =\operatorname{dim} \Sigma-\ell(n-\ell-1)+\ell(\operatorname{dim} \Sigma-\ell(n-\ell)),
\end{aligned}
$$

which is, due to $\ell>0$, equivalent to $\operatorname{dim} \Sigma \geq(\ell+1)(n-\ell)-1$. The latter inequality is a contradiction to $\operatorname{codim} \Sigma \geq 2$.

Finally, we assume that $n-\operatorname{codim} \Sigma-\ell<\operatorname{dim} X<n-\operatorname{codim} \Sigma$. We define $\delta:=\operatorname{dim} X-(n-\operatorname{codim} \Sigma-\ell)=\operatorname{dim} X-\operatorname{dim} \Sigma+\ell(n-\ell)>0$. For a general $L \in \Sigma$, we have $\mathbb{T}_{X, x_{L}}+L \subset P_{L}$ and thus $\operatorname{dim}\left(\mathbb{T}_{X, x_{L}} \cap L\right) \geq \delta$. This shows that

$$
\Sigma \subset \Sigma^{\prime \prime}:=\overline{\left\{L \mid \exists x \in \operatorname{Reg}(X): x \in L, \operatorname{dim}\left(\mathbb{T}_{X, x} \cap L\right) \geq \delta\right\}} \subset \operatorname{Gr}\left(\ell, \mathbb{P}^{n}\right) .
$$

We can parametrize a general point in $\Sigma^{\prime \prime}$ by a point $x \in \operatorname{Reg}(X)$, a $\delta$-dimensional subspace $L^{\prime}$ of $\mathbb{T}_{X, x}$ passing through $x$, and an $\ell$-dimensional subspace of $\mathbb{P}^{n}$ containing $L^{\prime}$. This leads us to

$$
\begin{aligned}
\operatorname{dim} \Sigma & \leq \operatorname{dim} \Sigma^{\prime \prime} \\
& \leq \operatorname{dim} X+\operatorname{dim} \operatorname{Gr}\left(\delta-1, \mathbb{P}^{\operatorname{dim} X-1}\right)+\operatorname{dim} \operatorname{Gr}\left(\ell-\delta-1, \mathbb{P}^{n-\delta-1}\right) \\
& =\operatorname{dim} X+\delta(\operatorname{dim} X-\delta)+(\ell-\delta)(n-\ell)=: D .
\end{aligned}
$$

Note that $\ell-\delta-1 \geq 0$ due to $\operatorname{dim} X<n-\operatorname{codim} \Sigma$. By definition of $D$ and $\delta$,

$$
0 \leq D-\operatorname{dim} \Sigma=\delta(1+(\operatorname{dim} X-\delta)-(n-\ell))=-\delta(\operatorname{codim} \Sigma-1) .
$$

This is a contradiction since $\operatorname{codim} \Sigma \geq 2$ and $\delta>0$.

Proof of Theorem 4.18. If $\Sigma$ is a hypersurface, we apply Theorem 3.11. If $\Sigma$ is strongly coisotropic of $\beta$-type, the assertion follows from Theorem 4.25 and Remark 4.13. Finally, if $\Sigma$ is strongly coisotropic of $\alpha$-type, then $\Sigma^{\perp}$ is strongly coisotropic of $\beta$-type by Lemma 4.22 . Thus, $\Sigma^{\perp}=\mathrm{CH}_{\operatorname{dim} X-\ell}(X)$ for some irreducible variety $X \subset\left(\mathbb{P}^{n}\right)^{*}$. By Remark 4.15 , we get $\Sigma=\mathrm{CH}_{\ell-\left(n-1-\operatorname{dim} X^{\vee}\right)}\left(X^{\vee}\right)$. 


\subsection{Lines with Higher Contact to Hypersurfaces}

For a hypersurface $X \subset \mathbb{P}^{n}$ and $1 \leq m \leq \operatorname{deg} X$, we define

$$
\begin{aligned}
\mathcal{L}_{m}(X): & =\overline{\{L \mid \exists p \in \operatorname{Reg}(X): L \text { intersects } X \text { at } p \text { with multiplicity } m\}} \\
& \subset \operatorname{Gr}\left(1, \mathbb{P}^{n}\right) .
\end{aligned}
$$

Lines that intersect $X$ at some point with multiplicity three are known as principal tangents or as inflectional lines. Lines which intersect $X$ with multiplicity four at some point are called flecnodal. Inflectional and flecnodal lines will appear frequently in the remainder of this thesis.

Throughout this section, we consider a general hypersurface $X \subset \mathbb{P}^{n}$ of degree at least three and assume $m \leq n$. The subvariety $\mathcal{L}_{m}(X)$ of the Grassmannian of lines in $\mathbb{P}^{n}$ has codimension $m-1$ (cf. Lemma 4.28). We will show that $\mathcal{L}_{m}(X)$ is coisotropic, but that its conormal spaces are not spanned by rank one homomorphisms if $m \geq 3$ :

Theorem 4.26. If $m \geq 2$, a general $L \in \mathcal{L}_{m}(X)$ intersects $X$ at exactly one point $p_{L} \in \operatorname{Reg}(X)$ with multiplicity $m$ and

$$
\mathbb{P}\left(N_{\mathcal{L}_{m}(X), L}^{*}\right) \cap \operatorname{Seg}\left(\mathbb{A}^{n+1} / \mathbf{L}, \boldsymbol{L}\right)=\{\varphi\},
$$

where $\operatorname{im} \varphi=\boldsymbol{p}_{\boldsymbol{L}}$ and $\operatorname{ker} \varphi=\mathbf{T}_{\boldsymbol{X}, \boldsymbol{p}_{\mathbf{L}}} / \boldsymbol{L}$. Moreover, the $(m-2)$-dimensional projectivized conormal space $\mathbb{P}\left(N_{\mathcal{L}_{m}(X), L}^{*}\right)$ intersects $\operatorname{Seg}\left(\mathbb{A}^{n+1} / L, L\right)$ at $\varphi$ with multiplicity $m-1$. In particular,

$$
\mathbb{P}\left(N_{\mathcal{L}_{m}(X), L}^{*}\right) \in \operatorname{Sec}_{m-2}\left(\operatorname{Seg}\left(\mathbb{A}^{n+1} / \boldsymbol{L}, \boldsymbol{L}\right)\right)
$$

and $\mathcal{L}_{m}(X)$ is coisotropic.

Example 4.27. $\mathcal{L}_{2}(X)=\mathrm{CH}_{1}(X)$ is the Hurwitz hypersurface of $X$. For a general $L \in \mathcal{L}_{2}(X)$, we have seen in Proposition 3.10 that $\mathbb{P}\left(N_{\mathcal{L}_{2}(X), L}^{*}\right)$ consists of one projectivized homomorphism with image $\boldsymbol{p}_{L}$ and kernel $\mathbf{T}_{X, p_{L}} / \boldsymbol{L}$.

The projectivized conormal space at a general $L \in \mathcal{L}_{3}(X)$ is a tangent line to the Segre variety $\operatorname{Seg}\left(\mathbb{A}^{n+1} / L, L\right)$ at this homomorphism.

To prove Theorem 4.26, we further define

$$
\mathcal{L}_{m, p}(X):=\overline{\{L \mid L \text { intersects } X \text { at } p \text { with multiplicity } m\}} \subset \operatorname{Gr}\left(1, \mathbb{P}^{n}\right)
$$

for any $p \in \operatorname{Reg}(X)$ and denote by $\mathcal{C}_{m, p}(X) \subset \mathbb{P}^{n}$ the union of all $L \in \mathcal{L}_{m, p}(X)$.

Lemma 4.28. For a general $p \in X$, the cone $\mathcal{C}_{m, p}(X)$ has codimension $m-1$ in $\mathbb{P}^{n}$ and degree $(m-1) !$. Moreover, it is smooth everywhere, except at $p$ if $m \geq 3$.

Proof. We may assume that $p$ is the origin of an affine chart of $\mathbb{P}^{n}$. We consider the defining equation $f(x)=f_{1}(x)+f_{2}(x)+\ldots$ of $X$ in this affine chart, 
where $f_{i}$ is a homogeneous polynomial of degree $i$. In this affine chart, the cone $\mathcal{C}_{m, p}(X)$ is the zero locus of $\left\{f_{1}(x), \ldots, f_{m-1}(x)\right\}$. Since $X$ and $p$ are general, the polynomials $f_{1}, \ldots, f_{m-1}$ define smooth irreducible hypersurfaces whose projectivizations intersect transversely.

Note that $\mathcal{C}_{1, p}(X)=\mathbb{P}^{n}$ and that $\mathcal{C}_{2, p}(X)=\mathbb{T}_{X, p}$. Each cone $\mathcal{C}_{m, p}(X)$ is a hypersurface in the cone $\mathcal{C}_{m-1, p}(X)$. In particular, for a line $L \in \mathcal{L}_{m, p}(X)$, we have a flag of projective spaces

$$
p \in L \subset \mathbb{T}_{L} \mathcal{C}_{m, p}(X) \subset \mathbb{T}_{L} \mathcal{C}_{m-1, p}(X) \subset \ldots \subset \mathbb{T}_{L} \mathcal{C}_{2, p}(X)=\mathbb{T}_{X, p} \subset \mathbb{P}^{n},
$$

where $\mathbb{T}_{L} \mathcal{C}_{k, p}(X)$ is a hyperplane in $\mathbb{T}_{L} \mathcal{C}_{k-1, p}(X)$ for $2<k \leq m$. Here $\mathbb{T}_{L} \mathcal{C}_{k, p}(X)$ denotes the unique embedded tangent space of the cone $\mathcal{C}_{k, p}(X)$ along $L$. Besides, we denote by $T_{L} \mathcal{C}_{k, p}(X):=\left\{\varphi: \boldsymbol{p} \rightarrow \mathbb{A}^{n+1} / \boldsymbol{p} \mid \operatorname{im} \varphi \subset \mathbb{T}_{\boldsymbol{L}} \mathcal{C}_{\boldsymbol{k}, \boldsymbol{p}}(\boldsymbol{X}) / \boldsymbol{p}\right\}$ the corresponding subspace of $T_{\mathbb{P}^{n}, p}$.

For $m \geq 2$, we consider the rational map $\mathcal{L}_{m}(X) \rightarrow X$ which sends a general $L \in \mathcal{L}_{m}(X)$ to the unique point $p_{L} \in \operatorname{Reg}(X)$ at which $L$ intersects $X$ with multiplicity $m$. For a general $L \in \mathcal{L}_{m}(X)$, the differential of this map at $L$ is a surjection $\Phi_{L}: T_{\mathcal{L}_{m}(X), L} \rightarrow T_{X, p_{L}}$. We can prove Theorem 4.26 by computing the preimages of $\Phi_{L}$ along the flag in (4.6).

Lemma 4.29. For $m \geq 2$ and $L \in \mathcal{L}_{m}(X)$ general, we have

$$
\begin{aligned}
& \operatorname{ker} \Phi_{L}=\left\{\varphi \in \mathcal{H} \mid \boldsymbol{p}_{\boldsymbol{L}} \subset \operatorname{ker} \varphi, \operatorname{im} \varphi \subset \mathbb{T}_{\boldsymbol{L}} \mathcal{C}_{\boldsymbol{m}, \boldsymbol{p}_{\boldsymbol{L}}}(\boldsymbol{X}) / \boldsymbol{L}\right\}, \\
& \Phi_{L}^{-1}\left(T_{L, p_{L}}\right)=\left\{\varphi \in \mathcal{H} \mid \boldsymbol{p}_{\boldsymbol{L}} \subset \operatorname{ker} \varphi, \operatorname{im} \varphi \subset \mathbb{T}_{\boldsymbol{L}} \mathcal{C}_{\boldsymbol{m}-\mathbf{1}, \boldsymbol{p}_{\mathbf{L}}}(\boldsymbol{X}) / \boldsymbol{L}\right\},
\end{aligned}
$$

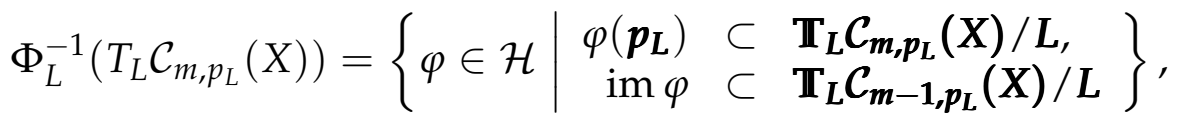

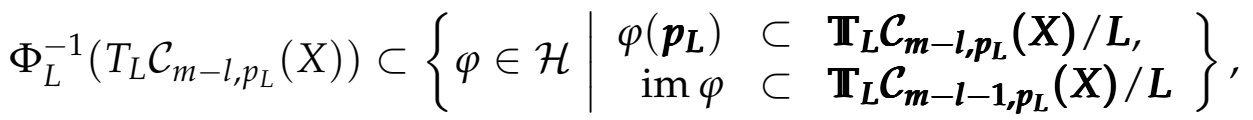

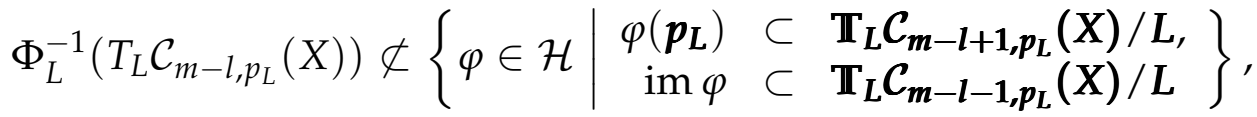

$$
\begin{aligned}
& \Phi_{L}^{-1}\left(T_{L} \mathcal{C}_{m-l, p_{L}}(X)\right) \not \subset\left\{\varphi \in \mathcal{H} \mid \begin{array}{rrr}
\varphi\left(\boldsymbol{p}_{\boldsymbol{L}}\right) & \subset & \mathbb{T}_{\boldsymbol{L}} \mathcal{C}_{\boldsymbol{m}-\boldsymbol{l}, \boldsymbol{p}_{\boldsymbol{L}}}(\boldsymbol{X}) / \boldsymbol{L}, \\
\operatorname{im} \varphi & \subset & \mathbb{T}_{\boldsymbol{L}} \mathcal{C}_{\boldsymbol{m}-\boldsymbol{l}, \boldsymbol{p}_{\boldsymbol{L}}}(\boldsymbol{X}) / \boldsymbol{L}
\end{array}\right\},
\end{aligned}
$$

where $1 \leq l \leq m-2$ and $\mathcal{H}:=\operatorname{Hom}\left(\boldsymbol{L}, \mathbb{A}^{n+1} / \boldsymbol{L}\right)$.

We prove Theorem 4.26 before we give the technical proof of Lemma 4.29 . The ideas for the computations in the next two proofs were developed together with Emre Sertöz.

Proof of Theorem 4.26 We choose coordinates for $\boldsymbol{L}$ and $\mathbb{A}^{n+1} / \boldsymbol{L}$ such that we can write every $\varphi \in \operatorname{Hom}\left(\boldsymbol{L}, \mathbb{A}^{n+1} / \boldsymbol{L}\right)$ as a matrix in $\mathbb{A}^{2 \times(n-1)}$. For $\boldsymbol{L}$, we pick a basis $\left\{e_{0}, e_{1}\right\}$ such that $e_{0} \in \boldsymbol{p}_{\boldsymbol{L}} \backslash\{0\}$ and $e_{1} \in \boldsymbol{L} \backslash \boldsymbol{p}_{\boldsymbol{L}}$. For $\mathbb{A}^{n+1} / \boldsymbol{L}$, we pick a basis $\left\{e_{2}, \ldots, e_{n}\right\}$ such that $\mathbb{T}_{\boldsymbol{L}} \mathcal{C}_{\boldsymbol{k}, p_{\mathbf{L}}}(\boldsymbol{X}) / \boldsymbol{L}$ is spanned by $e_{2}, \ldots, e_{n-k+1}$ for $2 \leq k \leq m$. The first row of a matrix in $\mathbb{A}^{2 \times(n-1)}$ encoding a map in 
$\operatorname{Hom}\left(\boldsymbol{L}, \mathbb{A}^{n+1} / \boldsymbol{L}\right)$ corresponds to $e_{0}$, the second row to $e_{1}$, and the $i$-th column corresponds to $e_{i+1}$. According to Lemma 4.29, the kernel of $\Phi_{L}$ is spanned by

$$
\left[\begin{array}{llll}
0 & 0 & \cdots & 0 \\
1 & 0 & \cdots & 0
\end{array}\right], \quad \cdots,\left[\begin{array}{lllllll}
0 & \cdots & 0 & 0 & 0 & \cdots & 0 \\
0 & \cdots & 0 & 1 & 0 & \cdots & 0
\end{array}\right]
$$

$\Phi_{L}^{-1}\left(T_{L, p_{L}}\right)$ is additionally spanned by $\quad\left[\begin{array}{lllllll}0 & \cdots & 0 & & & \\ 0 & \cdots & 0 & \cdots & 0 \\ 0 & \cdots & 0 & 1 & 0 & \cdots & 0\end{array}\right]$. $\Phi_{L}^{-1}\left(T_{L} \mathcal{C}_{m, p_{L}}(X)\right)$ is spanned by all the matrices above as well as

$$
\left[\begin{array}{llll}
1 & 0 & \cdots & 0 \\
0 & 0 & \cdots & 0
\end{array}\right], \quad \cdots,\left[\begin{array}{lllllll}
0 & \cdots & 0 & 1 & 0 & \cdots & 0 \\
0 & \cdots & 0 & 0 & 0 & \cdots & 0
\end{array}\right]
$$

$\Phi_{L}^{-1}\left(T_{L} \mathcal{C}_{m-1, p_{L}}(X)\right)$ is additionally spanned by

$$
\left[\begin{array}{cccccccc}
0 & n-m+1 \\
& \cdots & 0 & 1 & 0 & 0 & \cdots & 0 \\
0 & \cdots & 0 & 0 & C_{1} & 0 & \cdots & 0
\end{array}\right]
$$

for some non-zero constant $C_{1}$. Analogously, $\Phi_{L}^{-1}\left(T_{L} \mathcal{C}_{m-2, p_{L}}(X)\right)$ is additionally spanned by

$$
\left[\begin{array}{cccccccc}
0 & n-m+2 \\
& \cdots & 0 & 1 & 0 & 0 & \cdots & 0 \\
0 & \cdots & 0 & c_{2,1} & C_{2} & 0 & \cdots & 0
\end{array}\right]
$$

where $C_{2} \neq 0$ and $c_{2,1}$ are constants. More generally, for $1 \leq l \leq m-2$, a non-zero matrix in $\Phi_{L}^{-1}\left(T_{L} \mathcal{C}_{m-l, p_{L}}(X)\right) \backslash \Phi_{L}^{-1}\left(T_{L} \mathcal{C}_{m-l+1, p_{L}}(X)\right)$ is

$$
\left[\begin{array}{ccccccccccc}
0 & \cdots & 0 & 0 & \cdots & 0 & 1 & 0 & 0 & \cdots & 0 \\
0 & \cdots & 0 & c_{l, 1} & \cdots & c_{l, l-2} & c_{l, l-1} & C_{l} & 0 & \cdots & 0
\end{array}\right],
$$


where $C_{l} \neq 0$ and $c_{l, 1}, \ldots, c_{l, l-1}$ are some constants. In particular, for $l=m-2$, this matrix is

$$
\left[\begin{array}{cccccccc}
0 & \cdots & 0 & 0 & \cdots & 0 & 1 & 0 \\
0 & \cdots & 0 & c_{m-2,1} & \cdots & c_{m-2, m-4} & c_{m-2, m-3} & C_{m-2}
\end{array}\right] .
$$

All these matrices together span $T_{\mathcal{L}_{m}(X), L}$. Thus, the conormal space $N_{\mathcal{L}_{m}(X), L}^{*}$ is spanned by maps in $\operatorname{Hom}\left(\mathbb{A}^{n+1} / L, L\right)$ corresponding to

$$
\begin{gathered}
{\left[\begin{array}{llll}
0 & \cdots & 0 & 1 \\
0 & \cdots & 0 & 0
\end{array}\right],\left[\begin{array}{ccccccccc}
0 & \cdots & 0 & -C_{1} & -c_{2,1} & -c_{3,1} & \cdots & -c_{m-2,1} & 0 \\
0 & \cdots & 0 & 0 & 1 & 0 & \cdots & 0 & 0
\end{array}\right],} \\
\cdots,\left[\begin{array}{lllcccc}
0 & \cdots & 0 & -C_{m-3} & -c_{m-2, m-3} & 0 \\
0 & \cdots & 0 & 0 & 1 & 0
\end{array}\right],\left[\begin{array}{ccccc}
0 & \cdots & 0 & -C_{m-2} & 0 \\
0 & \cdots & 0 & 0 & 1
\end{array}\right] .
\end{gathered}
$$

Since the matrix entry in row 2 and column $n-m+1$ is zero in every matrix in $N_{\mathcal{L}_{m}(X), L}^{*}$, the only rank one matrices in $N_{\mathcal{L}_{m}(X), L}^{*}$ are scalar multiples of the first matrix in (4.13). By our choice of coordinates, the linear map $\varphi: \mathbb{A}^{n+1} / \boldsymbol{L} \rightarrow \boldsymbol{L}$ corresponding to the first matrix in 4.13 has image $p_{L}$ and kernel $\mathbb{T}_{\boldsymbol{X}, \boldsymbol{p}_{\boldsymbol{L}}} / \boldsymbol{L}$. Thus, set-theoretically the intersection of $\mathbb{P}\left(N_{\mathcal{L}_{m}(X), L}^{*}\right)$ with the Segre variety $\operatorname{Seg}\left(\mathbb{A}^{n+1} / L, L\right)$ consists just of one point as claimed in Theorem 4.26 .

We can either see from (4.13) or directly from (4.9) that

$$
N_{\mathcal{L}_{m}(X), L}^{*} \subset\left\{\psi \in \operatorname{Hom}\left(\mathbb{A}^{n+1} / \boldsymbol{L}, \boldsymbol{L}\right) \mid \mathbb{T}_{\boldsymbol{L}} \mathcal{C}_{\boldsymbol{m}, \boldsymbol{p}_{\boldsymbol{L}}}(\boldsymbol{X}) / \boldsymbol{L} \subset \operatorname{ker} \psi\right\}
$$

Hence, we can embed $N_{\mathcal{L}_{m}(X), L}^{*}$ canonically into $\operatorname{Hom}\left(\mathbb{A}^{n+1} / \mathbb{T}_{\boldsymbol{L}} \mathcal{C}_{\boldsymbol{m}, \boldsymbol{p}_{\boldsymbol{L}}}(\boldsymbol{X}), \boldsymbol{L}\right)$ and denote the image of this embedding by $N^{\prime}$. In our coordinates, this embedding simply forgets the first $n-m$ columns of the matrices in (4.13). As before, $\mathbb{P}\left(N^{\prime}\right)$ intersects the Segre variety $\operatorname{Seg}\left(\mathbb{A}^{n+1} / \mathbb{T}_{\boldsymbol{L}} \mathcal{C}_{\boldsymbol{m}, \boldsymbol{p}_{\boldsymbol{L}}}(\boldsymbol{X}), \boldsymbol{L}\right)$ set-theoretically at one point. Since the codimension of this Segre variety in its ambient space $\mathbb{P}\left(\operatorname{Hom}\left(\mathbb{A}^{n+1} / \mathbb{T}_{\boldsymbol{L}} \mathcal{C}_{\boldsymbol{m}, \boldsymbol{p}_{\boldsymbol{L}}}(\boldsymbol{X}), \boldsymbol{L}\right)\right)$ is $m-2$ and the dimension of $\mathbb{P}\left(N^{\prime}\right)$ is also $m-2$, the intersection multiplicity at the unique point of intersection is $\operatorname{deg} \operatorname{Seg}\left(\mathbb{A}^{n+1} / \mathbb{T}_{\boldsymbol{L}} \mathcal{C}_{\boldsymbol{m}, \boldsymbol{p}_{\mathbf{L}}}(\boldsymbol{X}), \boldsymbol{L}\right)=m-1$. Thus, also the intersection multiplicity of $\mathbb{P}\left(N_{\mathcal{L}_{m}(X), L}^{*}\right)$ and $\operatorname{Seg}\left(\mathbb{A}^{n+1} / L, L\right)$ at their unique point of intersection is $m-1$.

Proof of Lemma 4.29. We consider the rational map $\mathcal{L}_{m}(X) \rightarrow X, L \mapsto p_{L}$. It restricts to $\mathcal{L}_{m, p_{L}}(X) \rightarrow\left\{p_{L}\right\}$, which shows $\Phi_{L}\left(T_{\mathcal{L}_{m, p_{L}}(X), L}\right) \subset T_{\left\{p_{L}\right\}, p_{L}}=\{0\}$. Since $\mathcal{L}_{m, p_{L}}(X)$ and $\operatorname{ker} \Phi_{L}$ both have dimension $n-m$, we have derived the equality $T_{\mathcal{L}_{m, p_{L}}}(X), L=\operatorname{ker} \Phi_{L}$. The image of every $\varphi \in T_{\mathcal{L}_{m, p_{L}}(X), L}$ is contained in $\mathbb{T}_{L} \mathcal{C}_{m, p_{L}}(X) / L$ and its kernel contains $\boldsymbol{p}_{\boldsymbol{L}}$. Since both vector spaces in 4.7$)$ have the same dimension, the equality in (4.7) is proven.

For the remaining assertions, we choose coordinates $x_{0}, \ldots, x_{n}$ on $\mathbb{P}^{n}$ such 
that we can express the flag in (4.6) with the following zero loci:

$$
\begin{aligned}
p_{L} & =Z\left(x_{1}, \ldots, x_{n}\right), \\
L & =Z\left(x_{2}, \ldots, x_{n}\right), \\
\mathbb{T}_{L} \mathcal{C}_{k, p}(X) & =Z\left(x_{n-k+2}, \ldots, x_{n}\right) \quad \text { for } 2 \leq k \leq m .
\end{aligned}
$$

In the following, we work in the affine chart $\mathbb{P}^{n} \backslash Z\left(x_{0}\right) \cong \mathbb{A}^{n}$ with the standard basis $e_{1}, \ldots, e_{n}$. We extend this basis to a basis for $\mathbb{A}^{n+1}$ by adding $e_{0} \in \boldsymbol{p}_{\boldsymbol{L}} \backslash\{0\}$. Note that these coordinates are compatible with the coordinates in the proof of Theorem 4.26. We write $f(x)=f_{1}(x)+f_{2}(x)+\ldots$ for the defining equation of $X$ in the chosen affine chart, where $f_{i}$ is homogeneous of degree $i$. By our choice of coordinates, there are constants $c_{i, j}$ such that the gradients of the $f_{i}$ satisfy

$$
\begin{aligned}
\nabla f_{1} & =\left(0, \ldots, 0, c_{1, n}\right) \text { for } c_{1, n} \neq 0, \\
\nabla f_{2}\left(e_{1}\right) & =\left(0, \ldots, 0, c_{2, n-1}, c_{2, n}\right) \text { for } c_{2, n-1} \neq 0, \\
& \vdots \\
\nabla f_{m-1}\left(e_{1}\right) & =\left(0, \ldots, 0, c_{m-1, n-m+2}, \ldots, c_{m-1, n}\right) \text { for } c_{m-1, n-m+2} \neq 0 .
\end{aligned}
$$

For every tangent direction $e_{1}, \ldots, e_{n-1}$ to $X \backslash Z\left(x_{0}\right)$ at $p_{L}$, we compute its fiber under $\Phi_{L}$ as follows. For each $1 \leq i \leq n-1$, we choose a path of points $\gamma_{i}(t)=e_{i} \cdot t+O\left(t^{2}\right) \in X \backslash Z\left(x_{0}\right)$. Along the path $\gamma_{i}$, we compute all possible paths of lines $L_{i}(t) \in \mathcal{L}_{m, \gamma_{i}(t)}(X)$ such that $L_{i}(0)=L$. For this, we consider the Taylor expansion of $f$ around $\gamma_{i}(t)$. Hence, we perform a linear change of coordinates $\tilde{x}:=x-\gamma_{i}(t)$ such that $f(x)=f\left(\tilde{x}+\gamma_{i}(t)\right)=: F^{(i)}(\tilde{x})$. Now we want to write again $F^{(i)}(\tilde{x})=F_{0}^{(i)}+F_{1}^{(i)}(\tilde{x})+F_{2}^{(i)}(\tilde{x})+\ldots$, where $F_{j}^{(i)}$ is homogeneous of degree $j$ in $\tilde{x}$. For $j=1, \ldots, m$, we have $f_{j}\left(\tilde{x}+\gamma_{i}(t)\right)=$ $f_{j}(\tilde{x})+t\left\langle e_{i}, \nabla f_{j}(\tilde{x})\right\rangle+O\left(t^{2}\right)$, where $\langle\cdot, \cdot\rangle$ denotes the nondegenerate bilinear form $(x, y) \mapsto \sum_{k} x_{k} y_{k}$. This implies

$$
F_{j}^{(i)}(\tilde{x})=f_{j}(\tilde{x})+t\left\langle e_{i}, \nabla f_{j+1}(\tilde{x})\right\rangle+O\left(t^{2}\right) \quad \text { for } 1 \leq j \leq m-1
$$

The solutions of these $m-1$ polynomials are the directions with contact order of at least $m$ at $\gamma_{i}(t)$. Since we are only interested in paths of lines that start at $L$, we want to compute those solutions of $F_{1}^{(i)}(\tilde{x})=0, \ldots, F_{m-1}^{(i)}(\tilde{x})=0$ that are of the form $v(t):=e_{1}+d \cdot t+O\left(t^{2}\right)$ for some $d \in \mathbb{A}^{n}$. We get that

$$
F_{j}^{(i)}(v(t))=t\left\langle d, \nabla f_{j}\left(e_{1}\right)\right\rangle+t\left\langle e_{i}, \nabla f_{j+1}\left(e_{1}\right)\right\rangle+O\left(t^{2}\right),
$$

since $f_{j}\left(e_{1}\right)=0$ for $1 \leq j \leq m-1$. Each solution $v(t)$ of $F_{1}^{(i)}(\tilde{x})=0, \ldots$, $F_{m-1}^{(i)}(\tilde{x})=0$ defines a path of lines $L_{i, v(t)}(t) \in \mathcal{L}_{m, \gamma_{i}(t)}(X)$ starting at $L$, where each line $L_{i, v(t)}(t)$ is spanned by $\gamma_{i}(t)$ and $\gamma_{i}(t)+v(t)$. To this path of lines corresponds the tangent vector $\varphi_{i, v(t)} \in T_{\mathcal{L}_{m}(X), L} \subset \operatorname{Hom}\left(\boldsymbol{L}, \mathbb{A}^{n+1} / \boldsymbol{L}\right)$ defined 
by

$$
\begin{aligned}
& e_{0} \longmapsto \gamma_{i}^{\prime}(0)+\boldsymbol{L}=e_{i}+\boldsymbol{L}, \\
& e_{1} \longmapsto \gamma_{i}^{\prime}(0)+v^{\prime}(0)+\boldsymbol{L}=e_{i}+d+\boldsymbol{L} .
\end{aligned}
$$

Since $\varphi_{i, v(t)}$ depends only on $d$, we write $\varphi_{i, d}:=\varphi_{i, v(t)}$. The fiber of the tangent direction $e_{i}$ under $\Phi_{L}$ is spanned by all $\varphi_{i, d}$ such that $d \in \mathbb{A}^{n}$ is a solution of

$$
F_{1}^{(i)}(v(t))=0, \ldots, F_{m-1}^{(i)}(v(t))=0 .
$$

$i=1$ : The fiber of the tangent direction $e_{1}$ under $\Phi_{L}$ is $\Phi_{L}^{-1}\left(T_{L, p_{L}}\right)$. By (4.15), (4.14) and Euler's relation for homogeneous polynomials, we have

$$
\begin{aligned}
F_{1}^{(1)}(v(t)) & =c_{1, n} d_{n} \cdot t+O\left(t^{2}\right), \\
F_{2}^{(1)}(v(t)) & =\left(c_{2, n-1} d_{n-1}+c_{2, n} d_{n}\right) \cdot t+O\left(t^{2}\right), \\
\vdots & \\
F_{m-2}^{(1)}(v(t)) & =\left(c_{m-2, n-m+3} d_{n-m+3}+\ldots+c_{m-2, n} d_{n}\right) \cdot t+O\left(t^{2}\right), \\
F_{m-1}^{(1)}(v(t)) & =\left(c_{m-1, n-m+2} d_{n-m+2}+\ldots+c_{m-1, n} d_{n}+m f_{m}\left(e_{1}\right)\right) \cdot t+O\left(t^{2}\right) .
\end{aligned}
$$

Solving for $d$ implies $d_{n}=0, d_{n-1}=0, \ldots, d_{n-m+3}=0$. Since $L \in \mathcal{L}_{m}(X)$ was chosen generally, it has exactly intersection multiplicity $m$ at $p_{L}$. Thus, we have that $f_{m}\left(e_{1}\right) \neq 0$ and $d_{n-m+2}$ must be a non-zero constant. Furthermore, $d_{1}, \ldots, d_{n-m+1}$ are arbitrary. Since $\Phi_{L}^{-1}\left(T_{L, p_{L}}\right)$ is spanned by all $\varphi_{1, d}$ for these solutions $d$, where $\varphi_{1, d}$ is defined by (4.16), we have shown 4.8).

$\mathbf{2} \leq \boldsymbol{i} \leq \boldsymbol{n}-\boldsymbol{m}+\mathbf{1}$ : The fibers of the tangent directions $e_{1}, \ldots, e_{n-m+1}$ under $\Phi_{L}$ span $\Phi_{L}^{-1}\left(T_{L} \mathcal{C}_{m, p_{L}}(X)\right)$. For $1 \leq j \leq m-2$, we have $F_{j}^{(i)}(v(t))=F_{j}^{(1)}(v(t))$. This implies again $d_{n}^{(i)}=0, d_{n-1}^{(i)}=0, \ldots, d_{n-m+3}^{(i)}=0$ for a solution $d^{(i)}$ of (4.17). Furthermore, the equality

$$
F_{m-1}^{(i)}(v(t))=\left(c_{m-1, n-m+2} d_{n-m+2}+\ldots+c_{m-1, n} d_{n}+\frac{\partial f_{m}}{\partial x_{i}}\left(e_{1}\right)\right) \cdot t+O\left(t^{2}\right)
$$

shows that $d_{n-m+2}^{(i)}$ is some constant. As before, $d_{1}^{(i)}, \ldots, d_{n-m+1}^{(i)}$ are arbitrary. Since $\Phi_{L}^{-1}\left(T_{L} \mathcal{C}_{m, p_{L}}(X)\right)$ is spanned by all $\varphi_{i, d^{(i)}}$ for $1 \leq i \leq n-m+1$ and solutions $d^{(i)}$ of (4.17), where $\varphi_{i, d^{(i)}}$ is defined by (4.16), we have shown (4.9).

$\boldsymbol{i}=n-\boldsymbol{k}$ for $\mathbf{1} \leq k \leq m-2$ : For each $1 \leq l \leq m-2$, the fibers of the tangent directions $e_{1}, \ldots, e_{n-m+l+1}$ under $\Phi_{L}$ span $\Phi_{L}^{-1}\left(T_{L} \mathcal{C}_{m-l, p_{L}}(X)\right)$. For $1 \leq j \leq k-1$, we have $F_{j}^{(i)}(v(t))=F_{j}^{(1)}(v(t))$. So a solution $d^{(i)}$ of 4.17) 
satisfies $d_{n}^{(i)}=0, \ldots, d_{n-k+2}^{(i)}=0$. Since

$$
F_{k}^{(i)}(v(t))=\left(c_{k, n-k+1} d_{n-k+1}+\ldots+c_{k, n} d_{n}+c_{k+1, n-k}\right) \cdot t+O\left(t^{2}\right)
$$

and the two constants $c_{k, n-k+1}$ and $c_{k+1, n-k}$ are non-zero, we get that $d_{n-k+1}^{(i)}$ must be a non-zero constant. Finally, we have for $k+1 \leq j \leq m-1$ that

$$
F_{j}^{(i)}(v(t))=\left(c_{j, n-j+1} d_{n-j+1}+\ldots+c_{j, n} d_{n}+\frac{\partial f_{j+1}}{\partial x_{i}}\left(e_{1}\right)\right) \cdot t+O\left(t^{2}\right),
$$

which implies that $d_{n-k^{\prime}}^{(i)} \ldots, d_{n-m+2}^{(i)}$ are some constants. As before, the remaining entries $d_{1}^{(i)}, \ldots, d_{n-m+1}^{(i)}$ are arbitrary. Since $\Phi_{L}^{-1}\left(T_{L} \mathcal{C}_{m-l, p_{L}}(X)\right)$ is spanned by all $\varphi_{i, d^{(i)}}$ for $1 \leq i \leq n-m+l+1$ and solutions $d^{(i)}$ of (4.17), where $\varphi_{i, d^{(i)}}$ is defined by (4.16), we have shown 4.10 - 4.12).

Now we have classified all strongly coisotropic varieties and we have encountered non-trivial examples of coisotropic varieties. As we still do not know if coisotropy is preserved under taking singular loci, we will gain a better understanding of the singular loci of coisotropic hypersurfaces by computing them explicitly in the case of $\operatorname{Gr}\left(1, \mathbb{P}^{3}\right)$ in the next chapter. 


\section{Singular Loci of Coisotropic Hypersurfaces in $\operatorname{Gr}\left(1, \mathbb{P}^{3}\right)$}

Surfaces in $\operatorname{Gr}\left(1, \mathbb{P}^{3}\right)$ are classically known as congruences. The aim of this chapter is to study congruences which arise as singular loci of Chow hypersurfaces of space curves or of Hurwitz hypersurfaces of surfaces. We determine their classes in the Chow ring of $\operatorname{Gr}\left(1, \mathbb{P}^{3}\right)$ and their singular loci. Throughout this thesis, we use the phrase 'singular points of a congruence' to simply refer to its singularities as a subvariety of the Grassmannian $\operatorname{Gr}\left(1, \mathbb{P}^{3}\right)$. In older literature, this phrase refers to points in $\mathbb{P}^{3}$ lying on infinitely many lines of the congruence; nowadays, these are called fundamental points.

For a general point $v \in \mathbb{P}^{3}$ and a general plane $H \subset \mathbb{P}^{3}$, the bidegree of a congruence is a pair $(\alpha, \beta)$, where the order $\alpha$ is the number of points in $\Sigma$ corresponding to a line $L \subset \mathbb{P}^{3}$ such that $v \in L$ and the class $\beta$ is the number of points in $\Sigma$ corresponding to lines $L \subset \mathbb{P}^{3}$ such that $L \subset H$. For instance, consider the congruence of all lines passing through a fixed point $x$. Given a general point $v$, this congruence contains a unique line passing through $v$, namely the line spanned by $x$ and $v$. Given a general plane $H$, we have $x \notin H$, so this congruence does not contain any line that lies in $H$. Hence, the set of lines passing through a fixed point is a congruence with bidegree $(1,0)$. A similar argument shows that the congruence of lines lying in a fixed plane has bidegree $(0,1)$.

The study of congruences goes back to Kummer [69], who classified those of order one. Ran [87] studies surfaces of order one in general Grassmannians and gives a modern proof of Kummer's classification. In Section 7.3, we classify all congruences according to their strict focal loci and compare our classification to Kummer's result (see Remark 7.25). Congruences play a role in algebraic vision (e.g., see Chapter 6) and multi-view geometry, where cameras are modeled as maps from $\mathbb{P}^{3}$ to congruences [86]. The multidegree of the image of several of those cameras is computed by Escobar and Knutson in [37].

This chapter provides complete solutions to Problem 5 on Curves, Problem 4 on Surfaces, and Problem 3 on Grassmannians in [100]. Our main results are consolidated in the following theorem.

Theorem 5.1. Let $C \subset \mathbb{P}^{3}$ be a nondegenerate curve of degree $d$ and geometric genus $g$ having only ordinary singularities $x_{1}, x_{2}, \ldots, x_{s}$ with multiplicities $r_{1}, r_{2}, \ldots, r_{s}$. If $\mathrm{Sec}(C)$ denotes the locus of secant lines to $C$, then the singular locus of $\mathrm{CH}_{0}(C)$ is $\operatorname{Sec}(C) \cup \bigcup_{i=1}^{S}\left\{L \in \operatorname{Gr}\left(1, \mathbb{P}^{3}\right) \mid x_{i} \in L\right\}$, the bidegree of $\operatorname{Sec}(C)$ is

$$
\left(\frac{1}{2}(d-1)(d-2)-g-\sum_{i=1}^{s} \frac{1}{2} r_{i}\left(r_{i}-1\right), \frac{1}{2} d(d-1)\right)
$$


and the singular locus of $\operatorname{Sec}(C)$, when $C$ is smooth, consists of all lines that intersect $C$ with total multiplicity at least three.

Let $S \subset \mathbb{P}^{3}$ be a general surface of degree $d$ with $d \geq 4$. If $\operatorname{Bit}(S)$ denotes the locus of bitangents to $S$ and $\operatorname{Infl}(S)$ denotes the locus of inflectional tangents to $S$, then the singular locus of $\mathrm{CH}_{1}(S)$ is $\operatorname{Bit}(S) \cup \operatorname{Infl}(S)$, the bidegree of $\operatorname{Bit}(S)$ is

$$
\left(\frac{1}{2} d(d-1)(d-2)(d-3), \frac{1}{2} d(d-2)(d-3)(d+3)\right),
$$

and the bidegree of $\operatorname{Infl}(S)$ is $(d(d-1)(d-2), 3 d(d-2))$. Moreover, the singular locus of $\operatorname{Infl}(S)$ consists of all lines that are inflectional tangents at at least two points of $S$ or intersect $S$ with multiplicity at least four at some point.

The bidegree of $\operatorname{Infl}(S)$ also appears in [77, Prop. 4.1]. The bidegrees of $\operatorname{Bit}(S)$, $\operatorname{Infl}(S)$, and $\operatorname{Sec}(C)$, for smooth $C$, already appear in [5]. Nevertheless, we give new, more geometric, proofs not relying on Chern class techniques. The singular loci of $\operatorname{Sec}(C), \operatorname{Bit}(S)$, and $\operatorname{Infl}(S)$ are partially described in Lemma 2.3, Lemma 4.3, and Lemma 4.6 in [5].

Using duality, we establish some relationships of the varieties in Theorem 5.1 .

Theorem 5.2. If $C$ is a smooth nondegenerate irreducible space curve, then the secant lines of $C$ are dual to the bitangent lines of the dual surface $C^{\vee}$ and the tangent lines of $C$ are dual to the inflectional tangent lines of $C^{\vee}$.

If $S \subset \mathbb{P}^{3}$ is an irreducible surface with dual surface $S^{\vee}$, then the bitangent lines of $S$ and $S^{\vee}$ are dual to each other.

We show the duality of the inflectional tangents of a surface and its dual surface in Corollary 7.31.

Section 5.1 studies the singular locus of the Chow hypersurface of a space curve and computes its bidegree. Section 5.2 describes the singular locus of a Hurwitz hypersurface and Section 5.3 uses projective duality to calculate the bidegree of its components. In Section 5.4, we connect the intersection theory in $\operatorname{Gr}\left(1, \mathbb{P}^{3}\right)$ to Chow and Hurwitz hypersurfaces. Section 5.5 analyzes the singular loci of secant, bitangent, and inflectional congruences. Finally, in Section 5.6, we compute tangent spaces at smooth points of these congruences. An application to algebraic vision of these computations can be found in Chapter 6, where we determine the irreducible components of the visual event surface of a given curve or surface.

First we record a technical lemma which will be useful throughout the rest of this chapter.

Lemma 5.3. Let $f: X \rightarrow Y$ be a birational finite surjective morphism between irreducible projective varieties and let $y \in Y$. The variety $Y$ is smooth at the point $y$ if and only if the fiber $f^{-1}(y)$ contains exactly one point $x \in X$, the variety $X$ is smooth at the point $x$, and the differential $D_{x} f: T_{X, x} \rightarrow T_{Y, y}$ is an injection.

We will prove this with the help of Zariski's Main and Connectedness Theorems as well as criteria for finite maps and isomorphisms. 
Theorem 5.4 (Zariski's Main Theorem). Let $f: X \rightarrow Y$ be a birational morphism with finite fibers between irreducible varieties. If $Y$ is normal, then $f$ is an isomorphism of $X$ with an open subset of $Y$.

Theorem 5.5 (Zariski's Connectedness Theorem). Let $f: X \rightarrow Y$ be a birational morphism between irreducible projective varieties and let $y \in Y$. If $Y$ is normal at $y$, then $f^{-1}(y)$ is a connected set in the Zariski topology.

Lemma 5.6 (Criterion for Finite Maps). Let $f: X \rightarrow Y$ be a morphism between irreducible projective varieties. Let $Y_{0} \subset Y$ be open, $X_{0}:=f^{-1}\left(Y_{0}\right)$, and let $f_{0}$ be the restriction of $f$ to $X_{0}$. The morphism $f_{0}$ is finite if and only if its fibers are finite.

Theorem 5.7 (Criterion for Isomorphisms). Let $f: X \rightarrow Y$ be a finite morphism between irreducible varieties. The map $f$ is an isomorphism if and only if it is bijective and the differential $D_{x} f: T_{X, x} \rightarrow T_{Y, f(y)}$ is an injection for all $x \in X$.

The above formulation of Zariski's Main and Connectedness Theorems can for example be found in [75, Ch. III.9, p. 288-289, Thm. I, Thm. V], whereas the two criteria appear in [49, Lem. 14.8, Thm 14.9].

Proof of Lemma 5.3 First, suppose that $Y$ is smooth at the point $y$. Since $Y$ is normal at the point $y$, Zariski's Connectedness Theorem proves that the fiber $f^{-1}(y)$ is a connected set in the Zariski topology. As $f$ is a finite morphism, its fibers are finite and we deduce that $f^{-1}(y)=\{x\}$. For $X_{0}:=f^{-1}(\operatorname{Reg}(Y))$, Zariski's Main Theorem implies that the restriction of $f$ to $X_{0}$ is an isomorphism of $X_{0}$ with $\operatorname{Reg}(Y)$. In particular, we have that $x \in X_{0} \subset X$ is a smooth point. Moreover, the above criterion for isomorphisms shows that the differential $D_{x} f$ is injective.

For the other direction, suppose that $f^{-1}(y)=\{x\}$ for some smooth point $x \in X$ with injective differential $D_{x} f$. Let $Y_{1}$ be an open neighborhood of $y$ containing points in $Y$ with one-element fibers and injective differentials. Combining the two criteria in Lemma 5.6 and Theorem 5.7produces an isomorphism of $X_{1}:=f^{-1}\left(Y_{1}\right)$ with $Y_{1}$. Since $x \in X_{1}$ is smooth, we conclude that $y \in Y_{1} \subset Y$ is smooth.

\subsection{Secants of Space Curves}

This section describes the singular locus of the Chow hypersurface of a curve $C \subset \mathbb{P}^{3}$. For a curve with mild singularities, we also compute the bidegree of this locus.

If $C$ has degree at least two, the set of lines that meet it in two points forms a surface $\operatorname{Sec}(C) \subset \operatorname{Gr}\left(1, \mathbb{P}^{3}\right)$ called the secant congruence of $C$. More precisely, $\operatorname{Sec}(C)$ is the Zariski closure in $\operatorname{Gr}\left(1, \mathbb{P}^{3}\right)$ of the set of points corresponding to a line in $\mathbb{P}^{3}$ which intersects the curve $C$ at two smooth points. In particular, $\operatorname{Sec}(C)$ contains the tangent lines at smooth points of $C$. A line meeting $C$ at a singular point might not belong to $\operatorname{Sec}(C)$, even though it has intersection multiplicity at least two with the curve; see Remark 5.9. 
The following theorem is the main result in this section.

Theorem 5.8. Let $C \subset \mathbb{P}^{3}$ be an irreducible curve of degree at least two. The singular locus of the Chow hypersurface of $C$ is

$$
\operatorname{Sec}(C) \cup\left(\bigcup_{x \in \operatorname{Sing}(C)}\left\{L \in \operatorname{Gr}\left(1, \mathbb{P}^{3}\right) \mid x \in L\right\}\right)
$$

Proof. We first show that the incidence variety

$$
\Phi_{C}:=\{(v, L) \mid v \in L\} \subset C \times \operatorname{Gr}\left(1, \mathbb{P}^{3}\right)
$$

is smooth at the point $(v, L)$ if and only if the curve $C$ is smooth at the point $v \in C$. Let $f_{1}, f_{2}, \ldots, f_{k}$ be generators for the vanishing ideal of $C$ in the unknowns $x_{0}, \ldots, x_{3}$. We use dual Plücker coordinates $q_{01}, \ldots, q_{23}$ on $\operatorname{Gr}\left(1, \mathbb{P}^{3}\right)$. Consider the affine chart of $\mathbb{P}^{3} \times \operatorname{Gr}\left(1, \mathbb{P}^{3}\right)$ where $x_{0} \neq 0$ and $q_{01} \neq 0$. We may assume that $v=(1: \alpha: \beta: \gamma)$ and the line $L$ is spanned by the points $(1: 0: a: b)$ and $(0: 1: c: d)$. We have that $v \in L$ if and only if the line $L$ is given by the row space of the matrix

$$
\left[\begin{array}{cccc}
1 & \alpha & \beta & \gamma \\
0 & 1 & c & d
\end{array}\right]=\left[\begin{array}{ll}
1 & \alpha \\
0 & 1
\end{array}\right]\left[\begin{array}{cccc}
1 & 0 & \beta-\alpha c & \gamma-\alpha d \\
0 & 1 & c & d
\end{array}\right]
$$

which is equivalent to $a=\beta-\alpha c$ and $b=\gamma-\alpha d$. Hence, in the chosen affine chart, $\Phi_{C}$ can be written as

$$
\left\{(\alpha, \beta, \gamma, a, b, c, d) \mid f_{i}(1, \alpha, \beta, \gamma)=0 \text { for } 1 \leq i \leq k, a=\beta-\alpha c, b=\gamma-\alpha d\right\} \text {. }
$$

As $\operatorname{dim} \Phi_{C}=3$, it is smooth at the point $(v, L)$ if and only if its tangent space has dimension three or, equivalently, the Jacobian matrix

$$
\left[\begin{array}{ccccccc}
\frac{\partial f_{1}}{\partial x_{1}}(1, \alpha, \beta, \gamma) & \frac{\partial f_{1}}{\partial x_{2}}(1, \alpha, \beta, \gamma) & \frac{\partial f_{1}}{\partial x_{3}}(1, \alpha, \beta, \gamma) & 0 & 0 & 0 & 0 \\
\frac{\partial f_{2}}{\partial x_{1}}(1, \alpha, \beta, \gamma) & \frac{\partial f_{2}}{\partial x_{2}}(1, \alpha, \beta, \gamma) & \frac{\partial f_{2}}{\partial x_{3}}(1, \alpha, \beta, \gamma) & 0 & 0 & 0 & 0 \\
\vdots & \vdots & \vdots & \vdots & \vdots & \vdots & \vdots \\
\frac{\partial f_{k}}{\partial x_{1}}(1, \alpha, \beta, \gamma) & \frac{\partial f_{k}}{\partial x_{2}}(1, \alpha, \beta, \gamma) & \frac{\partial f_{k}}{\partial x_{3}}(1, \alpha, \beta, \gamma) & 0 & 0 & 0 & 0 \\
-c & 1 & 0 & -1 & 0 & -\alpha & 0 \\
-d & 0 & 1 & 0 & -1 & 0 & -\alpha
\end{array}\right]
$$

has rank four. We see that this Jacobian matrix has rank four if and only if the Jacobian matrix of $C$ has rank two, in which case $v \in C$ is smooth. Therefore, we deduce that $\Phi_{C}$ is smooth at the point $(v, L)$ exactly when $C$ is smooth at the point $v$.

By Lemma 5.6, the projection $\pi: \Phi_{C} \rightarrow \mathrm{CH}_{0}(C)$ defined by $(v, L) \mapsto L$ is finite; otherwise $C$ would contain a line contradicting our assumptions. Moreover, the general fiber of $\pi$ has cardinality one because the general line 
$L \in \mathrm{CH}_{0}(C)$ intersects $C$ in a single point. Hence, $\pi$ is birational. Applying Lemma 5.3 shows that $\mathrm{CH}_{0}(C)$ is smooth at $L$ if and only if $\pi^{-1}(L)=\{(v, L)\}$ where $v \in C$ is a smooth point and the differential $D_{(v, L)} \pi$ is injective. Using our chosen affine chart, we see that the differential $D_{(v, L)} \pi$ sends every element in the kernel of the Jacobian matrix to its last four coordinates. This map is not injective if and only if the kernel contains an element of the form $(*, *, *, 0,0,0,0)^{T} \neq 0$. Such an element belongs to the kernel if and only if it is $(\lambda, c \lambda, d \lambda, 0,0,0,0)^{T}$ for some non-zero constant $\lambda$ and

$$
\frac{\partial f_{i}}{\partial x_{1}}(1, \alpha, \beta, \gamma)+c \frac{\partial f_{i}}{\partial x_{2}}(1, \alpha, \beta, \gamma)+d \frac{\partial f_{i}}{\partial x_{3}}(1, \alpha, \beta, \gamma)=0
$$

for all $1 \leq i \leq k$. Hence, for a smooth point $v \in C$, the differential $D_{(v, L)} \pi$ is not injective if and only if $L$ is the tangent line of $C$ at $v$. Since we have that all tangent lines to $C$ are contained in $\operatorname{Sec}(C)$ and that $\left|\pi^{-1}(L)\right|=1$ if and only if $L$ is not a secant line, we conclude that $\mathrm{CH}_{0}(C)$ is smooth at $L$ if and only if $L \notin \operatorname{Sec}(C)$ and $L$ meets $C$ at a smooth point.

Remark 5.9. Local computations show that the secant congruence of $C$ generally does not contain all lines through singular points of $C$. To be more explicit, let $x \in C$ be an ordinary singularity; the point $x$ is the intersection of $r$ branches of $C$ with $r \geq 2$, and the $r$ tangent lines of the branches at $x$ are pairwise different. We claim that a line $L$ intersecting $C$ only at the point $x$ is contained in $\operatorname{Sec}(C)$ if and only if $L$ lies in a plane spanned by two of the $r$ tangent lines at $x$. The union of all those lines forms the tangent star of $C$ at $x$; see [57, 98].

Suppose that $x=(1: 0: 0: 0)$ and $L \in \operatorname{Sec}(C)$ intersects the curve $C$ only at the point $x$. The line $L$ must be the limit of a family of lines $L_{t}$ that intersect $C$ at two distinct smooth points. Without loss of generality, the line $L$ is not one of the tangent lines of the curve $C$ at the point $x$ and each line $L_{t}$ intersects at least two distinct branches of $C$. Since there are only finitely many branches, we can also assume that each line $L_{t}$ in the family intersects the same two branches of the curve $C$. These two branches are parametrized by $\left(1: f_{1}(s): f_{2}(s): f_{3}(s)\right)$ and $\left(1: g_{1}(s): g_{2}(s): g_{3}(s)\right)$ with $f_{i}(0)=0=g_{j}(0)$ for $1 \leq i, j \leq 3$. Thus, the tangent lines to these branches are spanned by $x$ and $\left(1: f_{1}^{\prime}(0): f_{2}^{\prime}(0): f_{3}^{\prime}(0)\right)$ or $\left(1: g_{1}^{\prime}(0): g_{2}^{\prime}(0): g_{3}^{\prime}(0)\right)$. Parametrizing intersection points, we see that the line $L_{t}$ intersects the first branch at $\left(1: f_{1}(\varphi(t)): f_{2}(\varphi(t)): f_{3}(\varphi(t))\right)$ and the second branch at $\left(1: g_{1}(\psi(t)): g_{2}(\psi(t)): g_{3}(\psi(t))\right)$ where $\varphi(0)=0=\psi(0)$. Hence, the dual Plücker coordinates for $L_{t}$ are

$$
\left(\frac{g_{1}(\psi(t))-f_{1}(\varphi(t))}{t}: \frac{g_{2}(\psi(t))-f_{2}(\varphi(t))}{t}: \cdots: \frac{f_{2}(\varphi(t)) g_{3}(\psi(t))-f_{3}(\varphi(t)) g_{2}(\psi(t))}{t}\right) .
$$

Taking the limit as $t \rightarrow 0$, we obtain the line $L$ with Plücker coordinates

$$
\left(g_{1}^{\prime}(0) \psi^{\prime}(0)-f_{1}^{\prime}(0) \varphi^{\prime}(0): \ldots: g_{3}^{\prime}(0) \psi^{\prime}(0)-f_{3}^{\prime}(0) \varphi^{\prime}(0): 0: 0: 0\right) \text {. }
$$


This line is spanned by the point $x$ and

$$
\left(1: g_{1}^{\prime}(0) \psi^{\prime}(0)-f_{1}^{\prime}(0) \varphi^{\prime}(0): g_{2}^{\prime}(0) \psi^{\prime}(0)-f_{2}^{\prime}(0) \varphi^{\prime}(0): g_{3}^{\prime}(0) \psi^{\prime}(0)-f_{3}^{\prime}(0) \varphi^{\prime}(0)\right),
$$

so it lies in the plane spanned by the two tangent lines. From this computation, we also see that all lines passing through $x$ and lying in the plane spanned by the tangent lines can be approximated by lines that intersect both of the branches at points different from $x$. For this, one need only choose $\varphi(t)=\lambda t$ and $\psi(t)=\mu t$ for all possible non-zero constants $\lambda$ and $\mu$.

Using Chern classes, Proposition 2.1 in [5] calculates the bidegree of the secant congruence of a smooth curve. We give a geometric description of this bidegree and extend it to curves with ordinary singularities.

Theorem 5.10. If $C \subset \mathbb{P}^{3}$ is a nondegenerate irreducible curve of degree $d$ and genus $g$ having only ordinary singularities $x_{1}, x_{2}, \ldots, x_{s}$ with multiplicities $r_{1}, r_{2}, \ldots, r_{s}$, then the bidegree of the secant congruence $\operatorname{Sec}(C)$ is

$$
\left(\left(\begin{array}{c}
d-1 \\
2
\end{array}\right)-g-\sum_{i=1}^{s}\left(\begin{array}{l}
r_{i} \\
2
\end{array}\right),\left(\begin{array}{l}
d \\
2
\end{array}\right)\right)
$$

Proof. Let $H \subset \mathbb{P}^{3}$ be a general plane. The intersection of $H$ with $C$ consists of $d$ points. Any two of these points define a secant line lying in $H$; see Fig. 5.1. Hence, there are $\left(\begin{array}{l}d \\ 2\end{array}\right)$ secant lines contained in $H$, which gives the class of $\operatorname{Sec}(C)$.

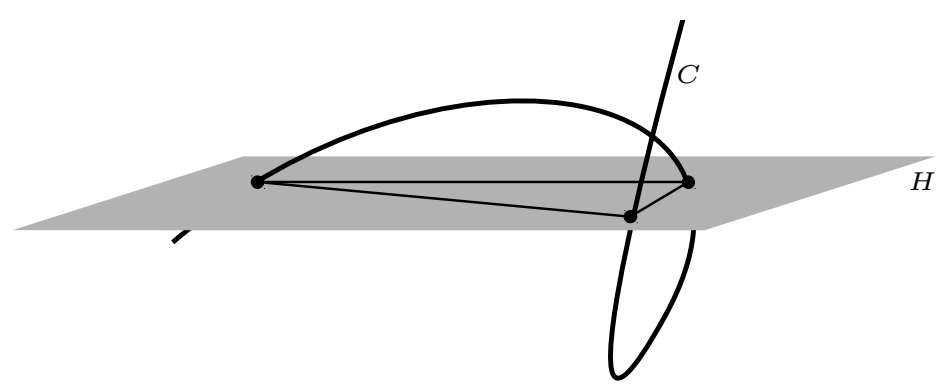

Figure 5.1: The class of the secant congruence of a space curve.

To compute the order of $\operatorname{Sec}(C)$, let $v \in \mathbb{P}^{3}$ be a general point. Projecting away from $v$ defines a rational map $\pi_{v}: \mathbb{P}^{3} \rightarrow \mathbb{P}^{2}$. Set $C^{\prime}:=\pi_{v}(C)$. The map $\pi_{v}$ sends a line passing through $v$ and intersecting $C$ at two points to a simple node of the plane curve $C^{\prime}$; see Fig. 5.5. Moreover, every ordinary singularity of $C$ is sent to an ordinary singularity of $C^{\prime}$ with the same multiplicity, and the plane curve $C^{\prime}$ has the same degree as the space curve $C$. As the geometric genus is invariant under birational transformations, it also has the same genus; see [50, Thm. II.8.19]. Thus, the genus-degree formula for plane curves [93, p. 54, Eq. (7)] shows that the genus of $C$ is equal to $\left(\begin{array}{c}d-1 \\ 2\end{array}\right)-\sum_{i=1}^{S}\left(\begin{array}{c}r_{i} \\ 2\end{array}\right)$ minus the number of secants of $C$ passing through $v$. 
Remark 5.11. If a curve $C \subset \mathbb{P}^{3}$ of degree at least two is contained in a plane, its secant congruence consists of all lines in that plane and has bidegree $(0,1)$.

Problem 5 on Curves in [100] asks to compute the dimension and bidegree of $\operatorname{Sing}\left(\mathrm{CH}_{0}(C)\right)$. When $C$ is not a line, Theorem 5.8 establishes that $\operatorname{Sing}\left(\mathrm{CH}_{0}(C)\right)$ is two-dimensional. For completeness, we also state its bidegree explicitly.

Corollary 5.12. If $C \subset \mathbb{P}^{3}$ is an irreducible curve of degree $d \geq 2$ and geometric genus $g$ having only ordinary singularities $x_{1}, x_{2}, \ldots, x_{s}$ with multiplicities $r_{1}, r_{2}, \ldots, r_{s}$, the bidegree of Sing $\left(\mathrm{CH}_{0}(C)\right)$ equals $\left(\left(\begin{array}{c}d-1 \\ 2\end{array}\right)-g-\sum_{i=1}^{s}\left(\begin{array}{c}r_{i} \\ 2\end{array}\right)+s,\left(\begin{array}{l}d \\ 2\end{array}\right)\right)$ if $C$ is nondegenerate, and $(s, 1)$ if $C$ is contained in a plane.

Proof. The bidegree of each congruence $\left\{L \in \operatorname{Gr}\left(1, \mathbb{P}^{3}\right) \mid x_{i} \in L\right\}$ is $(1,0)$. Hence, combining Theorem 5.8, Theorem 5.10, and Remark 5.11 proves the corollary.

\subsection{Bitangents and Inflections of Hypersurfaces}

This section describes the singular locus of the Hurwitz hypersurface of a hypersurface in $\mathbb{P}^{n}$. Although the main interest of this chapter lies on subvarieties of $\operatorname{Gr}\left(1, \mathbb{P}^{3}\right)$, we study Hurwitz hypersurfaces of $\operatorname{Gr}\left(1, \mathbb{P}^{n}\right)$ in this section, since the proof of Theorem 5.13 for $n=3$ generalizes immediately to higher dimensions as it is given below.

In analogy with the secant congruence of a curve, we associate two congruences to a surface $S \subset \mathbb{P}^{3}$. Specifically, the Zariski closure in $\operatorname{Gr}\left(1, \mathbb{P}^{3}\right)$ of the set of lines which are tangent to a surface $S$ at two smooth points forms the bitangent locus $\operatorname{Bit}(S)$. The inflectional locus $\operatorname{Infl}(S):=\mathcal{L}_{3}(S)$ associated to $S$ is the Zariski closure in $\operatorname{Gr}\left(1, \mathbb{P}^{3}\right)$ of the set of lines that intersect the surface $S$ at a smooth point with multiplicity at least three. For a general surface $S$, the bitangent and inflectional loci are congruences. However, this is not always the case, as Remarks 5.21 and 5.23 demonstrate.

More generally, for a hypersurface $X \subset \mathbb{P}^{n}$, we set $\operatorname{Infl}(X):=\mathcal{L}_{3}(X)$ and denote by $\operatorname{Bit}(X) \subset \operatorname{Gr}\left(1, \mathbb{P}^{n}\right)$ the Zariski closure of the set of lines which are tangent to $X$ at two smooth points. In parallel with Section 5.1, the main result in this section describes the singular locus of the Hurwitz hypersurface of $X$.

Theorem 5.13. If $n \geq 3$ and $X \subset \mathbb{P}^{n}$ is an irreducible smooth hypersurface of degree at least $2(n-1)$ which does not contain any lines, then we have

$$
\operatorname{Sing}\left(\mathrm{CH}_{1}(X)\right)=\operatorname{Bit}(X) \cup \operatorname{Infl}(X) \text {. }
$$

Proof. We first show that the incidence variety

$$
\Phi_{X}:=\left\{(v, L) \mid v \in L \subset \mathbb{T}_{X, v}\right\} \subset X \times \operatorname{Gr}\left(1, \mathbb{P}^{n}\right)
$$

is smooth. Let $f$ be the defining equation for $X$ in $\mathbb{P}^{n}$ in the unknowns $x_{0}, \ldots, x_{n}$. We use dual Plücker coordinates $q_{01}, q_{02}, \ldots$ on $\operatorname{Gr}\left(1, \mathbb{P}^{n}\right)$. Consider the affine 
chart in $\mathbb{P}^{n} \times \operatorname{Gr}\left(1, \mathbb{P}^{n}\right)$ where $x_{0} \neq 0$ and $q_{01} \neq 0$. We may assume that $v=$ $\left(1: \alpha_{1}: \ldots: \alpha_{n}\right)$ and the line $L$ is spanned by the points $\left(1: 0: b_{2}: \ldots: b_{n}\right)$ and $\left(0: 1: c_{2}: \ldots: c_{n}\right)$. In this affine chart, $X$ is defined by $g_{0}\left(x_{1}, \ldots, x_{n}\right):=$ $f\left(1, x_{1}, \ldots, x_{n}\right)$. As in the proof of Theorem 5.8, we have that $v \in L$ if and only if $b_{j}=\alpha_{j}-\alpha_{1} c_{j}$ for $2 \leq j \leq n$. For such a pair $(v, L)$, we also have that $L \subset \mathbb{T}_{X, v}$ if and only if $\left(0: 1: c_{2}: \ldots: c_{n}\right) \in \mathbb{T}_{X, v}$. Setting $g_{1}:=\frac{\partial g_{0}}{\partial x_{1}}+c_{2} \frac{\partial g_{0}}{\partial x_{2}}+\ldots+c_{n} \frac{\partial g_{0}}{\partial x_{n}}$, we have $L \subset \mathbb{T}_{X, v}$ if and only if $g_{1}\left(\alpha_{1}, \ldots, \alpha_{n}\right)=0$. Hence, in the chosen affine chart, $\Phi_{X}$ can be written as

$$
\left\{\begin{array}{l|l}
\left(\alpha_{1}, \ldots, \alpha_{n}, b_{2}, \ldots, b_{n}, c_{2}, \ldots, c_{n}\right) & \begin{array}{l}
g_{i}\left(\alpha_{1}, \ldots, \alpha_{n}\right)=0 \text { for } 0 \leq i \leq 1 \\
b_{j}=\alpha_{j}-\alpha_{1} c_{j} \text { for } 2 \leq j \leq n
\end{array}
\end{array}\right\}
$$

As $\operatorname{dim} \Phi_{X}=2 n-3$, it is smooth at the point $(v, L)$ if and only if its tangent space has dimension $2 n-3$ or, equivalently, its Jacobian matrix

$$
\left[\begin{array}{cccccccccc}
\frac{\partial g_{0}}{\partial x_{1}}(\alpha) & \frac{\partial g_{0}}{\partial x_{2}}(\alpha) & \cdots & \frac{\partial g_{0}}{\partial x_{n}}(\alpha) & 0 & \cdots & 0 & 0 & \cdots & 0 \\
\frac{\partial g_{1}}{\partial x_{1}}(\alpha) & \frac{\partial g_{1}}{\partial x_{2}}(\alpha) & \cdots & \frac{\partial g_{1}}{\partial x_{n}}(\alpha) & 0 & \cdots & 0 & \frac{\partial g_{0}}{\partial x_{2}}(\alpha) & \cdots & \frac{\partial g_{0}}{\partial x_{n}}(\alpha) \\
-c_{2} & 1 & & 0 & -1 & & 0 & -\alpha_{1} & & 0 \\
\vdots & & \ddots & & & \ddots & & & \ddots & \\
-c_{n} & 0 & & 1 & 0 & & -1 & 0 & & -\alpha_{1}
\end{array}\right]
$$

has full rank $n+1$. Since $X$ is smooth, we deduce that this Jacobian matrix has full rank, so $\Phi_{X}$ is also smooth.

Since $X$ does not contain lines, all fibers of the projection $\pi: \Phi_{X} \rightarrow \mathrm{CH}_{1}(X)$ defined by $(v, L) \mapsto L$ are finite, so Lemma 5.6 implies that $\pi$ is finite. Moreover, the general fiber of $\pi$ has cardinality one, so $\pi$ is birational. Applying Lemma 5.3 shows that $\mathrm{CH}_{1}(X)$ is smooth at the point $L$ if and only if the fiber $\pi^{-1}(L)$ consists of one point $(v, L)$ and the differential $D_{(v, L)} \pi$ is injective. In particular, we have $\left|\pi^{-1}(L)\right|=1$ if and only if $L$ is not a bitangent. It remains to show that the differential $D_{(v, L)} \pi$ is injective if and only if $L$ is a simple tangent of $X$ at $v$. Using our chosen affine chart, we see that the differential $D_{(v, L)} \pi$ projects every element in the kernel of the Jacobian matrix on its last $2(n-1)$ coordinates. This map is not injective if and only if the kernel contains an element of the form $(*, \ldots, *, 0, \ldots, 0)^{T} \neq 0$. Such an element belongs to the kernel if and only if it is equal to $\left(\lambda, c_{2} \lambda, \ldots, c_{n} \lambda, 0, \ldots, 0\right)^{T}$ for some non-zero constant $\lambda$ and $g_{1}(\alpha)=0=g_{2}(\alpha)$ where $g_{2}:=\frac{\partial g_{1}}{\partial x_{1}}+c_{2} \frac{\partial g_{1}}{\partial x_{2}}+\ldots+c_{n} \frac{\partial g_{1}}{\partial x_{n}}$. Parametrizing the line $L$ by

$$
\ell(s, t):=\left(s: s \alpha_{1}+t: s \alpha_{2}+t c_{2}: \ldots: s \alpha_{n}+t c_{n}\right)
$$

for $(s: t) \in \mathbb{P}^{1}$ shows that the line $L$ intersects the hypersurface $X$ with multiplicity at least three at $v$ if and only if $f(\ell(s, t))$ is divisible by $t^{3}$. This is equivalent to the conditions that $g_{1}(\alpha)=\left.\frac{\partial}{\partial t}[f(\ell(s, t))]\right|_{(1,0)}=0$ and $g_{2}(\alpha)=$ $\left.\frac{\partial^{2}}{\partial^{2} t}[f(\ell(s, t))]\right|_{(1,0)}=0$. 
Remark 5.14. When the degree of $X$ is at least $2(n-1)$, the hypothesis that $X$ does not contain any lines is relatively mild, since a general hypersurface of degree at least $2(n-1)$ in $\mathbb{P}^{n}$ does not contain a line; see [105].

We can generalize Theorem 5.13 to hypersurfaces in $\mathbb{P}^{n}$ which contain at most finitely many lines and have degree at least $2 n-3$ (note that general hypersurfaces of degree $2 n-3$ contain finitely many lines [105]): for an irreducible smooth hypersurface $X \subset \mathbb{P}^{n}$ with $\operatorname{deg} X \geq 2 n-3$ which contains finitely many lines $L_{1}, \ldots, L_{k}$, we have

$$
\operatorname{Bit}(X) \cup \operatorname{Infl}(X) \subset \operatorname{Sing}\left(\mathrm{CH}_{1}(X)\right) \subset \operatorname{Bit}(X) \cup \operatorname{Infl}(X) \cup\left\{L_{1}, \ldots, L_{k}\right\} .
$$

In the proof of Theorem 5.13, we have used the assumption that $X$ contains no lines only to see that the projection $\pi: \Phi_{X} \rightarrow \mathrm{CH}_{1}(X)$ is finite. Hence, the proof of (5.1) is completely analogous to the proof of Theorem 5.13, with the only exception that we have to define the incidence variety $\Phi_{X}$ as follows:

$$
\Phi_{X}:=\left\{(v, L) \mid v \in L \subset \mathbb{T}_{X, v}, L \notin\left\{L_{1}, \ldots, L_{k}\right\}\right\} \subset X \times \operatorname{Gr}\left(1, \mathbb{P}^{n}\right) .
$$

\subsection{Projective Duality}

This section uses projective duality to compute the bidegrees of the components of the singular locus of the Hurwitz hypersurface of a surface in $\mathbb{P}^{3}$, and to relate the secant, bitangent and inflectional congruences of projectively dual curves and surfaces.

Plücker's formula, which relates the number and type of singularities of a plane curve to the degree of its dual curve, plays an important role in calculating the bidegrees of the bitangent and inflectional congruences. A point $v$ on a planar curve $C$ is a simple node or a cusp if the formal completion of $\mathcal{O}_{C, v}$ is isomorphic to $\mathbb{C} \llbracket z_{1}, z_{2} \rrbracket /\left(z_{1}^{2}+z_{2}^{2}\right)$ or $\mathbb{C} \llbracket z_{1}, z_{2} \rrbracket /\left(z_{1}^{3}+z_{2}^{2}\right)$ respectively; see Fig. 5.2 . Both singularities have multiplicity two; nodes have two distinct tangents and cusps have a single tangent.

Lemma 5.15 (Plücker's formula [32, Example 1.2.8]). If $C \subset \mathbb{P}^{2}$ is an irreducible curve of degree $d$ with exactly $\kappa$ cusps, $\delta$ simple nodes, and no other singularities, then the degree of the dual curve $C^{\vee}$ is $d(d-1)-3 \kappa-2 \delta$.

Sketch of proof. Let $f$ be the defining equation for $C$ in $\mathbb{P}^{2}$ in the unknowns $x_{0}, x_{1}, x_{2}$, so we have $\operatorname{deg}(f)=d$. To begin, assume that $C$ is smooth. The degree of its dual $C^{\vee} \subset\left(\mathbb{P}^{2}\right)^{*}$ is the number of points of $C^{\vee}$ lying on a general line $L \subset\left(\mathbb{P}^{2}\right)^{*}$. By duality, the degree equals the number of tangent lines to $C$ passing through a general point $y \in \mathbb{P}^{2}$. Such a tangent line at the point $v \in C$ passes through the point $y$ if and only if $g:=y_{0} \frac{\partial f}{\partial x_{0}}(v)+y_{1} \frac{\partial f}{\partial x_{1}}(v)+y_{2} \frac{\partial f}{\partial x_{2}}(v)=0$. Hence, the degree of $C^{\vee}$ is the number of points in the zero locus $Z(f, g)$. Since $\operatorname{deg}(g)=d-1$, this finite set contains $d(d-1)$ points.

If $C$ is singular, then the degree of $C^{\vee}$ is the number of lines that are tangent to $C$ at a smooth point and pass through the general point $y$. Those smooth points 

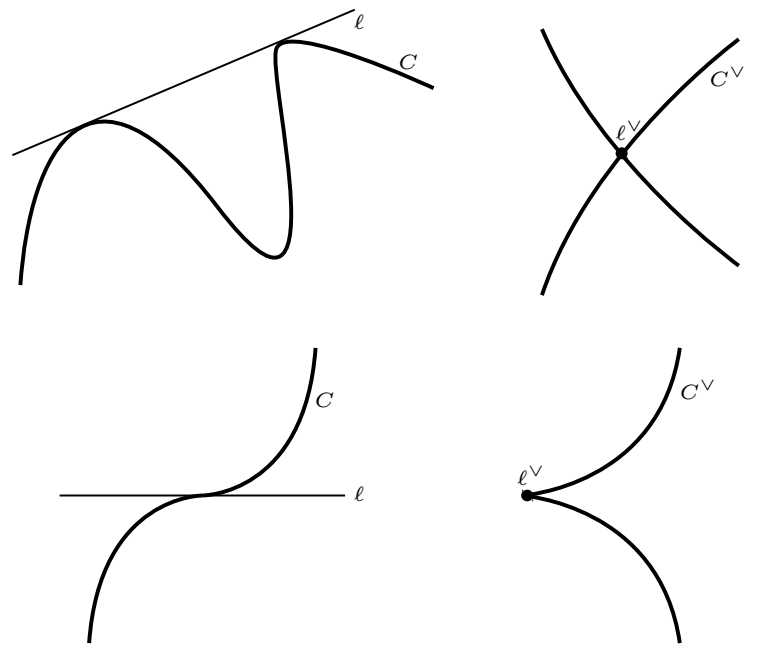

Figure 5.2: A bitangent and an inflectional line corresponding to a node and a cusp of the dual curve.

are contained in the set $Z(f, g)$, but all of the singular points also lie in $Z(f, g)$. The curve $Z(g)$ passes through each node of $C$ with intersection multiplicity two and through each cusp of $C$ with intersection multiplicity three. Therefore, we conclude that $\operatorname{deg}\left(C^{\vee}\right)=d(d-1)-3 \kappa-2 \delta$.

Using Lemma 5.15, we can compute the degree of the Hurwitz hypersurface of a smooth surface; this formula also follows from Theorem 1.1 in [101].

Proposition 5.16. For an irreducible smooth surface $S \subset \mathbb{P}^{3}$ of degree $d$ with $d \geq 2$, the degree of the Hurwitz hypersurface $\mathrm{CH}_{1}(S)$ is $d(d-1)$.

Proof. Let $H \subset \mathbb{P}^{3}$ be a general plane and $v \in H$ be a general point. The degree of $\mathrm{CH}_{1}(S)$ is the number of tangent lines $L$ to $S$ such that $v \in L \subset H$. Bertini's Theorem [49, Thm. 17.16] implies that the intersection $S \cap H$ is a smooth plane curve of degree $d$. The degree of $\mathrm{CH}_{1}(S)$ is the number of tangent lines to $S \cap H$ passing through the general point $v$; see Fig. 5.3. By definition, this is equal to the degree of the dual plane curve $(S \cap H)^{\nabla}$, so Lemma 5.15 shows $\operatorname{deg}\left(\mathrm{CH}_{1}(S)\right)=d(d-1)$.

Using Lemma 5.15, we can also count the number of bitangents and inflectional tangents to a general smooth plane curve.

Proposition 5.17. A general smooth irreducible curve in $\mathbb{P}^{2}$ of degree d has exactly $\frac{1}{2} d(d-2)(d-3)(d+3)$ bitangents and $3 d(d-2)$ inflectional tangents.

Proof. Let $C \subset \mathbb{P}^{2}$ be a general smooth irreducible curve of degree $d$. A bitangent to $C$ corresponds to a node of $C^{\vee}$, and an inflectional tangent to $C$ corresponds to a cusp of $C^{\vee}$; see Fig. 5.2 and [47, pp. 277-278]. Lemma 5.15 shows 


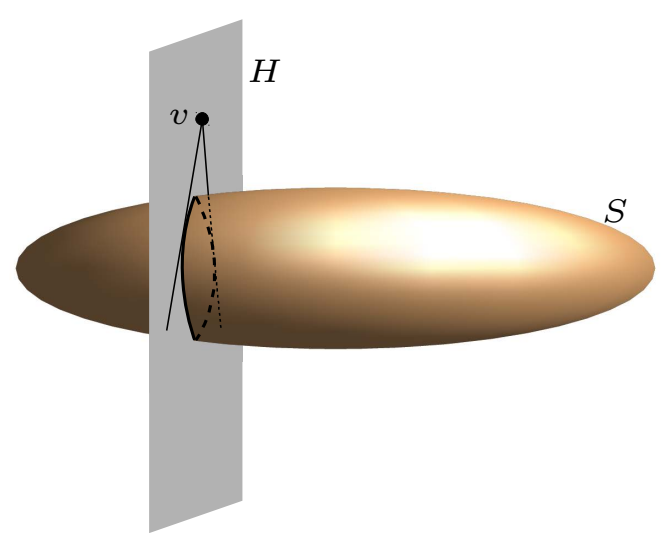

Figure 5.3: The degree of the Hurwitz hypersurface of a surface.

that $C^{\vee}$ has degree $d(d-1)$. Let $\kappa$ and $\delta$ be the number of cusps and nodes of $C^{\vee}$, respectively. Applying Lemma 5.15 to the plane curve $C^{\vee}$ yields

$$
d=\operatorname{deg}(C)=\operatorname{deg}\left(\left(C^{\vee}\right)^{\vee}\right)=d(d-1)(d(d-1)-1)-3 \kappa-2 \delta .
$$

The dual curves $C$ and $C^{\vee}$ have the same geometric genus; see [103, Prop. 1.5]. Hence, the genus-degree formula [93, p. 54, Eq. (7)] gives

$$
\begin{aligned}
\frac{1}{2}(d-1)(d-2) & =\operatorname{genus}(C) \\
& =\operatorname{genus}\left(C^{\vee}\right)=\frac{1}{2}(d(d-1)-1)(d(d-1)-2)-\kappa-\delta .
\end{aligned}
$$

Solving this system of two linear equations in $\kappa$ and $\delta$, we obtain $\kappa=3 d(d-2)$ and $\delta=\frac{1}{2} d(d-2)(d-3)(d+3)$.

The next result is the main theorem in this section and solves Problem 4 on Surfaces in [100]. The bidegrees of the bitangent and the inflectional congruence for a general smooth surface appear in [5, Prop. 3.3], and the bidegree of the inflectional congruence also appears in [77, Prop. 4.1].

Theorem 5.18. Let $S \subset \mathbb{P}^{3}$ be a general smooth irreducible surface of degree $d \geq 4$. The bidegree of $\operatorname{Bit}(S)$ is $\left(\frac{1}{2} d(d-1)(d-2)(d-3), \frac{1}{2} d(d-2)(d-3)(d+3)\right)$, and the bidegree of $\operatorname{Infl}(S)$ is $(d(d-1)(d-2), 3 d(d-2))$.

Proof. For a general plane $H \subset \mathbb{P}^{3}$, Bertini's Theorem [49, Thm. 17.16] implies that the intersection $S \cap H$ is a smooth plane curve of degree $d$. According to Proposition 5.17, the number of bitangents to $S$ contained in $H$ is $\frac{1}{2} d(d-2)(d-3)(d+3)$, which is the class of $\operatorname{Bit}(S)$. Similarly, the number of inflectional tangents to $S$ contained in $H$ is $3 d(d-2)$, which is the class of $\operatorname{Infl}(S)$.

It remains to calculate the number of bitangents and inflectional lines of the surface $S$ that pass through a general point $y \in \mathbb{P}^{3}$. Following the ideas in $[81$, p. 230], let $f$ be the defining equation for $S$ in $\mathbb{P}^{3}$ in the unknowns $x_{0}, \ldots, x_{3}$, and consider the polar curve $C:=P_{1}(S, y) \subset S$ with respect to the point $y$. 
Recall that the curve $C$ consists of all points $x \in S$ such that the line through $y$ and $x$ is tangent to $S$ at the point $x$; see Fig. 5.4. The condition that the point

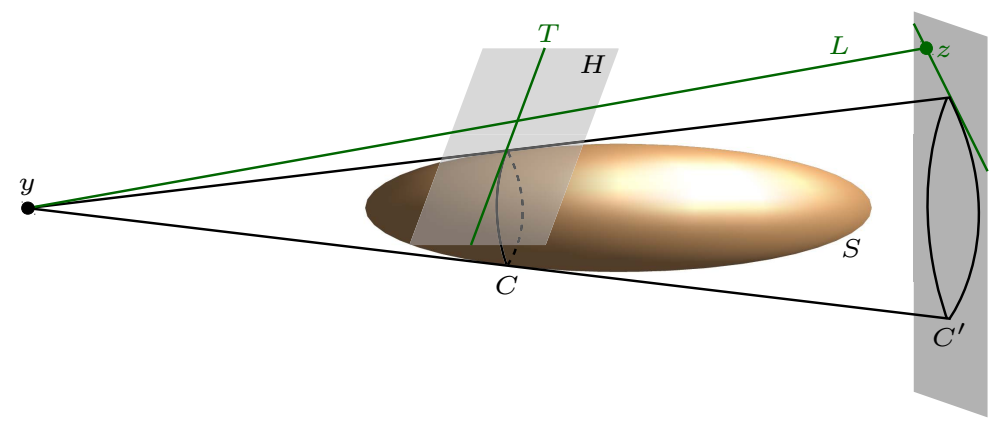

Figure 5.4: Polar curve.

$x$ lies on the curve $C$ is equivalent to saying that the point $y$ belongs to $\mathbb{T}_{S, x}$. As in the proof for Lemma 5.15, we have that $C$ is the zero locus $Z(f, g)$ where $g:=y_{0} \frac{\partial f}{\partial x_{0}}+y_{1} \frac{\partial f}{\partial x_{1}}+\cdots+y_{3} \frac{\partial f}{\partial x_{3}}$. Thus, the curve $C$ has degree $d(d-1)$.

Projecting away from the point $y$ gives the rational map $\pi_{y}: \mathbb{P}^{3} \rightarrow \mathbb{P}^{2}$. Restricted to the surface $S$, this map is generically finite, with fibers of cardinality $d$, and is ramified over the curve $C$. If $C^{\prime}$ is the image of $C$ under $\pi_{y}$, then a bitangent to the surface $S$ that passes through $y$ contains two points of $C$ and these points are mapped to a simple node in $C^{\prime}$; see Fig. 5.5. All of these nodes

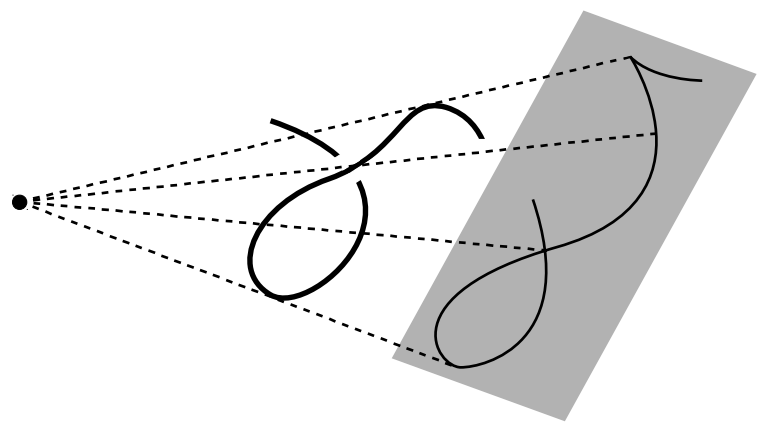

Figure 5.5: A secant projecting onto a node and a tangent projecting to a cusp.

in $C^{\prime}$ have two distinct tangent lines because no bitangent line passing through $y$ is contained in a bitangent plane that is tangent at the same two points as the line; the bitangent planes to $S$ form a one-dimensional family, so the union of bitangent lines they contain is a surface in $\mathbb{P}^{3}$ that does not contain the general point $y$.

We claim that the inflectional lines to $S$ passing through the point $y$ are exactly the tangent lines of $C$ passing through $y$. The line between a point $x \in S$ and the point $y$ is parametrized by the map $\ell: \mathbb{P}^{1} \rightarrow \mathbb{P}^{3}$ which sends the point $(s: t) \in \mathbb{P}^{1}$ to the point $\left(s x_{0}+t y_{0}: s x_{1}+t y_{1}: s x_{2}+t y_{2}: s x_{3}+t y_{3}\right) \in \mathbb{P}^{3}$. It follows that this line is an inflectional tangent to $S$ if and only if $f(\ell(s, t))$ is 
divisible by $t^{3}$. This is equivalent to the conditions that $\left.\frac{\partial}{\partial t}[f(\ell(s, t))]\right|_{(1,0)}=0$ and $\left.\frac{\partial^{2}}{\partial^{2} t}[f(\ell(s, t))]\right|_{(1,0)}=0$, which means that $x \in C$ and $y_{0} \frac{\partial g}{\partial x_{0}}+y_{1} \frac{\partial g}{\partial x_{1}}+\cdots+$ $y_{3} \frac{\partial g}{\partial x_{3}}=0$, or in other words $y \in \mathbb{T}_{C, x}$. Therefore, the inflectional lines to $S$ passing through $y$ are the tangents to $C$ passing through $y$, and are mapped to the cusps of $C^{\prime}$; again see Fig. 5.5.

Since the bitangent and inflectional lines to $S$ passing through $y$ correspond to nodes and cusps of $C^{\prime}$, it suffices to count the number $\kappa^{\prime}$ of cusps and the number $\delta^{\prime}$ of simple nodes in the plane curve $C^{\prime}$. We subdivide these calculations as follows.

$\kappa^{\prime}=d(d-1)(d-2)$ : From our parametrization of the line through the points $x \in S$ and $y$, we see that this line is an inflectional tangent to $S$ if and only if $x \in Z(f, g, h)$ where $h:=y_{0} \frac{\partial g}{\partial x_{0}}+y_{1} \frac{\partial g}{\partial x_{1}}+\cdots+y_{3} \frac{\partial g}{\partial x_{3}}$. Since $\operatorname{deg}(h)=d-2$ and $S$ is general, the zero locus $Z(f, g, h)$ consists of $d(d-1)(d-2)$ points.

$\operatorname{deg}\left(\left(C^{\prime}\right)^{\vee}\right)=\operatorname{deg}\left(S^{\vee}\right)$ : By duality, the degree $d^{\prime}$ of the curve $\left(C^{\prime}\right)^{\vee}$ is the number of tangent lines to $C^{\prime} \subset \mathbb{P}^{2}$ passing through a general point $z \in \mathbb{P}^{2}$. The preimage of $z$ under the projection $\pi_{y}$ is a line $L \subset \mathbb{P}^{3}$ containing $y$; see Fig. 5.4. Hence, $d^{\prime}$ is the number of tangent lines to $C$ intersecting $L$ in a point different from $y$. For every line $T$ that is tangent to $C$ at a point $x$ and intersects the line $L$, it follows that the pair $L$ and $T$ spans the tangent plane of $S$ at the point $x$. On the other hand, given any plane $H$ which is tangent to $S$ at the point $x$ and contains $L$, we deduce that $x$ must lie on the polar curve $C$ and $H$ is spanned by $L$ and the tangent line to $C$ at $x$, so this tangent line intersects $L$. Therefore, $d^{\prime}$ is the number of tangent planes to $S$ containing $L$, which is the degree of the dual surface $S^{\vee}$.

$\operatorname{deg}\left(S^{\vee}\right)=d(d-1)^{2}$ : By duality, the degree of $S^{\vee}$ is the number of tangent planes to the surface $S$ containing a general line, or the number of tangent planes to $S$ containing two general points $y, z \in \mathbb{P}^{3}$. Thus, this is the number of intersection points of the two polar curves of $S$ determined by $y$ and $z$, which is the cardinality of the zero locus $Z(f, g, \tilde{g})$ where $\tilde{g}:=z_{0} \frac{\partial f}{\partial x_{0}}+z_{1} \frac{\partial f}{\partial x_{1}}+\cdots+z_{3} \frac{\partial f}{\partial x_{3}}$. Since $\operatorname{deg}(\tilde{g})=d-1$, we conclude that $\operatorname{deg}\left(S^{\vee}\right)=d(d-1)^{2}$.

Finally, both the surface $S$ and the point $y$ are general, so Lemma 5.15 implies that $d(d-1)^{2}=\operatorname{deg}\left(\left(C^{\prime}\right)^{\vee}\right)=\operatorname{deg}\left(C^{\prime}\right)\left(\operatorname{deg}\left(C^{\prime}\right)-1\right)-3 d(d-1)(d-2)-2 \delta^{\prime}$. Since $\operatorname{deg}\left(C^{\prime}\right)=\operatorname{deg}(C)=d(d-1)$, we have $\delta^{\prime}=\frac{1}{2} d(d-1)(d-2)(d-3)$.

We end this section by proving that the secant locus of an irreducible smooth nondegenerate curve is isomorphic to the bitangent congruence of its dual surface via the natural isomorphism between $\operatorname{Gr}\left(1, \mathbb{P}^{3}\right)$ and $\operatorname{Gr}\left(1,\left(\mathbb{P}^{3}\right)^{*}\right)$. Analogously, the bitangent congruences of two surfaces which are projectively dual 
to each other are naturally isomorphic as well. Note that for every congruence $\Sigma \subset \operatorname{Gr}\left(1, \mathbb{P}^{3}\right)$ with bidegree $(\alpha, \beta)$, the bidegree of $\Sigma^{\perp} \subset \operatorname{Gr}\left(1,\left(\mathbb{P}^{3}\right)^{*}\right)$ is $(\beta, \alpha)$.

Proposition 5.19. If $C \subset \mathbb{P}^{3}$ is a smooth nondegenerate irreducible curve, then we have $\operatorname{Sec}(C)^{\perp}=\operatorname{Bit}\left(C^{\vee}\right)$, the inflectional lines of $C^{\vee}$ are dual to the tangent lines of $C$, and $\operatorname{Infl}\left(C^{\vee}\right) \subset \operatorname{Bit}\left(C^{\vee}\right)$.

Proof. We first show that $\operatorname{Sec}(C)^{\perp}=\operatorname{Bit}\left(C^{\vee}\right)$. As in the proof of Theorem 3.13 . the biduality of projective varieties over algebraically closed fields of characteristic zero is the main ingredient. Consider a line $L$ that intersects $C$ at two distinct points $x$ and $y$, but is equal to neither $\mathbb{T}_{C, x}$ nor $\mathbb{T}_{C, y}$. Together the line $L$ and $\mathbb{T}_{C, x}$ span a plane corresponding to a point $a \in C^{\vee}$. Similarly, the span of the lines $L$ and $\mathbb{T}_{C, y}$ corresponds to a point $b \in C^{\vee}$. Without loss of generality, we may assume that both $a$ and $b$ are smooth points in $C^{\vee}$. By biduality, the points $a, b \in C^{\vee}$ must be distinct with tangent planes corresponding to $x$ and $y$. Thus, the line $L^{\vee}$ is tangent to $C^{\vee}$ at the points $a, b$, and $\operatorname{Sec}(C)^{\perp} \subset \operatorname{Bit}\left(C^{\vee}\right)$. To establish the other inclusion, let $L^{\prime}$ be a line that is tangent to $C^{\vee}$ at two distinct smooth points $a, b \in C^{\vee}$. The tangent planes at the points $a, b$ correspond to two points $x, y \in C$. If $x \neq y$, then $\left(L^{\prime}\right)^{\vee}$ is the secant to $C$ through these two points. If $x=y$, then biduality establishes that $\left(L^{\prime}\right)^{\vee}$ is the tangent line of $C$ at $x$. In either case, we see that $\operatorname{Bit}\left(C^{\vee}\right) \subset \operatorname{Sec}(C)^{\perp}$, so $\operatorname{Sec}(C)^{\perp}=\operatorname{Bit}\left(C^{\vee}\right)$.

For the second part, let $L$ be an inflectional line at a smooth point $a \in C^{\vee}$. A point $y \in L^{\vee} \backslash C$ corresponds to a plane $H$ such that $L=\mathbb{T}_{C^{\vee}, a} \cap H$, so the line $L$ is also an inflectional line to the plane curve $C^{\vee} \cap H \subset H$. Regarding $L$ as a subvariety of the projective plane $H$, its dual variety is a cusp on the plane curve $\left(C^{\vee} \cap H\right)^{\vee} \subset H^{*}$; see Fig. 5.2. If $\pi_{y}: \mathbb{P}^{3} \rightarrow \mathbb{P}^{2} \cong H^{*}$ denotes the projection away from the point $y$, then we claim that $\left(C^{\vee} \cap H\right)^{\vee}$ equals $\pi_{y}(C)$; for a more general version see [53, Prop. 6.1]. Indeed, a smooth point $z \in \pi_{y}(C)$ is the projection of a point of $C$ whose tangent line does not contain $y$. Together this tangent line and the point $y$ span a plane such that its dual point $w$ is contained in the curve $C^{\vee} \cap H$. Thus, the tangent line $\mathbb{T}_{\pi_{y}(C), z}$ equals $\pi_{y}\left(w^{\vee}\right)$; the latter is the line in $H^{*}$ dual to the point $w \in H$. In other words, we have $\left(\pi_{y}(C)\right)^{\vee} \subset C^{\vee} \cap H$. Since both curves are irreducible, this inclusion must be an equality. Hence, when considering $L$ in the projective plane $H$, its dual point is a cusp of $\pi_{y}(C)$. It follows that $L^{\vee}$ is the tangent line $\mathbb{T}_{C, x}$, where $x \in C$ is the point corresponding to the tangent plane $\mathbb{T}_{C^{\vee}, a}$; see Fig. 5.5. Reversing these arguments shows that the dual of a tangent line to $C$ is an inflectional line to $C^{\vee}$. Since every tangent line to $C$ is contained in $\operatorname{Sec}(C)$, we conclude that $\operatorname{Infl}\left(C^{\vee}\right) \subset \operatorname{Bit}\left(C^{\vee}\right)$.

Proposition 5.20. Let $S \subset \mathbb{P}^{3}$ be an irreducible surface. If $S^{\vee}$ is a surface as well, then $\operatorname{Bit}(S)^{\perp}=\operatorname{Bit}\left(S^{\vee}\right)$.

Proof. A general $L \in \operatorname{Bit}(S)$ is tangent to $S$ at two distinct smooth points $x$ and $y$ such that $a:=\left(\mathbb{T}_{S, x}\right)^{\vee}$ and $b:=\left(\mathbb{T}_{S, y}\right)^{\vee}$ are smooth point of $S^{\vee}$. Biduality implies that $a$ and $b$ must be distinct and that $L^{\vee}$ is tangent to $S^{\vee}$ at both $a$ and 
$b$. Now we have shown that $\operatorname{Bit}(S)^{\perp} \subset \operatorname{Bit}\left(S^{\vee}\right)$. Repeating the same argument with the roles of $S$ and $S^{\vee}$ exchanged yields Proposition 5.20.

Proof of Theorem 5.2 This result is a restatement of Propositions 5.19 and 5.20

Remark 5.21. Proposition 5.19 shows, for a smooth nondegenerate irreducible space curve $C$, that $\operatorname{Infl}\left(C^{\vee}\right)$ is a curve, as $\operatorname{Infl}\left(C^{\vee}\right)^{\perp}$ is the set of tangent lines to $C$; so the inflectional locus of a surface in $\mathbb{P}^{3}$ is not always a congruence.

Remark 5.22. For a curve $C \subset \mathbb{P}^{3}$ with dual surface $C^{\vee} \subset\left(\mathbb{P}^{3}\right)^{*}$, Theorem 3.13 establishes that $\mathrm{CH}_{0}(C)^{\perp}=\mathrm{CH}_{1}\left(C^{\vee}\right)$. Combined with Proposition 5.19, we see that the singular locus of the Hurwitz hypersurface $\mathrm{CH}_{1}\left(C^{\vee}\right)$, for smooth and nondegenerate $C$, has just one component, namely the bitangent congruence. $\diamond$ Remark 5.23. Proposition 5.19 does not hold for degenerate curves. If $C \subset \mathbb{P}^{3}$ is an irreducible curve which spans the plane $H \subset \mathbb{P}^{3}$, then its secant congruence is the set of all lines contained in $H$, but the bitangent congruence of the dual surface $C^{\vee}$ does not consist of all lines passing through the point $H^{\vee}$. Note that $C^{\vee}$ is a cone with vertex $H^{\vee}$, whose image under the projection $\pi_{v}$ with center $v:=H^{\vee}$ is the dual curve to the plane curve $C \subset H \cong \mathbb{P}^{2}$. There are two types of bitangent lines of $C^{\vee}$ : lines contained in the cone $C^{\vee}$ (which are dual to the tangent lines of $C$ ) and lines whose image under $\pi_{v}$ are bitangent lines of the plane curve $\pi_{v}\left(C^{\vee}\right)$. Conversely, given a bitangent line $L$ of the plane curve $\pi_{v}\left(C^{\vee}\right)$, almost all lines in the plane $\pi_{v}^{-1}(L)$ are bitangent lines of $C^{\vee}$, so all lines in the plane $\pi_{v}^{-1}(L)$ are contained in $\operatorname{Bit}\left(C^{\vee}\right)$. Since bitangent lines of the plane curve $\pi_{v}\left(C^{\vee}\right)$ correspond to nodes of $C$, we have derived the following:

$$
\operatorname{Bit}\left(C^{\vee}\right)^{\perp}=\mathcal{T}^{\ell}(C) \cup \bigcup_{x \text { node of } C}\left\{L \in \operatorname{Gr}\left(1, \mathbb{P}^{3}\right) \mid x \in L\right\}
$$

where $\mathcal{T}^{\ell}(C)$ denotes the Zariski closure of the set of tangent lines to smooth points of $C$. Analogously, since inflectional lines of a plane curve correspond to cusps of its dual curve, we have

$$
\operatorname{Infl}\left(C^{\vee}\right)^{\perp}=\mathcal{T}^{\ell}(C) \cup \bigcup_{x \text { cusp of } C}\left\{L \in \operatorname{Gr}\left(1, \mathbb{P}^{3}\right) \mid x \in L\right\} .
$$

Similarly, for nondegenerate singular curves, the bitangent and inflectional loci of their dual surfaces may have several components each. For example, if such a curve $C$ has a node $x$, then all lines in the plane $x^{\vee}$ correspond to points of $\operatorname{Bit}\left(C^{\vee}\right)$, but according to Remark 5.9, not all lines passing through the point $x$ correspond to points of $\operatorname{Sec}(C)$ (cf. also Theorem 5.8).

\subsection{Intersection Theory}

In this section, we recast the degree of a subvariety in $\operatorname{Gr}\left(1, \mathbb{P}^{3}\right)$ in terms of certain products in the Chow ring. For information about subvarieties of more 
general Grassmannians, we recommend [4].

Consider a smooth irreducible variety $X$. For each $c \in \mathbb{Z}_{\geq 0}$, the group $Z^{c}(X)$ of codimension- $c$ cycles is the free abelian group generated by the closed irreducible subvarieties of $X$ having codimension $c$. Given a variety $W$ of codimension $c-1$ and a non-zero rational function $f$ on $W$, we have the cycle $\operatorname{div}(f):=\sum_{Z} \operatorname{ord}_{Z}(f) Z$ where the sum runs over all subvarieties $Z$ of $W$ with codimension one in $W$ and $\operatorname{ord}_{Z}(f) \in \mathbb{Z}$ is the order of vanishing of $f$ along $Z$. The group of cycles rationally equivalent to zero is the subgroup generated by the cycles $\operatorname{div}(f)$ for all codimension- $(c-1)$ subvarieties $W$ of $X$ and all nonzero rational functions $f$ on $W$. The Chow group $A^{c}(X)$ is the quotient of $Z^{c}(X)$ by the subgroup of cycles rationally equivalent to zero. We typically write $[Z]$ for the class of a subvariety $Z$ in the appropriate Chow group. Since $X$ is the unique subvariety of codimension 0 , we see that $A^{0}(X) \cong \mathbb{Z}$. We also have $A^{1}(X) \cong \operatorname{Pic}(X)$.

Theorem/Definition 5.24 ([34, Thm.-Def. 1.5]). If $X$ is a smooth projective variety, then there is a unique product structure on $A^{*}(X)=\bigoplus_{c=0}^{\operatorname{dim} X} A^{c}(X)$ which satisfies the condition:

If two subvarieties $Y_{1}, Y_{2} \subset X$ are generically transverse, then $\left[Y_{1}\right]\left[Y_{2}\right]=\left[Y_{1} \cap Y_{2}\right]$.

This makes $A^{*}(X)$ into an associative commutative $\mathbb{Z}$-graded ring, called the Chow ring of $X$.

Example 5.25 ([34, Thm. 2.1]). The Chow ring of $\mathbb{P}^{n}$ is isomorphic to $\mathbb{Z}[h] /\left\langle h^{n+1}\right\rangle$, where $h \in A^{1}\left(\mathbb{P}^{n}\right)$ is the (rational equivalence) class of a hyperplane in $\mathbb{P}^{n}$. More generally, any subvariety of codimension $c$ and degree $d$ is rationally equivalent to the $d$-times multiple of the intersection of $c$ hyperplanes.

To a given a vector bundle $\mathcal{E}$ of rank $r$ on $X$, we associate its Chern classes $c_{i}(\mathcal{E}) \in A^{i}(X)$ for $0 \leq i \leq r$; see [102]. When $\mathcal{E}$ is globally generated, these classes are represented by degeneracy loci; the class $c_{r+1-j}(\mathcal{E})$ is associated to the locus of points $x \in X$ where $j$ general global sections of $\mathcal{E}$ fail to be linearly independent. In particular, the top Chern class $c_{r}(\mathcal{E})$ is represented by the vanishing locus of a single general global section. Given a short exact sequence $0 \rightarrow \mathcal{E}^{\prime} \rightarrow \mathcal{E} \rightarrow \mathcal{E}^{\prime \prime} \rightarrow 0$ of vector bundles, the Whitney Sum Formula asserts that $c_{k}(\mathcal{E})=\sum_{i+j=k} c_{i}\left(\mathcal{E}^{\prime}\right) c_{j}\left(\mathcal{E}^{\prime \prime}\right)$; see [40, Thm. 3.2]. Moreover, if $\mathcal{E}^{*}:=$ $\mathcal{H} \operatorname{lom}\left(\mathcal{E}, \mathcal{O}_{X}\right)$ denotes the dual vector bundle, then we have $c_{i}\left(\mathcal{E}^{*}\right)=(-1)^{i} c_{i}(\mathcal{E})$ for $0 \leq i \leq r$; see [40, Rem. 3.2.3].

Example 5.26. Given nonnegative integers $a_{1}, a_{2}, \ldots, a_{n}$, consider the vector bundle $\mathcal{E}:=\mathcal{O}_{\mathbb{P}^{n}}\left(a_{1}\right) \oplus \mathcal{O}_{\mathbb{P}^{n}}\left(a_{2}\right) \oplus \cdots \oplus \mathcal{O}_{\mathbb{P}^{n}}\left(a_{n}\right)$. Since each $\mathcal{O}_{\mathbb{P}^{n}}\left(a_{i}\right)$ is globally generated, the Chern class $c_{1}\left(\mathcal{O}_{\mathbb{P}^{n}}\left(a_{i}\right)\right)$ is the vanishing locus of a general homogeneous polynomial of degree $a_{i}$, so $c_{1}\left(\mathcal{O}_{\mathbb{P}^{n}}\left(a_{i}\right)\right)=a_{i} h$ in $A^{*}\left(\mathbb{P}^{n}\right)$. Hence, the Whitney Sum Formula implies that $c_{n}(\mathcal{E})=\prod_{i=1}^{n} c_{1}\left(\mathcal{O}\left(a_{i}\right)\right)=\prod_{i=1}^{n}\left(a_{i} h\right)$.

On the one hand, $c_{n}(\mathcal{E})$ is the locus where a general section vanishes. In other words, it is the common zeros of general polynomials of degree $a_{1}, a_{2}, \ldots, a_{n}$. 
On the other hand, it is represented by $\prod_{i=1}^{n} a_{i}$ times the point $h^{n}$. Thus we have recovered Bézout's Theorem in the case of general polynomials.

Example 5.27. If $\mathcal{T}_{\mathbb{P}^{n}}$ is the tangent bundle on $\mathbb{P}^{n}$, then we have the short exact sequence $0 \rightarrow \mathcal{O}_{\mathbb{P}^{n}} \rightarrow \mathcal{O}_{\mathbb{P}^{n}}(1)^{\oplus(n+1)} \rightarrow \mathcal{T}_{\mathbb{P}^{n}} \rightarrow 0$; see [50, Example 8.20.1]. The Whitney Sum Formula implies that $c_{1}\left(\mathcal{T}_{\mathbb{P}^{n}}\right)=(n+1) c_{1}\left(\mathcal{O}_{\mathbb{P}^{n}}(1)\right)-c_{1}\left(\mathcal{O}_{\mathbb{P}^{n}}\right)=$ $(n+1) h$ and $c_{2}\left(\mathcal{T}_{\mathbb{P}^{n}}\right)=c_{2}\left(\mathcal{O}_{\mathbb{P}^{n}}(1)^{\oplus(n+1)}\right)=\left(\begin{array}{c}n+1 \\ 2\end{array}\right) h^{2}$.

Example 5.28. Let $Y \subset \mathbb{P}^{n}$ be a smooth hypersurface of degree $d$. If $\mathcal{T}_{Y}$ denotes the tangent bundle of $Y$, then we have the following short exact sequence: $\left.\left.0 \rightarrow \mathcal{T}_{Y} \rightarrow \mathcal{T}_{\mathbb{P}^{n}}\right|_{Y} \rightarrow \mathcal{O}_{\mathbb{P}^{n}}(d)\right|_{Y} \rightarrow 0$; see [50, Prop. 8.20]. Setting $H:=\left.h\right|_{Y}$ in $A^{*}(Y)$, the Whitney Sum Formula implies

$$
\begin{aligned}
& c_{1}\left(\mathcal{T}_{Y}\right)=c_{1}\left(\left.\mathcal{T}_{\mathbb{P}^{n}}\right|_{Y}\right)-c_{1}\left(\left.\mathcal{O}_{\mathbb{P}^{n}}(d)\right|_{Y}\right)=(n+1) H-d H=(n+1-d) H \quad \text { and } \\
& c_{2}\left(\mathcal{T}_{Y}\right)=c_{2}\left(\left.\mathcal{T}_{\mathbb{P}^{n}}\right|_{Y}\right)-c_{1}\left(\mathcal{T}_{Y}\right) c_{1}\left(\left.\mathcal{O}_{\mathbb{P}^{n}}(d)\right|_{Y}\right)=\left(\left(\begin{array}{c}
n+1 \\
2
\end{array}\right)-(n+1-d) d\right) H^{2} .
\end{aligned}
$$

We next focus on the Chow ring of $\operatorname{Gr}\left(1, \mathbb{P}^{3}\right)$; see [4, 102]. Fix a complete flag $v_{0} \in L_{0} \subset H_{0} \subset \mathbb{P}^{3}$ where the point $v_{0}$ lies in the line $L_{0}$, and the line $L_{0}$ is contained in the plane $H_{0}$. The Schubert varieties in $\operatorname{Gr}\left(1, \mathbb{P}^{3}\right)$ are:

$$
\begin{aligned}
\Sigma_{0}:=\operatorname{Gr}\left(1, \mathbb{P}^{3}\right), & \Sigma_{1}:=\left\{L \mid L \cap L_{0} \neq \varnothing\right\} \subset \operatorname{Gr}\left(1, \mathbb{P}^{3}\right), \\
\Sigma_{1,1}:=\left\{L \mid L \subset H_{0}\right\} \subset \operatorname{Gr}\left(1, \mathbb{P}^{3}\right), & \Sigma_{2}:=\left\{L \mid v_{0} \in L\right\} \subset \operatorname{Gr}\left(1, \mathbb{P}^{3}\right), \\
\Sigma_{2,1}:=\left\{L \mid v_{0} \in L \subset H_{0}\right\} \subset \operatorname{Gr}\left(1, \mathbb{P}^{3}\right), & \Sigma_{2,2}:=\left\{L_{0}\right\} \subset \operatorname{Gr}\left(1, \mathbb{P}^{3}\right) .
\end{aligned}
$$

The corresponding classes $\sigma_{I}:=\left[\Sigma_{I}\right]$, called the Schubert cycles, form a basis for the Chow ring $A^{*}\left(\operatorname{Gr}\left(1, \mathbb{P}^{3}\right)\right)$; see [34, Thm. 5.26]. Since the sum of the subscripts gives the codimension, we have

$$
\begin{aligned}
& A^{0}\left(\operatorname{Gr}\left(1, \mathbb{P}^{3}\right)\right) \cong \mathbb{Z} \sigma_{0}, \quad A^{1}\left(\operatorname{Gr}\left(1, \mathbb{P}^{3}\right)\right) \cong \mathbb{Z} \sigma_{1}, \quad A^{2}\left(\operatorname{Gr}\left(1, \mathbb{P}^{3}\right)\right) \cong \mathbb{Z} \sigma_{1,1} \oplus \mathbb{Z} \sigma_{2}, \\
& A^{3}\left(\operatorname{Gr}\left(1, \mathbb{P}^{3}\right)\right) \cong \mathbb{Z} \sigma_{2,1}, \quad A^{4}\left(\operatorname{Gr}\left(1, \mathbb{P}^{3}\right)\right) \cong \mathbb{Z} \sigma_{2,2} .
\end{aligned}
$$

To understand the product structure, we use the transitive action of $G L(4, \mathbb{C})$ on $\operatorname{Gr}\left(1, \mathbb{P}^{3}\right)$. Specifically, Kleiman's Transversality Theorem [60] shows that, for two subvarieties $V$ and $W$ in $\operatorname{Gr}\left(1, \mathbb{P}^{3}\right)$, a general translate $U$ of $V$ under the $\mathrm{GL}(4, \mathbb{C})$-action is rationally equivalent to $V$ and the intersection of $U$ and $W$ is transversal at a general point of any component of $U \cap W$. Hence, we have $[V][W]=[U \cap W]$. To determine the product $\sigma_{1,1} \sigma_{2}$, we intersect general varieties representing these classes: $\sigma_{1,1}$ consists of all lines $L$ contained in a fixed plane $H_{0}$, and $\sigma_{2}$ is all lines $L$ containing a fixed point $v_{0}$. Since a general point does not lie in a general plane, we see that $\sigma_{1,1} \sigma_{2}=0$. Similar arguments yield all products:

$$
\begin{aligned}
& \sigma_{1,1}^{2}=\sigma_{2,2}, \quad \sigma_{2}^{2}=\sigma_{2,2}, \quad \sigma_{1,1} \sigma_{2}=0, \quad \sigma_{1} \sigma_{2,1}=\sigma_{2,2} \text { ， } \\
& \sigma_{1} \sigma_{1,1}=\sigma_{2,1}, \quad \sigma_{1} \sigma_{2}=\sigma_{2,1}, \quad \sigma_{1}^{2}=\sigma_{2}+\sigma_{1,1} .
\end{aligned}
$$


The degree of a subvariety in $\operatorname{Gr}\left(1, \mathbb{P}^{3}\right)$ can be interpreted as certain coefficients of its class in the Chow ring. Geometrically, the order $\alpha$ of a surface $X \subset \operatorname{Gr}\left(1, \mathbb{P}^{3}\right)$ is the number of lines in $X$ passing through the general point $v_{0}$. Since we may intersect $X$ with a general variety representing $\sigma_{2}$, it follows that $\alpha$ equals the coefficient of $\sigma_{2}$ in $[X]$. Similarly, the class $\beta$ of $X$ is the coefficient of $\sigma_{1,1}$ in $[X]$, the degree of a threefold $\Sigma \subset \operatorname{Gr}\left(1, \mathbb{P}^{3}\right)$ is the coefficient of $\sigma_{1}$ in $[\Sigma]$, and the degree of a curve $C \subset \operatorname{Gr}\left(1, \mathbb{P}^{3}\right)$ is the coefficient of $\sigma_{2,1}$ in $[C]$.

The degree of a subvariety in $\operatorname{Gr}\left(1, \mathbb{P}^{3}\right)$ also has a useful reinterpretation via Chern classes of tautological vector bundles. Let $\mathcal{S}$ denote the tautological subbundle, the vector bundle whose fiber over the point $W \in \operatorname{Gr}\left(1, \mathbb{P}^{3}\right)$ is the twodimensional vector space $W \subseteq \mathbb{A}^{4}$. Similarly, let $\mathcal{Q}$ be the tautological quotient bundle whose fiber over $W$ is $\mathbb{A}^{4} / W$. Both $\mathcal{S}^{*}$ and $\mathcal{Q}$ are globally generated; $H^{0}\left(\operatorname{Gr}\left(1, \mathbb{P}^{3}\right), \mathcal{S}^{*}\right) \cong\left(\mathbb{A}^{4}\right)^{*}$ and $H^{0}\left(\operatorname{Gr}\left(1, \mathbb{P}^{3}\right), \mathcal{Q}\right) \cong \mathbb{A}^{4}$; see [4, Prop. 0.5]. A global section of $\mathcal{S}^{*}$ corresponds to a non-zero map $\varphi: \mathbb{A}^{4} \rightarrow \mathbb{A}^{1}$, where its value at the point $W$ is $\left.\varphi\right|_{W}: W \rightarrow \mathbb{A}^{1}$. The Chern class $c_{2}\left(\mathcal{S}^{*}\right)$ is represented by the vanishing locus of $\varphi$, so we have $c_{2}\left(\mathcal{S}^{*}\right)=\sigma_{1,1}=c_{2}(\mathcal{S})$. For two general sections $\varphi, \psi: \mathbb{A}^{4} \rightarrow \mathbb{A}^{1}$ of $\mathcal{S}^{*}$, the Chern class $c_{1}\left(\mathcal{S}^{*}\right)$ is represented by the locus of points $W$ where $\left.\varphi\right|_{W}$ and $\left.\psi\right|_{W}$ fail to be linearly independent, i.e., $W \cap \operatorname{ker}(\varphi) \cap \operatorname{ker}(\psi) \neq\{0\}$. Generality ensures that $\operatorname{ker}(\varphi) \cap \operatorname{ker}(\psi)$ is a twodimensional subspace of $\mathbb{A}^{4}$, so $c_{1}\left(\mathcal{S}^{*}\right)=-c_{1}(\mathcal{S})=\sigma_{1}$. Similarly, a global section of $\mathcal{Q}$ corresponds to a point $v \in \mathbb{A}^{4}$; its value at $W$ is simply the image of the point in $\mathbb{A}^{4} / W$. Thus, $c_{2}(\mathcal{Q})$ is represented by the locus of those $W$ containing $v$, which is $\sigma_{2}$. Two global sections of $\mathcal{Q}$ are linearly dependent at $W$ when the two-dimensional subspace of $\mathbb{A}^{4}$ spanned by the points intersects $W$ nontrivially, so $c_{1}(\mathcal{Q})=\sigma_{1}$. Finally, for a surface $X \subset \operatorname{Gr}\left(1, \mathbb{P}^{3}\right)$ with $[X]=\alpha \sigma_{2}+\beta \sigma_{1,1}$, we obtain

$$
\begin{aligned}
& c_{2}(\mathcal{Q})[X]=\sigma_{2}\left(\alpha \sigma_{2}+\beta \sigma_{1,1}\right)=\alpha \sigma_{2,2}, \\
& \mathcal{c}_{2}(\mathcal{S})[X]=\sigma_{1,1}\left(\alpha \sigma_{2}+\beta \sigma_{1,1}\right)=\beta \sigma_{2,2},
\end{aligned}
$$

so computing the bidegree is equivalent to calculating the products $c_{2}(\mathcal{Q})[X]$ and $c_{2}(\mathcal{S})[X]$ in the Chow ring.

We close this section with three examples demonstrating this approach. Further elaboration and examples of intersection theory on the Grassmannian $\operatorname{Gr}\left(1, \mathbb{P}^{3}\right)$ are found in Section 6.4

Example 5.29. Given a smooth surface $S$ in $\mathbb{P}^{3}$, we recompute the degree of $\mathrm{CH}_{1}(S)$; compare with Proposition 5.16. Theorem 3.8 implies that this degree equals the degree $\delta_{1}(S)$ of the first polar locus $P_{1}(S, y)=\left\{x \in S \mid y \in \mathbb{T}_{S, x}\right\}$, where $y$ is a general point of $\mathbb{P}^{3}$. Letting $\mathcal{T}_{S}$ be the tangent bundle of $S$, Example 14.4.15 in [40] shows that $\delta_{1}(S)=\operatorname{deg}\left(3 h-c_{1}\left(\mathcal{T}_{S}\right)\right)$. Hence, Example 5.28 gives $\delta_{1}(S)=\operatorname{deg}(3 h-h(3+1-d))=(d-1) \operatorname{deg}(h)$. Since $S$ is a degree $d$ surface, the degree of the hyperplane $h$ equals $d$, so $\delta_{1}(S)=d(d-1)$.

Example 5.30 (Problem 3 on Grassmannians in [100]). Let $S_{1}, S_{2} \subset \mathbb{P}^{3}$ be general surfaces of degree $d_{1}$ and $d_{2}$, respectively, with $d_{1}, d_{2} \geq 4$. To find 
the number of lines bitangent to both surfaces, it suffices to compute the cardinality of $\operatorname{Bit}\left(S_{1}\right) \cap \operatorname{Bit}\left(S_{2}\right)$. Theorem 5.18 establishes that, for $1 \leq i \leq 2$, we have $\left[\operatorname{Bit}\left(S_{i}\right)\right]=\alpha_{i} \sigma_{2}+\beta_{i} \sigma_{1,1}$, where $\alpha_{i}:=\frac{1}{2} d_{i}\left(d_{i}-1\right)\left(d_{i}-2\right)\left(d_{i}-3\right)$ and $\beta_{i}:=\frac{1}{2} d_{i}\left(d_{i}-2\right)\left(d_{i}-3\right)\left(d_{i}+3\right)$. So $\left[\operatorname{Bit}\left(S_{1}\right) \cap \operatorname{Bit}\left(S_{2}\right)\right]=\left[\operatorname{Bit}\left(S_{1}\right)\right]\left[\operatorname{Bit}\left(S_{2}\right)\right]=$ $\left(\alpha_{1} \alpha_{2}+\beta_{1} \beta_{2}\right) \sigma_{2,2}$ and the number of lines bitangent to $S_{1}$ and $S_{2}$ is

$$
\begin{array}{r}
\quad \frac{1}{4} d_{1}\left(d_{1}-1\right)\left(d_{1}-2\right)\left(d_{1}-3\right) d_{2}\left(d_{2}-1\right)\left(d_{2}-2\right)\left(d_{2}-3\right) \\
+\frac{1}{4} d_{1}\left(d_{1}-2\right)\left(d_{1}-3\right)\left(d_{1}+3\right) d_{2}\left(d_{2}-2\right)\left(d_{2}-3\right)\left(d_{2}+3\right) .
\end{array}
$$

Example 5.31. Let $S \subset \mathbb{P}^{3}$ be a general surface of degree $d_{1}$ with $d_{1} \geq 4$, and let $C \subset \mathbb{P}^{3}$ be a general curve of degree $d_{2}$ and geometric genus $g$ with $d_{2} \geq 2$. To find the number of lines bitangent to $S$ and secant to $C$, it suffices to compute the cardinality of $\operatorname{Bit}(S) \cap \operatorname{Sec}(C)$. Theorem 5.18 and Theorem 5.10 imply that

$$
\begin{aligned}
{[\operatorname{Bit}(S)] } & =\frac{1}{2} d_{1}\left(d_{1}-1\right)\left(d_{1}-2\right)\left(d_{1}-3\right) \sigma_{2}+\frac{1}{2} d_{1}\left(d_{1}-2\right)\left(d_{1}-3\right)\left(d_{1}+3\right) \sigma_{1,1}, \\
{[\operatorname{Sec}(C)] } & =\left(\frac{1}{2}\left(d_{2}-1\right)\left(d_{2}-2\right)-g\right) \sigma_{2}+\frac{1}{2} d_{2}\left(d_{2}-1\right) \sigma_{1,1} .
\end{aligned}
$$

It follows that $[\operatorname{Bit}(S) \cap \operatorname{Sec}(C)]=[\operatorname{Bit}(S)][\operatorname{Sec}(C)]=\gamma \sigma_{2,2}$ where

$$
\begin{aligned}
\gamma:=\frac{1}{4} d_{1}\left(d_{1}-1\right)\left(d_{1}-2\right)\left(d_{1}-3\right) & \left(\left(d_{2}-1\right)\left(d_{2}-2\right)-2 g\right) \\
& +\frac{1}{4} d_{1}\left(d_{1}-2\right)\left(d_{1}-3\right)\left(d_{1}+3\right) d_{2}\left(d_{2}-1\right),
\end{aligned}
$$

so the number of lines bitangent to $S$ and secant to $C$ is $\gamma$.

\subsection{Singular Loci of Congruences}

This section investigates the singular points of the secant, bitangent, and inflectional congruences. We begin with the singularities of the secant locus of a smooth irreducible curve that is not contained in a plane. Note that the secant congruence of a curve that is not a line but contained in a plane is simply the set of all lines lying in the same plane; hence this congruence is smooth.

Proposition 5.32. Let $C$ be a nondegenerate smooth irreducible curve in $\mathbb{P}^{3}$. If $L$ is a line that intersects the curve $C$ in three or more distinct points, then the line $L$ corresponds to a singular point in $\operatorname{Sec}(C)$.

Proof. The symmetric square $C^{(2)}$ is the quotient of $C \times C$ by the action of the symmetric group $\mathfrak{S}_{2}$, so points in this projective variety are unordered pairs of points on $C$; see [49, pp. 126-127]. The map $\omega: C^{(2)} \rightarrow \operatorname{Sec}(C)$, defined by sending $\{x, y\}$ to the line spanned by the points $x$ and $y$ if $x \neq y$ or to the tangent line $\mathbb{T}_{C, x}$ if $x=y$, is a birational morphism. Since $|L \cap C| \geq 3$, the fiber $\omega^{-1}(L)$ is a finite set containing more than one element. Hence, $\omega^{-1}(L)$ is not connected and the Zariski Connectedness Theorem proves that $\operatorname{Sec}(C)$ is singular at $L$.

We use the following result without proof. 
Proposition 5.33 ([5, Lem. 2.3]). Let $C$ be a nondegenerate smooth irreducible curve in $\mathbb{P}^{3}$ and $L$ be a line that intersects $C$ in exactly two points $x$ and $y$. The line $L$ corresponds to a smooth point of $\operatorname{Sec}(C)$ if and only if $L$ is different from $\mathbb{T}_{C, x}$ and $\mathbb{T}_{C, y}$.

Finally we have to consider lines in $\operatorname{Sec}(C)$ that meet the curve in a single point. For this, we need the following technical lemma.

Lemma 5.34. If $f \in \mathbb{C} \llbracket z, w \rrbracket$ satisfies $f(z, w)=-f(w, z)$, then the linear form $z-w$ divides the power series $f$.

Proof. We write the formal power series $f$ as a sum of homogeneous polynomials $f=\sum_{i \in \mathbb{Z}_{>0}} f_{i}$. Since we have $f(z, w)+f(w, z)=0$, it follows that, in each degree $i$, we have $f_{i}(z, w)+f_{i}(w, z)=0$. In particular, we see that $f_{i}(w, w)=0$. If we consider $f_{i}(w, z)$ as a polynomial in the variable $z$ with coefficients in $\mathbb{C}[w]$, it follows that $w$ is a root of $f_{i}$. Thus, we conclude that $z-w$ divides $f_{i}$ for all $i \in \mathbb{Z}_{\geq 0}$.

Theorem 5.35. Let $C$ be a nondegenerate smooth irreducible curve in $\mathbb{P}^{3}$. If a point in $\operatorname{Sec}(C)$ corresponds to a line $L$ that intersects $C$ in a single point $v$, then the intersection multiplicity of $L$ and $C$ at $v$ is at least two. Moreover, the line $L$ corresponds to a smooth point of $\operatorname{Sec}(C)$ if and only if the intersection multiplicity is exactly two.

Proof. First we note that Jenia Tevelev helped with the following proof.

Suppose the line $L$ intersects the curve $C$ at the point $v$ with multiplicity two. Without loss of generality, we may work in the affine open subset with $x_{3} \neq 0$, and we assume that $v=(0: 0: 0: 1)$ and $L$ is the zero locus $Z\left(x_{1}, x_{2}\right)$. Since $C$ is smooth, there is a local analytic isomorphism $\varphi$ from a neighborhood of the origin in $\mathbb{A}^{1}$ to a neighborhood of the point $v$ in $C$. The map $\varphi$ will have the form $\varphi(z)=\left(\varphi_{0}(z), \varphi_{1}(z), \varphi_{2}(z)\right)$ for some $\varphi_{0}, \varphi_{1}, \varphi_{2} \in \mathbb{C} \llbracket z \rrbracket$. We have $\varphi_{0}^{\prime}(0) \neq 0$ and $\varphi_{1}^{\prime}(0)=\varphi_{2}^{\prime}(0)=0$ because $L$ is the tangent to the curve $C$ at $v$. After making an analytic change of coordinates, we may assume that $\varphi(z)=\left(z, \varphi_{1}(z), \varphi_{2}(z)\right)$. As $L$ is a simple tangent, at least one of $\varphi_{1}$ and $\varphi_{2}$ must vanish at 0 with order exactly two. Hence, we may assume that $\varphi_{1}(z)=z^{2}+z^{3} f(z)$ and $\varphi_{2}(z)=$ $z^{2} g(z)$ for some $f, g \in \mathbb{C} \llbracket z \rrbracket$. The line spanned by the distinct points $\varphi(z)$ and $\varphi(w)$ on the curve $C$ is given by the row space of the matrix

$$
\left[\begin{array}{cccc}
z & z^{2}+z^{3} f(z) & z^{2} g(z) & 1 \\
w & w^{2}+w^{3} f(w) & w^{2} g(w) & 1
\end{array}\right]
$$

The dual Plücker coordinates are skew-symmetric power series, so Lemma 5.34 implies that they are divisible by $z-w$. In particular, if $f(z)=\sum_{i} a_{i} z^{i}$, then we have $q_{03}=z-w$,

$$
\begin{aligned}
& q_{01}=z\left(w^{2}+w^{3} f(w)\right)-w\left(z^{2}+z^{3} f(z)\right)=-z w(z-w)\left(1+\sum_{i} a_{i} \sum_{j=0}^{i+1} w^{j} z^{i+1-j}\right) \\
& q_{13}=z^{2}+z^{3} f(z)-w^{2}-w^{3} f(w)=(z-w)\left(z+w+\sum_{i} a_{i} \sum_{j=0}^{i+2} z^{j} w^{i+2-j}\right) .
\end{aligned}
$$


The symmetric square $\left(\mathbb{A}^{1}\right)^{(2)}$ of the affine line $\mathbb{A}^{1}$ is a smooth surface isomorphic to the affine plane $\mathbb{A}^{2}$; see [49, Example 10.23]. Consider the map $\mathcal{\omega}:\left(\mathbb{A}^{1}\right)^{(2)} \rightarrow \operatorname{Sec}(C)$ defined by sending the pair $\{z, w\}$ of points in $\mathbb{A}^{1}$ to the line spanned by the points $\varphi(z)$ and $\varphi(w)$ if $z \neq w$ or to $\mathbb{T}_{C, \varphi(z)}$ if $z=w$. In other words, the map $\omega$ sends $\{z, w\}$ to

$$
\begin{array}{r}
\left(-z w+h_{1}(z, w): \frac{q_{02}}{z-w}: 1: \frac{q_{12}}{z-w}: z+w+h_{2}(z, w): \frac{q_{23}}{z-w}\right), \text { where } \\
h_{1}(z, w):=-z w \sum_{i} a_{i} \sum_{j=0}^{i+1} w^{j} z^{i-j+1} \quad \text { and } \quad h_{2}(z, w):=\sum_{i} a_{i} \sum_{j=0}^{i+2} z^{j} w^{i+2-j} .
\end{array}
$$

Since the forms $z w$ and $z+w$ are local coordinates of $\left(\mathbb{A}^{1}\right)^{(2)}$ in a neighborhood of the origin, we conclude that $\omega$ is a local isomorphism and $\operatorname{Sec}(C)$ is smooth at the point corresponding to $L$.

Suppose the line $L$ intersects the curve $C$ at the point $v$ with multiplicity at least three. It follows that the line $L$ is contained in the Zariski closure of the set of lines that intersect $C$ in at least three points or that intersect $C$ in two points, one with multiplicity at least two. By Propositions 5.32 and 5.33 , we conclude that the line is singular in $\operatorname{Sec}(C)$.

Corollary 5.36. Let $C$ be a nondegenerate smooth irreducible curve in $\mathbb{P}^{3}$. If the line $L$ corresponds to a point in $\operatorname{Sec}(C)$, then $L$ corresponds to a singular point of $\operatorname{Sec}(C)$ if and only if one of the following three conditions is satisfied:

- the line L intersects the curve $C$ in three or more distinct points,

- the line L intersects the curve $C$ in exactly two points and $L$ is the tangent line to one of these two points,

- the line L intersects the curve $C$ at a single point with multiplicity at least three.

Proof. Combine Propositions 5.32 and 5.33, and Theorem 5.35.

Analogously, we want to describe the singularities of the inflectional locus $\operatorname{Infl}(S)$ and the bitangent locus $\operatorname{Bit}(S)$ of a surface $S \subset \mathbb{P}^{3}$.

Theorem 5.37. If $S \subset \mathbb{P}^{3}$ is an irreducible smooth surface of degree at least four which does not contain any lines, then the singular locus of $\operatorname{Infl}(S)$ corresponds to lines which either intersect $S$ with multiplicity at least three at two or more distinct points, or intersect $S$ with multiplicity at least four at some point.

Proof. We consider the incidence variety

$$
\Psi_{S}:=\overline{\{(v, L) \mid L \text { intersects } S \text { at } v \text { with multiplicity } 3\}} \subset S \times \operatorname{Gr}\left(1, \mathbb{P}^{3}\right) .
$$

The projection $\pi: \Psi_{S} \rightarrow \operatorname{Infl}(S)$, defined by $(v, L) \mapsto L$, is a surjective morphism. Since $S$ does not contain lines, all fibers of $\pi$ are finite and Lemma 5.6 implies that the map $\pi$ is finite. Moreover, the general fiber of $\pi$ has cardinality 
one, so $\pi$ is birational. To apply Lemma 5.3 , we need to examine the singularities of $\Psi_{S}$ and the differential of $\pi$.

Let $f$ be the defining equation for $S$ in $\mathbb{P}^{3}$ in the unknowns $x_{0}, \ldots, x_{3}$. We use dual Plücker coordinates $q_{01}, \ldots, q_{23}$ on $\operatorname{Gr}\left(1, \mathbb{P}^{3}\right)$. Consider the affine chart in $\mathbb{P}^{3} \times \operatorname{Gr}\left(1, \mathbb{P}^{3}\right)$ where $x_{0} \neq 0$ and $q_{01} \neq 0$. We may assume $v=(1: \alpha: \beta: \gamma)$ and the line $L$ is spanned by the points $(1: 0: a: b)$ and $(0: 1: c: d)$. In this affine chart, $S$ is defined by $g_{0}\left(x_{1}, x_{2}, x_{3}\right):=f\left(1, x_{1}, x_{2}, x_{3}\right)$. As in the proof of Theorem 5.8, we have $v \in L$ if and only if $a=\beta-\alpha c$ and $b=\gamma-\alpha d$. Parametrizing the line $L$ by $\ell(s, t):=(s: s \alpha+t: s \beta+t c: s \gamma+t d)$ for $(s: t) \in \mathbb{P}^{1}$ shows that $L$ intersects $S$ with multiplicity at least $m$ at $v$ if and only if $f(\ell(s, t))$ is divisible by $t^{m}$. This is equivalent to

$$
\left.\frac{\partial}{\partial t}[f(\ell(s, t))]\right|_{(1,0)}=\left.\frac{\partial^{2}}{\partial^{2} t}[f(\ell(s, t))]\right|_{(1,0)}=\cdots=\left.\frac{\partial^{m-1}}{\partial^{m-1} t}[f(\ell(s, t))]\right|_{(1,0)}=0 .
$$

Setting $g_{k}:=\left[\frac{\partial}{\partial x_{1}}+c \frac{\partial}{\partial x_{2}}+d \frac{\partial}{\partial x_{3}}\right]^{k} g_{0}$ for $k \geq 1$, the incidence variety $\Psi_{S}$ can be written on the chosen affine chart as

$$
\left\{(\alpha, \beta, \gamma, a, b, c, d) \mid g_{k}(\alpha, \beta, \gamma)=0 \text { for } 0 \leq k \leq 2, a=\beta-\alpha c, b=\gamma-\alpha d\right\}
$$

As $\operatorname{dim} \Psi_{S}=2$, it is smooth at the point $(v, L)$ if and only if its tangent space has dimension two or, equivalently, its Jacobian matrix

$$
\left[\begin{array}{ccccccc}
\frac{\partial g_{0}}{\partial x_{1}}(\alpha, \beta, \gamma) & \frac{\partial g_{0}}{\partial x_{2}}(\alpha, \beta, \gamma) & \frac{\partial g_{0}}{\partial x_{3}}(\alpha, \beta, \gamma) & 0 & 0 & 0 & 0 \\
\frac{\partial g_{1}}{\partial x_{1}}(\alpha, \beta, \gamma) & \frac{\partial g_{1}}{\partial x_{2}}(\alpha, \beta, \gamma) & \frac{\partial g_{1}}{\partial x_{3}}(\alpha, \beta, \gamma) & 0 & 0 & \frac{\partial g_{0}}{\partial x_{2}}(\alpha, \beta, \gamma) & \frac{\partial g_{0}}{\partial x_{3}}(\alpha, \beta, \gamma) \\
\frac{\partial g_{2}}{\partial x_{1}}(\alpha, \beta, \gamma) & \frac{\partial g_{2}}{\partial x_{2}}(\alpha, \beta, \gamma) & \frac{\partial g_{2}}{\partial x_{3}}(\alpha, \beta, \gamma) & 0 & 0 & 2 \frac{\partial g_{1}}{\partial x_{2}}(\alpha, \beta, \gamma) & 2 \frac{\partial g_{1}}{\partial x_{3}}(\alpha, \beta, \gamma) \\
-c & 1 & 0 & -1 & 0 & -\alpha & 0 \\
-d & 0 & 1 & 0 & -1 & 0 & -\alpha
\end{array}\right]
$$

has rank five. Since $S$ is smooth, the first two and the last two rows of the Jacobian matrix are linearly independent. If $\Psi_{S}$ is singular at $(v, L)$, then the third row is a linear combination of the others; specifically, there exist scalars $\lambda, \mu$ such that $\frac{\partial g_{2}}{\partial x_{j}}(\alpha, \beta, \gamma)=\lambda \frac{\partial g_{1}}{\partial x_{j}}(\alpha, \beta, \gamma)+\mu \frac{\partial g_{0}}{\partial x_{j}}(\alpha, \beta, \gamma)$ for $1 \leq j \leq 3$. It follows that $g_{3}(\alpha, \beta, \gamma)=\lambda g_{2}(\alpha, \beta, \gamma)+\mu g_{1}(\alpha, \beta, \gamma)=0$. Thus, the line $L$ intersects the surface $S$ at the point $v$ with multiplicity at least four if $\Psi_{S}$ is singular at $(v, L)$.

It remains to show that the differential $D_{(v, L)} \pi$ is not injective if and only if the line $L$ intersects the surface $S$ at the point $v$ with multiplicity at least four. The differential $D_{(v, L)} \pi$ sends every element in the kernel of the Jacobian matrix to its last four coordinates. This map is not injective if and only if the kernel contains an element of the form $(*, *, *, 0,0,0,0) \neq 0$. Such an element belongs to the kernel if and only if it equals $(\lambda, c \lambda, d \lambda, 0,0,0,0)$ for some non-zero constant $\lambda$ and $g_{1}(\alpha, \beta, \gamma)=g_{2}(\alpha, \beta, \gamma)=g_{3}(\alpha, \beta, \gamma)=0$. This shows that the line $L$ intersects the surface $S$ at the point $v$ with multiplicity at least four if and only if $D_{(v, L)}$ is not injective. 
Finally, the fiber $\pi^{-1}(L)$ consists of more than one point if and only if $L$ intersects $S$ with multiplicity at least three at two or more distinct points, so Lemma 5.3 completes the proof.

Proof of Theorem 5.1. The first part related to the curve $C$ is an amalgamation of Theorems 5.8 and 5.10, and Corollary 5.36. Similarly, the second part related to the surface $S$ follows from Theorems 5.13, 5.18 and 5.37 .

Remark 5.38. Analogously to Remark 5.14, we can formulate Theorem 5.37 for surfaces which contain at most finitely many lines. So let $S \subset \mathbb{P}^{3}$ be an irreducible smooth surface of degree at least three which contains finitely many lines $L_{1}, \ldots, L_{k}$. For a line $L \notin\left\{L_{1}, \ldots, L_{k}\right\}$, we have that $L$ corresponds to a singular point of $\operatorname{Infl}(S)$ if and only if $L$ is either inflectional at at least two points of $S$ or it intersects $S$ with multiplicity at least four at some point. The proof of this assertion is completely analogous to the proof of Theorem 5.37, except that we have to remove the lines $L_{1}, \ldots, L_{k}$ from the incidence variety (5.3); cf. Remark 5.14

Proposition 5.39. Let $S \subset \mathbb{P}^{3}$ be a general irreducible surface of degree at least four. If $L$ is a line that is tangent to $S$ at three or more distinct points, then the line L corresponds to a singular point of $\operatorname{Bit}(S)$.

Proof. As in the proof of Proposition 5.32, the symmetric square $S^{(2)}$ is the quotient of $S \times S$ by the action of the symmetric group $\mathfrak{S}_{2}$. The projection $\omega$ from

$\overline{\left\{(\{x, y\}, L) \mid x, y \in \operatorname{Reg}(S), x \neq y, \quad x, y \in L \subset \mathbb{T}_{S, x} \cap \mathbb{T}_{S, y}\right\}} \subset S^{(2)} \times \operatorname{Gr}\left(1, \mathbb{P}^{3}\right)$

onto $\operatorname{Bit}(S)$, defined by sending the pair $(\{x, y\}, L) \mapsto L$ is a birational morphism. The fiber $\mathscr{\omega}^{-1}(L)$ is a finite set containing more than one element if $L$ is tangent to $S$ in at least three distinct points. Hence, $\omega^{-1}(L)$ is not connected and the Zariski Connectedness Theorem proves that $\operatorname{Bit}(S)$ is singular at $L$.

We do not yet have a full understanding of points in $\operatorname{Bit}(S)$ for which the corresponding lines have an intersection multiplicity greater than four at a point of $S$. We know that a line $L$ that is tangent to the surface $S$ at exactly two points corresponds to a smooth point in $\operatorname{Bit}(S)$ if and only if the intersection multiplicity of $L$ and $S$ at both points is exactly two. Moreover, given a line $L$ that is tangent to $S$ at a single point, the intersection multiplicity of $L$ and $S$ at this point is at least four, and the line $L$ corresponds to a smooth point of $\operatorname{Bit}(S)$ when the multiplicity is exactly four; see [5, Lem. 4.3]. To complete this picture, we make the following prediction.

Conjecture 5.40. Let $S \subset \mathbb{P}^{3}$ be a general irreducible surface of degree at least four. If a point in the bitangent congruence $\operatorname{Bit}(S)$ corresponds to a line $L$ that is tangent to $S$ at a single point $x$ and the intersection multiplicity of $L$ and $S$ at $x$ is at least five, then $L$ corresponds to a singular point of $\operatorname{Bit}(S)$. 
Even for a surface $S$ of degree five we do not know how to prove this. The reason for this is the following. There are in principle two possibilities on how to approximate the line $L$ by lines in $\operatorname{Bit}(S)$ with lower intersection multiplicities. Either $L$ is in the closure of a set of lines that have a double and a triple point in the intersection with $S$ (these are singular in $\operatorname{Bit}(S)$ by [5, Lem. 4.3]), or $L$ is in the closure of a set of lines that have two double and one single point in the intersection with $S$. The problem is that the last type of lines is smooth in $\operatorname{Bit}(S)$ by [5, Lem. 4.3], and thus we cannot conclude that $L$ is singular.

Conjecture 5.41. More generally, we conjecture that the iterated singular loci of the Hurwitz hypersurface of a general hypersurface $X \subset \mathbb{P}^{n}$ have the same combinatorial structure as the singular loci of coincident root loci [70].

\subsection{Tangent Spaces to Congruences}

In this section, we explicitly compute tangent spaces to the secant, inflectional and bitangent congruences. This will be useful in Chapter 6, where we investigate the irreducible components of the visual event surface of a given curve or surface in $\mathbb{P}^{3}$. We begin with an explicit description of the tangent spaces of secant congruences. This involves the osculating planes to a space curve, which will appear frequently in the subsequent chapters. At a smooth point $x$ of a curve $C$ in $\mathbb{P}^{3}$ there is a pencil of planes tangent to $C$ at $x$. Among those there is a unique plane which meets $C$ at $x$ with multiplicity at least three, called the osculating plane of $C$ at $x$.

Proposition 5.42. Let $\mathrm{C}$ be a nondegenerate smooth irreducible curve in $\mathbb{P}^{3}$, and let the line $L \subset \mathbb{P}^{3}$ correspond to a smooth point of $\operatorname{Sec}(C)$. If $L$ intersects $C$ at two distinct points $x_{1}$ and $x_{2}$, then

$$
T_{\operatorname{Sec}(C), L}=\left\{\varphi \in \operatorname{Hom}\left(\boldsymbol{L}, \mathbb{A}^{4} / \boldsymbol{L}\right) \mid \forall i \in\{1,2\}: \varphi\left(\boldsymbol{x}_{\boldsymbol{i}}\right) \subset\left(\mathbb{T}_{\boldsymbol{C}, \boldsymbol{x}_{\boldsymbol{i}}}+\boldsymbol{L}\right) / \boldsymbol{L}\right\} .
$$

If $L$ is the tangent line to $C$ at some point $p$ with osculating plane $H$, then

$$
T_{\operatorname{Sec}(C), L}=\left\{\varphi \in \operatorname{Hom}\left(\boldsymbol{L}, \mathbb{A}^{4} / \boldsymbol{L}\right) \mid \operatorname{im} \varphi \subset \boldsymbol{H} / \boldsymbol{L}\right\} .
$$

Proof. The first part (5.4) follows immediately from Proposition 3.10 resp. (3.3). For the second part (5.5), we choose coordinates such that $p=(1: 0: 0: 0)$, and $L=\mathbb{T}_{C, p}$ is the zero locus $L=Z\left(x_{2}, x_{3}\right)$. Without loss of generality, we may work in the affine open subset $\mathbb{P}^{3} \backslash Z\left(x_{0}\right)$. Since $C$ is smooth, there is a local analytic isomorphism $f$ from a neighborhood of the origin in $\mathbb{A}^{1}$ to a neighborhood of the point $p$ in $C$. The map $f$ has the form $f(t)=\left(f_{1}(t), f_{2}(t), f_{3}(t)\right)$ for some $f_{1}, f_{2}, f_{3} \in \mathbb{C} \llbracket t \rrbracket$ with $f(0)=(0,0,0)$. For all $\varepsilon \in \mathbb{C}$ with $f_{1}(\varepsilon) \neq 0$, we define

$$
f_{\varepsilon}(t):=\frac{1}{f_{1}(\varepsilon)}\left(\varepsilon f_{1}^{\prime}(0) f_{1}(t), f_{1}(\varepsilon) f_{2}(t)-f_{2}(\varepsilon) f_{1}(t), f_{1}(\varepsilon) f_{3}(t)-f_{3}(\varepsilon) f_{1}(t)\right) .
$$


Due to $L=\mathbb{T}_{C, p}$, we have $f_{1}^{\prime}(0) \neq 0$ and $f_{2}^{\prime}(0)=f_{3}^{\prime}(0)=0$. This implies that $\lim _{\varepsilon \rightarrow 0} f_{\varepsilon}(t)=f(t)$. Moreover, the line $L$ intersects the curve $C_{\varepsilon}$ parametrized by $f_{\varepsilon}$ at $f_{\varepsilon}(0)=(0,0,0)$ and $f_{\varepsilon}(\varepsilon)=\left(\varepsilon f_{1}^{\prime}(0), 0,0\right)$. For small enough $\varepsilon$, the line $L$ corresponds to a smooth point of the secant congruence of $C_{\varepsilon}$. Hence, we can apply (5.4) and consider the limit $\varepsilon \rightarrow 0$ to compute $T_{\operatorname{Sec}(C), L}$.

For $\delta \in\{0, \varepsilon\}$, the plane spanned by $L$ and the tangent line to $C_{\varepsilon}$ at $f_{\varepsilon}(\delta)$ is spanned by $(0,0,0),(1,0,0)$ and $\nabla f_{\mathcal{E}}(\delta)$, where

$$
\begin{aligned}
& \nabla f_{\varepsilon}(0)=\frac{f_{1}^{\prime}(0)}{f_{1}(\varepsilon)}\left(\varepsilon f_{1}^{\prime}(0),-f_{2}(\varepsilon),-f_{3}(\varepsilon)\right) \quad \text { and } \\
& \nabla f_{\varepsilon}(\varepsilon)=\frac{1}{f_{1}(\varepsilon)}\left(\varepsilon f_{1}^{\prime}(0) f_{1}^{\prime}(\varepsilon), f_{1}(\varepsilon) f_{2}^{\prime}(\varepsilon)-f_{2}(\varepsilon) f_{1}^{\prime}(\varepsilon), f_{1}(\varepsilon) f_{3}^{\prime}(\varepsilon)-f_{3}(\varepsilon) f_{1}^{\prime}(\varepsilon)\right) .
\end{aligned}
$$

Thus, by (5.4), the tangent space of $\operatorname{Sec}\left(C_{\varepsilon}\right)$ at $L$ is spanned by two homomorphisms $\varphi_{1}^{(\varepsilon)}, \varphi_{2}^{(\varepsilon)}: \boldsymbol{L} \rightarrow \mathbb{A}^{4} / \boldsymbol{L}$, where

$$
\begin{aligned}
\varphi_{1}^{(\varepsilon)}(1,0,0,0) & :=\left(0,0, f_{2}(\varepsilon), f_{3}(\varepsilon)\right)+L \\
\varphi_{1}^{(\varepsilon)}(0,1,0,0) & :=0+L \\
\text { and } & \\
\varphi_{2}^{(\varepsilon)}(1,0,0,0) & :=0+L \\
\varphi_{2}^{(\varepsilon)}(0,1,0,0) & :=\left(0,0, f_{1}(\varepsilon) f_{2}^{\prime}(\varepsilon)-f_{2}(\varepsilon) f_{1}^{\prime}(\varepsilon), f_{1}(\varepsilon) f_{3}^{\prime}(\varepsilon)-f_{3}(\varepsilon) f_{1}^{\prime}(\varepsilon)\right)+\boldsymbol{L} .
\end{aligned}
$$

Because of

$$
\lim _{\varepsilon \rightarrow 0} \frac{2}{\varepsilon^{2}} \varphi_{1}^{(\varepsilon)}(1,0,0,0)=\left(0,0, f_{2}^{\prime \prime}(0), f_{3}^{\prime \prime}(0)\right)+L=\lim _{\varepsilon \rightarrow 0} \frac{2}{\varepsilon^{2} f_{1}^{\prime}(0)} \varphi_{2}^{(\varepsilon)}(0,1,0,0),
$$

the tangent space $T_{\operatorname{Sec}(C), L}$ consists of all homomorphisms $L \rightarrow \mathbb{A}^{4} / L$ whose image is spanned by $\left(0,0, f_{2}^{\prime \prime}(0), f_{3}^{\prime \prime}(0)\right)+\boldsymbol{L}$. Since the osculating plane of $C$ at the point $(0,0,0)$ is spanned by $\left(f_{1}^{\prime}(0), 0,0\right)$ and $\left(f_{1}^{\prime \prime}(0), f_{2}^{\prime \prime}(0), f_{3}^{\prime \prime}(0)\right)$, we have proven (5.5).

We next focus on the tangent spaces of bitangent and inflectional congruences.

Lemma 5.43. Let $S$ be a surface in $\mathbb{P}^{3}$ of degree at least four, and let the line $L \subset \mathbb{P}^{3}$ correspond to a smooth point of $\operatorname{Bit}(S)$. If $L$ is tangent to $S$ at exactly two distinct points $x_{1}$ and $x_{2}$, then

$$
T_{\operatorname{Bit}(S), L}=\left\{\varphi \in \operatorname{Hom}\left(\boldsymbol{L}, \mathbb{A}^{4} / \boldsymbol{L}\right) \mid \varphi\left(\boldsymbol{x}_{\mathbf{1}}\right) \subset \mathbb{T}_{\boldsymbol{S}, \boldsymbol{x}_{\mathbf{1}}} / \boldsymbol{L}, \varphi\left(\boldsymbol{x}_{\mathbf{2}}\right) \subset \mathbb{T}_{\boldsymbol{S}, \boldsymbol{x}_{\mathbf{2}}} / \boldsymbol{L}\right\} .
$$

Proof. This follows immediately from Proposition 3.10 resp. (3.3).

Let $S \subset \mathbb{P}^{3}$ be a general surface of degree at least three. By Lemma 4.28 , a 
general point on $S$ has exactly two inflectional lines. There is a curve of those points on $S$ that have just one inflectional line. This is called the parabolic curve and is the intersection of $S$ and its Hessian surface $Z\left(\operatorname{det}\left(H_{f}\right)\right)$, where the entries of the Hessian matrix $H_{f}$ are the second partial derivatives of a defining polynomial $f$ of $S$.

Remark 5.44. As we have seen, a formal study of the singular loci of the families of lines described in this chapter presents many technical challenges. For example, in the course of examining inflectional lines at parabolic points of a surface $S$, we discovered a small error in [5, Sec. 4], where Arrondo et al. consider the incidence variety (5.3) for inflectional tangents of $S$. They denote it by $Y_{2}$. Lemma $4.1 \mathrm{~b})$ in [5] states that the surface $Y_{2}$ is singular at points $(x, L)$ for which $x$ is on the parabolic curve. This is incorrect. A general cubic surface $S$ has a parabolic curve of degree 12 . However, the incidence variety $Y_{2}$ is smooth. This is shown by direct computation; see Code $\mathrm{D}$ in the Appendix.

The inflectional line $L$ at a general parabolic point of $S$ corresponds to a smooth point of $\operatorname{Infl}(S)$; see Remark 5.38. By Theorem 4.26, the projectivized conormal space of $\operatorname{Infl}(S)$ at $L$ is a tangent line to the quadric $\operatorname{Seg}\left(\mathbb{A}^{4} / L, L\right)$. Now we show that it is in fact a line on one of the two rulings of this Segre variety. Moreover, projectivized conormal spaces to $\operatorname{Infl}(S)$ at lines $L$ which are inflectional at non-parabolic points of $S$ are tangent lines to the Segre variety that are not contained in any ruling.

Note that the Segre varieties we consider here are self-dual. More specifically, for two-dimensional vector spaces $U$ and $W$, we have $\operatorname{Seg}(U, W)^{\vee}=\operatorname{Seg}(W, U)$. In particular, a line is tangent to $\operatorname{Seg}(U, W)$ if and only its dual line is tangent to $\operatorname{Seg}(W, U)$. Furthermore, a line is contained in $\operatorname{Seg}(U, W)$ if and only if its dual line is contained in $\operatorname{Seg}(W, U)$.

Theorem 5.45. Let $S$ be a general surface in $\mathbb{P}^{3}$ of degree at least three, and let the line $L \subset \mathbb{P}^{3}$ correspond to a smooth point of $\operatorname{Infl}(S)$. If $\operatorname{deg} S=3$, we assume that $L$ is not one of the 27 lines on $S$.

1. If $L$ is an inflectional line at a point $p \in S$, the projectivization of $T_{\operatorname{Infl}(S), L}$ is a tangent line to the Segre variety $\operatorname{Seg}\left(L, \mathbb{A}^{4} / L\right)$ at the unique point $\varphi \in$ $\mathbb{P}\left(\operatorname{Hom}\left(\boldsymbol{L}, \mathbb{A}^{4} / \boldsymbol{L}\right)\right)$ with $\operatorname{ker} \varphi=\boldsymbol{p}$ and $\operatorname{im} \varphi=\mathbb{T}_{S, p} / \boldsymbol{L}$.

2. The point $p$ is parabolic if and only if every homomorphism in $T_{\operatorname{Infl}(S), L}$ has rank at most one. In that case, we have

$$
T_{\operatorname{Infl}(S), L}=\left\{\varphi \in \operatorname{Hom}\left(\boldsymbol{L}, \mathbb{A}^{4} / \boldsymbol{L}\right) \mid \operatorname{im}(\varphi) \subset \mathbb{T}_{S, p} / \boldsymbol{L}\right\}
$$

Proof. The first part is a special case of Theorem 4.26 for $m=3$. For the second part, we repeat the proof of Lemma 4.29 for the special case $m=3$ without assuming that $L \in \operatorname{Infl}(S)$ is general. Instead, we only assume $L$ to be a smooth point of $\operatorname{Infl}(S)$ and to be not contained in $S$. By Remark 5.38, the rational map $\operatorname{Infl}(S) \rightarrow S$ which sends a general $L^{\prime} \in \operatorname{Infl}(S)$ to the unique point $p_{L^{\prime}} \in S$ at 
which $L^{\prime}$ intersects $S$ with multiplicity three is defined on all of $\operatorname{Reg}(\operatorname{Infl}(S)) \backslash \mathcal{L}$, where $\mathcal{L}$ denotes the set of the 27 lines on $S$ if $S$ is a cubic surface and $\mathcal{L}=\varnothing$ if the degree of $S$ is at least four. We choose coordinates on $\mathbb{P}^{3}$ such that $p=Z\left(x_{1}, x_{2}, x_{3}\right), L=Z\left(x_{2}, x_{3}\right)$, and $\mathbb{T}_{S, p}=Z\left(x_{3}\right)$. Moreover, we work in the affine chart $\mathbb{P}^{3} \backslash Z\left(x_{0}\right)$ with the standard basis $e_{1}, e_{2}, e_{3}$, and we extend this basis to a basis for $\mathbb{A}^{4}$ by adding $e_{0} \in \boldsymbol{p} \backslash\{0\}$. We write $f(x)=f_{1}(x)+f_{2}(x)+\ldots$ for the defining polynomial of $S$ in this affine chart, where $f_{i}$ is homogeneous of degree $i$.

We first assume that $p$ is not parabolic. We will show that the differential $\Phi_{L}: T_{\operatorname{Infl}(S), L} \rightarrow T_{S, p}$ of the rational map $\operatorname{Infl}(S) \rightarrow S$ at $L$ is bijective, and we will compute $T_{\operatorname{Infl}(S), L}$ explicitly. By our choice of coordinates, we may assume $f_{1}(x)=x_{3}$ and $f_{2}(x)=x_{2} \ell_{1}(x)+x_{3} \ell_{2}(x)$, where $\ell_{1}$ and $\ell_{2}$ are homogeneous linear forms, and $\ell_{1} \notin\left\langle x_{2}, x_{3}\right\rangle$ since $p$ is not parabolic. Using the notation in (4.14), we have $c_{1,3}=1, c_{2,2}=\ell_{1}\left(e_{1}\right) \neq 0$, and $c_{2,3}=\ell_{2}\left(e_{1}\right)$. Now we follow exactly the remaining proof of Lemma 4.29 to compute the fibers of the tangent directions $e_{i}$ for $i \in\{1,2\}$ under $\Phi_{L}$.

$i=1$ : As in the proof of Lemma 4.29, solving (4.17) for $\left(d_{1}, d_{2}, d_{3}\right)$ implies $d_{3}=0$. Furthermore, by Remark 5.38 , the line $L$ has exactly intersection multiplicity three at $p$, which shows $f_{3}\left(e_{1}\right) \neq 0$ and $d_{2} \neq 0$. From 4.16 we see that $\Phi_{L}^{-1}\left(T_{L, p}\right)$ is spanned by

$$
\begin{aligned}
& \varphi_{1}: L \longrightarrow \mathbb{A}^{4} / \boldsymbol{L}, \\
& e_{0} \longmapsto 0+L, \\
& e_{1} \longmapsto e_{2}+\boldsymbol{L} .
\end{aligned}
$$

$i=2:$ As in the proof of Lemma 4.29, we solve the system

$$
\begin{aligned}
& F_{1}^{(2)}(v(t))=\left(d_{3}+\ell_{1}\left(e_{1}\right)\right) \cdot t+O\left(t^{2}\right) \\
& F_{2}^{(2)}(v(t))=\left(\ell_{1}\left(e_{1}\right) d_{2}+\ell_{2}\left(e_{1}\right) d_{3}+\frac{\partial f_{3}}{\partial x_{2}}\left(e_{1}\right)\right) \cdot t+O\left(t^{2}\right) .
\end{aligned}
$$

This yields $d_{3}=-\ell_{1}\left(e_{1}\right) \neq 0$ and $d_{2}=\ell_{2}\left(e_{1}\right)-\frac{1}{\ell_{1}\left(e_{1}\right)} \cdot \frac{\partial f_{3}}{\partial x_{2}}\left(e_{1}\right)$. From 4.16 we see that $\Phi_{L}^{-1}\left(T_{S, p}\right)=T_{\operatorname{Infl}(S), L}$ is spanned by $\varphi_{1}$ and

$$
\begin{aligned}
\varphi_{2}: L & \longrightarrow \mathbb{A}^{4} / \boldsymbol{L}, \\
e_{0} & \longmapsto e_{2}+\boldsymbol{L}, \\
e_{1} & \longmapsto-\ell_{1}\left(e_{1}\right) e_{3}+\boldsymbol{L} .
\end{aligned}
$$

In particular, the rank of $\varphi_{2}$ is two.

Finally, we assume that $p$ is a parabolic point of $S$. Due to our choice of coordinates, we may assume $f_{1}(x)=x_{3}$ and $f_{2}(x)=x_{2}^{2}+x_{3} \ell(x)$, where $\ell$ is a homogeneous linear form. Using the notation in (4.14), we have $c_{1,3}=1$, 
but $c_{2,2}=0$. Therefore, we cannot follow the lines of the proof of Lemma 4.29 directly. Instead we compute the tangent space $T_{\operatorname{Infl}(S), L}$ in this case as the limit of non-parabolic cases. More specifically, we change the defining equation $f$ to $f^{(\varepsilon)}=f_{1}^{(\varepsilon)}+f_{2}^{(\varepsilon)}+\ldots$, where $f_{i}^{(\varepsilon)}:=f_{i}$ if $i \neq 2$ and $f_{2}^{(\varepsilon)}:=x_{2}\left(x_{2}+\varepsilon x_{1}\right)+x_{3} \ell(x)$. For all $\varepsilon \neq 0$, the zero locus $L=Z\left(x_{2}, x_{3}\right)$ is still an inflectional line to the surface $S^{(\varepsilon)}$ defined by $f^{(\varepsilon)}$ at the origin of the affine chart, but now the origin is a nonparabolic point of $S^{(\varepsilon)}$. Hence, we can use our computations above to deduce that $T_{\operatorname{Infl}\left(S^{(\varepsilon)}\right), L}$ is spanned by $\varphi_{1}$ and

$$
\begin{aligned}
\varphi_{2}^{(\varepsilon)}: L & \longrightarrow \mathbb{A}^{4} / \boldsymbol{L}, \\
e_{0} & \longmapsto e_{2}+\boldsymbol{L}, \\
e_{1} & \longmapsto-\varepsilon e_{3}+\boldsymbol{L} .
\end{aligned}
$$

Taking the limit $\varepsilon \longrightarrow 0$ shows (5.7).

The congruences we have studied in this chapter, their singular loci and tangent spaces will appear again in the next chapter, where they play a crucial role in describing the irreducible components of the visual event surface of a given curve or surface in $\mathbb{P}^{3}$. 


\section{Changing Views on Curves and Surfaces}

Consider a curve or surface in three-dimensional space, and pretend you are taking a picture of that object with a camera. If the object is a curve, you see again a curve in the image plane. For a surface, you see a region bounded by a curve, which is called image contour or outline curve. The outline is the natural sketch one might use to depict the surface, and is the projection of the critical points where viewing lines are tangent to the surface. In both cases, the image curve has singularities that arise from the projection, even if the original curve or surface is smooth. Now, let your camera travel along a path in threedimensional space. This path naturally breaks up into segments according to how the picture looks like. Within each segment, the picture looks alike, meaning that the topology and singularities of the image curve do not change.

The appearance of a solid object under a continuously varying viewpoint was studied in the 1970s by Koenderink and van Doorn [63]. Their motivation came from visual perception in psychology and artificial intelligence. Koenderink offers a detailed discussion in his remarkable book on Solid Shape [62]. On the mathematical side, the topic was studied in singularity theory by Arnol'd and others [3, 59, 83]. In that setting, the transitions between locally stable views are the non-generic singularities from catastrophe theory. These catastrophes have been classified for projection-generic surfaces. The catalogue consists of the following six visual events. The first three names are due to René Thom [104]:

(L) Local events: lip, beak-to-beak, and swallowtail.

(M) Multi-local events: tangent crossing, cusp crossing, and triple point.

In the 1980s, visual events became a research topic in computer vision [10, 76, 78, 85]. Chapter 13 in the textbook by Forsyth and Ponce [38] offers an introduction in that context. The motivation in computer vision was to give a description of all possible appearances of a solid object using a finite number of stable views, or aspects. The overall structure of aspects and events is encoded in the aspect graph, in which vertices correspond to aspects, and edges correspond to visual events between stable views. Although these ideas never found much practical use, several algorithms for computing aspect graphs of algebraic surfaces were proposed. Test implementations involved both numerical and symbolic methods. Ponce and Kriegman [85] and Rieger [90] studied the case of orthographic projections of parametric algebraic surfaces. Methods for implicit algebraic surfaces were introduced by Petitjean et al. [78] for orthographic projections, and by 
Rieger [91] for perspective projections. All examples shown in these articles are very special low-degree surfaces. We here revisit this literature, now 25 years old, and develop it further for today's applied algebraic geometry.

Our model for the object to be viewed is a smooth variety $X$ of dimension one or two in complex projective space $\mathbb{P}^{3}$. We assume that $X$ is defined over $\mathbb{R}$ and the real locus $X_{\mathbb{R}}$ is Zariski dense in $X$. Taking a picture of $X_{\mathbb{R}}$ is modeled by the linear projection $\pi_{z}: \mathbb{P}^{3} \rightarrow \mathbb{P}^{2}$ with center $z$ (for Zentrum). This defines a curve $C_{z}(X)$ in the image plane $\mathbb{P}^{2}$. If $X$ is a curve, then $C_{z}(X)$ is the Zariski closure of the image of $X$ under $\pi_{z}$. If $X$ is a surface, then $C_{z}(X)$ is the branch locus of $\pi$ restricted to $X$. This is the Zariski closure of the set of points in $\mathbb{P}^{2}$ whose corresponding viewing lines are tangent to $X$. Even though $X$ is smooth, the curve $C_{z}(X)$ has many singular points. For a surface viewed from a general viewpoint $z$, the only singularities in the contour are nodes and cusps. For a space curve, the image curve has only nodes. As the center $z$ changes, the structure of its singularities is locally constant. At some point, a transition occurs, and the singularity structure changes. The visual event surface $\mathcal{V}(X)$ is the Zariski closure in $\mathbb{P}^{3}$ of the set of these transition points. This definition can be extended to singular curves and surfaces by excluding the role of singular points on $X$.

The visual event surface $\mathcal{V}(X)$ is usually reducible. If $X$ is a general curve, then $\mathcal{V}(X)$ has three irreducible components. If $X$ is a general surface, then $\mathcal{V}(X)$ has five irreducible components. These arise from the six events in (L) and $(\mathrm{M})$ above. We shall explain the geometry of these irreducible components and their parametrization by the iterated singular loci of the coisotropic hypersurfaces of $X$. In addition, we discuss how to compute them in practice. An important caveat for applications is the distinction between real and complex points. Algebraic methods do not distinguish between them. They apply to any complex curve or surface $X$ in $\mathbb{P}^{3}$. For any particular $X$ that is defined over $\mathbb{R}$, it can happen that some visual events are not seen on its real points, i.e., they might live in the set $X \backslash X_{\mathbb{R}}$ of complex points.

The focus in this chapter lies on curves and surfaces that are general in the sense of algebraic geometry. Thus, for a surface $X$ in $\mathbb{P}^{3}$ of degree $d$, we assume that its defining equation is general among homogeneous polynomials of degree $d$ in four variables. For a curve $X$ in $\mathbb{P}^{3}$ of degree $d$ and genus $g$, we assume that it is a general point in the Hilbert scheme of such curves.

We now briefly describe the organization and main results in this chapter. Section 6.1 is devoted to ruled surfaces in $\mathbb{P}^{3}$ and to its subclass of developable surfaces. We introduce effective representations of ruled surfaces, and we show how to compute with these. This is relevant because all visual event surfaces are ruled. Their irreducible components are the ruled surfaces in the bottom rows of Figures 6.2 and 6.3. These arise as iterated singular loci of Chow and Hurwitz threefolds in $\operatorname{Gr}\left(1, \mathbb{P}^{3}\right)$, and of dual varieties in $\left(\mathbb{P}^{3}\right)^{*}$.

In Section 6.2, we develop the geometry of visual event surfaces for curves in $\mathbb{P}^{3}$. The three irreducible components are the tangential surface, edge surface and trisecant surface. These represent the three Reidemeister moves on 
the image curve, as shown in Figure 6.4. We demonstrate how the coisotropic hypersurfaces of the given space curve can be used to prove that these are all irreducible components. Finally, we present case studies that show the computation of visual event surfaces for curves up to degree six.

Section 6.3 concerns the visual event surface $\mathcal{V}(X)$ of a general surface $X$ in $\mathbb{P}^{3}$. The six events in $(\mathrm{L})$ and $(\mathrm{M})$ are depicted in Figure 6.5, which we discuss in detail. These events are translated into the algebraic setting, where they correspond to the five irreducible components of $\mathcal{V}(X)$. We use again the coisotropic hypersurfaces of the given surface to derive these five irreducible components. Their degrees are listed in Theorem 6.19. These formulas were known classically: they appear in paragraphs 597, 598, 599, 608 and 613 of Salmon's book [94]. Modern proofs were given by Petitjean [77]. In Section 6.4. we present new proofs, based on intersection theory in algebraic geometry, as seen in the textbook by Eisenbud and Harris [34].

Section 6.5 is devoted to practical methods for computing and representing the visual events associated with a surface $X$ in $\mathbb{P}^{3}$. This is a non-trivial matter because the degrees of the ruled surfaces in the output are very high, as seen in Table 6.2. For instance, if $X$ is a quintic, then the degrees of the irreducible components of $\mathcal{V}(X)$ range between 260 and 930.

\subsection{Ruled Surfaces and Developable Surfaces}

An irreducible surface in $\mathbb{P}^{3}$ is ruled if it is covered by straight lines. These lines are parametrized by some curve $C$, and they are known as the generators of the surface. A first example are smooth quadratic surfaces in $\mathbb{P}^{3}$. These possess two rulings of lines over $\mathbb{C}$. We refer to the book by Edge [33] for many classical results on ruled surfaces. In this section, we develop algebraic tools for computing and representing ruled surfaces in practice.

Ruled surfaces arise naturally when taking pictures of an object in threedimensional space. We encounter them because all components of a visual event surface $\mathcal{V}(X)$ are ruled. Indeed, every general point $z$ on $\mathcal{V}(X)$ determines a line of sight that has a special intersection with the curve or surface $X$. Every point on the line shares this property with $z$ and hence lies in $\mathcal{V}(X)$.

Consider now an irreducible curve $C$ in $\operatorname{Gr}\left(1, \mathbb{P}^{3}\right)$ whose image under the Plücker embedding has degree $d$ in $\mathbb{P}^{5}$. We write $I_{C}$ for its prime ideal in the coordinate ring of $\operatorname{Gr}\left(1, \mathbb{P}^{3}\right)$. The union of all lines on $C$ is a ruled surface $\mathcal{S}_{C}$ in $\mathbb{P}^{3}$.

Lemma 6.1. The ruled surface $\mathcal{S}_{C}$ is irreducible and it has degree in $\mathbb{P}^{3}$. Conversely, every irreducible ruled surface in $\mathbb{P}^{3}$ arises in this way from some irreducible curve $C \subset \operatorname{Gr}\left(1, \mathbb{P}^{3}\right)$.

Proof. This is one of the basic facts derived in Edge's book [33, Ch. I, §26].

The defining polynomial of the surface $\mathcal{S}_{C} \subset \mathbb{P}^{3}$ can be computed from the 
equations

$$
\left[\begin{array}{cccc}
0 & p_{01} & p_{02} & p_{03} \\
-p_{01} & 0 & p_{12} & p_{13} \\
-p_{02} & -p_{12} & 0 & p_{23} \\
-p_{03} & -p_{13} & -p_{23} & 0
\end{array}\right] \cdot\left(\begin{array}{l}
x_{0} \\
x_{1} \\
x_{2} \\
x_{3}
\end{array}\right)=\left(\begin{array}{l}
0 \\
0 \\
0 \\
0
\end{array}\right)
$$

where the $p_{i j}$ are primal Plücker coordinates. We add these four bilinear forms to the ideal $I_{C}$, and then we saturate with respect to the irrelevant ideal $\left\langle p_{01}, p_{02}, p_{03}, p_{12}, p_{13}, p_{23}\right\rangle$ of $\mathbb{P}^{5}$. The resulting ideal is prime, and it describes the incidence correspondence of points on lines that are in the curve $C$. Now, by eliminating the unknowns $p_{i j}$, we obtain a principal homogeneous prime ideal in $\mathbb{C}\left[x_{0}, x_{1}, x_{2}, x_{3}\right]$. The generator of this ideal is the polynomial of degree $d$ that defines the desired surface.

This computation can be reversed. Given a surface $\mathcal{S}$ in $\mathbb{P}^{3}$, we can compute the Fano scheme of all lines on $\mathcal{S}$. This lives in $\operatorname{Gr}\left(1, \mathbb{P}^{3}\right)$. To obtain its ideal in $\mathbb{C}\left[p_{01}, p_{02}, p_{03}, p_{12}, p_{13}, p_{23}\right]$ we use that every line in $\mathbb{P}^{3}$ satisfying $p_{23} \neq 0$ has the parametric representation

$$
z(t)=\left(-p_{23}: t p_{23}: p_{03}-t p_{13}: t p_{12}-p_{02}\right) \text {. }
$$

We substitute (6.2) into the equation of $\mathcal{S}$, extract the coefficients of the resulting polynomial in $t$, and saturate their ideal by $\left\langle p_{23}\right\rangle$. The Fano scheme is usually empty or consists of points. However, if it is a curve $C$, then the surface is ruled and $\mathcal{S}=\mathcal{S}_{C}$.

Typically, the dual $S^{\vee}$ of an irreducible surface $S$ of degree at least two will be a surface in $\left(\mathbb{P}^{3}\right)^{*}$. However, it can happen that $S^{\vee}$ is a curve. In that case, $S$ is called developable. Each developable surface $\mathcal{S}$ is encoded by its dual curve $\mathcal{S}^{\vee}$ since we can recover the surface by the biduality relation $\mathcal{S}=\left(\mathcal{S}^{\vee}\right)^{\vee}$.

Theorem 6.2. Every developable surface $\mathcal{S}$ is a ruled surface, i.e., it satisfies $\mathcal{S}=\mathcal{S}_{C}$ for some curve $C$ in $\operatorname{Gr}\left(1, \mathbb{P}^{3}\right)$. For a curve $C$ in $\operatorname{Gr}\left(1, \mathbb{P}^{3}\right)$, the corresponding ruled surface $\mathcal{S}_{C}$ is developable if and only if all tangent lines of the image of $C$ under the Plücker embedding into $\mathbb{P}^{5}$ are contained in the Plücker quadric.

Proof. For the first statement see [33, Ch. V, §344]. The second is [7, Prop. 12.4.1]. We give an alternative derivation for the second assertion in Example 7.11.

A developable surface $\mathcal{S}=\mathcal{S}_{C}$ that is not a cone has three distinct encodings as a curve. First, there is the curve $C$ in the Grassmannian $\operatorname{Gr}\left(1, \mathbb{P}^{3}\right)$. Second, there is the dual curve $\mathcal{S}^{\vee}$ in $\left(\mathbb{P}^{3}\right)^{*}$. We saw how to recover $\mathcal{S}$ from these encodings. Finally, there is the edge of regression $E(\mathcal{S})$ which lies on the surface $\mathcal{S}$ in $\mathbb{P}^{3}$. Points in $E(\mathcal{S})$ are the osculating planes to the curve $\mathcal{S}^{\vee}$. The surface $\mathcal{S}$ is the tangential surface of $E(\mathcal{S})$, i.e., it is the union of lines that are tangent to $E(\mathcal{S})$ (see [82, page 111]). This also verifies that $\mathcal{S}$ is indeed a ruled surface. The curves $E(\mathcal{S})$ and $\mathcal{S}^{\vee}$ are also related by a biduality relation; namely, $\mathcal{S}^{\vee}$ is the curve consisting of the osculating planes of $E(\mathcal{S})$ [79, Thm. 5.1]. Moreover, the tangent lines of $E(\mathcal{S})$ and $\mathcal{S}^{\vee}$ are dual to each other. We summarize these duality relations in Figure 6.1. where $\mathcal{T}(X) \subset \mathbb{P}^{3}$ denotes the tangential surface of a 


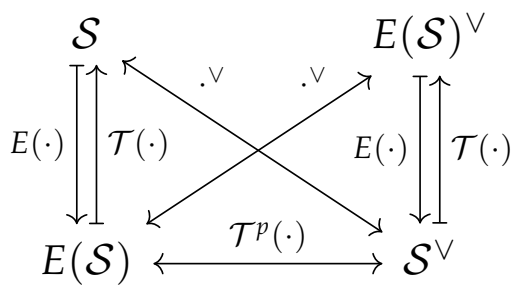

Figure 6.1: Duality relations of a developable surface $\mathcal{S}$.

curve $X \subset \mathbb{P}^{3}$ and $\mathcal{T}^{p}(X) \subset\left(\mathbb{P}^{3}\right)^{*}$ denotes the curve formed by its osculating planes. This situation degenerates when the surface $\mathcal{S}$ is a cone, which means that its dual $\mathcal{S}^{\vee}$ is a plane curve. In that special case, the edge of regression $E(\mathcal{S})$ is the vertex of the cone $\mathcal{S}$.

We illustrate the three curve encodings of a developable surface with a simple example.

Example 6.3. The Macaulay2 code for the following computations is listed in Code $\mathrm{E}$ in the Appendix. Let $\mathcal{S}$ be the surface of degree six in $\mathbb{P}^{3}$ that is defined by the polynomial

$$
f=16 x_{1}^{3} x_{2}^{3}-27 x_{0}^{2} x_{2}^{4}+6 x_{0} x_{1}^{2} x_{2}^{2} x_{3}-27 x_{1}^{4} x_{3}^{2}+48 x_{0}^{2} x_{1} x_{2} x_{3}^{2}-16 x_{0}^{3} x_{3}^{3} .
$$

This is the surface in [97, $\S 3$, eqn. (9)]. We verify that $\mathcal{S}$ is developable by computing the ideal of its dual variety $\mathcal{S}^{\vee} \subset\left(\mathbb{P}^{3}\right)^{*}$. This shows that $\mathcal{S}^{\vee}$ is a smooth rational quartic curve defined by

$$
\left\langle y_{1} y_{2}-4 y_{0} y_{3}, y_{2}^{3}+4 y_{1} y_{3}^{2}, y_{0} y_{2}^{2}+y_{1}^{2} y_{3}, y_{1}^{3}+4 y_{0}^{2} y_{2}\right\rangle
$$

The curve $C$ in the Grassmannian $\operatorname{Gr}\left(1, \mathbb{P}^{3}\right)$ that encodes the ruling of $\mathcal{S}=\mathcal{S}_{C}$ has the ideal

$$
\left\langle 2 p_{03}-p_{12}, p_{13}^{2}+3 p_{02} p_{23}, p_{02} p_{13}-9 p_{01} p_{23}, p_{12}^{2}-16 p_{01} p_{23}, p_{02}^{2}+3 p_{01} p_{13}\right\rangle .
$$

This ideal defines the Fano scheme of $\mathcal{S}$ in $\mathbb{P}^{5}$. Finally, the edge of regression $E(\mathcal{S})$ is the rational quartic curve $\left\{\left(s^{4}: s^{3} t: s t^{3}: t^{4}\right)\right\}$ in $\mathbb{P}^{3}$. The ideal of this curve equals

$$
\left\langle x_{1} x_{2}-x_{0} x_{3}, x_{2}^{3}-x_{1} x_{3}^{2}, x_{0} x_{2}^{2}-x_{1}^{2} x_{3}, x_{1}^{3}-x_{0}^{2} x_{2}\right\rangle .
$$

This curve has $\mathcal{S}$ as its tangential surface. Note that (6.3) is isomorphic to (6.4). This reflects the isomorphism between (9) and (10) in [97]. All of these computations can be reversed. This example shows how various objects can serve as a representation of the surface $\mathcal{S}$.

Many of the ruled surfaces $\mathcal{S}_{C}$ we shall encounter in later sections have the property that their defining polynomial $f$ is extremely large and impossible to compute symbolically. In such cases, the curve $C$ in $\operatorname{Gr}\left(1, \mathbb{P}^{3}\right) \subset \mathbb{P}^{5}$ is more 
manageable, and we can often compute generators for its ideal $I_{C}$. This encoding of the ruling enables us to carry out computations with the surface $\mathcal{S}_{C}$. For example, suppose $\mathcal{S}_{C}$ has degree $d$ and consider a general line $L$ in $\mathbb{P}^{3}$. We may wish to compute the $d$ points in the intersection $\mathcal{S}_{C} \cap L$. This problem arises in our computer vision application when the camera travels along $L$. The real intersection points with the visual event surfaces are precisely the visual events we are interested in. by

Fix two points $\left(a_{0}: a_{1}: a_{2}: a_{3}\right)$ and $\left(b_{0}: b_{1}: b_{2}: b_{3}\right)$ on $L$, and parametrize $L$

$$
x_{i}=s a_{i}+t b_{i} \text { for } i=0,1,2,3 .
$$

To compute $\mathcal{S}_{C} \cap L$ from $I_{C}$, we substitute (6.5) into (6.1), we add the resulting four bilinear forms to $I_{C}$, we saturate with respect to $\left\langle p_{01}, \ldots, p_{23}\right\rangle$, and we then eliminate the six Plücker coordinates. The result is the principal ideal in $\mathbb{C}[s, t]$ that is generated by the binary form

$$
f\left(s a_{0}+t b_{0}, s a_{1}+t b_{1}, s a_{2}+t b_{2}, s a_{3}+t b_{3}\right) .
$$

Thus, even when $f$ is unknown, we can compute its specialization (6.6) directly from $I_{C}$.

When $\mathcal{S}$ is developable, the specialization (6.6) can also be obtained from the ideal $I\left(\mathcal{S}^{\vee}\right)$. Let $J$ be a Jacobian matrix for the ideal $I\left(\mathcal{S}^{\vee}\right)$ in $\mathbb{C}\left[y_{0}, y_{1}, y_{2}, y_{3}\right]$. This matrix has four columns. Let $J_{x}$ be the matrix obtained from $J$ by adding one more row, namely the vector $\left(x_{0}, x_{1}, x_{2}, x_{3}\right)$ in $(6.5)$. We now add the $3 \times 3-$ minors of $J_{x}$ to the ideal $I\left(\mathcal{S}^{\vee}\right)$, we saturate with respect to the ideal of $2 \times 2$ minors of $J$, and then we eliminate the unknowns $y_{0}, y_{1}, y_{2}, y_{3}$. The result is the desired principal ideal (6.6) in $\mathbb{C}[s, t]$. See Example 6.30 for an application.

These strategies can be adapted to compute the plane curve that is obtained as the intersection of a ruled or developable surface $\mathcal{S}$ with a fixed plane $H$ in $\mathbb{P}^{3}$. For event surfaces, this corresponds to restricting the camera movement to a plane, or to assuming that all projections are orthographic (which means that the viewpoint lies on the plane at infinity). It is sufficient to parametrize the points on $H$ by writing $x_{i}=s a_{i}+t b_{i}+u c_{i}$ in (6.6).

Associated ruled surfaces. The ruled surfaces of interest to us arise from an arbitrary curve or surface $X$ in $\mathbb{P}^{3}$. They represent families of planes and lines that intersect $X$ with prescribed multiplicities and are shown in the bottom rows of Figures 6.2 and 6.3 . For a general curve or surface $X$ in $\mathbb{P}^{3}$, the rows of these diagrams correspond to codimension in $\left(\mathbb{P}^{3}\right)^{*}$ or $\operatorname{Gr}\left(1, \mathbb{P}^{3}\right)$. The shown subvarieties consist of lines and planes that intersect $X$ with various multiplicities $m$. A solid edge from $Y_{1}$ to $Y_{2}$ means that $Y_{2}$ is an irreducible component of the singular locus of $Y_{1}$. A dashed edge just means that $Y_{2}$ is contained in $Y_{1}$. Below the ambient spaces $\left(\mathbb{P}^{3}\right)^{*}$ and $\operatorname{Gr}\left(1, \mathbb{P}^{3}\right)$ we see the coisotropic hypersurfaces associated to $X$. As we shall see in Sections 6.2 and 6.3, the irreducible components of the visual event surface of $X$ are (iterated) singular loci of these hypersurfaces. The developable components are dual to the singular curves in 
the dual surface $X^{\vee}$. The non-developable components are parametrized by the singular curves in the singular locus of the Chow or Hurwitz threefold of $X$.

We first consider a general smooth curve $X$ in $\mathbb{P}^{3}$. The left diagram in Figure 6.2 depicts the landscape in $\left(\mathbb{P}^{3}\right)^{*}$. The dual surface $X^{\vee}$ consists of planes that meet $X$ with multiplicity two. The singular locus of $X^{\vee}$ is the union of two irreducible curves, whose points are osculating planes $(m=3)$ and bitangent planes $(m=2+2)$. The symbols that denote our loci, like $\mathcal{T}^{p}(X)$ and $\mathcal{E}^{p}(X)$, will be explained in Sections 6.2 and 6.3. The right diagram in Figure 6.2 shows the landscape in the Grassmannian $\operatorname{Gr}\left(1, \mathbb{P}^{3}\right)$. We refer to Theorem 5.1 and Section 5.5 for precise statements and proofs, also for the right diagram in Figure 6.3. The singular locus of the Chow threefold $\mathrm{CH}_{0}(X)$ is the surface $\operatorname{Sec}(X)$ in $\operatorname{Gr}\left(1, \mathbb{P}^{3}\right)$ of secant lines, i.e., lines that meet $X$ twice. The singular locus of $\operatorname{Sec}(X)$ is the curve $\mathcal{D}^{\ell}(X)$ of trisecant lines. The curve $\mathcal{T}^{\ell}(X)$ of tangent lines is contained in $\operatorname{Sec}(X)$ but it does not belong to the singular locus.

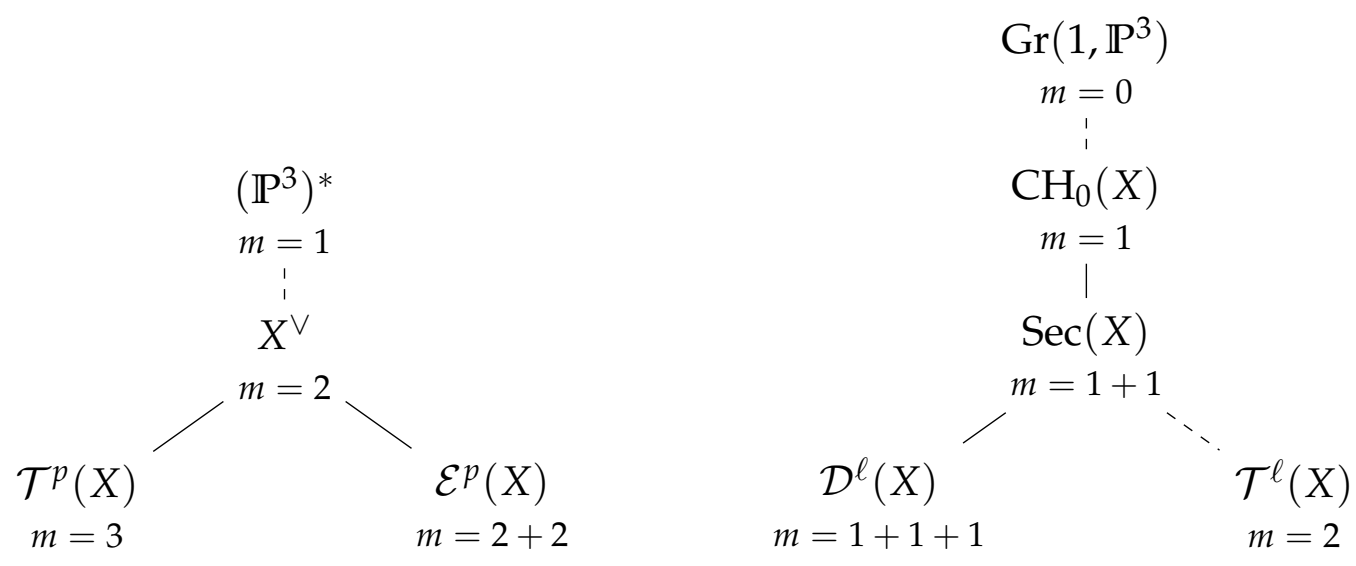

Figure 6.2: Loci of planes and lines that meet a curve $X$ with assigned multiplicities.

In Figure 6.3, we consider various loci associated with a general smooth surface $X$ in $\mathbb{P}^{3}$. The dual surface $X^{\vee}$ is singular along two irreducible curves. The nodal component $\mathcal{E}^{p}(X)$ of its singular locus is the set of all bitangent planes, and the cuspidal component $\mathcal{P}^{p}(X)$ is the set of all planes that intersect $X$ with multiplicity three at a point. The Hurwitz threefold $\mathrm{CH}_{1}(X)$ is singular along two irreducible surfaces. Its nodal component $\operatorname{Bit}(X)$ contains all bitangent lines, and its cuspidal component $\operatorname{Infl}(X)$ comprises all inflectional lines. These surfaces contain three special curves $\mathcal{F}^{\ell}(X), \mathcal{C}^{\ell}(X)$ and $\mathcal{T}^{\ell}(X)$, indicating lines that meet $X$ with multiplicity four, or $3+2$, or $2+2+2$. For instance, $2+2+2$ refers to tritangent lines. Sections 6.3, 6.4 and 6.5 are devoted to the ruled surfaces in $\mathbb{P}^{3}$ that are represented by these curves.

For each of the five curves at the bottom of Figure 6.3 , there is also an associated curve on $X$. It consists of the points on $X$ where the special intersection occurs. For example, the curve associated with $\mathcal{E}^{p}(X)$ is the locus of points on $X$ that lie on bitangent planes. These are the contact points on a curved object when it is rolled on a table. Our favorite terminology for this curve is due to 


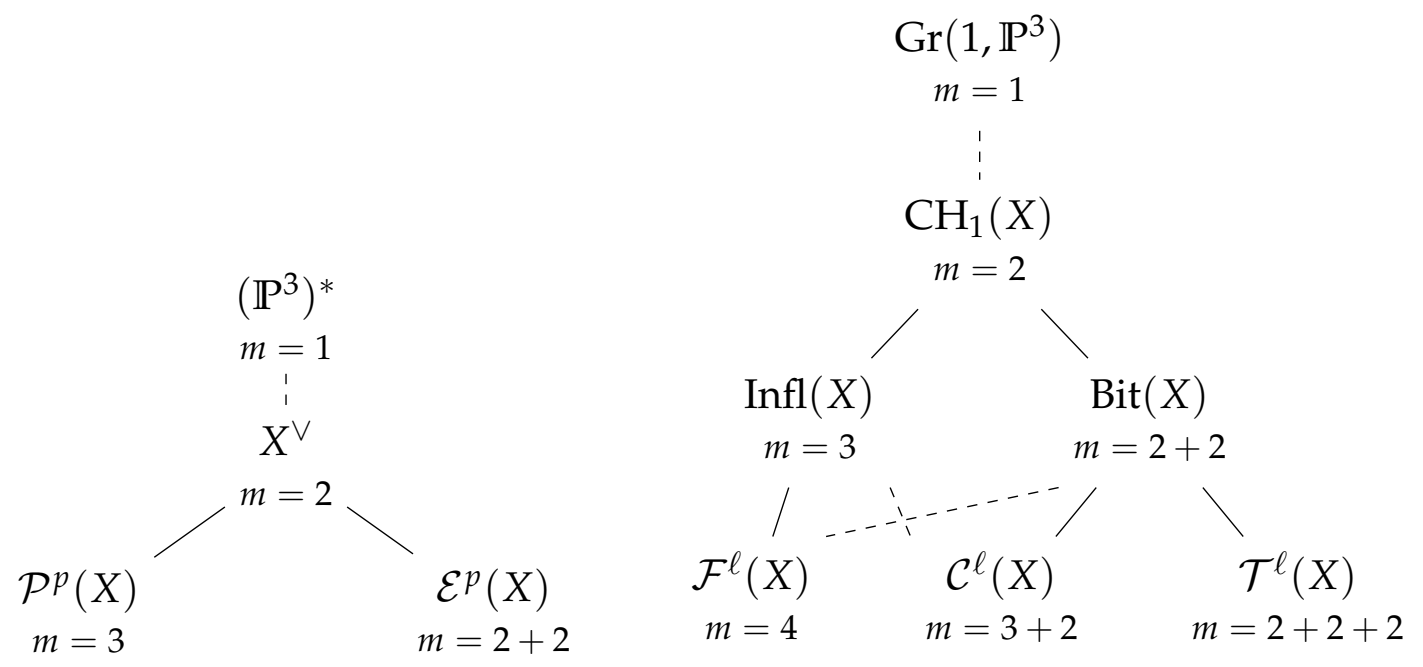

Figure 6.3: Loci of planes and lines that meet a surface $X$ with assigned multiplicities.

Cayley: he calls it the node-couple curve. For $\mathcal{P}^{p}(X)$ and $\mathcal{F}^{\ell}(X)$, the special contact occurs at a single point, and we can give a more detailed description. At a general point $x$, the surface has two inflectional lines (see Lemma 4.28). These are the tangent lines of the nodal curve obtained by intersecting $X=Z(f)$ with its tangent plane at $x$. The same lines are the intersection of the tangent plane with the Hessian quadric at $x$ defined by

$$
y \cdot H_{f}(x) \cdot y^{T}=0, \quad \text { where } y=\left(y_{0}: y_{1}: y_{2}: y_{3}\right) \text { and } H_{f}=\left(\frac{\partial^{2} f}{\partial x_{i} \partial x_{j}}\right)_{0 \leq i, j \leq 3} \text {. }
$$

Exceptional situations occur at flecnodal and parabolic points $x$. At a flecnodal point, one of the two inflectional lines has intersection multiplicity four. Such a line is called a flecnodal line. At a parabolic point, the Hessian matrix $H_{f}(x)$ drops rank, and the two inflectional lines degenerate to a double line. At these points, the intersection of $X$ with its tangent plane has a cusp at $x$. The locus of all parabolic points is the curve given by the intersection of $X$ with the Hessian surface $Z\left(\operatorname{det}\left(H_{f}\right)\right)$. Over the real numbers, the parabolic curve is the boundary between the elliptic and hyperbolic regions on $X$, where the two inflectional lines are respectively both complex or both real.

The curve $\mathcal{P}^{p}(X)$ is the set of tangent planes at parabolic points, and the curve $\mathcal{F}^{\ell}(X)=\mathcal{L}_{4}(X)$ is the set of flecnodal lines at flecnodal points. The parabolic and flecnodal curves always intersect tangentially, at special points known as godrons (or cusps of the Gauss map). Interestingly, the node-couple curve also passes through the godrons, and has the same tangent as the parabolic and flecnodal curves [17, p. 170]. 


\subsection{Views of Curves}

In this section, we study the visual events for a general curve $X$ in $\mathbb{P}^{3}$ of degree $d$ and genus $g$. In particular, $X$ is smooth and irreducible. The three visual events of $X$ correspond to the three Reidemeister moves that are familiar from knot theory. They are shown in Figure 6.4. For a general center $z$, the plane curve $C_{z}(X)$ has $\frac{1}{2}(d-1)(d-2)-g$ nodes and no other singularities (see Theorem 5.10). If $z$ is a point on the visual event surface of $X$, other singularities occur. The three components of the visual event surface are as follows:
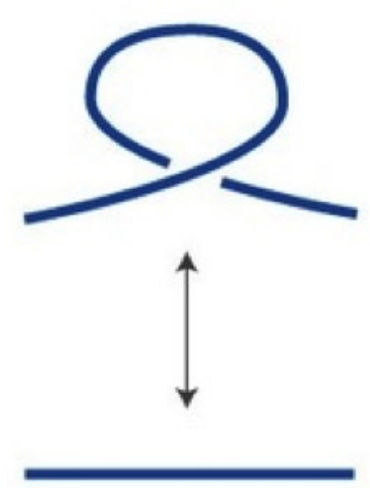
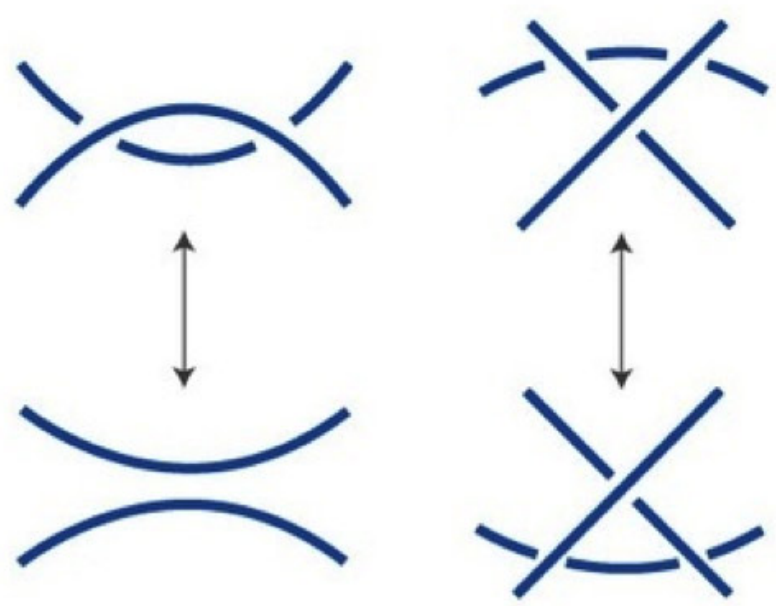

Figure 6.4: Changing views of a curve correspond to Reidemeister moves. The viewpoint $z$ crosses the tangential surface (left), edge surface (middle), or trisecant surface (right).

1. The tangential surface $\mathcal{T}(X)$, also known as the tangent developable, is the union of all tangent lines to $X$. It represents viewpoints $z$ such that the plane curve $C_{z}(X)$ has a cusp. When $z$ crosses $\mathcal{T}(X)$, a node on $C_{z}(X)$ transitions from being real to complex.

2. The edge surface $\mathcal{E}(X)$ is the union of all secant lines that are edges. An edge is the line spanned by two points on $X$ whose tangent lines lie in a common plane. This surface represents viewpoints $z$ such that the plane curve $C_{z}(X)$ has a tacnode. When $z$ crosses $\mathcal{E}(X)$, a pair of nodes transitions between being real and being complex.

3. The trisecant surface $\mathcal{D}(X)$ is the union of all lines that are spanned by triples of collinear points on $X$ (the symbol $\mathcal{D}$ stands for $d r e i)$. This represents viewpoints $z$ such that $C_{z}(X)$ has a triple point. When $z$ crosses $\mathcal{D}(X)$, the real curve $C_{z}(X)$ experiences a triangle crossing, but the real singularity structure is unchanged.

We use the coisotropic hypersurfaces of $X$ and their iterated singular loci to formally prove that the described surfaces are all components of the visual 
event surface of $X$. Consider the projection $\pi_{z}: X \subset \mathbb{P}^{3} \rightarrow-\rightarrow \mathbb{P}^{2}$ from a center $z \in \mathbb{P}^{3} \backslash X$. The target $\mathbb{P}^{2}$ has two intrinsic realizations. These live in the ambient spaces $\operatorname{Gr}\left(1, \mathbb{P}^{3}\right)$ and $\left(\mathbb{P}^{3}\right)^{*}$ respectively. The first is the surface $\alpha(z)$ of all lines in $\mathbb{P}^{3}$ that contain $z$. The second is the plane $z^{\vee}$ of all planes in $\mathbb{P}^{3}$ that contain $z$. Basic projective geometry yields the following characterizations of the image curve $C_{z}(X)$ in these intrinsic realizations of the image plane.

Proposition 6.4. The image $C_{z}(X)$ of our curve $X$ is projectively equivalent to the curve $\alpha(z) \cap \mathrm{CH}_{0}(X)$ in the Grassmannian $\operatorname{Gr}\left(1, \mathbb{P}^{3}\right)$. The dual curve $\left(C_{z}(X)\right)^{\vee}$ in $\left(\mathbb{P}^{2}\right)^{*}$ is projectively equivalent to the curve $z^{\vee} \cap X^{\vee}$ in the dual projective space $\left(\mathbb{P}^{3}\right)^{*}$.

In computer vision, the term visual cone is used for the union of all lines in the preimage of a set in $\mathbb{P}^{2}$. The visual cone of a curve is a developable surface in $\mathbb{P}^{3}$. The dual of the visual cone associated with $C_{z}(X)$ is the plane curve $z^{\vee} \cap X^{\vee}$. Hence, Proposition 6.4 describes the curves in $\operatorname{Gr}\left(1, \mathbb{P}^{3}\right)$ and $\left(\mathbb{P}^{3}\right)^{*}$ that encode this visual cone, as discussed after Theorem 6.2 .

Theorem 6.5. For a general space curve $X$ of degree $d \geq 3$ and genus $g$, the visual event surface $\mathcal{V}(X)$ is the branch locus of the finite projection

$$
\begin{aligned}
& \omega:\left\{(z, L) \in \mathbb{P}^{3} \times \operatorname{Gr}\left(1, \mathbb{P}^{3}\right) \mid z \in L, L \in \operatorname{Sing}\left(\mathrm{CH}_{0}(X)\right)\right\} \longrightarrow \mathbb{P}^{3} \text {, } \\
& (z, L) \longmapsto z \text {, }
\end{aligned}
$$

and has (at most) three irreducible components, namely $\mathcal{T}(X), \mathcal{E}(X)$, and $\mathcal{D}(X)$.

Proof. Let $z \in \mathbb{P}^{3} \backslash X$. Nodes in $C_{z}(X)$ correspond to points in $\operatorname{Sec}(X) \cap \alpha(z)$, i.e., to points in the fiber $\omega^{-1}(z)$ by Theorem 5.8. Hence, the curve $C_{z}(X)$ has the expected number $\frac{1}{2}(d-1)(d-2)-g$ of nodes if and only if $z$ is not in the branch locus of $\omega$. This is equivalent to that $\alpha(z)$ intersects $\operatorname{Sec}(X)$ only at smooth points and the intersection at all those points is transverse (cf. [34, Thm. 1.26]). Thus, by Corollary 5.36, there are exactly three cases when the number of nodes is different:

1. The congruence $\alpha(z)$ intersects the curve $\operatorname{Sing}(\operatorname{Sec}(X))$, which is the Zariski closure of the set of all trisecant lines of $X$.

2. The congruence $\alpha(z)$ intersects $\operatorname{Sec}(X)$ at some $L \in \operatorname{Reg}(\operatorname{Sec}(X))$ nontransversely:

a) either $L \cap X=\left\{x_{1}, x_{2}\right\}, x_{1} \neq x_{2}, L \neq \mathbb{T}_{X, x_{1}}$ and $L \neq \mathbb{T}_{X, x_{2}}$,

b) or $L \cap X=\{x\}$ and $L=\mathbb{T}_{X, x}$.

The first case yields directly the trisecant surface $\mathcal{D}(X)$. For the other two cases, we first observe that

$$
T_{\alpha(z), L}=\left\{\varphi \in \operatorname{Hom}\left(\boldsymbol{L}, \mathbb{A}^{4} / \boldsymbol{L}\right) \mid \boldsymbol{z} \subset \operatorname{ker}(\varphi)\right\},
$$

which we have derived in the proof of Proposition 4.14 . 
If we have a line $L$ as in Case $2 \mathrm{a}$, then $\alpha(z)$ and $\operatorname{Sec}(X)$ intersect non-transversely at $L$ if and only if the intersection of (5.4) and (6.7) contains a nonzero homomorphism. Since $z \notin X$, such a homomorphism exists if and only if $\left(\mathbb{T}_{X_{,} x_{1}}+\boldsymbol{L}\right) / \boldsymbol{L}=\left(\mathbb{T}_{X_{,} \boldsymbol{x}_{2}}+\boldsymbol{L}\right) / \boldsymbol{L}$, i.e., $\mathbb{T}_{\boldsymbol{X}, \boldsymbol{x}_{\mathbf{1}}}+\boldsymbol{L}=\mathbb{T}_{\boldsymbol{X}, \boldsymbol{x}_{\mathbf{2}}}+\boldsymbol{L}$, which means that $L$ is an edge. Thus, Case 2a yields the edge surface $\mathcal{E}(X)$.

If $L$ is a line as in Case 2b, then the intersection of (5.5) and (6.7) contains always a non-zero homomorphism, and $\alpha(z)$ and $\operatorname{Sec}(X)$ intersect non-transversely at $L$. Hence, Case $2 b$ yields the tangential surface $\mathcal{T}(X)$.

We use the symbols $\mathcal{T}^{\ell}(X), \mathcal{E}^{\ell}(X)$ and $\mathcal{D}^{\ell}(X)$ to denote the curves in the Grassmannian $\operatorname{Gr}\left(1, \mathbb{P}^{3}\right)$ that represent the surfaces $\mathcal{T}(X), \mathcal{E}(X)$ and $\mathcal{D}(X)$. Two of the curves appear on the lower right in Figure 6.2

The trisecant surface $\mathcal{D}(X)$ is ruled but not developable, so it has no associated curve in $\left(\mathbb{P}^{3}\right)^{*}$. The surfaces $\mathcal{T}(X)$ and $\mathcal{E}(X)$ are developable, so they can also be represented by their dual curves in $\left(\mathbb{P}^{3}\right)^{*}$. We use the same notation as the left diagram in Figure 6.2 .

$$
\mathcal{T}^{p}(X)=\mathcal{T}(X)^{\vee} \quad \text { and } \quad \mathcal{E}^{p}(X)=\mathcal{E}(X)^{\vee} .
$$

Here the index " $p$ " stands for planes. The earlier used upper index " $\ell$ " stands for lines. We have already explained in Section 6.1 that the curve $\mathcal{T}^{p}(X)$ of osculating planes to $X$ is dual to $\mathcal{T}(X)$ (see Figure 6.1] and [82, page 111]). The duality of the edge surface and the curve $\mathcal{E}^{p}(X)$ of bitangent planes is easy to see. Indeed, a general bitangent plane $H$ is tangent to $X$ at two distinct points $x, y \in H$. Since $\mathbb{T}_{X, x} \subset H$ and $\mathbb{T}_{X, y} \subset H$, the line $L$ spanned by $x$ and $y$ is an edge. Due to biduality, $x^{\vee}$ and $y^{\vee}$ are tangent planes to the curve $\mathcal{E}^{p}(X)$ at the point $H^{\vee}$. Hence, every point on $L$ corresponds to a tangent plane of this curve at $H^{\vee}$.

The following classical theorem characterizes the expected degrees of the ruled surfaces $\mathcal{T}(X), \mathcal{E}(X)$ and $\mathcal{D}(X)$.

Theorem 6.6. For a general space curve $X$ of degree $d$ and genus $g$, the degrees of the tangential surface $\mathcal{T}(X)$, the edge surface $\mathcal{E}(X)$ and the trisecant surface $\mathcal{D}(X)$ are as follows:

$$
\begin{array}{rcc}
\operatorname{deg}(\mathcal{T}(X)) & = & 2(d+g-1) \\
\operatorname{deg}(\mathcal{E}(X)) & = & 2(d-3)(d+g-1) \\
\operatorname{deg}(\mathcal{D}(X)) & = & \frac{(d-1)(d-2)(d-3)}{3}-(d-2) g .
\end{array}
$$

Proof. The degree of the tangential surface $\mathcal{T}(X)$ is the Riemann-Hurwitz number $2 d+2 g-2$. This coincides with the degree of the dual surface $X^{\vee}$. See $[82$. page 111] for a geometric derivation and [56] for computational examples. The formula for the degree of the edge surface $\mathcal{E}(X)$ appears in [88, Thm. 2.1]. The proof given there is based on De Jonquiéres' Formula. The degree of the trisecant surface $\mathcal{D}(X)$ is due to Berzolari who first found it in 1895. One finds Berzolari's formula in Bertin's article [8] on the geometry of $\mathcal{D}(X)$. 


\begin{tabular}{ccccc}
\hline$d$ & $g$ & $\operatorname{deg}(\mathcal{T}(X))$ & $\operatorname{deg}(\mathcal{E}(X))$ & $\operatorname{deg}(\mathcal{D}(X))$ \\
\hline 3 & 0 & 4 & 0 & 0 \\
4 & 0 & 6 & 6 & 2 \\
4 & 1 & 8 & 8 & 0 \\
5 & 0 & 8 & 16 & 8 \\
5 & 1 & 10 & 20 & 5 \\
5 & 2 & 12 & 24 & 2 \\
6 & 0 & 10 & 30 & 20 \\
6 & 1 & 12 & 36 & 16 \\
6 & 2 & 14 & 42 & 12 \\
6 & 3 & 16 & 48 & 8 \\
6 & 4 & 18 & 54 & 4 \\
\hline
\end{tabular}

Table 6.1: Degrees of the components of the visual event surface of a space curve.

Table 6.1 summarizes the conclusion of Theorem 6.6 for space curves of degree $d$ at most six. The genus $g$ ranges from 0 to Castelnuovo's bound. Note that, for fixed $d$ and increasing $g$, the degree of $\mathcal{D}(X)$ decreases while that of the others increases. In particular, there is no trisecant surface for twisted cubic curves and elliptic quartic curves (cf. [8, Prop. 1]).

The edge surface $\mathcal{E}(X)$ is of importance in convex geometry because the algebraic boundary of the convex hull of a real affine curve $X_{\mathbb{R}}$ consists of $\mathcal{E}(X)$ and the tritangent planes of $X$. This was shown by Ranestad and Sturmfels in [88, $\S 2]$, and in [88, §3] they describe a method for computing $\mathcal{E}(X)$ when $X$ is rational. This theme was picked up by Seigal and Sturmfels in their study of real tensor decompositions [97]. According to [97, §3], the real rank two boundary of $X$ is the union $\mathcal{T}(X) \cup \mathcal{E}(X)$, so it is part of the visual event surface of $X$. The study of curves in the present section is thus a further development of [88, 97].

Our task in this section is to solve the following computer algebra problem. Given a general space curve $X$, compute the ruled surfaces $\mathcal{T}(X), \mathcal{E}(X)$ and $\mathcal{D}(X)$. Here the input is either the ideal of $X$, or a parametric representation of $X$. The output is the defining polynomial $f$ of the surface $\mathcal{S}_{C}$ in $\mathbb{P}^{3}$. If the polynomial $f$ is too large, we compute the ideal of the curve $C$ in $\operatorname{Gr}\left(1, \mathbb{P}^{3}\right)$ or, when $\mathcal{S}_{C}$ is developable, the ideal of the dual $\mathcal{S}_{C}^{\vee}$ in $\left(\mathbb{P}^{3}\right)^{*}$.

Many space curves arising in applied contexts lie in the zero set of a quadratic polynomial. A generic curve has this property when its genus $g$ is maximal with respect to the Castelnuovo bound [50, Thm. 6.4, page 351]. We now focus on that special case. Later in this section we address our computational task for general curves that are not on a quadric.

Let $X$ be a general curve on a smooth quadric $Q$ in $\mathbb{P}^{3}$. Any line $L$ in $\mathbb{P}^{3}$ that intersects the quadric $Q$ in three points must lie on $Q$, so $L$ lies in one of the two rulings of $Q$.

Remark 6.7. If $d \geq 4$ and $(d, g) \neq(4,1)$ and $X$ lies on a quadric $Q$, then the trisecant surface $\mathcal{D}(X)$ coincides with the quadric surface $Q$, taken with an ap- 
propriate multiplicity.

To derive that multiplicity, and to set the stage for computing $\mathcal{T}(X)$ and $\mathcal{E}(X)$, we perform a linear change of coordinates in $\mathbb{P}^{3}$ so that the equation of $Q$ equals $x_{0} x_{3}=x_{1} x_{2}$. Thus, we identify $Q$ with the Segre surface $\mathbb{P}^{1} \times \mathbb{P}^{1}$. We fix affine coordinates $((1: s),(1: t))$.

Corollary 6.8. If $X$ has bidegree $(a, b)$ on $Q=\mathbb{P}^{1} \times \mathbb{P}^{1} \subset \mathbb{P}^{3}$, then the degrees of the tangential surface $\mathcal{T}(X)$, the edge surface $\mathcal{E}(X)$ and the (non-reduced) trisecant surface $\mathcal{D}(X)$ are

$$
\begin{array}{rlc}
\operatorname{deg}(\mathcal{T}(X)) & = & 2 a b, \\
\operatorname{deg}(\mathcal{E}(X)) & = & 2 a b(a+b-3), \\
\operatorname{deg}(\mathcal{D}(X)) & = & 2\left(\left(\begin{array}{c}
a \\
3
\end{array}\right)+\left(\begin{array}{c}
b \\
3
\end{array}\right)\right) .
\end{array}
$$

Proof. The affine polynomial $f(s, t)$ that defines $X$ has degree $a$ in $s$ and degree $b$ in $t$. Our curve $X$ has degree $d=a+b$ and genus $g=(a-1)(b-1)$. Indeed, it is a basic fact from toric geometry that the genus $g$ is the number of interior lattice points of the Newton polygon, which is a rectangle of size $a \times b$. Moreover, a general curve $X$ in $\mathbb{P}^{3}$ of that degree and genus lies on a quadric, so we can apply Theorem 6.6. We substitute $d=a+b$ and $g=(a-1)(b-1)$ into the formulas given there. This yields the formulas in Corollary 6.8 .

We now see that the "appropriate multiplicity" in Remark 6.7 is $\left(\begin{array}{l}a \\ 3\end{array}\right)+\left(\begin{array}{l}b \\ 3\end{array}\right)$. The two summands correspond to the two rulings of $Q$. Each line in the first ruling meets $X$ in $a$ points, so it counts as a trisecant with multiplicity $\left(\begin{array}{l}a \\ 3\end{array}\right)$, and ditto with $b$ for the second ruling.

We shall present algorithms for computing $\mathcal{T}(X)$ and $\mathcal{E}(X)$ from the affine polynomial $f(s, t)$ that defines $X$ in $\mathbb{P}^{1} \times \mathbb{P}^{1}$. Recall that a change of coordinates is required in order to apply our method in situations when $X$ is given by its ideal in $\mathbb{R}\left[x_{0}, x_{1}, x_{2}, x_{3}\right]$. We illustrate this point, and later our algorithms, for the case when $a=3, b=2$ and hence $d=5, g=2$.

Example 6.9. Let $X$ be the bicanonical embedding of a curve with genus two. It has degree five in $\mathbb{P}^{3}$. The curve is arithmetically Cohen-Macaulay. Its ideal is given by the $2 \times 2$-minors of

$$
\left[\begin{array}{lll}
\ell_{11} & \ell_{12} & q_{1} \\
\ell_{21} & \ell_{22} & q_{2}
\end{array}\right]
$$

The $\ell_{i j}$ are linear forms and the $q_{i}$ are quadratic forms, found by computing syzygies of $X$. Assuming $\ell_{11}, \ell_{12}, \ell_{21}, \ell_{22}$ to be linearly independent, we change coordinates and write this as

$$
\left[\begin{array}{ccc}
x_{0} & x_{2} & q_{1}\left(x_{0}, x_{1}, x_{2}, x_{3}\right) \\
x_{1} & x_{3} & q_{2}\left(x_{0}, x_{2}\right)
\end{array}\right]=\left[\begin{array}{ccc}
1 & t & g_{1}(s, t) \\
s & s t & g_{2}(t)
\end{array}\right] .
$$

Here, the polynomial $g_{1}$ has bidegree $(2,2)$ in $(s, t)$, the polynomial $g_{2}$ has degree two in $t$, and we used column operations to eliminate $x_{1}$ and $x_{3}$ from $q_{2}$. 
One of the $2 \times 2$-minors of this $2 \times 3$-matrix is the equation of bidegree $(3,2)$ that defines our curve in the affine plane:

$$
f(s, t)=g_{2}(t)-s g_{1}(s, t) .
$$

Conversely, every polynomial of bidegree $(3,2)$ in $(s, t)$ has such a matrix representation (6.8).

To compute the tangential surface $\mathcal{T}(X)$ from $f(s, t)$, we form the $3 \times 4$-matrix

$$
M=\left[\begin{array}{cccc}
1 & s & t & s t \\
0 & -f_{t} & f_{s} & s f_{s}-t f_{t} \\
x_{0} & x_{1} & x_{2} & x_{3}
\end{array}\right], \quad \text { where } f_{s}=\frac{\partial f}{\partial s} \text { and } f_{t}=\frac{\partial f}{\partial t}
$$

The first two rows of $M$ are linearly independent, and they span the tangent line at the point of $X$ corresponding to $(s, t)$. The second row is the image of the tangent direction $\left(-f_{t}, f_{s}\right)$ of the affine curve $\{f=0\}$ under the linear map given by the Jacobian of $\mathbb{C}^{2} \rightarrow \mathbb{C}^{3},(s, t) \mapsto(s, t, s t)$. Another point $\left(x_{0}: \ldots: x_{3}\right)$ lies on that tangent line in $\mathbb{P}^{3}$ precisely when $\operatorname{rank}(M)=2$. The following ideal is generated by five polynomials in $\mathbb{R}\left[s, t, x_{0}, x_{1}, x_{2}, x_{3}\right]$ :

$$
\langle f\rangle+\langle 3 \times 3 \text {-minors of } M\rangle \text {. }
$$

Our argument implies the following method for finding the tangential surface of degree $2 a b$ :

Proposition 6.10. Eliminating the unknowns s and t from the ideal (6.9) yields a principal ideal in $\mathbb{R}\left[x_{0}, x_{1}, x_{2}, x_{3}\right]$. Its generator is the polynomial defining the tangential surface $\mathcal{T}(X)$.

Example 6.11. Let $d=5, g=2$ as in Example 6.9 and fix the curve $X \subset \mathbb{P}^{1} \times \mathbb{P}^{1}$ defined by

$$
f=s^{3} t^{2}+s^{3}+t^{2}+s+t+1 .
$$

The polynomial that defines the tangential surface $\mathcal{T}(X)$ has degree 12 . It has 432 monomials, out of the $\left(\begin{array}{c}15 \\ 3\end{array}\right)=455$ possible ones of degree 12 , and it looks like this (see Code Fin the Appendix):

$$
\begin{array}{r}
93 x_{0}^{12}+310 x_{0}^{11} x_{1}+341 x_{0}^{10} x_{1}^{2}+558 x_{0}^{9} x_{1}^{3}+1054 x_{0}^{8} x_{1}^{4}+744 x_{0}^{7} x_{1}^{5}+837 x_{0}^{6} x_{1}^{6} \\
+\cdots+216 x_{1} x_{3}^{11}+108 x_{3}^{12} .
\end{array}
$$

We now consider the edge surface $\mathcal{E}(X)$. Its degree is $2 a b(a+b-3)$. We shall compute its dual representation $\mathcal{E}^{p}(X)$. Planes in $\mathbb{P}^{3}$ correspond to curves of bidegree $(1,1)$ in $\mathbb{P}^{1} \times \mathbb{P}^{1}$ :

$$
y_{0}+y_{1} s+y_{2} t+y_{3} s t=0
$$

We solve for $t$, substitute into $f(s, t)$, and clear denominators. The result is a polynomial in one variable $s$ of degree $a+b$. We seek the condition that this 
has two double roots, corresponding to $m=2+2$ in Figure 6.2. This condition defines the curve $\mathcal{E}^{p}(X)$ in $\left(\mathbb{P}^{3}\right)^{*}$.

Example 6.12. Let $d=5, g=2$ as before in Example 6.9. Fix the curve $X$ in Example 6.11. We shall compute the dual curve $\mathcal{E}^{p}(X)$ to the edge surface; see Code $\mathrm{G}$ in the Appendix for Macaulay2 code. The moving curve (6.11) has five intersection points with the fixed curve $f=0$ in $(6.10)$. Their s-coordinates are the roots of

$$
c_{0}+c_{1} s+c_{2} s^{2}+c_{3} s^{3}+c_{4} s^{4}+c_{5} s^{5}=0,
$$

where the $c_{i}$ are quadratic polynomials in $y_{0}, y_{1}, y_{2}, y_{3}$. Regarding the coefficients as unknowns, we pre-compute the ideal $\Delta_{(2,2)}(5) \subset \mathbb{R}\left[c_{0}, c_{1}, c_{2}, c_{3}, c_{4}, c_{5}\right]$ whose variety consists of quintics (6.12) with two double roots. The ideal $\Delta_{(2,2)}(5)$ has codimension 2 and degree 12 . It is generated by 10 quintics in the $c_{i}$, as seen in the row labeled $\lambda=221$ in [71, Table 1]. Let $I$ be the ideal obtained from $\Delta_{(2,2)}(5)$ by replacing the $c_{i}$ with the quadrics in $y_{0}, y_{1}, y_{2}, y_{3}$ that represent the specific curve $X$, and then saturating by the irrelevant ideal $\left\langle y_{0}, y_{1}, y_{2}, y_{3}\right\rangle$. The variety of $I$ is the curve $\mathcal{E}^{p}(X)$ in $\left(\mathbb{P}^{3}\right)^{*}$. The ideal $I$ has 14 minimal generators, all of degree 10, with large integer coefficients. This is the dual representation of the edge surface.

Computing $\mathcal{E}(X)$ by directly dualizing $\mathcal{E}^{p}(X)$ rarely terminates in practice. It is easier to intersect $\mathcal{E}(X)$ with lines or planes, as explained in Section 6.1. around (6.5) and (6.6).

We now consider curves $X$ that need not lie on a quadric $Q$. Let us first assume that $X$ is the image of a variety $Y$ in a higher-dimensional space $\mathbb{P}^{d}$ under a linear projection $\omega: \mathbb{P}^{d} \rightarrow-\mathbb{P}^{3}$. This allows us to preprocess $Y$, especially if the Chow form of $Y$ is known.

This approach works well when $X$ is rational. Here $Y$ is the rational normal curve in $\mathbb{P}^{d}$, parametrized by $\left(1: t: t^{2}: \cdots: t^{d}\right)$. Let $\omega: \mathbb{P}^{d} \rightarrow \mathbb{P}^{3}$ be the linear projection that maps $Y$ onto our curve $X$. We write $A$ for the $4 \times(d+1)$ matrix that represents $\omega$.

We first compute the tangential surface $\mathcal{T}(X)$. Let $s$ be an unknown and let $Q$ be the skew-symmetric $4 \times 4$-matrix obtained from (6.1) by substituting to dual Plücker coordinates. We form the ideal in $\mathbb{R}\left[s, q_{01}, q_{02}, \ldots, q_{23}\right]$ that is generated by the Plücker quadric $q_{01} q_{23}-q_{02} q_{13}+q_{03} q_{12}$ and the eight entries of the $4 \times 2$ matrix

$$
Q \cdot A \cdot\left[\begin{array}{ccccccc}
1 & s & s^{2} & s^{3} & s^{4} & \ldots & s^{d} \\
0 & 1 & 2 s & 3 s^{2} & 4 s^{3} & \cdots & d s^{d-1}
\end{array}\right]^{T} .
$$

Eliminating $s$ and saturating with respect to the $q_{i j}$ now yields the ideal of the curve $\mathcal{T}^{\ell}(X)$. From this we can compute the defining polynomial of $\mathcal{T}(X)$ via (6.1). The edge surface $\mathcal{E}(X)$ can be computed similarly. This was also discussed in [88, §2] and in [97, §3].

We now compute the trisecant surface $\mathcal{D}(X)$ of a rational curve $X$ of degree $d$ in $\mathbb{P}^{3}$. The Chow form of $Y$ is the resultant of two binary forms of degree $d$. We write this as the determinant of the Bézout matrix $B(r)$. This is a symmetric 
$d \times d$-matrix whose entries are linear forms in the Plücker coordinates $r_{i j}$ of $(d-2)$-planes in $\mathbb{P}^{d}$. For the formula we refer to equation (1.18) on page 402 in Section III.12.1 of [43]. The Bézout matrix for $d=6$ equals

$$
B(r)=\left[\begin{array}{cccccc}
r_{01} & r_{02} & r_{03} & r_{04} & r_{05} & r_{06} \\
r_{02} & r_{03}+r_{12} & r_{04}+r_{13} & r_{05}+r_{14} & r_{06}+r_{15} & r_{16} \\
r_{03} & r_{04}+r_{13} & r_{05}+r_{14}+r_{23} & r_{06}+r_{15}+r_{24} & r_{16}+r_{25} & r_{26} \\
r_{04} & r_{05}+r_{14} & r_{06}+r_{15}+r_{24} & r_{16}+r_{25}+r_{34} & r_{26}+r_{35} & r_{36} \\
r_{05} & r_{06}+r_{15} & r_{16}+r_{25} & r_{26}+r_{35} & r_{36}+r_{45} & r_{46} \\
r_{06} & r_{16} & r_{26} & r_{36} & r_{46} & r_{56}
\end{array}\right]
$$

We shall use the following fact that is well-known in computer algebra; see [1. page 1228].

Lemma 6.13. The minors of the Bézout matrix $B(r)$ having size $d-k+1$ define an irreducible variety of codimension $k$ in the Grassmannian of $(d-2)$-planes in $\mathbb{P}^{d}$. General points $q$ on this variety represent pairs of univariate polynomials of degree $d$ that have $k$ common zeros.

The second exterior power $\wedge_{2} A$ of the matrix $A$ is a matrix of format $6 \times\left(\begin{array}{c}d+1 \\ 2\end{array}\right)$. We write $p=\left(p_{01}, p_{02}, p_{03}, p_{12}, p_{13}, p_{23}\right)$ for the Plücker coordinates of a line in $\mathbb{P}^{3}$. The preimage of the line $p$ under the projection $\alpha$ is the $(d-2)$-plane in $\mathbb{P}^{d}$ with Plücker coordinates $r=p \cdot \wedge_{2} A$.

Proposition 6.14. If the $4 \times(d+1)$-matrix $A$ is sufficiently general, then the ideal of $(d-2) \times(d-2)$-minors of the matrix $B\left(p \cdot \wedge_{2} A\right)$ defines the curve $\mathcal{D}^{\ell}(X)$ of degree $2\left(\begin{array}{c}d-1 \\ 3\end{array}\right)$ in $\mathbb{P}^{5}$.

Proof. We use Lemma 6.13. Intersecting the curve $X$ with two planes in $\mathbb{P}^{3}$ amounts to solving two univariate polynomials of degree $d$. The Chow form of $X$ is the determinant of the specialized Bézout matrix $B\left(p \cdot \wedge_{2} A\right)$. The corank of that matrix is the number of common zeros. That number is three when the intersection of the two planes is a trisecant line of $X$. Thus, the line $p$ is in the trisecant curve $\mathcal{D}^{\ell}(X)$ precisely when $B\left(p \cdot \wedge_{2} A\right)$ has rank $\leq d-3$. By Lemma 6.1 and setting $g=0$ in Theorem 6.6, the degree of $\mathcal{D}^{\ell}(X)$ is $2\left(\begin{array}{c}d-1 \\ 3\end{array}\right)$.

For an illustration consider rational curves $X$ of degree $d=6$. Then $A$ is a $4 \times 7$ matrix, and $\wedge_{2} A$ is a $6 \times 21$ matrix. The ideal of $4 \times 4$-minors of the $6 \times 6$-matrix $B\left(p \cdot \wedge_{2} A\right)$ is minimally generated, modulo the Plücker relation, by 45 quartics. After saturating with respect to the irrelevant maximal ideal $\left\langle p_{01}, p_{02}, p_{03}, p_{12}, p_{13}, p_{23}\right\rangle$, we obtain the prime ideal $I_{C}$ of the curve $C$ of trisecants; see Code $\mathrm{H}$ in the Appendix. The ideal $I_{C}$ has degree 20 and is generated by 10 cubics.

The success of this computation relied on writing the Chow form of $X$ as a determinant of a matrix whose entries are linear in Plücker coordinates. Eisenbud, Schreyer and Weyman [35, §4] proved that such a formula exists for all curves. See [35, Prop. 4.2] for a derivation of the Bézout matrix $B(r)$ from the 
perspective of Ulrich sheaves, and [35, Example 4.6] for an extension to hyperelliptic curves. Whenever we have such matrices explicitly, we get the surface $\mathcal{D}(X)$ by imposing the corank 3 constraints. Such matrix formulas for Chow forms can also be derived for curves $X$ in $\mathbb{P}^{3}$ that arise by intersecting certain nice varieties.

Example 6.15. The Macaulay2 code for the following computations is listed in Code I in the Appendix. Let $X$ be the curve in $\mathbb{P}^{3}$ defined by the $3 \times 3$-minors of the $3 \times 4$-matrix

$$
M(x)=\left[\begin{array}{cccc}
x_{0}+x_{3} & x_{1}-x_{0} & x_{2}-x_{1} & x_{2}+x_{3} \\
x_{3}-x_{2} & x_{0}+x_{3} & x_{1}-x_{0} & x_{1}+x_{2} \\
x_{2}-x_{1} & x_{1}-x_{2}+x_{3} & x_{0}+x_{3} & x_{0}
\end{array}\right] .
$$

This curve has $(d, g)=(6,3)$. By computing syzygies, we can represent every curve of degree six and genus three via such a matrix with linear entries. This follows from the Hilbert-Burch Theorem. Let $Y$ be the variety of $3 \times 4$-matrices of rank $\leq 2$. The Chow form of $Y$ is the determinant of the following $6 \times 6$-matrix in dual Plücker coordinates for lines in $\mathbb{P}^{11}$ :

$$
\left[\begin{array}{cccccc}
r_{00,01} & r_{00,11}+r_{10,01} & r_{00,21}+r_{20,01} & r_{10,11} & r_{10,21}+r_{20,11} & r_{20,21} \\
r_{00,02} & r_{00,12}+r_{10,02} & r_{00,22}+r_{20,02} & r_{10,12} & r_{10,22}+r_{20,12} & r_{20,22} \\
r_{00,03} & r_{00,13}+r_{10,03} & r_{00,23}+r_{20,03} & r_{10,13} & r_{10,23}+r_{20,13} & r_{20,23} \\
r_{01,02} & r_{01,12}+r_{11,02} & r_{01,22}+r_{21,02} & r_{11,12} & r_{11,22}+r_{21,12} & r_{21,22} \\
r_{01,03} & r_{01,13}+r_{11,03} & r_{01,23}+r_{21,03} & r_{11,13} & r_{11,23}+r_{21,13} & r_{21,23} \\
r_{02,03} & r_{02,13}+r_{12,03} & r_{02,23}+r_{22,03} & r_{12,13} & r_{12,23}+r_{22,13} & r_{22,23}
\end{array}\right] .
$$

This matrix appears in [43, page 472]. We now replace the Plücker coordinates $r_{i j, k l}$ by linear forms in the six coordinates $q_{i j}=a_{i} b_{j}-b_{i} a_{j}$ for lines in $\mathbb{P}^{3}$. For instance, $r_{00,01}=q_{01}+q_{03}-q_{13}, r_{00,02}=-q_{01}+q_{02}+q_{13}-q_{23}, r_{00,03}=q_{02}+$ $q_{03}-q_{23}, \ldots$. These linear forms are obtained by setting $r_{i j, k l}=M(a)_{i j} M(b)_{k l}-$ $M(b)_{i j} M(a)_{k l}$ where $a=\left(a_{0}, a_{1}, a_{2}, a_{3}\right)$ and $b=\left(b_{0}, b_{1}, b_{2}, b_{3}\right)$.

The trisecant curve $\mathcal{D}^{\ell}(X)$ is defined in $\operatorname{Gr}\left(1, \mathbb{P}^{3}\right) \subset \mathbb{P}^{5}$ by the $4 \times 4$-minors of the resulting matrix (6.13). Saturating by $\left\langle q_{01}, q_{02}, q_{03}, q_{12}, q_{13}, q_{23}\right\rangle$ yields the prime ideal of $\mathcal{D}^{\ell}(X)$ :

$$
\begin{aligned}
I= & \left\langle q_{03} q_{12}-q_{02} q_{13}+q_{01} q_{23}, q_{02} q_{12}-q_{12}^{2}-q_{03} q_{13}-q_{13}^{2}+q_{01} q_{23}-2 q_{02} q_{23}+2 q_{03} q_{23}+2 q_{12} q_{23},\right. \\
& q_{01} q_{12}-q_{12}^{2}-q_{01} q_{13}+q_{02} q_{13}-3 q_{03} q_{13}-q_{12} q_{13}-q_{01} q_{23}+2 q_{03} q_{23}+3 q_{12} q_{23}+q_{13} q_{23}-2 q_{23}^{2}, \\
& q_{03}^{2}+q_{03} q_{13}+q_{13}^{2}-q_{01} q_{23}-2 q_{03} q_{23}-q_{12} q_{23}+q_{23}^{2}, q_{02}^{2}-q_{12}^{2}-q_{03} q_{13}-2 q_{02} q_{23}+q_{03} q_{23}+2 q_{12} q_{23}, \\
& q_{01} q_{03}-2 q_{03} q_{13}-q_{12} q_{13}-2 q_{13}^{2}+q_{01} q_{23}+2 q_{03} q_{23}+2 q_{12} q_{23}+2 q_{13} q_{23}-2 q_{23}^{2} \\
& \left.q_{01} q_{02}+2 q_{02} q_{03}-q_{12}^{2}-q_{01} q_{13}-q_{02} q_{13}-2 q_{03} q_{13}-q_{12} q_{13}+3 q_{12} q_{23}+2 q_{13} q_{23}-2 q_{23}^{2}\right\rangle .
\end{aligned}
$$

From this, we easily find the octic equation of the trisecant surface $\mathcal{D}(X)$ :

$$
x_{0}^{7} x_{2}-2 x_{0}^{4} x_{1}^{3} x_{2}+x_{0} x_{1}^{6} x_{2}+2 x_{0}^{5} x_{1} x_{2}^{2}+2 x_{0}^{4} x_{1}^{2} x_{2}^{2}+2 x_{0}^{3} x_{1}^{3} x_{2}^{2}-2 x_{0}^{2} x_{1}^{4} x_{2}^{2}+\cdots
$$

This polynomial uses 136 of the $165=\left(\begin{array}{c}8+3 \\ 3\end{array}\right)$ monomials of degree eight.

The past few pages were devoted to specialized techniques that exploit the 
structure of a given curve $X$. Such techniques can be designed for all entries in Table 6.1. However, equally important are general purpose methods that work for all curves. We close this section by discussing the latter. The curve $X$ is given by its ideal $I=\left\langle f_{1}, f_{2}, \ldots, f_{k}\right\rangle$ in $\mathbb{R}\left[x_{0}, x_{1}, x_{2}, x_{3}\right]$.

The edge surface $\mathcal{E}(X)$ was already discussed in [88, 97]. We therefore focus on the other two ruled surfaces in Theorem 6.6. The easier among them is the tangential surface $\mathcal{T}(X)$. At any given point $p$ on the curve $X$, the tangent line is defined by the linear equations

$$
\left[\begin{array}{cccc}
\frac{\partial f_{1}}{\partial x_{0}}(p) & \frac{\partial f_{1}}{\partial x_{1}}(p) & \frac{\partial f_{1}}{\partial x_{2}}(p) & \frac{\partial f_{1}}{\partial x_{3}}(p) \\
\vdots & \vdots & \vdots & \vdots \\
\frac{\partial f_{k}}{\partial x_{0}}(p) & \frac{\partial f_{k}}{\partial x_{1}}(p) & \frac{\partial f_{k}}{\partial x_{2}}(p) & \frac{\partial f_{k}}{\partial x_{3}}(p)
\end{array}\right] \cdot\left(\begin{array}{c}
x_{0} \\
x_{1} \\
x_{2} \\
x_{3}
\end{array}\right)=\left(\begin{array}{l}
0 \\
0 \\
0 \\
0
\end{array}\right) .
$$

To find the polynomial $F$ defining $\mathcal{T}(X)$, we take a vector of variables $p=$ $\left(y_{0}, y_{1}, y_{2}, y_{3}\right)$, and we augment $I$ with the constraints (6.14). This gives an ideal in $\mathbb{R}\left[x_{0}, x_{1}, x_{2}, x_{3}, y_{0}, y_{1}, y_{2}, y_{3}\right]$. From that ideal, we saturate and eliminate the variables $y_{0}, y_{1}, y_{2}, y_{3}$. The output is $\langle F\rangle$.

The trisecant surface $\mathcal{D}(X)$ will be represented by its curve $\mathcal{D}^{\ell}(X)$ in the Grassmannian $\operatorname{Gr}\left(1, \mathbb{P}^{3}\right)$. To compute this, we parametrize the line in $\mathbb{P}^{3}$ as in (6.2). Suppose for now that our curve is a complete intersection: $X=Z\left(f_{1}, f_{2}\right)$. We want the univariate polynomials $f_{1}(z(t))$ and $f_{2}(z(t))$ to have three common roots, i.e., their greatest common divisor (GCD) has degree at least three. This can be expressed using subresultants [1]. The vanishing of all subresultants of order $i=0, \ldots, r-1$ for two polynomials in $t$ means that their GCD has degree at least $r$. In our case, we form the ideal given by the subresultant coefficients of $f_{1}(z(t))$ and $f_{2}(z(t))$ of order 0,1 and 2 (together with the Plücker relation). The ideal of the trisecant curve $\mathcal{D}^{\ell}(X)$ is obtained by saturating by the ideal of the leading coefficients of $f_{1}(z(t))$ and $f_{2}(z(t))$.

This approach generalizes to the case when $X$ is not a complete intersection. Indeed, if $X$ is defined by $f_{1}, \ldots, f_{k}$, then we can use the same strategy to impose that $s_{1} f_{1}(z(t))+\cdots+s_{k-1} f_{k-1}(z(t))$ and $f_{k}(z(t))$ have three roots in common for any choice of $s_{1}, \ldots, s_{k-1}$.

We conclude this section with an example that illustrates the last row of Table 6.1.

Example 6.16. Let $X$ be the smooth curve of degree six and genus four in $\mathbb{P}^{3}$ defined by

$$
x_{0}^{2}+x_{1}^{2}+x_{2}^{2}+x_{3}^{2}=x_{0}^{3}+x_{1}^{3}+x_{2}^{3}+x_{3}^{3}=0 .
$$

The above method easily yields the equation of degree 18 for the tangential surface $\mathcal{T}(X)$ :

$$
\begin{array}{r}
4 x_{0}^{12} x_{1}^{6}-12 x_{0}^{12} x_{1}^{5} x_{2}-12 x_{0}^{12} x_{1}^{5} x_{3}+21 x_{0}^{12} x_{1}^{4} x_{2}^{2}+\cdots+\underline{13770} x_{0}^{6} x_{1}^{4} x_{2}^{4} x_{3}^{4} \\
+\cdots+24 x_{2}^{7} x_{3}^{11}+4 x_{2}^{6} x_{3}^{12} .
\end{array}
$$

This polynomial has 1094 terms. Its largest coefficient is underlined. It is also 
easy to compute the quartic surface $\mathcal{D}(X)$ with the above method. This is indeed a quadric with multiplicity two, defined by

$$
x_{0}^{2}+x_{1}^{2}+x_{2}^{2}+x_{3}^{2}=0 .
$$

The Macaulay2 code for these computations is given in Code『lin the Appendix.

\subsection{Views of Surfaces}

We now turn to the visual events for a general surface $X$ in $\mathbb{P}^{3}$. For a general center $z$, the contour $C_{z}(X)$ has $\frac{1}{2} d(d-1)(d-2)(d-3)$ nodes, $d(d-1)(d-2)$ cusps and no other singularities (see Theorem 5.18). Special situations occur at the six visual events associated with $X$, which were mentioned at the very beginning of this chapter in items (L) and (M). We shall explain these events and how they give rise to the following five irreducible surfaces:

1. The flecnodal surface $\mathcal{F}(X)$ is the union of all lines $L$ with contact of order four at a point of $X$. In other words, the equation of $X$ restricted to $L$ has a root of multiplicity four.

2. The cusp crossing surface $\mathcal{C}(X)$ is the union of all lines $L$ with contact of order $3+2$ at two points of $X$, i.e., the equation for $X \cap L$ on $L$ has a triple root and a double root.

3. The tritangent surface $\mathcal{T}(X)$ is the union of all lines $L$ with contact of order $2+2+2$ at three points of $X$, i.e., the equation for $X \cap L$ on $L$ has three double roots.

4. The edge surface $\mathcal{E}(X)$ is the envelope of the bitangent planes of $X$. It is the union of all bitangent lines contained bitangent planes with the same two points of tangency. This surface was denoted $\left(X^{[2]}\right)^{\vee}$ in [88].

5. The parabolic surface $\mathcal{P}(X)$ is the envelope of all tangent planes that have contact of order three with $X$. It is the union of all inflectional lines at parabolic points [78, §A.1.2].

Consider again the projection $\pi_{z}: X \subset \mathbb{P}^{3} \rightarrow-\rightarrow \mathbb{P}^{2}$ from a center $z \in \mathbb{P}^{3} \backslash X$. The following result, analogous to Proposition 6.4, describes intrinsic realizations of the contour $C_{z}(X)$.

Proposition 6.17. The contour $C_{z}(X)$ of our surface $X$ is projectively equivalent to the curve $\alpha(z) \cap \mathrm{CH}_{1}(X)$ in the Grassmannian $\operatorname{Gr}\left(1, \mathbb{P}^{3}\right)$. The curve $\left(C_{z}(X)\right)^{\vee}$ in $\left(\mathbb{P}^{2}\right)^{*}$ that is dual to the contour is projectively equivalent to the curve $z^{\vee} \cap X^{\vee}$ in the dual projective space $\left(\mathbb{P}^{3}\right)^{*}$. 
We use again the coisotropic hypersurfaces of $X$ and their iterated singular loci to give a formal proof on the irreducible components of the visual event surface of $X$.

Theorem 6.18. For a general surface $X$ of degree at least four, the visual event surface $\mathcal{V}(X)$ is the branch locus of the finite projection

$$
\begin{aligned}
& \omega:\left\{(z, L) \in \mathbb{P}^{3} \times \operatorname{Gr}\left(1, \mathbb{P}^{3}\right) \mid z \in L, L \in \operatorname{Sing}\left(\mathrm{CH}_{1}(X)\right)\right\} \longrightarrow \mathbb{P}^{3}, \\
& (z, L) \longmapsto z \text {, }
\end{aligned}
$$

and has (at most) five irreducible components: $\mathcal{F}(X), \mathcal{C}(X), \mathcal{T}(X), \mathcal{E}(X), \mathcal{P}(X)$.

Proof. Let $z \in \mathbb{P}^{3} \backslash X$. Nodes in $C_{z}(X)$ correspond to points $\operatorname{in} \operatorname{Bit}(X) \cap \alpha(z)$ and cusps in $C_{z}(X)$ to points in $\operatorname{Infl}(X) \cap \alpha(z)$. Note that the union of these points corresponds to points in the fiber $\omega^{-1}(z)$ by Theorem 5.13. Hence, there are the expected numbers $\frac{1}{2} d(d-1)(d-2)(d-3)$ of nodes and $d(d-1)(d-2)$ of cusps in $C_{z}(X)$ if and only if $z$ is not in the branch locus of $\omega$. This is equivalent to that $\alpha(z)$ intersects both $\operatorname{Bit}(X)$ and $\operatorname{Infl}(X)$ only at smooth points and the intersection at all those points is transverse (cf. [34, Thm. 1.26]). According to Theorem 5.37, Proposition 5.39 and [5, Lem. 4.3] (see also the discussion before Conjecture 5.40), there are exactly the following three cases when the number of nodes or cusps is different:

1. The congruence $\alpha(z)$ intersects $\mathcal{T}^{\ell}(X), \mathcal{C}^{\ell}(X)$ or $\mathcal{F}^{\ell}(X)$ (see Figure 6.3).

2. The congruence $\alpha(z)$ intersects $\operatorname{Bit}(X)$ at some $L \in \operatorname{Reg}(\operatorname{Bit}(X))$ nontransversely.

3. The congruence $\alpha(z)$ intersects $\operatorname{Infl}(X)$ at some $L \in \operatorname{Reg}(\operatorname{Infl}(X))$ nontransversely.

The first case yields the tritangent, cusp crossing and flecnodal surfaces.

Let us consider the second case. We can assume that $L$ is not a flecnodal line, since those lines are already covered by Case 1 . Thus, $L$ is tangent to $X$ at exactly two points $x_{1}, x_{2}$, and $L$ has contact order of exactly two at both $x_{1}$ and $x_{2}$. The congruence $\alpha(z)$ intersects $\operatorname{Bit}(X)$ non-transversely at $L$ if and only if the intersection of (5.6) and (6.7) contains a non-zero homomorphism. Since $z \notin X$, such a homomorphism exists if and only if $\mathbb{T}_{\boldsymbol{X}, \boldsymbol{x}_{\mathbf{1}}} / L=\mathbb{T}_{\boldsymbol{X}, \boldsymbol{x}_{\mathbf{2}}} / L$, i.e., $\mathbb{T}_{X, x_{1}}=\mathbb{T}_{X, x_{2}}$, which means that $L$ is a bitangent line contained in a bitangent plane. Hence, this case yields the edge surface $\mathcal{E}(X)$.

Finally, we focus on the third case. The Segre variety in Lemma 5.45 is a quadric surface in $\mathbb{P}^{3}$. Hence, every line is either contained in it, or intersects it at exactly two distinct points, or intersects it at just one point with multiplicity two. If $L \in \operatorname{Reg}(\operatorname{Infl}(X))$ is inflectional at a non-parabolic point $x$ of $X$, then, by Lemma 5.45, the projectivization of $T_{\operatorname{Infl}(X), L}$ intersects the Segre variety at exactly one point $\varphi$, whose kernel is $\boldsymbol{x}$. Since (6.7) contains only homomorphisms of rank one and $z \notin X$, the intersection of (6.7) and $T_{\operatorname{Infl}(X), L}$ is $\{0\}$. Thus, $\alpha(z)$ and $\operatorname{Infl}(X)$ intersect transversely at inflectional lines at non-parabolic points. If 
$L \in \operatorname{Reg}(\operatorname{Infl}(X))$ is inflectional at a parabolic point, then $\alpha(z)$ and $\operatorname{Infl}(X)$ intersect non-transversely at $L$, since (6.7) and (5.7) clearly contain a common nonzero homomorphism. All in all, Case 3 yields the parabolic surface $\mathcal{P}(X)$.

The following theorem characterizes the expected degree of the irreducible components of $\mathcal{V}(X)$.

Theorem 6.19. For a general surface $X$ of degree $d$ in $\mathbb{P}^{3}$, the degrees of the five ruled components of $\mathcal{V}(X)$ listed above are:

$$
\begin{aligned}
& \operatorname{deg}(\mathcal{F}(X))=\quad 2 d(d-3)(3 d-2), \quad \text { [94, §597] and [77, Prop. 4.5] } \\
& \operatorname{deg}(\mathcal{C}(X))=d(d-3)(d-4)\left(d^{2}+6 d-4\right), \quad \text { [94, §598] and [77, Prop. 4.12] } \\
& \operatorname{deg}(\mathcal{T}(X))=\frac{1}{3} d(d-3)(d-4)(d-5)\left(d^{2}+3 d-2\right) \text {, [94, §599] and [77, Prop. 4.10] } \\
& \operatorname{deg}(\mathcal{E}(X))=d(d-2)(d-3)\left(d^{2}+2 d-4\right), \quad \text { [94, §613] and [77, Prop. 4.16] } \\
& \operatorname{deg}(\mathcal{P}(X))=2 d(d-2)(3 d-4) . \quad \text { [94, §608] and [77, Prop. 4.3] }
\end{aligned}
$$

We first learned these degree formulas from Petitjean's article [77]. We then discovered that all five formulas already appeared in Salmon's 1882 book [94]. The precise pointers to both sources are given above. In Section 6.4. we present new proofs that are self-contained, except for pointers to the textbook [34].

The five ruled surfaces in Theorem 6.19 are encoded by the curves shown in the last row in Figure 6.3. The surfaces $\mathcal{E}(X)$ and $\mathcal{P}(X)$ are developable, and are the duals of the singular loci shown on the left in Figure 6.3 (see next paragraph). The remaining three surfaces $\mathcal{T}(X), \mathcal{C}(X)$ and $\mathcal{F}(X)$ arise from the curves in the Grassmannian $\operatorname{Gr}\left(1, \mathbb{P}^{3}\right)$ seen on the right of that diagram.

The duality of the edge surface with the curve of bitangent planes is analogous to the proof in the case of a curve $X$. For the duality of the parabolic surface of a surface $X$, we denote by $P \subset X$ the parabolic curve on $X$. Every plane $H \in\left(\mathcal{P}^{p}(X)\right)^{\perp}$ is tangent to $X$ at a parabolic point $p \in P$. We will see in Lemma 6.28 that the inflectional line $L$ at a general parabolic point $p \in P$ is spanned by $p$ and the unique projective point in the kernel of the Hessian matrix $H_{f}(p)$, where $f$ is a defining polynomial of $X$. We consider the Gauss map $\gamma: P \rightarrow \mathcal{P}^{p}(X)$ restricted to $P$. Taking the differential of $\gamma$ at $p$, we get

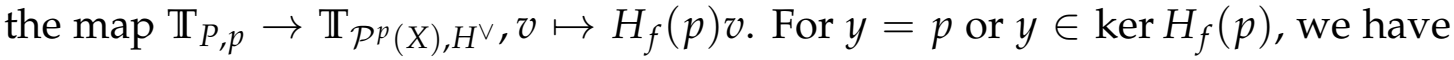
$y^{T} H_{f}(p) v=0$. This means that every point in $L$ corresponds to a tangent plane of $\mathcal{P}^{p}(X)$ at $H^{\vee}$.

The curves of lines and planes from Figure 6.3 capture both the local and multi-local features of the surface $X$. This is an advantage compared to the traditional approach for studying the appearance of surfaces based on differential geometry and singularity theory. In the computer vision literature [10, 78, 84, 85, 90], prominent local features of a surface were defined in terms of the euclidean Gauss map and the asymptotic spherical image. These are maps from the surface to the unit sphere $S^{2}$, taking a point on $X_{\mathbb{R}}$ to its normal direction, or to the direction of one of its inflectional lines. In our algebro-geometric setting, the role of $S^{2}$ is played by the dual surface $X^{\vee} \subset\left(\mathbb{P}^{3}\right)^{*}$ and the inflectional congruence $\operatorname{Infl}(X) \subset \operatorname{Gr}\left(1, \mathbb{P}^{3}\right)$. These surfaces carry much more information than the unit sphere $S^{2}$. 
We now describe the special singularities of the curve $C_{z}(X)$ that arise by projecting $X$ from $z \in \mathcal{V}(X)$. Given a point $u \in C_{z}(X)$, we write $L_{u}=\pi_{z}^{-1}(u) \in$ $\operatorname{Gr}\left(1, \mathbb{P}^{3}\right)$ for its fiber under $\pi_{z}$. Additionally to singularities of the contour, it is also interesting to learn (e.g. from [84]) that if $L_{u}$ is a (non-inflectional) tangent line at a parabolic point, then $u$ is a flex point of $C_{z}(X)$. Here, we are interested in higher order singularities seen in the image curve for special viewpoints:

$(\mathcal{T})$ If $L_{u}$ is a tritangent line, then $u$ is a triple point. This is a triple point event.

$(\mathcal{C})$ If $L_{u}$ is an inflectional bitangent, then $C_{z}(X)$ has a smooth branch and a cuspidal branch that meet at $u$. This is a cusp crossing event.

$(\mathcal{F})$ If $L_{u}$ is a flecnodal line, then $u$ is the limit of two cusps and a node, i.e., an infinitesimal change of the viewpoint produces two cusps and a node. This is a swallowtail event.

$(\mathcal{E})$ If $L_{u}$ is a bitangent line on a bitangent plane, then $u$ is a tacnode. It is obtained as the limit of two smooth branches coming together at $u$. This is a tangent crossing event.

$(\mathcal{P})$ If $L_{u}$ is the inflectional tangent at a parabolic point $p$, then, over the real numbers, two behaviors are possible: either $u$ is an isolated node, which corresponds to a lip event, or $u$ is a tacnode, obtained as the limit of two cusps, which is a beak-to-beak event.

The triple point, cusp crossing, and tangent crossing events are multi-local. The six visual events are shown in Figure 6.5. Detailed renderings of these pictures are ubiquitous in the relevant computer vision literature. For instance, see [78, Figures 5 and 6], and Figures 13.20 through 13.25 in the textbook [38].

We now briefly explain how to distinguish the two possible local behaviors (lip versus beak-to-beak) of the contour when the viewpoint $z$ belongs to the parabolic surface $\mathcal{P}(X)$. As argued before, the parabolic surface $\mathcal{P}(X)$ is a developable surface, since it is dual to the curve $\mathcal{P}^{p}(X)$ in $\left(\mathbb{P}^{3}\right)^{*}$. In particular, all inflectional lines at parabolic points are the tangents of the edge of regression curve, denoted by $E(\mathcal{P}(X))$. This allows us to associate each parabolic point $x$ with another point $e_{x}$, where the inflectional line at $x$ is tangent to $E(\mathcal{P}(X))$. In real projective three-dimensional space, the complement of $\left\{x, e_{x}\right\}$ in that line has two connected components. The distinction between lip and beak-to-beak is made by which of these two components the viewpoint $z$ belongs to. This was shown in [83, Thm. 4.10].

We next offer an informal discussion that should provide an intuitive understanding of our five event surfaces. The following are some real life situations where these events can actually be observed. We encourage our readers to look at the world from multiple viewpoints, and to then spot the six pictures of Figure 6.5. Do look carefully at the objects that surround you.

We first note that cuspidal and nodal singularities of image contours are stable features, which are visible in most surfaces. Nodes occur whenever occlusions create discontinuities in the contour. Cusps only appear for non-convex 

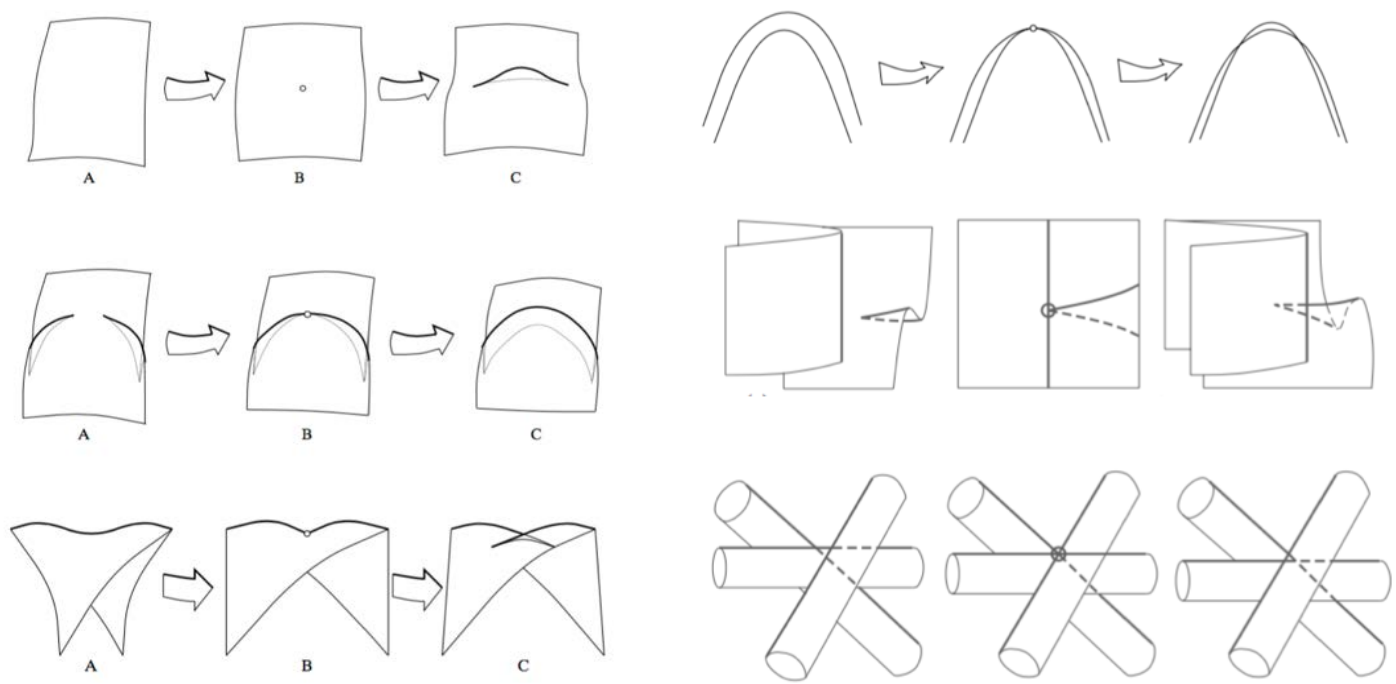

Figure 6.5: The catalogue of visual events for the projections of a smooth surface from a viewpoint that moves. The local events (left, from top to bottom) are lip, beak-to-beak, swallowtail. The multi-local events (right, from top to bottom) are tangent crossing, cusp crossing, triple point. Reprinted from [76] with permission of Springer.

objects. For instance, they can be observed on the folds of a piece of cloth. From an exceptional viewpoint, it is possible that several of these singularities occur along the same visual ray. This gives rise to a multi-local visual event (cusp crossing, tangent crossing, or triple points). Try it with a napkin or towel.

The three local events on the left in Figure 6.5 are more complicated. Videos of these events and their corresponding ruled surfaces are available at

https://github.com/kathlenkohn/thesis-material

in the folder chapter6. The readme file in that folder gives further explanations to the videos. The Sage code that was used to create the visualizations depicted in the videos can be found in the file Examples.ipynb.

It takes some practice to discover the local events in the real world. Here are some concrete examples that we found helpful:

- Lip event: If we observe a small hill from a high aerial viewpoint (say, from a hot air balloon), then all points on the ground are visible. The hill does not generate an image contour. However, as we descend closer to the ground, the profile of the hill suddenly becomes visible in the contour. This qualitative change of appearance is a lip event.

- Beak-to-beak. Observe a glass bottle from the bottom, with your eye close to the base. You see a part of the contour generated by the convex region where the sectional diameter of the bottle decreases. Now, tilt the bottle slowly towards its upright position. At some point, you see a complete path from the base to the top of the bottle. Previously your view had 
been blocked. This is a beak-to-beak event. Contrary to the lip event, the contour does not disappear at the transition point, but it breaks into two pieces.

- Swallowtail. The traditional drawing of a (transparent) torus presents two swallowtails. We see both cuspidal and nodal singularities in the contour [90, Fig. 2]. As we rotate the torus, a visual event occurs, and these singularities disappear. Try it with a bagel.

\subsection{Intersection Theory}

In this section, we derive the degrees of $\mathcal{E}^{p}(X), \mathcal{P}^{p}(X), \mathcal{P}(X)$ and $\mathcal{F}(X)$, and we sketch the relevant ideas for $\mathcal{E}(X), \mathcal{C}(X)$ and $\mathcal{T}(X)$. We found Petitjean's proofs in [77] to be lengthy and hard. They require a full understanding of Colley's multiple point theory [25, 26], and several of the steps are left out. By contrast, the derivations in Salmon's book [94] are inspiring but they lack the rigor of 20th century intersection theory.

The exposition that follows refers to the textbook by Eisenbud and Harris [34]. We believe that students of that book will find this section to be useful as supplementary reading.

We have discussed that the surfaces $\mathcal{E}(X)$ and $\mathcal{P}(X)$ are represented by their dual curves in $\left(\mathbb{P}^{3}\right)^{*}$. These are the irreducible components in the singular locus of the dual surface $X^{\vee}$.

Proposition 6.20. The degrees of the curves dual to the edge and parabolic surface of a general surface $X$ of degree $d \geq 4$ or $d \geq 3$, respectively, are

$$
\begin{aligned}
\operatorname{deg}\left(\mathcal{E}^{p}(X)\right) & =\frac{1}{2} d(d-1)(d-2)\left(d^{3}-d^{2}+d-12\right) \quad \text { and } \\
\operatorname{deg}\left(\mathcal{P}^{p}(X)\right) & =4 d(d-1)(d-2) .
\end{aligned}
$$

Proof. We count the bitangent and parabolic planes that contain a general point $z \in \mathbb{P}^{3}$. Consider the branch curve $C_{z}(X) \subset \mathbb{P}^{2}$. A bitangent plane to $X$ containing $z$ maps onto a bitangent line of $C_{z}(X)$. A tangent plane to $X$ at a parabolic point that contains $z$ maps onto a flex line of $C_{z}(X)$. These correspond, respectively, to the nodes and the cusps of the dual curve $C_{z}(X)^{\vee}$ in $\left(\mathbb{P}^{2}\right)^{*}$. We denote the number of nodes of $C_{z}(X)^{\vee}$ by $v_{2}=\operatorname{deg}\left(\mathcal{E}^{p}(X)\right)$ and the number of cusps of $C_{z}(X)^{\vee}$ by $\kappa_{2}=\operatorname{deg}\left(\mathcal{P}^{p}(X)\right)$.

We collect several results that can be found in the proof of Theorem 5.18 . the plane curve $C_{z}(X)$ has degree $d(d-1)$. Moreover, the degree of the dual curve $\left(C_{z}(X)\right)^{\vee} \subset\left(\mathbb{P}^{2}\right)^{*}$ is $d(d-1)^{2}$. The number of nodes in $C_{z}(X)$, which is the number of bitangent lines to $X$ passing through the general point $z$, is $v_{1}:=\frac{1}{2} d(d-1)(d-2)(d-3)$. The number of cusps in $C_{z}(X)$ is the number of inflectional lines to $X$ passing through $z$, which is $\kappa_{1}:=d(d-1)(d-2)$. 
We now apply Plücker's formula (see Lemma 5.15) to the dual plane curve $C_{z}(X)^{\vee}$ to derive

$$
\begin{aligned}
d(d-1)=\operatorname{deg}\left(C_{z}(X)\right) & =\operatorname{deg}\left(\left(C_{z}(X)\right)^{\vee}\right)\left(\operatorname{deg}\left(\left(C_{z}(X)\right)^{\vee}\right)-1\right)-2 \nu_{2}-3 \kappa_{2} \\
& =d(d-1)^{2}\left(d(d-1)^{2}-1\right)-2 \nu_{2}-3 \kappa_{2} .
\end{aligned}
$$

The dual curves $C_{z}(X)$ and $\left(C_{z}(X)\right)^{\vee}$ have the same geometric genus. By the degree-genus formula, it is

$$
\frac{1}{2}(d(d-1)-1)(d(d-1)-2)-v_{1}-\kappa_{1}=\frac{1}{2}\left(d(d-1)^{2}-1\right)\left(d(d-1)^{2}-2\right)-v_{2}-\kappa_{2} .
$$

Solving the equations (6.15) and (6.16) for $v_{2}$ and $\kappa_{2}$ leads to the formulas in Proposition 6.20.

This proof was entirely classical. By contrast, we derive the degrees of the visual event surfaces using modern intersection theory [34]. At the heart of intersection theory lies the Chow ring (see Section 5.4). In the following, we denote the rational equivalence class of a point in an irreducible projective variety $Y$ by $* \in A^{\operatorname{dim} Y}(Y)$, whenever this is well-defined. Moreover, we use the following two generators of the Chow ring of the Grassmannian $\operatorname{Gr}\left(1, \mathbb{P}^{3}\right)$ : the class $\gamma_{1}$ of the threefold of all lines that meet a given line (denoted by $\sigma_{1}$ in Section 5.4), and the class $\gamma_{2}$ of the surface of all lines that lie in a given plane (denoted by $\sigma_{1,1}$ in Section (5.4). The products (5.2) show that

$$
\begin{aligned}
& A^{0}\left(\operatorname{Gr}\left(1, \mathbb{P}^{3}\right)\right) \cong \mathbb{Z} \cdot\left[\operatorname{Gr}\left(1, \mathbb{P}^{3}\right)\right], \quad A^{1}\left(\operatorname{Gr}\left(1, \mathbb{P}^{3}\right)\right) \cong \mathbb{Z} \gamma_{1}, \quad A^{2}\left(\operatorname{Gr}\left(1, \mathbb{P}^{3}\right)\right) \cong \mathbb{Z} \gamma_{1}^{2} \oplus \mathbb{Z} \gamma_{2}, \\
& A^{3}\left(\operatorname{Gr}\left(1, \mathbb{P}^{3}\right)\right) \cong \mathbb{Z} \gamma_{1} \gamma_{2}, \quad A^{4}\left(\operatorname{Gr}\left(1, \mathbb{P}^{3}\right)\right) \cong \mathbb{Z} *, \quad \text { and } \\
& \gamma_{1}^{3}=2 \gamma_{1} \gamma_{2}, \quad \gamma_{2}^{2}=*, \quad \gamma_{1}^{2} \gamma_{2}=*, \quad \gamma_{1}^{4}=2 * .
\end{aligned}
$$

Further, the class $\gamma_{1}^{2}-\gamma_{2}$ represents the surface of all lines passing through a given point, which was denoted by $\sigma_{2}$ in Section 5.4 .

Example 6.21 ([34, Prop. 9.10]). The universal line

$$
\Phi:=\left\{(x, L) \in \mathbb{P}^{3} \times \operatorname{Gr}\left(1, \mathbb{P}^{3}\right) \mid x \in L\right\}
$$

is a five-dimensional smooth projective variety. The Chow ring of this variety equals $A^{*}(\Phi)=A^{*}\left(\operatorname{Gr}\left(1, \mathbb{P}^{3}\right)\right)[H] /\left\langle H^{2}-\gamma_{1} H+\gamma_{2}\right\rangle$. Here, the class $H \in A^{1}(\Phi)$ represents the preimage of a plane in $\mathbb{P}^{3}$ under the projection $\Phi \rightarrow \mathbb{P}^{3}$ onto the first factor.

We give an overview of the most crucial varieties, morphisms and classes, which are used throughout the following proofs, in Figure 6.6. 


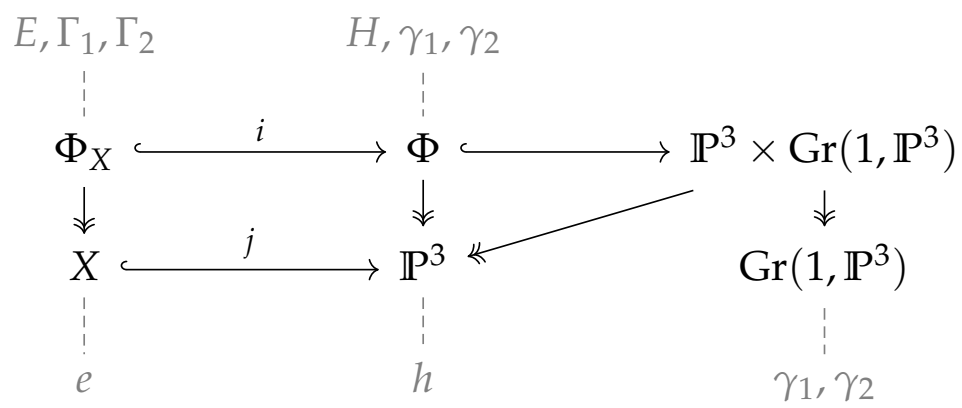

Figure 6.6: Commutative diagram of some morphisms appearing in this section. Classes in the Chow rings of the depicted varieties are represented in gray.

Proposition 6.22. For a general surface $X$ of degree $d \geq 4$, the degrees of the flecnodal surface $\mathcal{F}(X)$ and the flecnodal curve $F$ on $X$ are

$$
\operatorname{deg}(\mathcal{F}(X))=2 d(d-3)(3 d-2) \quad \text { and } \operatorname{deg}(F)=d(11 d-24) .
$$

Proof. Let $X_{\Phi}^{4} \subset \Phi$ be the incidence variety of pairs $(x, L)$ such that the line $L$ has contact of order at least four at the point $x \in X$. The degree of the flecnodal surface $\mathcal{F}(X)$ is the number of intersections of $\mathcal{F}(X)$ with a general line. This is the number of pairs $(x, L) \in X_{\Phi}^{4}$ such that $L$ meets a general line. In particular, we have $\left[X_{\Phi}^{4}\right] \cdot \gamma_{1}=\operatorname{deg}(\mathcal{F}(X)) *$ in $A^{*}(\Phi)$.

We shall compute $\left[X_{\Phi}^{4}\right] \in A^{4}(\Phi)$ via Chern classes. Fix any vector bundle $\mathcal{E}$ on $\Phi$ and any integer $m \in \mathbb{Z}_{>0}$. By [34, Thm. 11.2], there is a new vector bundle $\mathcal{J}_{\Phi / \mathrm{Gr}}^{m}(\mathcal{E})$ on $\Phi$ whose fiber at $(x, L)$ is the space of all germs of sections of $\left.\mathcal{E}\right|_{\{(y, L) \in \Phi\}}$ modulo those that vanish to order $\geq m+1$ at $(x, L)$. This is called the bundle of relative principal parts or the relative jet bundle. We shall compute its top Chern class.

Let now $\mathcal{E}$ be the pullback to $\Phi$ of the line bundle $\mathcal{O}_{\mathbb{P}^{3}}(d)$. A global section of $\mathcal{E}$ is given by the homogeneous polynomial in $x_{0}, x_{1}, x_{2}, x_{3}$ of degree $d$ that defines $X$ in $\mathbb{P}^{3}$. Restricting this polynomial to the line $L$ gives a global section of $\left.\mathcal{E}\right|_{\{(y, L) \in \Phi\}}$. Thus, we get a global section of $\mathcal{J}_{\Phi / \mathrm{Gr}}^{m}(\mathcal{E})$. That global section vanishes at $(x, L)$ if and only if $L$ has contact of order at least $m+1$ at $x \in X$. Hence, the top Chern class of $\mathcal{J}_{\Phi / \mathrm{Gr}}^{m}(\mathcal{E})$ is the class of the subvariety of all pairs $(x, L)$ in $\Phi$ such that $L$ has contact of order at least $m+1$ at $x \in X$. In particular, $c_{4}\left(\mathcal{J}_{\Phi / \mathrm{Gr}}^{3}(\mathcal{E})\right)=\left[X_{\Phi}^{4}\right]$.

In addition, we see from [34, Thm. 11.2] that $\mathcal{J}_{\Phi / \mathrm{Gr}}^{m}(\mathcal{E})$ agrees locally with

$$
\mathcal{E} \oplus\left(\mathcal{E} \otimes \Omega_{\Phi / G r}\right) \oplus\left(\mathcal{E} \otimes \operatorname{Sym}^{2} \Omega_{\Phi / G r}\right) \oplus \cdots \oplus\left(\mathcal{E} \otimes \operatorname{Sym}^{m} \Omega_{\Phi / G r}\right),
$$

where $\Omega_{\Phi / G r}$ is the relative cotangent bundle, which has rank one in our case. We compute the top Chern class of $\mathcal{J}_{\Phi / \mathrm{Gr}}^{m}(\mathcal{E})$ from its representation (6.17). From [34, p. 395] we have $c_{1}\left(\operatorname{Sym}^{m} \Omega_{\Phi / G r}\right)=m\left(\gamma_{1}-2 H\right)$ in $A^{1}(\Phi)$. Since 
the equation $f$ of $X$ gives a global section of $\mathcal{E}$, we further have $c_{1}(\mathcal{E})=d H$ in $A^{1}(\Phi)$. The top Chern class of the tensor product of two line bundles is the sum of their top Chern classes [34, Prop. 5.17]. Hence

$c_{1}\left(\mathcal{E} \otimes \operatorname{Sym}^{i} \Omega_{\Phi / G r}\right)=(d-2 i) H+i \gamma_{1} \quad$ in $\quad A^{1}(\Phi)$ for $i=0,1,2, \ldots, m$.

Finally, the Whitney Sum Formula [34, Thm. 5.3] asserts that the top Chern class of a direct sum of vector bundles is the product of the top Chern classes of the summands. This implies

$$
c_{m+1}\left(\mathcal{J}_{\Phi / \mathrm{Gr}}^{m}(\mathcal{E})\right)=\prod_{i=0}^{m}\left((d-2 i) H+i \gamma_{1}\right) \quad \text { in } \quad A^{m+1}(\Phi) .
$$

Example 5.25 implies $H^{4}=0$. Since exactly one line meets two general lines and passes through a given point, we have $H^{3} \gamma_{1}^{2}=*$. Finally, $H^{2} \gamma_{1}^{3}=2 *$ and $H \gamma_{1}^{4}=2 *$, since exactly two lines meet four general lines in $\mathbb{P}^{3}$. Putting these pieces together, we get the desired formula

$$
\begin{aligned}
\operatorname{deg}(\mathcal{F}(X)) * & =\left[X_{\Phi}^{4}\right] \cdot \gamma_{1}=c_{4}\left(\mathcal{J}_{\Phi / \mathrm{Gr}}^{3}(\mathcal{E})\right) \cdot \gamma_{1}=\gamma_{1} \prod_{i=0}^{3}\left((d-2 i) H+i \gamma_{1}\right) \\
& =\left(6 d^{3}-44 d^{2}+72 d\right) H^{3} \gamma_{1}^{2}+\left(11 d^{2}-36 d\right) H^{2} \gamma_{1}^{3}+6 d H \gamma_{1}^{4} \\
& =2 d(d-3)(3 d-2) * .
\end{aligned}
$$

An analogous computation yields the degree of the flecnodal curve $F$ on $X$ : $\operatorname{deg}(F) *=\left[X_{\Phi}^{4}\right] \cdot H=H \prod_{i=0}^{3}\left((d-2 i) H+i \gamma_{1}\right)=d(11 d-24) *$.

Proposition 6.23. The degree of the parabolic surface of a general surface $X$ of degree $d \geq 3$ is

$$
\operatorname{deg}(\mathcal{P}(X))=2 d(d-2)(3 d-4)
$$

Proof. We consider the incidence variety $P_{\Phi}^{3}$ that consists of all pairs $(x, L)$ in $\Phi$ with the property that $x$ is parabolic on $X$ and $L$ has contact order at least three at $x \in X$. Set-theoretically, this is the intersection of the variety $X_{\Phi}^{3} \subset \Phi$ of pairs $(x, L)$ such that $L$ is an inflectional line to $X$ at $x$ with the variety $P_{\Phi}^{1} \subset \Phi$ of pairs $(x, L)$ such that $x$ is parabolic on $X$. Since $\operatorname{codim}_{\Phi} P_{\Phi}^{3}=4<3+2=$ $\operatorname{codim}_{\Phi} X_{\Phi}^{3}+\operatorname{codim}_{\Phi} P_{\Phi}^{1}$, we cannot simply multiply the classes of $X_{\Phi}^{3}$ and $P_{\Phi}^{1}$ in $A^{*}(\Phi)$ to get the class of $P_{\Phi}^{3}$.

Instead, we shall compute all relevant classes in $A^{*}\left(\Phi_{X}\right)$, where $\Phi_{X}$ is the variety of pairs $(x, L) \in \Phi$ with $x \in X$. The varieties $X_{\Phi}^{3}$ and $P_{\Phi}^{1}$ intersect with the expected codimension in $\Phi_{X}$, although this intersection is not transverse. It is difficult to describe the Chow ring of $\Phi_{X}$. We find some generators and relations of $A^{*}\left(\Phi_{X}\right)$ by taking pullbacks of elements in $A^{*}(\Phi)$ under the inclusion $\Phi_{X} \hookrightarrow \Phi$. Recall that the pullback of a class $[Z]$ under a nice enough morphism $f$ between varieties is $\left[f^{-1}(Z)\right]$, by [34, Thm. 1.23]. We denote by $E \in A^{1}\left(\Phi_{X}\right)$ 
the pullback of the hyperplane class $H \in A^{1}(\Phi)$ and by $\Gamma_{i} \in A^{*}\left(\Phi_{X}\right)$ the pullback of $\gamma_{i} \in A^{*}(\Phi)$ for $i \in\{1,2\}$ (see Figure 6.6).

By definition, $P_{\Phi}^{1}$ is the preimage of the parabolic curve $P \subset X$ under the map $\Phi_{X} \rightarrow X$. The parabolic curve $P$ is the intersection of $X$ with the Hessian surface of degree $4(d-2)$. Thus, the class of $P$ in $A^{*}(X)$ is $4(d-2) e$, where $e \in A^{1}(X)$ is the pullback of the hyperplane class $h \in A^{1}\left(\mathbb{P}^{3}\right)$ under the inclusion $X \hookrightarrow \mathbb{P}^{3}$. As $E \in A^{1}\left(\Phi_{X}\right)$ is also the pullback of $e \in A^{1}(X)$ under the projection $\Phi_{X} \rightarrow X$, we have $\left[P_{\Phi}^{1}\right]=4(d-2) E \in A^{1}\left(\Phi_{X}\right)$.

We compute $\left[X_{\Phi}^{3}\right]$ in the Chow ring of $\Phi$ using the formula 6.17 with $m=2$ :

$$
\begin{aligned}
{\left[X_{\Phi}^{3}\right] } & =c_{3}\left(\mathcal{J}_{\Phi / \mathrm{Gr}}^{2}(\mathcal{E})\right)=\prod_{i=0}^{2}\left((d-2 i) H+i \gamma_{1}\right) \\
& =d H\left(\left(d^{2}-6 d+8\right) H^{2}+(3 d-8) H \gamma_{1}+2 \gamma_{1}^{2}\right) .
\end{aligned}
$$

The right hand side lives in the Chow ring of the five-dimensional variety $\Phi$ and we pull it back to the Chow ring of its four-dimensional subvariety $\Phi_{X}$. That pullback is the cycle $d E \beta$ with $\beta:=\left(d^{2}-6 d+8\right) E^{2}+(3 d-8) E \Gamma_{1}+2 \Gamma_{1}^{2}$ in $A^{2}\left(\Phi_{X}\right)$. Since pullback preserves codimension, $\left[X_{\Phi}^{3}\right] \in A^{2}\left(\Phi_{X}\right)$ cannot be equal to $d E \beta \in A^{3}\left(\Phi_{X}\right)$. Instead [34, Thm. 13.7] tells us that $d E \beta=\left[X_{\Phi}^{3}\right]$. $c_{1}\left(\mathcal{N}_{\Phi_{X} / \Phi}\right) \in A^{3}\left(\Phi_{X}\right)$, where $\mathcal{N}_{\Phi_{X} / \Phi}$ is the normal bundle of $\Phi_{X}$ in $\Phi$. By [34, Prop.-Def. 6.15], we have $c_{1}\left(\mathcal{N}_{\Phi_{X} / \Phi}\right)=d E$ and $d E \beta=d E\left[X_{\Phi}^{3}\right]$.

We cannot yet say that $\left[X_{\Phi}^{3}\right]=\beta$ in $A^{2}\left(\Phi_{X}\right)$ because multiplication with $E$ has a non-trivial kernel. However, since $E$ is a factor of $\left[P_{\Phi}^{1}\right]$, we conclude that $\left[X_{\Phi}^{3}\right]\left[P_{\Phi}^{1}\right]=4(d-2) E \beta$ in the Chow ring of $\Phi_{X}$. Since a general point on $X$ has two inflectional lines, the intersection multiplicity of the varieties $P_{\Phi}^{1}$ and $X_{\Phi}^{3}$ is two. Therefore, by [34, Thm. 1.26], we have $\left[P_{\Phi}^{3}\right]=\frac{1}{2}\left[X_{\Phi}^{3}\right]\left[P_{\Phi}^{1}\right]=2(d-2) E \beta$ in $A^{*}\left(\Phi_{X}\right)$. As in the case of flecnodal lines, the degree of the parabolic surface $\mathcal{P}(X)$ is the number of points in the zero-dimensional cycle

$$
\left[P_{\Phi}^{3}\right] \cdot \Gamma_{1}=2(d-2)\left(\left(d^{2}-6 d+8\right) E^{3} \Gamma_{1}+(3 d-8) E^{2} \Gamma_{1}^{2}+2 E \Gamma_{1}^{3}\right)
$$

Finally, we use the pushforward of the inclusion $i: \Phi_{X} \hookrightarrow \Phi$ to express the above monomials in the point class $*$ of $A^{*}(\Phi)$. The pushforward $f_{*}$ of a proper morphism $f$ maps $[Z]$ to 0 if $\operatorname{dim}(f(Z))<\operatorname{dim}(Z)$, and otherwise to $v[f(Z)]$ where $v \in \mathbb{Z}$ denotes the degree of the restricted map $\left.f\right|_{Z}: Z \rightarrow f(Z)$. Using the push-pull formula [34, Thm. 1.23], we derive

$$
i_{*}\left(E^{2} \Gamma_{1}^{2}\right)=i_{*}\left(i^{*}\left(H^{2} \gamma_{1}^{2}\right) \cdot\left[\Phi_{X}\right]\right)=H^{2} \gamma_{1}^{2} \cdot i_{*}\left(\left[\Phi_{X}\right]\right)=d H^{3} \gamma_{1}^{2}=d *
$$

Similarly, we get $i_{*}\left(E \Gamma_{1}^{3}\right)=2 d *$ and $i_{*}\left(E^{3} \Gamma_{1}\right)=0$. Hence, we conclude that $\operatorname{deg}(\mathcal{P}(X)) *=i_{*}\left(\left[P_{\Phi}^{3}\right] \cdot \Gamma_{1}\right)=2 d(d-2)(3 d-4) *$.

Proposition 6.24. The degree of the edge surface of a general surface X of degree $d \geq 4$ is

$$
\operatorname{deg}(\mathcal{E}(X))=d(d-2)(d-3)\left(d^{2}+2 d-4\right) \text {. }
$$


Proof sketch. We describe an idea for computing the degree of $\mathcal{E}(X)$ with a mix of classical methods and intersection theory, similar to Petitjean's approach [77, Prop. 4.14-4.16]. Since the degree of $\mathcal{E}(X)=\left(\mathcal{E}^{p}(X)\right)^{\vee}$ is the degree of the tangential surface of the curve $\mathcal{E}^{p}(X)$, we can use a generalization of the first formula in Theorem 6.6 to singular curves. We have already computed the degree of $\mathcal{E}^{p}(X)$ in Proposition 6.20, so we have to compute the genus of this curve. We will achieve this by first computing the degree and genus of the node-couple curve.

The Gauss map $\gamma: X \rightarrow\left(\mathbb{P}^{3}\right)^{*}$ assigns to each point $x \in X$ the tangent plane to $X$ at $x$. The preimage of $\mathcal{E}^{p}(X)$ under $\gamma$ is the node-couple curve $C \subset X$, and the restriction $\left.\gamma\right|_{C}: C \rightarrow \mathcal{E}^{p}(X)$ is a 2-to-1 covering of $\mathcal{E}^{p}(X)$. From this we get that $\gamma_{*}([C])=2\left[\mathcal{E}^{p}(X)\right]$ in $A^{*}\left(\left(\mathbb{P}^{3}\right)^{*}\right)$.

We can compute the class $[C]$ in $A^{1}(X)$ as follows. Let us denote by $h^{*} \in$ $A^{1}\left(\left(\mathbb{P}^{3}\right)^{*}\right)$ the class of a hyperplane in $\left(\mathbb{P}^{3}\right)^{*}$, and by $e^{*} \in A^{1}(X)$ the pullback of $h^{*}$ under the Gauss map $\gamma$. Since $e \in A^{1}(X)$ (which we defined as the pullback of the hyperplane class $h \in A^{1}\left(\mathbb{P}^{3}\right)$ under the inclusion $\left.j: X \hookrightarrow \mathbb{P}^{3}\right)$ generates $A^{1}(X)$, we know that $e^{*}=\alpha e$ for some $\alpha \in \mathbb{Z}$. The push-pull formula implies

$$
\begin{aligned}
\gamma_{*}\left(\left(e^{*}\right)^{2}\right) & =\gamma_{*}\left(\gamma^{*}\left(\left(h^{*}\right)^{2}\right) \cdot[X]\right)=\left(h^{*}\right)^{2} \gamma_{*}([X])=\left(h^{*}\right)^{2} \cdot \operatorname{deg}\left(X^{\vee}\right) h^{*} \\
& =\left(h^{*}\right)^{2} \cdot d(d-1)^{2} h^{*}=d(d-1)^{2} *, \\
j_{*}\left(\left(e^{*}\right)^{2}\right) & =\alpha^{2} j_{*}\left(e^{2}\right)=\alpha^{2} j_{*}\left(j^{*}\left(h^{2}\right) \cdot[X]\right)=\alpha^{2} h^{2} j_{*}([X])=\alpha^{2} h^{2} \cdot d h=\alpha^{2} d *,
\end{aligned}
$$

and thus $\alpha=d-1$. Hence, we have $(d-1) \gamma_{*}(e)=\gamma_{*}\left(e^{*}\right)=d(d-1)^{2}\left(h^{*}\right)^{2}$ and $\gamma_{*}(e)=d(d-1)\left(h^{*}\right)^{2}$. Writing $[C]=\delta e$ for $\delta \in \mathbb{Z}$, we finally derive

$$
2 \operatorname{deg}\left(\mathcal{E}^{p}(X)\right)\left(h^{*}\right)^{2}=2\left[\mathcal{E}^{p}(X)\right]=\gamma_{*}([C])=\delta \gamma_{*}(e)=\delta d(d-1)\left(h^{*}\right)^{2}
$$

and $\delta=(d-2)\left(d^{3}-d^{2}+d-12\right)$ by Proposition 6.20 .

The numbers $a$ and $b$ of cusps and nodes of the curve $C$ are given by [77. Prop. 4.15]:

$$
\begin{aligned}
& a=4 d(d-2)(d-3)\left(d^{3}+3 d-16\right), \\
& b=\frac{1}{2} d(d-2)\left(d^{7}-4 d^{6}+7 d^{5}-45 d^{4}+114 d^{3}-111 d^{2}+548 d-960\right) .
\end{aligned}
$$

Since the curve $C$ does not have any other singularities, we can apply the intersection theoretic genus formula: the geometric genus of $C$ is given by the number of points in the zero-dimensional cycle $\frac{1}{2}\left([C]^{2}+K_{X}[C]\right)$ plus the number $1-a-b$ (cf. [34, Sec. 2.4.6]), where $K_{X}=(d-4) e$ is the canonical class (cf. [34, Sec. 1.4.3]):

$$
\begin{aligned}
j_{*}\left([C]^{2}+K_{X}[C]\right) & =\left(\delta^{2}+\delta(d-4)\right) j_{*}\left(e^{2}\right)=\left(\delta^{2}+\delta(d-4)\right) d * \quad \text { and } \\
\operatorname{genus}(C) & =\frac{1}{2}\left(\delta^{2}+\delta(d-4)\right) d+1-a-b \\
& =3 d^{6}-15 d^{5}+27 d^{4}-104 d^{3}+340 d^{2}-336 d+1 .
\end{aligned}
$$


The map $\gamma^{\prime}: C^{\prime} \rightarrow \mathcal{E}^{p}(X)^{\prime}$ between the normalizations of $C$ and $\mathcal{E}^{p}(X)$ is exactly ramified at the godrons, i.e., the points of tangency of the parabolic curve $P$ and the flecnodal curve $F$ on $X$ (see [81, pp. 229-231]). Thus, the number of those points is the number of points in the zero-dimensional cycle $\frac{1}{2}[P][F]=$ $\frac{1}{2} \cdot 4(d-2) e \cdot(11 d-24) e$, which is

$$
\frac{1}{2} j_{*}([P][F])=2(d-2)(11 d-24) j_{*}\left(e^{2}\right)=2 d(d-2)(11 d-24) * .
$$

We find the genus of $\mathcal{E}^{p}(X)$ by applying the Riemann-Hurwitz formula [50, Cor. 2.4] to $\gamma^{\prime}$ :

$$
2 \operatorname{genus}(C)-2=2\left(2 \operatorname{genus}\left(\mathcal{E}^{p}(X)\right)-2\right)+2 d(d-2)(11 d-24) .
$$

Thus, the genus of $\mathcal{E}^{p}(X)$ is $\frac{1}{2}\left(3 d^{6}-15 d^{5}+27 d^{4}-115 d^{3}+386 d^{2}-384 d+2\right)$. Finally, the degree of the surface $\mathcal{E}(X)=\left(\mathcal{E}^{p}(X)\right)^{\vee}$ is the degree of the tangential surface of the curve $\mathcal{E}^{p}(X)$. The latter degree equals $2\left(\operatorname{deg}\left(\mathcal{E}^{p}(X)\right)+\right.$ $\left.\operatorname{genus}\left(\mathcal{E}^{p}(X)\right)-1\right)-a=d(d-2)(d-3)\left(d^{2}+2 d-4\right)$ (see [79, Thm. 3.2]), since $a$ is also the number of cusps on $\mathcal{E}^{p}(X)$.

Proving the degrees of $\mathcal{C}(X)$ and $\mathcal{T}(X)$ is more technical. We will not include this here. One method is Colley's multiple point theory [25, 26]. Alternatively, one can write $\mathcal{C}(X)$ and $\mathcal{T}(X)$ as the intersection of loci of (inflectional) tangents in the fiber product $\Phi \times_{\mathrm{Gr}} \Phi$ or $\Phi \times_{\mathrm{Gr}} \Phi \times_{\mathrm{Gr}} \Phi$ and remove extra components in the intersection by blowing these up.

\subsection{Computing Visual Events}

Theorem 6.19 gives the degrees of the irreducible components of the visual event surface when $X$ is a general surface of degree $d$ in $\mathbb{P}^{3}$. Table 6.2 below summarizes these degrees for $d \leq 7$. One notices that the degrees are now much larger than those for curves in Table 6.1.

\begin{tabular}{cccccc}
\hline$d$ & $\operatorname{deg}(\mathcal{F}(X))$ & $\operatorname{deg}(\mathcal{C}(X))$ & $\operatorname{deg}(\mathcal{T}(X))$ & $\operatorname{deg}(\mathcal{E}(X))$ & $\operatorname{deg}(\mathcal{P}(X))$ \\
\hline 3 & 0 & 0 & 0 & 0 & 30 \\
4 & 80 & 0 & 0 & 160 & 128 \\
5 & 260 & 510 & 0 & 930 & 330 \\
6 & 576 & 2448 & 624 & 3168 & 672 \\
7 & 1064 & 7308 & 3808 & 8260 & 1190 \\
\hline
\end{tabular}

Table 6.2: Degrees of the components of the visual event surface of a general surface.

The degrees in Table 6.2 pose a challenge because a homogeneous polynomial in four unknowns of degree $\delta$ can have as many as $\left(\begin{array}{c}\delta+3 \\ 3\end{array}\right)$ terms. For instance, if $X$ is a quintic surface, then its flecnodal surface $\mathcal{F}(X)$ has degree $\delta=260$, so the 
expected number of terms is $\left(\begin{array}{c}\delta+3 \\ 3\end{array}\right)=2997411$. In this section, we address this challenge. See Example 6.26 for a solution.

Throughout this section, we make use of the multiple root loci for binary forms. The ideals of these varieties are defined by homogeneous polynomials in the coefficients $c_{0}, c_{1}, \ldots, c_{d}$ of

$$
c_{0} t^{d}+c_{1} t^{d-1}+c_{2} t^{d-2}+\cdots+c_{d-1} t+c_{d} .
$$

For a partition $\lambda=\left(\lambda_{1}, \ldots, \lambda_{k}\right) \in \mathbb{Z}_{>0}^{k}$ with $\sum_{i=1}^{k} \lambda_{i} \leq d$, we write $\Delta_{\lambda}(d)$ for the homogeneous prime ideal in $\mathbb{R}\left[c_{0}, \ldots, c_{d}\right]$ whose variety consists of polynomials (6.18) that have $k$ complex roots with multiplicities $\lambda_{1}, \ldots, \lambda_{k}$. The varieties are called multiple root loci or coincident root loci [71, 70]. For example, $\Delta_{(4)}(d)$ is the prime ideal for polynomials of degree $d$ with one quadruple root.

Example 6.25. Let $d=4$ in $(6.18)$ and consider univariate quartics that have a single root of multiplicity four. These quartics are the points on a rational normal curve in $\mathbb{P}^{4}$. The prime ideal of this curve is $\Delta_{(4)}(4)$. It is generated by the six $2 \times 2$-minors of the $2 \times 4$-matrix

$$
\left[\begin{array}{cccc}
12 c_{0} & 3 c_{1} & 2 c_{2} & 3 c_{3} \\
3 c_{1} & 2 c_{2} & 3 c_{3} & 12 c_{4}
\end{array}\right]
$$

The variety of quintics $(d=5)$ with one root of multiplicity four is the tangential surface of the rational normal curve in $\mathbb{P}^{5}$. Its ideal is the complete intersection of three quadrics:

$\Delta_{(4)}(5)=\left\langle 20 c_{0} c_{4}-8 c_{1} c_{3}+3 c_{2}^{2}, 50 c_{0} c_{5}-6 c_{1} c_{4}+c_{2} c_{3}, 20 c_{1} c_{5}-8 c_{2} c_{4}+3 c_{3}^{2}\right\rangle$.

We give Macaulay2 code for computing $\Delta_{(4)}(4)$ and $\Delta_{(4)}(5)$ using subresultants as described below in Code $\mathrm{K}$ in the Appendix.

Another multiple root locus was seen in Example 6.12. The ideal $\Delta_{(2,2)}(5)$ is minimally generated by ten quintics. We used this to compute the edge surface of a curve with degree five.

We refer to [71, Table 1] for details on the ideals $\Delta_{\lambda}(d)$. Some relevant instances are listed in Table 6.3. Its entries are copied from [71, Table 1]. For instance, the entry $6^{10}, 8^{38}$ in the last column means that $\Delta_{(3,2)}(7)$ is minimally generated by 10 sextics and 38 octics.

The ideals $\Delta_{\lambda}(d)$ can be computed either by direct implicitization, or by using subresultants [1]. The $i$-th subresultant $S_{i}\left(h_{1}, h_{2}\right)$ of two polynomials $h_{1}(t)$ and $h_{2}(t)$ is a polynomial of degree at most $i$ whose coefficients are the determinants of particular minors of the Sylvester matrix of $h_{1}$ and $h_{2}$. The vanishing of $S_{i}\left(h_{1}, h_{2}\right)$ for $0 \leq i \leq d-1$ means that the greatest common divisor (GCD) of $h_{1}$ and $h_{2}$ has degree at least $d$. Moreover, if $S_{d}\left(h_{1}, h_{2}\right)$ is not zero, it is exactly this GCD. If we let $h_{d}$ be the polynomial (6.18) and $h_{d}^{\prime}$ be its derivative with respect to $t$, then the condition that $h_{d}$ has roots with multiplicity $\lambda=\left(\lambda_{1}, \ldots, \lambda_{k}\right)$ is equivalent to the fact that the GCD of $h_{d}$ and $h_{d}^{\prime}$ has degree $\sum_{i=1}^{k}\left(\lambda_{i}-1\right)$ and 
has roots with multiplicities $\lambda^{\prime}=\left(\lambda_{1}-1, \ldots, \lambda_{k}-1\right)$. This allows us to compute the ideal $\Delta_{\lambda}(d)$ recursively.

For example, let us assume that we have recovered the conditions for a polynomial to have a double root $(\lambda=(2))$ and a triple $\operatorname{root}(\lambda=(3))$ for all degrees up to $d-1$. We consider the ideal $I$ defined by $S_{0}\left(h_{d}, h_{d}^{\prime}\right), S_{1}\left(h_{d}, h_{d}^{\prime}\right)$ and $S_{2}\left(h_{d}, h_{d}^{\prime}\right)=0$, saturated by the leading coefficient $c_{0}$. This ideal decomposes into three components corresponding to $\Delta_{(4)}(d), \Delta_{(3,2)}(d)$ and $\Delta_{(2,2,2)}(d)$. The component $\Delta_{(4)}(d)$ can be recovered by adding to $I$ the conditions for $S_{3}\left(h_{d}, h_{d}^{\prime}\right)$ to have a triple root. The component $\Delta_{(3,2)}(d)$, is obtained by adding $I$ the conditions for $S_{3}\left(h_{d}, h_{d}^{\prime}\right)$ to have a double root, and saturating by the condition for it to have a triple root. The component $\Delta_{(2,2,2)}(d)$ is obtained by saturating $I$ for the condition that $S_{3}\left(h_{d}, h_{d}^{\prime}\right)$ has a double root.

\begin{tabular}{cccccc}
\hline Ruled surface & Partition & $d=4$ & $d=5$ & $d=6$ & $d=7$ \\
\hline $\mathcal{F}(X)$ & $\lambda=(4)$ & $2^{6}$ & $2^{3}$ & $2^{1}, 3^{3}, 4^{1}$ & $4^{20}$ \\
$\mathcal{C}(X)$ & $\lambda=(3,2)$ & & $4^{28}$ & $4^{1}, 5^{3}, 6^{31}$ & $6^{10}, 8^{38}$ \\
$\mathcal{T}(X)$ & $\lambda=(2,2,2)$ & & & $4^{45}$ & $6^{78}$ \\
\hline
\end{tabular}

Table 6.3: The ideals $\Delta_{(\lambda)}(d)$ of multiple root loci relevant for visual events of surfaces.

In what follows we assume that the ideals $\Delta_{\lambda}(d)$ have been pre-computed for $d \leq 5$. We use these data to compute the curves $\mathcal{F}^{\ell}(X), \mathcal{C}^{\ell}(X)$ and $\mathcal{T}^{\ell}(X)$ in the Grassmannian $\operatorname{Gr}\left(1, \mathbb{P}^{3}\right)$. The correspondence between the three multi-local events $\mathcal{F}, \mathcal{C}, \mathcal{T}$ and the three special partitions $\lambda$ was seen on the right side in Figure 6.3, where $\lambda$ was denoted by $m$.

Let $f=f\left(x_{0}, x_{1}, x_{2}, x_{3}\right)$ be the polynomial of degree $d$ that defines the surface $X$. We parametrize the line in $\mathbb{P}^{3}$ with Plücker coordinates $q$ using a parameter $t$. For instance, we can write $(6.2)$ dually as $z(t)=\left(q_{01}: t q_{01}: t q_{02}-q_{12}\right.$ : $\left.t q_{03}-q_{13}\right)$. We substitute $z(t)$ into the polynomial $f$, and we regard $f(z(t))$ as a univariate polynomial in $t$, written as in (6.18). The coefficients $c_{i}$ are now homogeneous expressions of degree $d$ in the Plücker coordinates $q$. At this point, we substitute these expressions $c_{i}(q)$ into the generators of $\Delta_{\lambda}(d)$. The result is an ideal in the Plücker coordinates $q$ that defines the desired curve settheoretically. The same method can be applied when local coordinates on the Grassmannian $\operatorname{Gr}\left(1, \mathbb{P}^{3}\right)$ are preferred. In this case, we parametrize the line in $\mathbb{P}^{3}$ by $z(t)=(1: t: \alpha+t \gamma: \beta+t \delta)$.

Example 6.26. Let $d=5$ and consider the smooth quintic surface $X$ defined by

$$
f=x_{0}^{5}+x_{1}^{5}+x_{2}^{5}+x_{3}^{5}+\left(x_{0}+x_{1}+x_{2}+x_{3}\right)^{5}+x_{0} x_{1} x_{2} x_{3}\left(x_{0}+x_{1}+x_{2}+x_{3}\right) .
$$

We compute the curve $\mathcal{F}^{\ell}(X)$ in $\operatorname{Gr}\left(1, \mathbb{P}^{3}\right)$ that represents the flecnodal surface. Its prime ideal has degree 260 and is generated by ten sextics plus the Plücker quadric. This computation was done with the method above, starting from the ideal $\Delta_{(4)}(5)$ in 6.19$)$; see Code $\mathrm{L}$ in the Appendix. 
Let us shift gears and focus on the events $\mathcal{P}$ and $\mathcal{E}$, seen on the left in Figure 6.3. We start with the parabolic surface $\mathcal{P}(X)$. Let $X$ be defined by a polynomial $f \in \mathbb{R}\left[x_{0}, x_{1}, x_{2}, x_{3}\right]$. The ideal $I(P)$ of the parabolic curve $P$ is defined by $f$ and the determinant of the Hessian matrix $H_{f}$. Consider the incidence variety of the parabolic curve and its tangent planes, that is $\left\{\left(x, \mathbb{T}_{X, x}^{\vee}\right) \mid x \in P\right\}$ in $\mathbb{P}^{3} \times\left(\mathbb{P}^{3}\right)^{*}$. We compute the ideal of the incidence variety by adding the

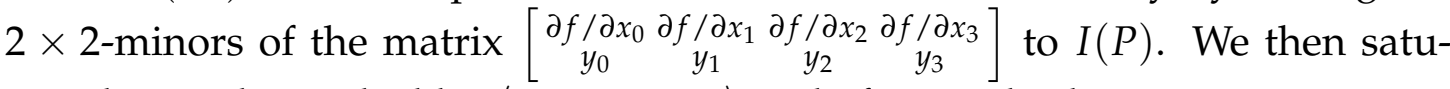
rate the resulting ideal by $\left\langle x_{0}, x_{1}, x_{2}, x_{3}\right\rangle$ and afterwards eliminate $x_{0}, x_{1}, x_{2}, x_{3}$. This furnishes the ideal of the dual curve $\mathcal{P}^{p}(X)$ in $\left(\mathbb{P}^{3}\right)^{*}$, which encodes the developable surface $\mathcal{P}(X)$.

Proposition 6.27. If $X$ is a general cubic surface, the curve $\mathcal{P}^{p}(X)$ is a complete intersection of a quartic and a sextic, obtained from the two basic invariants of ternary cubics.

Proof. A classical fact from invariant theory states that the ring of invariants for ternary cubics is generated by a quartic and a sextic, and these vanish precisely when the cubic has a cusp. We represent $X$ as the blow-up of $\mathbb{P}^{2}$ at six points, namely as the image of the map to $\mathbb{P}^{3}$ defined by four independent cubics $f_{0}, f_{1}, f_{2}, f_{3}$ in $x, y, z$ that vanish at these points. We now consider the cubic $y_{0} f_{0}+y_{1} f_{1}+y_{2} f_{2}+y_{3} f_{3}$, where $y_{0}, y_{1}, y_{2}, y_{3}$ are unknowns. Plugging this cubic into the two basic invariants gives the condition for a plane to meet $X$ in a cuspidal curve. Hence that locus in $\left(\mathbb{P}^{3}\right)^{*}$ is the complete intersection of a quartic and a sextic.

For a general parabolic point $x$ of $X$, the Hessian matrix $H_{f}(x)$ has rank three. Its kernel represents a unique point $p_{x}$ in $\mathbb{P}^{3}$. We use the following simple fact to compute $\mathcal{P}^{\ell}(X)$.

Lemma 6.28. For a general parabolic point $x$ of $X$, the points $p_{x}$ and $x$ span the unique inflectional line of $X$ at $x$.

Proof. The relation $x H_{f}(x) p_{x}^{T}=0$ holds. Euler's relation shows that $x H_{f}(x)$ is the gradient vector of $f$ at $x$. Hence $p_{x}$ lies on the tangent plane to $X$ at $x$. Furthermore, $p_{x}$ belongs to the inflectional line since $p_{x} H_{f}(x) p_{x}^{T}$ is zero. Since $X$ is smooth, the kernel of $H_{f}(x)$ does not contain $x$. Hence $x$ and $p_{x}$ span the inflectional line.

The curve $\mathcal{P}^{\ell}(X) \subset \operatorname{Gr}\left(1, \mathbb{P}^{3}\right)$ is the collection of the lines spanned by a general parabolic point $x$ and the corresponding point $p_{x}$ from Lemma 6.28. This allows us to compute the ideal of $\mathcal{P}^{\ell}(X)$ in dual Plücker coordinates $q_{01}, \ldots, q_{23}$. First, we form the ideal $I$ of the incidence variety $\left\{(x, y) \mid x \in P, y \in \operatorname{ker} H_{f}(x)\right\}$ by adding the four entries of the column vector $H_{f}(x) \cdot y$ to the ideal $I(P)=$ $\left\langle f\right.$, det $\left.H_{f}(x)\right\rangle$. Secondly, we consider the map from the coordinate ring of the Grassmannian to the quotient ring of $I$ that maps Plücker coordinates $q_{i j}$ to the $2 \times 2$-minors of $\left[\begin{array}{llll}x_{0} & x_{1} & x_{2} & x_{3} \\ y_{0} & y_{1} & y_{2} & y_{3}\end{array}\right]$. The kernel of this ring map is the ideal of the curve $\mathcal{P}^{\ell}(X) \subset \operatorname{Gr}\left(1, \mathbb{P}^{3}\right)$. This ideal is generated by four cubics and six quartics, plus 
the Plücker quadric. One verifies computationally that the ideal defines a curve of degree 30 in $\mathbb{P}^{5}$ for a general cubic surface $X$.

Example 6.29. Consider the Fermat cubic $X$ defined by $f=x_{0}^{3}+x_{1}^{3}+x_{2}^{3}+x_{3}^{3}$. We can easily compute the ideal of the curve $\mathcal{P}^{\ell}(X)$ as described above (see Code $\mathrm{M}$ in the Appendix), and from this we find the parabolic surface $\mathcal{P}(X)$. It decomposes into irreducible components of low degree:

$$
\begin{aligned}
& \left(x_{0}+x_{1}\right) \cdot\left(x_{0}+x_{2}\right) \cdot\left(x_{0}+x_{3}\right) \cdot\left(x_{1}+x_{2}\right) \cdot\left(x_{1}+x_{3}\right) \cdot\left(x_{2}+x_{3}\right) \\
& \cdot\left(x_{0}^{2}-x_{0} x_{1}+x_{1}^{2}\right) \cdot\left(x_{0}^{2}-x_{0} x_{2}+x_{2}^{2}\right) \cdot\left(x_{0}^{2}-x_{0} x_{3}+x_{3}^{2}\right) \\
& \cdot\left(x_{1}^{2}-x_{1} x_{2}+x_{2}^{2}\right) \cdot\left(x_{1}^{2}-x_{1} x_{3}+x_{3}^{2}\right) \cdot\left(x_{2}^{2}-x_{2} x_{3}+x_{3}^{2}\right) \\
& \cdot\left(x_{1}^{3}+x_{2}^{3}+x_{3}^{3}\right) \cdot\left(x_{0}^{3}+x_{2}^{3}+x_{3}^{3}\right) \cdot\left(x_{0}^{3}+x_{1}^{3}+x_{3}^{3}\right) \cdot\left(x_{0}^{3}+x_{1}^{3}+x_{2}^{3}\right) .
\end{aligned}
$$

This is one of the few cases where symbolic computation of the equation of $\mathcal{P}(X)$ is easy.

Example 6.30. Fix the cubic $f=x_{0}^{3}+x_{1}^{3}+x_{2}^{3}+x_{3}^{3}+\left(x_{0}+2 x_{1}+3 x_{2}+4 x_{3}\right)^{3}$. It defines our surface $X$. Using the method above, we rapidly compute the ideal of $\mathcal{P}^{\ell}(X)$. We demonstrate how to find the visual events of type $\mathcal{P}$ as the camera moves along a line; see Code $\mathrm{N}$ in the Appendix.

We consider the fixed line in $\mathbb{P}^{3}$ with the parametric representation $z(t)=$ $(t: 1: t-1: t+1)$. Let $Q$ be the skew-symmetric $4 \times 4$-matrix obtained from (6.1) by substituting to dual Plücker coordinates. We add the four coordinates of $z(t) \cdot Q$ to the ideal of $\mathcal{P}^{\ell}$, we then saturate with respect to $\left\langle q_{01}, \ldots, q_{23}\right\rangle$, and thereafter we eliminate the unknowns $q_{i j}$. The result is

$$
\begin{gathered}
495403946635821355157683145728 t^{30}+4349505253226024309192581220352 t^{29} \\
+18437739306679654261938338946432 t^{28}+50562321054013553614808463278912 t^{27} \\
+\cdots \cdots-81509153943200707008 t^{2}-1885273424647073088 t-19650742648215232 .
\end{gathered}
$$

This polynomial has 30 distinct complex roots. Precisely eight of them are real:

$$
\begin{aligned}
& \{-1.01358602985259,-1.011352289518,-0.600974923580648,-0.35014676100811, \\
& -0.2668550692437,-0.191676056625,-0.0811161566932513,0.378943747730770\} .
\end{aligned}
$$

These eight roots mark the visual events of type $\mathcal{P}$ as the viewpoint travels along the line $z(t)$.

The univariate polynomial of degree 30 can also be computed from the dual curve $\mathcal{P}^{p}$. Let $g_{1}$ and $g_{2}$ be the polynomials in $y_{0}, y_{1}, y_{2}, y_{3}$ of degree four and six promised in Proposition 6.27. We augment the ideal $I\left(\mathcal{P}^{p}\right)=\left\langle g_{1}, g_{2}\right\rangle$ by the $3 \times 3$-minors of the $3 \times 4$-matrix

$$
\left[\begin{array}{cccc}
\partial g_{1} / \partial x_{0} & \partial g_{1} / \partial x_{1} & \partial g_{1} / \partial x_{2} & \partial g_{1} / \partial x_{3} \\
\partial g_{2} / \partial x_{0} & \partial g_{2} / \partial x_{1} & \partial g_{2} / \partial x_{2} & \partial g_{2} / \partial x_{3} \\
t & 1 & t-1 & t+1
\end{array}\right]
$$

We then saturate the resulting ideal by the ideal of the six $2 \times 2$-minors in first 
two rows, and finally we eliminate $x_{0}, x_{1}, x_{2}, x_{3}$. This gives the same polynomial of degree 30 in $t$.

We found that the computation of the edge surface $\mathcal{E}(X)$ is more challenging than that of the parabolic surface $\mathcal{P}(X)$. Consider the case when $X$ is a general quartic. Here, the surface $\mathcal{E}(X)$ has degree 160, and hence so does the curve $\mathcal{E}^{\ell}(X)$ in $\operatorname{Gr}\left(1, \mathbb{P}^{3}\right)$. We succeeded in computing the ideal of this curve only for quartics $X$ that are singular or very special. For instance, if $X$ is the Fermat quartic then $\mathcal{E}(X)$ is a surface of degree 80 , with multiplicity two. Since $\mathcal{E}(X)$ is developable, we could also try to use $\mathcal{E}^{p}(X)$ as an encoding. Unfortunately, the degree is then even higher. Namely, by Proposition 6.20, the dual curve $\mathcal{E}^{p}(X)$ has degree 480 in $\left(\mathbb{P}^{3}\right)^{*}$ for a general quartic $X$. The computation of edge surfaces $\mathcal{E}(X)$ definitely requires further research.

So far we have studied coisotropic varieties in general as well as in examples and applications. In the next chapter, we will switch gears and introduce the study of isotropic varieties. In particular, this will lead us to a classification of congruences, in which all congruences from Chapters 5 and 6 appear again (see Table 7.2). 



\section{Isotropic Varieties}

This chapter is based on an ongoing collaboration with James Mathews. We investigate a dual notion to coisotropic varieties. Instead of imposing rank one conditions on the conormal spaces of a subvariety of a Grassmannian, we require such conditions to hold for its tangent spaces.

Definition 7.1. An irreducible subvariety $\Sigma \subset \operatorname{Gr}\left(\ell, \mathbb{P}^{n}\right)$ of dimension $d \geq 1$ is isotropic if, for every $L \in \operatorname{Reg}(\Sigma)$, the tangent space of $\Sigma$ at $L$ is spanned by rank one homomorphisms or is in the Zariski closure of the set of such spaces, i.e.,

$$
\mathbb{P}\left(T_{\Sigma, L}\right) \in \operatorname{Sec}_{d-1}\left(\operatorname{Seg}\left(\boldsymbol{L}, \mathbb{A}^{n+1} / \boldsymbol{L}\right)\right) .
$$

Moreover, $\Sigma$ is strongly isotropic if, for every $L \in \operatorname{Reg}(\Sigma)$, the rank of every homomorphism in the tangent space of $\Sigma$ at $L$ is at most one, i.e.,

$$
\mathbb{P}\left(T_{\Sigma, L}\right) \subset \operatorname{Seg}\left(\boldsymbol{L}, \mathbb{A}^{n+1} / \boldsymbol{L}\right) .
$$

Example 7.2. Both definitions of isotropy agree for curves. Let us consider a first example of an isotropic curve: given an irreducible curve $C \subset \mathbb{P}^{3}$ of degree at least two, the curve $\mathcal{T}^{\ell}(C) \subset \operatorname{Gr}\left(1, \mathbb{P}^{3}\right)$ consisting of the tangent lines to $C$ (cf. Figure 6.2) is isotropic by Example 2.8 .

Dually to Corollary 4.5, we observe that all subvarieties of Grassmannians with low enough codimension are isotropic.

Lemma 7.3. Every subvariety of $\operatorname{Gr}\left(\ell, \mathbb{P}^{n}\right)$ of codimension at most $n-1$ is isotropic.

Proof. Let $\Sigma \subset \operatorname{Gr}\left(\ell, \mathbb{P}^{n}\right)$ be a subvariety with $\operatorname{codim} \Sigma \leq n-1$. The dimension $d$ of $\Sigma$ is at least $(\ell+1)(n-\ell)-(n-1)=\ell(n-\ell-1)+1$. Moreover, the codimension of $\operatorname{Seg}\left(\boldsymbol{L}, \mathbb{A}^{n+1} / \boldsymbol{L}\right)$ in $\mathbb{P}\left(\operatorname{Hom}\left(\boldsymbol{L}, \mathbb{A}^{n+1} / \boldsymbol{L}\right)\right)$ is $\ell(n-\ell-1)$ for every $L \in \operatorname{Gr}\left(\ell, \mathbb{P}^{n}\right)$. By Lemma 4.1, we have

$$
\operatorname{Sec}_{d-1}\left(\operatorname{Seg}\left(\boldsymbol{L}, \mathbb{A}^{n+1} / \boldsymbol{L}\right)\right)=\operatorname{Gr}\left(d-1, \mathbb{P}\left(\operatorname{Hom}\left(\boldsymbol{L}, \mathbb{A}^{n+1} / \boldsymbol{L}\right)\right)\right) .
$$

In particular, $\Sigma$ is isotropic.

Example 7.4. All surfaces and hypersurfaces in $\mathrm{Gr}\left(1, \mathbb{P}^{3}\right)$ are isotropic.

Example 7.5. The secant congruence $\operatorname{Sec}(C)$ of a nondegenerate irreducible space curve is not strongly isotropic. Using the notation in Example 4.4 we see that the tangent space of $\operatorname{Sec}(C)$ at a general secant line $L$ is spanned by two rank one homomorphisms $\varphi_{1}, \varphi_{2}: \boldsymbol{L} \rightarrow \mathbb{A}^{4} / \boldsymbol{L}$ with $\operatorname{ker} \varphi_{1}=\boldsymbol{x}_{\mathbf{1}}, \operatorname{im} \varphi_{1}=\boldsymbol{H}_{\mathbf{2}} / \boldsymbol{L}$, 
$\operatorname{ker} \varphi_{2}=\boldsymbol{x}_{\mathbf{2}}$, and $\operatorname{im} \varphi_{2}=\boldsymbol{H}_{\mathbf{1}} / \boldsymbol{L}$. Thus, $\operatorname{Sec}(C)$ is isotropic, but since $L$ was chosen generally, we have $\operatorname{rank}\left(\varphi_{1}+\varphi_{2}\right)=2$ and $\operatorname{Sec}(C)$ is not strongly isotropic.

As before, our leading question will be if we can characterize (strongly) isotropic varieties by underlying projective varieties. By Example 7.4, it is enough to consider isotropic curves to classify all isotropic subvarieties of $\operatorname{Gr}\left(1, \mathbb{P}^{3}\right)$. We give a full characterization of isotropic curves in Section 7.1. As for the stronger notion of coisotropy, it is not too hard to determine all strongly isotropic varieties. We provide this classification in Section 7.2. Finally, Examples 7.4 and 4.6 show that all congruences in $\operatorname{Gr}\left(1, \mathbb{P}^{3}\right)$ are isotropic and coisotropic at the same time. We can actually classify all congruences by their underlying projective varieties. We present these results in Section 7.3. Hence, we conclude with the following open question:

Can we characterize all subvarieties of Grassmannians which are isotropic and coisotropic at the same time by underlying projective varieties?

\subsection{Isotropic Curves}

In this section, we show that the isotropic curves are exactly the curves of osculating spaces to projective curves. For a detailed treatment of osculating spaces and osculating bundles of projective curves we refer to [79]. We only give a brief and non-exhaustive summary here. For a nondegenerate irreducible curve $C \subset \mathbb{P}^{n}$ and an integer $0 \leq k \leq n$, we define the osculating $k$-space $L_{k}(x)$ at a point $x \in \operatorname{Reg}(C)$ to be the $k$-dimensional subspace of $\mathbb{P}^{n}$ which has the highest order of contact with $C$ at $x$. This order of contact of $L_{k}(x)$ with $C$ is at least $k+1$, and at a general point $x \in C$ it is exactly $k+1$. Those osculating $k$-spaces with a higher contact order are called hyperosculating or stationary $k$-spaces. For example, the tangent line at a flex point is a stationary tangent. For $k<n$, we define the curve $\operatorname{Osc}_{k}(C) \subset \operatorname{Gr}\left(k, \mathbb{P}^{n}\right)$ of osculating $k$-spaces of $C$ as the Zariski closure of the set of all osculating $k$-spaces $L_{k}(x)$ at smooth points $x \in C$.

Remark 7.6. Clearly, $\operatorname{Osc}_{0}(C)=C$. The curve $\mathrm{Osc}_{1}(C)$ consists of the tangent lines of $C$. It was denoted by $\mathcal{T}^{\ell}(C)$ for $C \subset \mathbb{P}^{3}$ in Chapter 6 as well as Examples 2.8 and 7.2. The curve $\operatorname{Osc}_{n-1}(C) \subset \operatorname{Gr}\left(n-1, \mathbb{P}^{n}\right) \cong\left(\mathbb{P}^{n}\right)^{*}$ is also known as the dual curve of $C$. It was denoted by $\mathcal{T}^{p}(C)$ for $C \subset \mathbb{P}^{3}$ in Chapter 6 . The dual curve of $C \subset \mathbb{P}^{3}$ is the edge of regression of the dual surface $C^{\vee}$, or in other words, the dual surface $C^{\vee}$ is the tangent developable of the dual curve of $C$ (see Fig. 6.1). For any nondegenerate curve $C \subset \mathbb{P}^{n}$, the dual curve of $\operatorname{Osc}_{n-1}(C)$ is again the original curve $C$ [79, Thm. 5.1].

Of course we can also define osculating spaces to degenerate curves, which we will use in the formulation of Theorem 7.10. If a curve $C \subset \mathbb{P}^{n}$ spans $\mathbb{P}^{m} \subset$ $\mathbb{P}^{n}$ for $m<n$, we define the osculating $k$-spaces for $0 \leq k \leq m$ inside of $\mathbb{P}^{m}$ such that we have $m$ curves $\operatorname{Osc}_{k}(C) \subset \operatorname{Gr}\left(k, \mathbb{P}^{n}\right)$ for $0 \leq k<m$. 
Now we consider an irreducible isotropic curve $\Sigma \subset \operatorname{Gr}\left(\ell, \mathbb{P}^{n}\right)$. Since each curve in $\mathbb{P}^{n}$ or $\left(\mathbb{P}^{n}\right)^{*}$ is trivially isotropic, we assume $1 \leq \ell \leq n-2$. For each $L \in \operatorname{Reg}(\Sigma)$, the tangent space $T_{\Sigma, L}$ is spanned by a rank one homomorphism $\varphi_{L}: L \rightarrow \mathbb{A}^{n+1} / \boldsymbol{L}$. Hence, to every $L \in \operatorname{Reg}(\Sigma)$ we associate unique linear spaces $L^{-} \in \operatorname{Gr}\left(\ell-1, \mathbb{P}^{n}\right)$ and $L^{+} \in \operatorname{Gr}\left(\ell+1, \mathbb{P}^{n}\right)$ such that $L^{-} \subset L \subset L^{+}$, $\operatorname{ker} \varphi_{L}=L^{-}$, and $\operatorname{im} \varphi_{L}=L^{+} / L$. This provides us with a rational map $\tau: \Sigma \rightarrow \operatorname{Gr}\left(\ell-1, \mathbb{P}^{n}\right) \times \operatorname{Gr}\left(\ell+1, \mathbb{P}^{n}\right)$. We denote the projections of the codomain onto the first or second factor by $\pi^{-}$or $\pi^{+}$, respectively. Moreover, for $\star \in\{+,-\}$, we let $\omega^{\star}:=\pi^{\star} \circ \tau$ and define $\Sigma^{\star} \subset \operatorname{Gr}\left(\ell \star 1, \mathbb{P}^{n}\right)$ as the Zariski closure of the image of $\omega^{\star}$. We summarize these maps in the following commutative diagram:

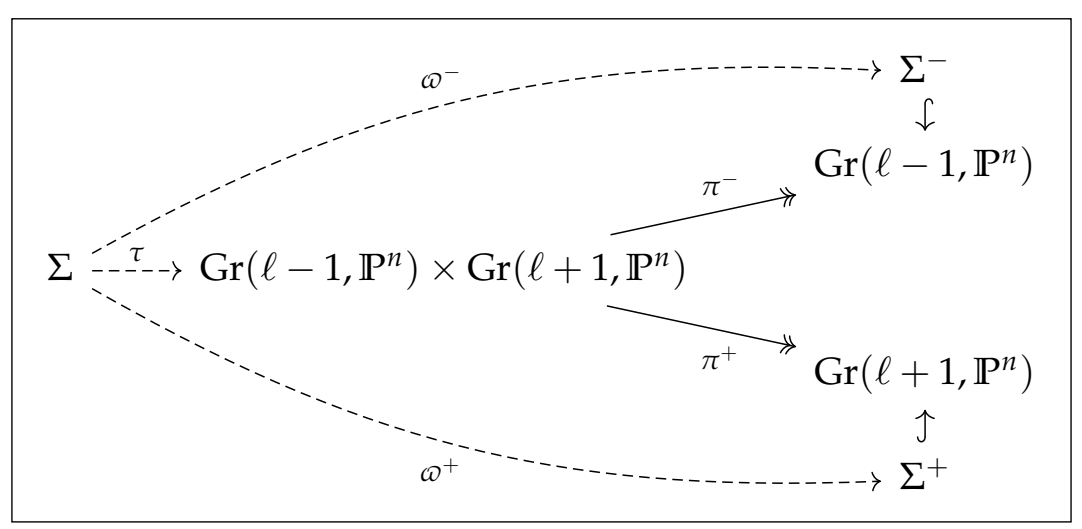

Since $\Sigma$ is an irreducible curve, $\Sigma^{-}$and $\Sigma^{+}$are both irreducible and have each dimension zero or one. If $\Sigma^{-}$is a point, then all $L \in \Sigma$ contain the same $(\ell-1)$-dimensional subspace. Analogously, if $\Sigma^{+}$is a point, then all $L \in \Sigma$ are contained in the same $(\ell+1)$-dimensional projective space. If $\Sigma^{-}$or $\Sigma^{+}$is a curve, we show now that it is isotropic and that we can recover $\Sigma$ from it.

Lemma 7.7. If $\Sigma^{-}$is a curve, then, for each $L \in \operatorname{Reg}(\Sigma)$ such that $L^{-} \in \operatorname{Reg}\left(\Sigma^{-}\right)$, the image of every homomorphism in $T_{\Sigma^{-}, L^{-}}$is contained in $L / L^{-}$. In particular, $\Sigma^{-}$ is isotropic.

Proof. Let $L \in \Sigma$ be general such that the differential $D_{L} \mathcal{W}^{-}: T_{\Sigma, L} \rightarrow T_{\Sigma^{-}, L^{-}}$of $\mathfrak{\omega}^{-}$at $L$ is bijective. For each $\varphi \in T_{\Sigma, L}$, we have $\left.\varphi\right|_{L^{-}}=\left(D_{L^{\prime}} \mathcal{C}^{-}(\varphi) \bmod \boldsymbol{L}\right)$ by Corollary 2.9. Since the kernel of every homomorphism in $T_{\Sigma, L}$ contains $L^{-}$, we have $(\psi \bmod L)=0$ for every $\psi \in T_{\Sigma^{-}, L^{-}}$. Thus, the image of each homomorphism in $T_{\Sigma^{-}, L^{-}}$is contained in $L / L^{-}$.

Lemma 7.8. If $\Sigma^{+}$is a curve, then, for each $L \in \operatorname{Reg}(\Sigma)$ such that $L^{+} \in \operatorname{Reg}\left(\Sigma^{+}\right)$, the kernel of every homomorphism in $T_{\Sigma^{+}, L^{+}}$contains $\boldsymbol{L}$. In particular, $\Sigma^{+}$is isotropic.

Proof. The proof of this statement is completely dual to the proof of Lemma 7.7. We consider a general $L \in \Sigma$ such that the differential $D_{L} \omega^{+}$is bijective. For each $\varphi \in T_{\Sigma, L}$, we have $\left.D_{L} \mathcal{O}^{+}(\varphi)\right|_{\boldsymbol{L}}=\left(\varphi \bmod \boldsymbol{L}^{+}\right)$by Corollary 2.9. Since the image of every homomorphism in $T_{\Sigma, L}$ is contained in $L^{+} / L$, we have that $\boldsymbol{L} \subset \operatorname{ker} \psi$ for every $\psi \in T_{\Sigma^{+}, L^{+}}$. 
For every curve $\Sigma \subset \mathbb{P}^{n}$, we define $\Sigma^{+} \subset \operatorname{Gr}\left(1, \mathbb{P}^{n}\right)$ as above. Note that $\Sigma^{+}$is the curve of tangent lines to $\Sigma$. Dually, for a curve $\Sigma \subset \operatorname{Gr}\left(n-1, \mathbb{P}^{n}\right) \cong\left(\mathbb{P}^{n}\right)^{*}$, we define $\Sigma^{-} \subset \operatorname{Gr}\left(n-2, \mathbb{P}^{n}\right)$. This allows us to formulate the following:

Corollary 7.9. Let $\Sigma \subset \operatorname{Gr}\left(\ell, \mathbb{P}^{n}\right)$ be an irreducible isotropic curve. If $\ell \geq 1$ and $\Sigma^{-}$ is a curve, then $\left(\Sigma^{-}\right)^{+}=\Sigma$. Dually, if $\ell \leq n-2$ and $\Sigma^{+}$is a curve, then $\left(\Sigma^{+}\right)^{-}=\Sigma$.

Proof. The first part follows immediately from Lemma 7.7, which shows that $\left(L^{-}\right)^{+}=L$ for a general $L \in \Sigma$. Similarly, Lemma 7.8 implies the second part.

So far we have shown that we can associate a maximal sequence of isotropic curves in different Grassmannians to each isotropic curve $\Sigma \subset \operatorname{Gr}\left(\ell, \mathbb{P}^{n}\right)$, where $0 \leq \ell \leq n-1$. By this, we mean a sequence of isotropic curves

$$
\left.\begin{array}{ccccccc}
\Sigma_{\ell_{1}} & \Sigma_{\ell_{1}+1}, & \ldots, & \Sigma_{\ell,} & \ldots, & \Sigma_{\ell_{2}-1}, & \Sigma_{\ell_{2}} \\
\cap & \cap & & \cap & & \cap & \cap \\
\operatorname{Gr}\left(\ell_{1}, \mathbb{P}^{n}\right) & \operatorname{Gr}\left(\ell_{1}+1, \mathbb{P}^{n}\right) & & \operatorname{Gr}\left(\ell, \mathbb{P}^{n}\right) & & \operatorname{Gr}\left(\ell_{2}-1, \mathbb{P}^{n}\right) & \operatorname{Gr}\left(\ell_{2}, \mathbb{P}^{n}\right)
\end{array}\right),
$$

where $\Sigma_{\ell}=\Sigma$, and $\ell_{1} \in\{0, \ldots, \ell\}$ is minimal and $\ell_{2} \in\{\ell, \ldots, n-1\}$ is maximal such that $\Sigma_{i}^{+}=\Sigma_{i+1}$ holds for all $\ell_{1} \leq i<\ell_{2}$ and $\Sigma_{j}^{-}=\Sigma_{j-1}$ holds for all $\ell_{1}<j \leq \ell_{2}$.

If $\ell_{2}<n-1$, then $\Sigma_{\ell_{2}}^{+}$is a point in $\operatorname{Gr}\left(\ell_{2}+1, \mathbb{P}^{n}\right)$, i.e., an $\left(\ell_{2}+1\right)$-dimensional subspace $P_{2} \subset \mathbb{P}^{n}$. For each $\ell_{1} \leq i \leq \ell_{2}$, we have that every $L \in \Sigma_{i}$ is contained in $P_{2}$. Hence, the whole sequence (7.1) of isotropic curves can be embedded into Grassmannians of subspaces of $P_{2}$ via $\Sigma_{i} \hookrightarrow \operatorname{Gr}\left(i, P_{2}\right), L \mapsto L$.

Dually, if $\ell_{1}>0$, then $\Sigma_{\ell_{1}}^{-}$is a point in $\operatorname{Gr}\left(\ell_{1}-1, \mathbb{P}^{n}\right)$, i.e., an $\left(\ell_{1}-1\right)$ dimensional subspace $P_{1} \subset \mathbb{P}^{n}$. For each $\ell_{1} \leq i \leq \ell_{2}$, we have that every $L \in \Sigma_{i}$ contains $P_{1}$. Thus, denoting by $\pi_{P_{1}}: \mathbb{P}^{n} \rightarrow \mathbb{P}\left(\mathbb{A}^{n+1} / \boldsymbol{P}_{\mathbf{1}}\right) \cong \mathbb{P}^{n-\ell_{1}}$ the projection away from $P_{1}$, we can embed the whole sequence (7.1) into smaller Grassmannians via $\Sigma_{i} \hookrightarrow \operatorname{Gr}\left(i-\ell_{1}, \mathbb{P}^{n-\ell_{1}}\right), L \mapsto \pi_{P_{1}}(L)$. We denote by $\pi_{P_{1}}\left(\Sigma_{i}\right)$ the image of $\Sigma_{i}$ under this embedding.

Therefore, we can always assume that $\ell_{1}=0$ and $\ell_{2}=n-1$. In this case, $\Sigma_{k}=\operatorname{Osc}_{k}\left(\Sigma_{0}\right)$ for every $0 \leq k \leq n-1$, as the following theorem shows. Recall for this theorem that we view the empty set as a projective space with dimension -1 .

Theorem 7.10. 1. For each irreducible curve $\Sigma_{0} \subset \mathbb{P}^{n}$ and each $0 \leq k \leq \ell_{2}$, we have that $\operatorname{Osc}_{k}\left(\Sigma_{0}\right)=\Sigma_{k}$. In particular, the curve $\operatorname{Osc}_{k}\left(\Sigma_{0}\right)$ of osculating $k$-planes of $\Sigma_{0}$ is isotropic.

2. For each irreducible isotropic curve $\Sigma \subset \operatorname{Gr}\left(\ell, \mathbb{P}^{n}\right)$, there is a unique subspace $P_{1} \subset \mathbb{P}^{n}$ and a unique irreducible curve $C \subset \mathbb{P}^{n-\operatorname{dim} P_{1}-1}$ such that $-1 \leq$ $\operatorname{dim} P_{1}<\ell$, every $L \in \Sigma$ contains $P_{1}$, and $\pi_{P_{1}}(\Sigma)=\operatorname{Osc}_{\ell-\operatorname{dim} P_{1}-1}(C)$.

Proof. Let us first consider the second part of Theorem 7.10. We write $\Sigma_{\ell}=\Sigma$ and associate the maximal sequence (7.1) of isotropic curves. If $\ell_{1}=0$, we set 
$P_{1}:=\varnothing$. Otherwise, $\ell_{1}>0$ and $P_{1}:=\Sigma_{\ell_{1}}^{-}$. Note that $P_{1}$ is the maximal subspace of $\mathbb{P}^{n}$ with the property that it is contained in every $L \in \Sigma$. In any case, we define $C:=\pi_{P_{1}}\left(\Sigma_{\ell_{1}}\right) \subset \mathbb{P}^{n-\ell_{1}}$. Now, $\pi_{P_{1}}(\Sigma)=\operatorname{Osc}_{\ell-\ell_{1}}(C)$ follows from the first part of Theorem 7.10.

Hence, it is enough to show the first assertion of this theorem. For this, we consider an irreducible curve $\Sigma_{0} \subset \mathbb{P}^{n}$ and a point $p \in \operatorname{Reg}\left(\Sigma_{0}\right)$. We choose coordinates such that $p=(1: 0: \ldots: 0)$. Without loss of generality, we may work in the affine chart $\mathbb{P}^{n} \backslash Z\left(x_{0}\right)$. Since $\Sigma_{0}$ is smooth around $p$, there is a local analytic isomorphism $f$ from a neighborhood of the origin in $\mathbb{A}^{1}$ to a neighborhood of the point $p$ in $\Sigma_{0}$. The map $f$ has the form $f(t)=\left(f_{1}(t), \ldots, f_{n}(t)\right)$ for some $f_{1}, \ldots, f_{n} \in \mathbb{C} \llbracket t \rrbracket$ with $f(0)=(0, \ldots, 0)$. In our affine chart, the osculating $k$-plane at $f(\varepsilon)$ is the affine span of $f(\varepsilon), \frac{\partial f}{\partial t}(\varepsilon), \ldots, \frac{\partial^{k} f}{\partial^{k} t}(\varepsilon)$. We define

$$
A_{k}(\varepsilon):=\left[\begin{array}{cccc}
1 & f_{1}(\varepsilon) & \cdots & f_{n}(\varepsilon) \\
1 & \frac{\partial f_{1}}{\partial t}(\varepsilon) & \cdots & \frac{\partial f_{n}}{\partial t}(\varepsilon) \\
\vdots & \vdots & & \vdots \\
1 & \frac{\partial^{k} f_{1}}{\partial^{k} t}(\varepsilon) & \cdots & \frac{\partial^{k} f_{n}}{\partial^{k} t}(\varepsilon)
\end{array}\right] \in \mathbb{C}^{(k+1) \times(n+1)}
$$

such that the rowspace $L_{k}(\varepsilon) \in \mathrm{Gr}\left(k, \mathbb{P}^{n}\right)$ of $A_{k}(\varepsilon)$ is an osculating $k$-plane of $\Sigma_{0}$. In particular, $L_{k}(0)$ is the osculating $k$-plane at $p$. The tangent line at $A_{k}(\varepsilon)$ to the curve in $\mathbb{A}^{(k+1) \times(n+1)}$ parametrized by $t \mapsto A_{k}(t)$ is the affine span of $A_{k}(\varepsilon)$ and

$$
\left[\begin{array}{cccc}
0 & \frac{\partial f_{1}}{\partial t}(\varepsilon) & \cdots & \frac{\partial f_{n}}{\partial t}(\varepsilon) \\
\vdots & \vdots & & \vdots \\
0 & \frac{\partial^{k+1} f_{1}}{\partial^{k+1} t}(\varepsilon) & \cdots & \frac{\partial^{k+1} f_{n}}{\partial^{k+1} t}(\varepsilon)
\end{array}\right] .
$$

Hence, by Corollary 2.10, the tangent line at $L_{k}(0)$ to the curve $\operatorname{Osc}_{k}\left(\Sigma_{0}\right)$, which is locally parametrized by $t \mapsto L_{k}(t)$, is spanned by

$$
\begin{aligned}
\varphi: \boldsymbol{L}_{\boldsymbol{k}}(\mathbf{0}) & \longrightarrow \mathbb{A}^{n+1} / \mathbf{L}_{\boldsymbol{k}}(\mathbf{0}), \\
(1, f(0)) & \longmapsto 0+\mathbf{L}_{\boldsymbol{k}}(\mathbf{0}), \\
\left(1, \frac{\partial f}{\partial t}(0)\right) & \longmapsto 0+\boldsymbol{L}_{\boldsymbol{k}}(\mathbf{0}), \\
\vdots & \\
\left(1, \frac{\partial^{k-1} f}{\partial^{k-1} t}(0)\right) & \longmapsto 0+\boldsymbol{L}_{\boldsymbol{k}}(\mathbf{0}), \\
\left(1, \frac{\partial^{k} f}{\partial^{k} t}(0)\right) & \longmapsto\left(0, \frac{\partial^{k+1} f}{\partial^{k+1} t}(0)\right)+\boldsymbol{L}_{\boldsymbol{k}}(\mathbf{0}) .
\end{aligned}
$$

Since $p$ was an arbitrary smooth point of $\Sigma_{0}$, this shows that the curve $\operatorname{Osc}_{k}\left(\Sigma_{0}\right)$ is isotropic with $\left(\operatorname{Osc}_{k}\left(\Sigma_{0}\right)\right)^{+}=\operatorname{Osc}_{k+1}\left(\Sigma_{0}\right)$ and $\left(\operatorname{Osc}_{k}\left(\Sigma_{0}\right)\right)^{-}=\operatorname{Osc}_{k-1}\left(\Sigma_{0}\right)$. Now the assertion $\operatorname{Osc}_{k}\left(\Sigma_{0}\right)=\Sigma_{k}$ follows by induction on $k$. It is trivial for $k=0$. For $k>0$, the induction hypothesis yields $\left(\operatorname{Osc}_{k}\left(\Sigma_{0}\right)\right)^{-}=\operatorname{Osc}_{k-1}\left(\Sigma_{0}\right)=$ $\Sigma_{k-1}$, which then implies $\operatorname{Osc}_{k}\left(\Sigma_{0}\right)=\left(\left(\operatorname{Osc}_{k}\left(\Sigma_{0}\right)\right)^{-}\right)^{+}=\Sigma_{k-1}^{+}=\Sigma_{k}$. 


\begin{tabular}{c|cc}
\hline & $\Sigma^{+} \subset \operatorname{Gr}\left(2, \mathbb{P}^{3}\right)$ curve & $\Sigma^{+} \in \operatorname{Gr}\left(2, \mathbb{P}^{3}\right)$ point \\
& $Y:=\left(\Sigma^{+}\right)^{\perp} \subset\left(\mathbb{P}^{3}\right)^{*}$ & $H:=\Sigma^{+} \subset \mathbb{P}^{3}$ plane \\
\hline$\Sigma^{-} \subset \mathbb{P}^{3}$ curve & $\mathcal{S}=\mathcal{T}\left(\Sigma^{-}\right)$ & $\mathcal{S}=\mathcal{T}\left(\Sigma^{-}\right)$ \\
& $\mathcal{S}^{\vee}=\Upsilon$ & $\Sigma^{-} \subset H=\mathcal{S}$ \\
$\Sigma^{-} \in \mathbb{P}^{3}$ point & $\mathcal{S}$ is cone through $\Sigma^{-}$ & \\
& $\mathcal{S}^{\vee}=Y \subset\left(\Sigma^{-}\right)^{\vee}$ & $\Sigma^{-} \in H=\mathcal{S}$ \\
\hline
\end{tabular}

Table 7.1: All cases of isotropic curves $\Sigma \subset \operatorname{Gr}\left(1, \mathbb{P}^{3}\right)$. The developable surface $\mathcal{S}$ is ruled by the lines on $\Sigma$. See also Figure 6.1.

Example 7.11. Let $\Sigma \subset \operatorname{Gr}\left(1, \mathbb{P}^{3}\right)$ be an irreducible isotropic curve and let $\mathcal{S}$ in $\mathbb{P}^{3}$ be the union of all lines on $\Sigma$. If $\Sigma^{-} \subset \mathbb{P}^{3}$ is a curve, the surface $\mathcal{S}$ is the tangent developable of $\Sigma^{-}$. If $\Sigma^{-}$is a point, then $\mathcal{S}$ is a cone with vertex $\Sigma^{-}$. In any of the two cases, the surface $\mathcal{S}$ can be either a plane or the dual of a curve, as Table 7.1 summarizes. So shortly put, a curve $\Sigma \subset \operatorname{Gr}\left(1, \mathbb{P}^{3}\right)$ is isotropic if and only if the surface ruled by the lines on $\Sigma$ is developable. By Corollary 2.11 . this assertion is equivalent to the second part of Theorem 6.2 .

\subsection{Strongly Isotropic Varieties}

In this section, we show that each strongly isotropic variety is either a curve or a subvariety of an $\alpha$ - or $\beta$-variety.

Definition 7.12. For $1 \leq \ell \leq n$ and $P_{1} \in \operatorname{Gr}\left(\ell-1, \mathbb{P}^{n}\right)$, we call

$$
\alpha\left(P_{1}\right):=\left\{L \in \operatorname{Gr}\left(\ell, \mathbb{P}^{n}\right) \mid P_{1} \subset L\right\}
$$

the $\alpha$-variety of $P_{1}$. Analogously, for $0 \leq \ell \leq n-2$ and $P_{2} \in \operatorname{Gr}\left(\ell+1, \mathbb{P}^{n}\right)$, the $\beta$-variety of $P_{2}$ is

$$
\beta\left(P_{2}\right):=\left\{L \in \operatorname{Gr}\left(\ell, \mathbb{P}^{n}\right) \mid L \subset P_{2}\right\} .
$$

Note that $\alpha\left(P_{1}\right)$ is isomorphic to $\operatorname{Gr}\left(0, \mathbb{P}^{n-\ell}\right)=\mathbb{P}^{n-\ell}$, and that $\beta\left(P_{2}\right)$ is isomorphic to $\operatorname{Gr}\left(\ell, \mathbb{P}^{\ell+1}\right) \cong\left(\mathbb{P}^{\ell+1}\right)^{*}$.

Remark 7.13. The tangent space of the $\alpha$-variety $\alpha\left(P_{1}\right)$ at a point $L$ is the $\alpha$-space $E_{\alpha}\left(\boldsymbol{P}_{\mathbf{1}}\right) \subset \operatorname{Hom}\left(\boldsymbol{L}, \mathbb{A}^{n+1} / \boldsymbol{L}\right)$ (see Definition 2.12). Dually, the tangent space of the $\beta$-variety $\beta\left(P_{2}\right)$ at a point $L$ is the $\beta$-space $E_{\beta}\left(\boldsymbol{P}_{\mathbf{2}} / \boldsymbol{L}\right) \subset \operatorname{Hom}\left(\boldsymbol{L}, \mathbb{A}^{n+1} / \boldsymbol{L}\right)$. Conversely, given any $L \in \operatorname{Gr}\left(\ell, \mathbb{P}^{n}\right)$ and an $\alpha$-space in $T_{\operatorname{Gr}\left(\ell, \mathbb{P}^{n}\right), L}$, there is a unique $\alpha$-variety containing $L$ whose tangent space at $L$ is the given $\alpha$-space. The analogous assertion holds for $\beta$-spaces and $\beta$-varieties.

These observations show that subvarieties of $\alpha$ - and $\beta$-varieties are strongly isotropic. In fact, we can even show that all strongly isotropic varieties either are such subvarieties or have dimension one. Note that we have seen in Section 7.1 
that (strongly) isotropic curves are not necessarily contained in $\alpha$ - or $\beta$-varieties. The curve $\operatorname{Osc}_{1}(C) \subset \operatorname{Gr}\left(1, \mathbb{P}^{3}\right)$ of tangent lines to a nondegenerate irreducible curve $C \subset \mathbb{P}^{3}$ is such an example.

Theorem 7.14. 1. Every subvariety of an $\alpha$-or $\beta$-variety is strongly isotropic.

2. Every irreducible strongly isotropic variety of dimension at least two is either a subvariety of a unique $\alpha$-variety or a subvariety of a unique $\beta$-variety.

As in the classification of strongly coisotropic varieties in Section 4.3, we use Lemma 4.19 two distinguish two types of strongly isotropic varieties.

Definition/Corollary 7.15. Let $\Sigma$ be an irreducible strongly isotropic variety of dimension at least two. Either each tangent space at a smooth point of $\Sigma$ is contained in a unique $\alpha$-space, or each tangent space at a smooth point of $\Sigma$ is contained in a unique $\beta$-space. In the first case, we call $\Sigma$ strongly isotropic of $\alpha$-type. In the latter case, we say that $\Sigma$ is strongly isotropic of $\beta$-type.

Lemma 7.16. Every strongly isotropic variety in $\operatorname{Gr}\left(\ell, \mathbb{P}^{n}\right)$ of $\alpha$-type has dimension at most $n-\ell$, and every strongly isotropic variety of $\beta$-type has dimension at most $\ell+1$.

Proof. This follows from the fact that $\alpha$-spaces in $\operatorname{Hom}\left(L, \mathbb{A}^{n+1} / L\right)$ have dimension $n-\ell$ and that the dimension of $\beta$-spaces is $\ell+1$, where $L \in \operatorname{Gr}\left(\ell, \mathbb{P}^{n}\right)$.

Lemma 7.17. 1. A subvariety $\Sigma \subset \operatorname{Gr}\left(\ell, \mathbb{P}^{n}\right)$ is strongly isotropic of $\alpha$-type if and only if $\Sigma^{\perp} \subset \operatorname{Gr}\left(n-\ell-1,\left(\mathbb{P}^{n}\right)^{*}\right)$ is strongly isotropic of $\beta$-type.

2. Moreover, $\Sigma$ is contained in the $\alpha$-variety $\alpha\left(P_{1}\right)$ if and only if $\Sigma^{\perp}$ is contained in the $\beta$-variety $\beta\left(P_{1}^{\vee}\right)$.

Proof. First, we notice that $L$ is a smooth point of $\Sigma$ if and only if $L^{\vee}$ is a smooth point of $\Sigma^{\perp}$. Secondly, a linear subspace $U \subset L$ is contained in the kernel of $\varphi \in T_{\Sigma, L}$ if and only if the image of $\varphi^{*} \in T_{\Sigma^{\perp}, L^{\vee}}$ is contained in $(\boldsymbol{L} / U)^{*}$. This shows the first part of Lemma 7.17. The second part is immediate.

Thus, to prove Theorem 7.14, we only have to consider strongly isotropic varieties of $\alpha$-type. To every strongly isotropic variety $\Sigma \subset \operatorname{Gr}\left(\ell, \mathbb{P}^{n}\right)$ of $\alpha$-type, we can associate a variety $\Sigma_{\mathrm{ker}} \subset \operatorname{Gr}\left(\ell-1, \mathbb{P}^{n}\right)$ as follows. For every smooth point $L$ of $\Sigma$, there is a unique hyperplane $P_{L} \subset L$ such that every tangent vector $\varphi \in T_{\Sigma, L}$ satisfies $\boldsymbol{P}_{\mathbf{L}} \subset \operatorname{ker} \varphi$. Hence, we get a rational map

$$
\begin{aligned}
\Psi_{\text {ker }}: \Sigma & \rightarrow \operatorname{Gr}\left(\ell-1, \mathbb{P}^{n}\right), \\
\operatorname{Reg}(\Sigma) \ni L & \longmapsto P_{L},
\end{aligned}
$$

and we define $\Sigma_{\text {ker }}$ to be the Zariski closure of its image. Since $\Sigma$ is irreducible, so is $\Sigma_{\mathrm{ker}}$. We will prove Theorem 7.14 by showing that $\Sigma_{\mathrm{ker}}$ is a point.

Lemma 7.18. Let $\Sigma \subset \operatorname{Gr}\left(\ell, \mathbb{P}^{n}\right)$ be a strongly isotropic variety of $\alpha$-type. 
1. For a general point $L$ of $\Sigma$, the image of every tangent vector $\phi \in T_{\Sigma_{\mathrm{ker}}, P_{L}}$ is contained in $\mathbf{L} / \boldsymbol{P}_{\mathbf{L}}$.

2. If the general fiber of $\Psi_{\mathrm{ker}}$ contains exactly one point, then $\Sigma_{\mathrm{ker}}$ is strongly isotropic of $\beta$-type. Otherwise, $\Sigma_{\mathrm{ker}}$ must be a point.

Proof. For a general point $L \in \Sigma$, the differential

$$
D_{L} \Psi_{\mathrm{ker}}: T_{\Sigma, L} \longrightarrow T_{\Sigma_{\mathrm{ker}}, P_{L}}
$$

of $\Psi_{\text {ker }}$ at $L$ is a surjection. By Corollary 2.9 it sends a map $\varphi: L \rightarrow \mathbb{A}^{n+1} / L$ in $T_{\Sigma, L}$ to a linear map $\phi: \boldsymbol{P}_{\mathbf{L}} \rightarrow \mathbb{A}^{n+1} / \boldsymbol{P}_{\mathbf{L}}$ such that $\left.\varphi\right|_{\boldsymbol{P}_{\mathbf{L}}}=(\phi \bmod \boldsymbol{L})$. But since $\left.\varphi\right|_{\boldsymbol{P}_{\mathbf{L}}}$ is the zero-map, the image of $\phi$ must be contained in $\boldsymbol{L} / \boldsymbol{P}_{\mathbf{L}}$. In particular, the rank of $\phi$ is at most one. Since $D_{L} \Psi_{\text {ker }}$ is surjective, every $\phi \in T_{\Sigma_{\text {ker }}, P_{L}}$ has at most rank one and satisfies $\operatorname{im} \phi \subset \boldsymbol{L} / \boldsymbol{P}_{\boldsymbol{L}}$. This shows that $\Sigma_{\mathrm{ker}}$ is strongly isotropic of $\beta$-type if $\operatorname{dim} \Sigma_{\text {ker }} \geq 2$. Now we have shown the first assertion of Lemma 7.18 as well as the first part of the second assertion.

Finally, we assume that the general fiber of $\Psi_{\text {ker }}$ contains more than one point. We consider a general point $P \in \Sigma_{\text {ker }}$ and two general points $L_{1}, L_{2} \in \Psi_{\text {ker }}^{-1}(P)$ in its fiber. Both differentials $D_{L_{1}} \Psi_{\text {ker }}$ and $D_{L_{2}} \Psi_{\text {ker }}$ are surjective, and the image of every $\phi \in T_{\Sigma_{\text {ker }}, P}$ is contained in $L_{\mathbf{1}} / \boldsymbol{P} \cap \boldsymbol{L}_{\mathbf{2}} / \boldsymbol{P}=\{0\}$. Thus, the dimension of $\Sigma_{\text {ker }}$ must be zero.

Proof of Theorem 7.14 The first part follows immediately from Remark 7.13. For the second part, let $\Sigma \subset \operatorname{Gr}\left(\ell, \mathbb{P}^{n}\right)$ be an irreducible strongly isotropic variety of dimension at least two. Due to Lemma 7.17, we can assume that $\Sigma$ is of $\alpha$ type. Thus, we have a dominant rational map $\Psi_{\text {ker }}: \Sigma \rightarrow \Sigma_{\text {ker }}$ as in (7.2). Moreover, we assume for contradiction that $\Sigma_{\mathrm{ker}} \subset \operatorname{Gr}\left(\ell-1, \mathbb{P}^{n}\right)$ is not a point. By Lemma 7.18, the general fiber of $\Psi_{\text {ker }}$ consists of exactly one point and $\Sigma_{\text {ker }}$ is strongly isotropic of $\beta$-type. In particular, $\operatorname{dim} \Sigma_{\mathrm{ker}}=\operatorname{dim} \Sigma$.

We denote by $X_{\mathrm{ker}} \subset \mathbb{P}^{n}$ the variety swept out by all $P \in \Sigma_{\mathrm{ker}}$. It is the image of the incidence correspondence $F_{\text {ker }}:=\left\{(x, P) \in \mathbb{P}^{n} \times \Sigma_{\text {ker }} \mid x \in P\right\}$ under the projection $\pi$ onto the first factor; so $\operatorname{dim} X_{\text {ker }} \leq \operatorname{dim} F_{\text {ker }}=\operatorname{dim} \Sigma+\ell-1 \leq$ $n-1$ by Lemma7.16. At a smooth point $(x, P) \in F_{\text {ker }}$ such that $P \in \operatorname{Reg}\left(\Sigma_{\text {ker }}\right)$ the incidence correspondence has the tangent space

$$
T_{F_{\mathrm{ker},}(x, P)}=\left\{(\phi, \varphi) \in \operatorname{Hom}\left(\boldsymbol{x}, \mathbb{A}^{n+1} / \boldsymbol{x}\right) \times T_{\Sigma_{\mathrm{ker}, P}}|\varphi|_{\boldsymbol{x}}=(\phi \bmod \boldsymbol{P})\right\},
$$

since the inclusion " $C$ " follows from Lemma 2.7 and both linear spaces in (7.3) have the same dimension.

Let $(x, P) \in F_{\text {ker }}$ be general. As the differential $D_{(x, P)} \pi: T_{F_{\mathrm{ker}},(x, P)} \rightarrow T_{X_{\mathrm{ker},},}$ of $\pi$ at $(x, P)$ is surjective, we see from (7.3) that

$$
T_{X_{\mathrm{ke},}, x}=\left\{\phi \in \operatorname{Hom}\left(\boldsymbol{x}, \mathbb{A}^{n+1} / \boldsymbol{x}\right)\left|\exists \varphi \in T_{\Sigma_{\mathrm{ke},} P}: \varphi\right|_{\boldsymbol{x}}=(\phi \bmod \boldsymbol{P})\right\} .
$$

We denote by $L \in \Sigma$ the unique point in the fiber $\Psi_{\text {ker }}^{-1}(P)$. By Lemma 7.18 , the 
space $T_{X_{\mathrm{ker}}, x}$ is a subset of $\mathrm{H}_{x, L}:=\left\{\phi \in \operatorname{Hom}\left(\boldsymbol{x}, \mathbb{A}^{n+1} / \boldsymbol{x}\right) \mid \operatorname{im} \phi \subset \boldsymbol{L} / \boldsymbol{x}\right\}$. The dimension of $\mathrm{H}_{x, L}$ is $\ell$, which implies that the dimension of $X_{\text {ker }}$ is at most $\ell$. Since we assumed that $\Sigma_{\mathrm{ker}} \subset \operatorname{Gr}\left(\ell-1, \mathbb{P}^{n}\right)$ is not a point, the dimension of $X_{\text {ker }}$ must be exactly $\ell$, so $T_{X_{\mathrm{ker}}, x}=\mathrm{H}_{x, L}$. Moreover, the dimension of the fiber $\pi^{-1}(x)$ is $\operatorname{dim} F_{\text {ker }}-\operatorname{dim} X_{\text {ker }}=\operatorname{dim} \Sigma-1 \geq 1$. We pick another general point $\left(x, P^{\prime}\right)$ in this fiber and repeat the above argument. We see that the unique point $L^{\prime} \in \Sigma$ in the fiber $\Psi_{\mathrm{ker}}^{-1}\left(P^{\prime}\right)$ also satisfies $T_{X_{\mathrm{ker},}}=\mathrm{H}_{x, L^{\prime}}$. In particular, we have $\mathrm{H}_{x, L}=\mathrm{H}_{x, L^{\prime}}$, so $L=L^{\prime}$ and $P=P^{\prime}$, but this is a contradiction since $(x, P)$ and $\left(x, P^{\prime}\right)$ were generally chosen on the variety $\pi^{-1}(x)$ of dimension at least one.

Thus, we have shown that $\Sigma_{\text {ker }}$ must be a point, i.e., an $(\ell-1)$-dimensional subspace $P \subset \mathbb{P}^{n}$. Every $L \in \Sigma$ contains $P$, so $\Sigma \subset \alpha(P)$.

\subsection{Congruences}

So far we have classified all subvarieties of $\operatorname{Gr}\left(1, \mathbb{P}^{3}\right)$ of dimension one or three which are isotropic and coisotropic at the same time by their underlying projective varieties. Coisotropic hypersurfaces, which are trivially isotropic by Lemma 7.3, have been studied in Chapter 3, and isotropic curves, which are trivially coisotropic by Corollary 4.5, have been discussed in Section 7.1. Now we focus on congruences, as these are also isotropic and coisotropic.

Congruences can arise naturally from subvarieties of $\mathbb{P}^{3}$, as we have seen in Chapter 5. In the following, we show that every congruence is associated to a projective variety, as we summarize in Table 7.2. The key notion for this classification is the (strict) focal locus of a congruence. Recall that the order $\alpha$ of a congruence $\Sigma$ is the number of lines on $\Sigma$ which pass through a general point $p \in \mathbb{P}^{3}$. A point $p \in \mathbb{P}^{3}$ is a focal point of $\Sigma$ if the number of lines on $\Sigma$ passing though $p$ differs from the order $\alpha$. In other words, the focal locus of $\Sigma$ is the branch locus of the projection

$$
\left\{(p, L) \in \mathbb{P}^{3} \times \Sigma \mid p \in L\right\} \longrightarrow \mathbb{P}^{3}
$$

onto the first factor. For example, by Theorems 6.5 and 6.18, the visual event surface of a general curve or surface $X \subset \mathbb{P}^{3}$ is the focal locus of the singular locus of the Chow or Hurwitz hypersurface of $X$, respectively.

Lemma 7.19. Let $\Sigma \subset \operatorname{Gr}\left(1, \mathbb{P}^{3}\right)$ be a congruence. A point $p \in \mathbb{P}^{3}$ is a focal point of $\Sigma$ if and only if one of the following two conditions is satisfied:

1. $p$ is contained in some line $L \in \operatorname{Sing}(\Sigma)$, or

2. $p$ is contained in some line $L \in \operatorname{Reg}(\Sigma)$ such that there is a non-zero $\varphi \in T_{\Sigma, L}$ with $\boldsymbol{p} \subset \operatorname{ker} \varphi$.

Proof. As in the proofs of Theorems 6.5 and 6.18, we see that $p$ is not a focal point if and only if the $\alpha$-variety $\alpha(p)$ of lines passing through $p$ intersects the 
congruence $\Sigma$ at exactly $\alpha$ (here denoting the order of $\Sigma$ ) points. This is equivalent to that $\alpha(p)$ intersects $\Sigma$ only at smooth points and the intersection at all those points is transverse (cf. [34, Thm. 1.26]). Since the tangent space of the $\alpha$ variety $\alpha(p)$ at a point $L$ is the $\alpha$-space $E_{\alpha}(\boldsymbol{p}) \subset \operatorname{Hom}\left(\boldsymbol{L}, \mathbb{A}^{4} / \boldsymbol{L}\right)$, we have shown Lemma 7.19 .

Remark 7.20. By Corollary 2.11, the second condition in Lemma 7.19 is equivalent to that the embedded tangent plane of the image of $\Sigma$ under the Plücker embedding $\mathrm{pl}$ at the point $\mathrm{pl}(L)$ meets the embedded $\alpha$-plane $\operatorname{pl}(\alpha(p))$ in at least a line of $\mathbb{P}^{5}$ (i.e., a pencil of lines in $\mathbb{P}^{3}$ ). This is in fact the definition of $p$ being a focal point given by Goldstein in [44]. For smooth congruences, this definition agrees with the definition given above.

A line $L \in \Sigma$ is called a focal line if every point on $L$ is a focal point. In particular, every $L$ in the singular locus of $\Sigma$ is a focal line. The strict focal locus of $\Sigma$ is the Zariski closure of the branch locus of the projection

$$
\begin{array}{r}
\left\{(p, L) \in \mathbb{P}^{3} \times \Sigma \mid p \in L, L \text { is not focal }\right\} \\
(p, L) \longmapsto \mathbb{P}^{3},
\end{array}
$$

Example 7.21. By Theorems 6.5 and 5.8, the focal locus of the secant congruence of a general curve $C \subset \mathbb{P}^{3}$ is the visual event surface of $C$. We will see in Proposition 7.35 that the strict focal locus of the secant congruence is only $C$. $\diamond$

Example 7.22. Every line on an $\alpha$-variety $\alpha(p)$ has exactly one focal point, namely the point $p$ itself. In particular, $\alpha(p)$ has no focal lines, and both its focal locus and strict focal locus contain only the point $p$.

Dually, we consider the $\beta$-variety $\beta(H)$ of lines contained in a plane $H$. Every line on this congruence is a focal line. Its focal locus is the plane $H$, but its strict focal locus is empty.

Remark 7.23. Recall that the class $\beta$ of a congruence $\Sigma$ is the number of lines on $\Sigma$ which lie in a general plane. A plane $H \subset \mathbb{P}^{3}$ is a focal plane of $\Sigma$ if the number of lines on $\Sigma$ lying in $H$ differs from the class $\beta$. Equivalently, a focal plane $H \subset \mathbb{P}^{3}$ of $\Sigma \subset \operatorname{Gr}\left(1, \mathbb{P}^{3}\right)$ corresponds to a focal point $H^{\vee}$ of $\Sigma^{\perp} \subset \operatorname{Gr}\left(1,\left(\mathbb{P}^{3}\right)^{*}\right)$.

Analogously to the strict focal locus, one may define the strict locus of focal planes, which consists of those planes that are projectively dual to points in the strict focal locus of $\Sigma^{\perp}$.

Congruences have first been classified according to their (strict) focal loci by Kummer [69], who studied only those of order one. General classifications have been classically known, but usually not described exhaustively. For example, the characterization of congruences on page 417 of [94] misses the cases denoted by "Seg-type 1" in Table 7.2. Many more classical and modern sources study focal loci of congruences, like [55, Ch. XIV] or [95, 92, 44, 5]. We give a complete classification of congruences in Table 7.2.

By Theorem 7.14, every irreducible strongly isotropic (and thus strongly coisotropic) congruence must be either an $\alpha$ - or a $\beta$-variety. Hence, we focus here 


\begin{tabular}{|c|c|c|}
\hline notation & description & Seg-type \\
\hline $\begin{array}{l}\alpha(x) \\
\beta(H)\end{array}$ & $\begin{array}{l}\text { lines through a fixed point } x \\
\text { lines in a fixed plane } H\end{array}$ & $\infty$ \\
\hline $\begin{array}{l}\text { interse } \\
\mathrm{CH}_{0}\left(C_{1}\right) \cap \mathrm{CH}_{0}\left(C_{2}\right) \\
\mathrm{CH}_{0}(C) \cap \mathrm{CH}_{1}(S) \\
\mathrm{CH}_{1}\left(S_{1}\right) \cap \mathrm{CH}_{1}\left(S_{2}\right)\end{array}$ & $\begin{array}{l}\text { tion of two coisotropic hypersurfaces } \\
\text { lines intersecting two given curves } \\
\text { lines meeting a curve and tangent to a surface } \\
\text { lines tangent to two given surfaces }\end{array}$ & 2 \\
\hline $\begin{array}{l}\operatorname{Sec}(C) \\
\operatorname{Bit}(S)\end{array}$ & $\begin{array}{l}\text { rsection of a coisotropic hypersurface } \\
\text { secant lines to a given curve } \\
\text { bitangent lines to a given surface }\end{array}$ & 2 \\
\hline $\begin{array}{l}\operatorname{Infl}(S) \\
\Sigma(\mathcal{C})\end{array}$ & $\begin{array}{l}\text { inflectional tangent lines to a given surface } \\
\sum(\mathcal{C}) \text { is the image of } \quad(x, L, y) \mapsto L \\
\left\{(x, L, y) \mid(x, y) \in \mathcal{C}, x \in L \subset y^{\vee}\right\} \rightarrow \operatorname{Gr}\left(1, \mathbb{P}^{3}\right) \text { for } \\
\text { curves } X \subset \mathbb{P}^{3}, Y \subset X^{\vee}, \mathcal{C} \subset \mathcal{N}_{X, X^{\vee}} \cap(X \times Y)\end{array}$ & 1 \\
\hline
\end{tabular}

Table 7.2: All types of congruences in $\operatorname{Gr}\left(1, \mathbb{P}^{3}\right)$.

The value in the column Seg-type denotes $\left|\mathbb{P}\left(T_{\Sigma, L}\right) \cap \operatorname{Seg}\left(\boldsymbol{L}, \mathbb{A}^{4} / \boldsymbol{L}\right)\right|$ for a general $L$ in the congruence $\Sigma$.

on irreducible congruences which are not strongly isotropic. For a general point $L$ of such a congruence $\Sigma$, the projective line $\mathbb{P}\left(T_{\Sigma, L}\right)$ intersects the corresponding Segre variety $\operatorname{Seg}\left(L, \mathbb{A}^{4} / L\right) \cong \mathbb{P}^{1} \times \mathbb{P}^{1}$ either at one or at two points. In other words (by Lemma 7.19), a general $L \in \Sigma$ contains either one or two focal points. We call this number the Seg-type of the congruence. Note that the congruences $\Sigma$ and $\Sigma^{\perp}$ have the same Seg-type by Remark/Definition 2.6 .

All congruences listed in Table 7.2 appeared before in this thesis, except the congruences in the last row. Here $\mathcal{N}_{X, X^{\vee}} \subset X \times X^{\vee}$ denotes the conormal variety of an irreducible curve $X \subset \mathbb{P}^{3}$, and $\mathcal{C} \subset \mathcal{N}_{X, X^{\vee}}$ is an irreducible curve whose projections onto the first and second factor surject onto $X$ and a curve $Y \subset X^{\vee}$. The congruence $\Sigma(\mathcal{C})$ is defined as the image of the incidence correspondence $\left\{(x, L, y) \in X \times \operatorname{Gr}\left(1, \mathbb{P}^{3}\right) \times Y \mid(x, y) \in \mathcal{C}, x \in L \subset y^{\vee}\right\}$ under the projection onto the middle factor. Since $X$ and $Y$ are curves, they are projectively dual to each other if and only if they are both lines.

Example 7.24. Let $X \subset \mathbb{P}^{3}$ be an irreducible nondegenerate curve and let $Y=$ $\mathrm{Osc}_{2}(X)^{\perp} \subset\left(\mathbb{P}^{3}\right)^{*}$ be its osculating dual curve. In this situation, $\mathcal{C}$ is the Zariski closure of the set of all $(x, y)$ where $y^{\vee}$ is the osculating plane at $x \in \operatorname{Reg}(X)$. Hence, $\Sigma(\mathcal{C})$ consists of the lines in osculating planes of $X$ passing through the corresponding point of osculation.

Remark 7.25. Let us compare the classification of all congruences in Table 7.2 with Kummer's classification of order one congruences. The following version of Kummer's result using modern mathematical language was derived by De Poi in [29]. A congruence $\Sigma$ of bidegree $(1, \beta)$ is either

1. $\alpha(x)$ for some $x \in \mathbb{P}^{3}$ (here $\beta=0$ ), or 
2. the secant congruence of a twisted cubic (here $\beta=3$ ),

3. the family of lines meeting both a rational curve $C$ of degree $\beta$ and a line $\mathcal{L}$, where $\mathcal{L}$ and $C$ intersect in $\beta-1$ points (counted with multiplicities), or

4. the following special case of the last row of Table 7.2 for a line $\mathcal{L} \subset \mathbb{P}^{3}$ and a non-constant morphism $\Phi: \mathcal{L}^{\vee} \rightarrow \mathcal{L}$ of degree $\beta$, the congruence $\Sigma$ is the union of all pencils of lines $\left\{L \in \operatorname{Gr}\left(1, \mathbb{P}^{3}\right) \mid \Phi(y) \in L \subset y^{\vee}\right\}$ for $y \in \mathcal{L}^{\vee}$.

Congruences of Seg-type one. For a congruence $\Sigma$ of Seg-type one, we denote by $\Sigma^{\circ} \subset \Sigma$ the subset of all $L \in \operatorname{Reg}(\Sigma)$ with $\left|\mathbb{P}\left(T_{\Sigma, L}\right) \cap \operatorname{Seg}\left(\boldsymbol{L}, \mathbb{A}^{4} / \boldsymbol{L}\right)\right|=1$. By Lemma 7.19, all lines $L \in \Sigma^{\circ}$ contain exactly one focal point and are contained in exactly one focal plane. We define the incidence correspondence

$$
\begin{aligned}
F_{\Sigma} & :=\left\{(x, L, H) \mid L \in \Sigma^{\circ}, \exists \varphi \in T_{\Sigma, L}: \operatorname{ker} \varphi=\boldsymbol{x}, \operatorname{im} \varphi=\boldsymbol{H} / \boldsymbol{L}\right\} \\
& \subset \mathbb{P}^{3} \times \operatorname{Gr}\left(1, \mathbb{P}^{3}\right) \times \operatorname{Gr}\left(2, \mathbb{P}^{3}\right) .
\end{aligned}
$$

Identifying $\left(\mathbb{P}^{3}\right)^{*}$ with $\operatorname{Gr}\left(2, \mathbb{P}^{3}\right)$, we get three projections $\pi_{1}: F_{\Sigma} \rightarrow \mathbb{P}^{3}$, $\pi: F_{\Sigma} \rightarrow \Sigma$, and $\pi_{2}: F_{\Sigma} \rightarrow\left(\mathbb{P}^{3}\right)^{*}$. We denote by $X:=X(\Sigma):=\overline{\operatorname{im}\left(\pi_{1}\right)} \subset \mathbb{P}^{3}$ and $Y:=Y(\Sigma):=\overline{\operatorname{im}\left(\pi_{2}\right)} \subset\left(\mathbb{P}^{3}\right)^{*}$ the respective images. Note that $X$ is the strict focal locus of $\Sigma$ and that $Y$ is the strict focal locus of $\Sigma^{\perp}$. Since the map $\pi$ is finite of degree one and we assume $\Sigma$ to be irreducible, $X$ and $Y$ are irreducible varieties. Since $\Sigma$ has dimension two, the dimensions of $X$ and $Y$ are at most two.

First, we observe that neither $X$ nor $Y$ can be a point. Indeed, if $X$ would be a point $x$, then every line on $\Sigma$ would need to pass through $x$, and thus $\Sigma=\alpha(x)$. Similarly, if $Y$ would be a point $y$, then $\Sigma$ would need to be $\beta\left(y^{\vee}\right)$. In any of these two cases, every tangent vector of $\Sigma$ would be a homomorphism of rank at most one.

Proposition 7.26. Let $\Sigma$ be an irreducible congruence of Seg-type one. For a general $L \in \Sigma$ with $(x, L, H) \in F_{\Sigma}$, we have that

$$
\mathbb{T}_{X, x} \subset H \quad \text { and } \quad \mathbb{T}_{Y, H^{\vee}} \subset x^{\vee} .
$$

Proof. $\mathbb{P}\left(T_{\Sigma, L}\right)$ is a tangent line to the quadric $\operatorname{Seg}\left(\boldsymbol{L}, \mathbb{A}^{4} / \boldsymbol{L}\right) \cong \mathbb{P}^{1} \times \mathbb{P}^{1}$ at the projective point corresponding to $\varphi \in T_{\Sigma, L}$ with $\operatorname{ker} \varphi=\boldsymbol{x}$ and $\operatorname{im} \varphi=\boldsymbol{H} / \boldsymbol{L}$. Thus, it is contained in the tangent plane to the Segre variety at this point, which consist of all $\varphi: \boldsymbol{L} \rightarrow \mathbb{A}^{4} / \boldsymbol{L}$ with $\varphi(\boldsymbol{x}) \subset \boldsymbol{H} / \boldsymbol{L}$. In particular, every map $\varphi \in T_{\Sigma, L}$ satisfies $\varphi(\boldsymbol{x}) \subset \boldsymbol{H} / \boldsymbol{L}$. Since $L \in \Sigma$ is general, the differential of $\pi$ at $(x, L, H)$ is an isomorphism $T_{F_{\Sigma},(x, L, H)} \rightarrow T_{\Sigma, L}$. Furthermore, the differential of $\pi_{1}$ at $(x, L, H)$ surjects $T_{F_{\Sigma},(x, L, H)}$ onto $T_{X, x}$; analogously for $\pi_{2}$. Thus, we get surjections $\Phi: T_{\Sigma, L} \rightarrow T_{X, x}$ and $\Psi: T_{\Sigma, L} \rightarrow T_{Y, H^{\vee}}$ as in the following commutative diagram: 


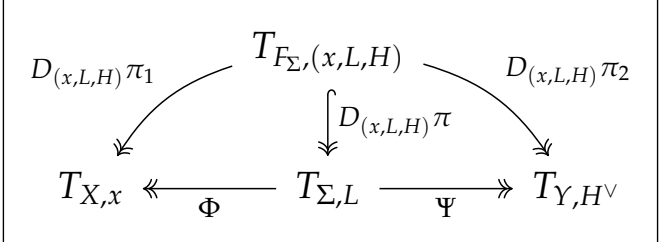

By Lemma 2.7. we have $\left.\varphi\right|_{\boldsymbol{x}}=(\Phi(\varphi) \bmod \boldsymbol{L})$ and $\left.\Psi(\varphi)^{*}\right|_{\boldsymbol{L}}=(\varphi \bmod \boldsymbol{H})$ for all $\varphi \in T_{\Sigma, L}$. Hence, for every $\varphi \in T_{\Sigma, L}$, the image of $\Phi(\varphi): \boldsymbol{x} \rightarrow \mathbb{A}^{4} / \boldsymbol{x}$ is contained in $\boldsymbol{H} / \boldsymbol{x}$, and the kernel of $\Psi(\varphi)^{*}: \boldsymbol{H} \rightarrow \mathbb{A}^{4} / \boldsymbol{H}$ contains $\boldsymbol{x}$.

An immediate corollary from Proposition 7.26 is that $Y \subset X^{\vee}$ and $X \subset Y^{\vee}$. Moreover, in the situation of Proposition 7.26, if $X$ is a surface, then $L$ is a tangent line to $X$ at $x$. Dually, if $Y$ is a surface, then $L^{\vee}$ is a tangent line to $Y$ at $H^{\vee}$.

Proposition 7.27. Let $\Sigma$ be an irreducible congruence of Seg-type one. If its strict focal locus $X$ is a curve, then $Y$ is a curve and $\Sigma=\Sigma(\mathcal{C})$ for some curve $\mathcal{C} \subset \mathcal{N}_{X, X \vee}$ whose projections onto the first and second factor surject onto $X$ and $Y$, respectively.

Proof. First we assume for contradiction that $Y$ is a surface. Our idea is to foliate $\Sigma$ into a one-dimensional family of isotropic curves. We do this by following the rank one direction at each $L \in \Sigma^{\circ}$. To be more precise, we consider a general $L \in \Sigma$ with $(x, L, H) \in F_{\Sigma}$. Locally around $L$, there is an analytic curve $\Gamma \subset \Sigma$ through $L$ which is isotropic. As in Section 7.1, we get an analytic curve $\Gamma^{+} \subset$ $Y \subset\left(\mathbb{P}^{3}\right)^{*}$ around $H^{\vee}$ such that $L^{\vee}$ is the tangent line to $\Gamma^{+}$at $H^{\vee}$. Similarly, all points in $\Gamma$ correspond to tangent lines of $\Gamma^{+}$. All such curves $\Gamma^{+}$that arise from the isotropic curves $\Gamma$ in the foliation of $\Sigma$ form a foliation of $Y$. Note that $\Gamma^{+}$cannot be a point since $\operatorname{dim} Y=2$ and $L$ was chosen generally. On the other hand, $\Gamma^{-} \subset X$ cannot be a curve, because otherwise every $L \in \Sigma$ would need to be a tangent line to the curve $X$. So $\Gamma^{-}$is the point $x \in X$, which implies that $\Gamma^{+}$ is contained in the plane $x^{\vee}$. By Proposition 7.26, we have that $Y=X^{\vee}$ and that $x^{\vee}=\mathbb{T}_{Y, y}$ for general $y \in \Gamma^{+}$. Since $L \in \Sigma$ was chosen generally, $x$ is a general point of $X$ and $x^{\vee}$ is the tangent plane to $Y$ along a line. In particular, $\Gamma^{+}$is a line, but this implies that $\Gamma=\{L\}$, which contradicts that $\operatorname{dim} \Gamma=1$.

Hence, $Y$ is a curve. As above, we consider an analytic isotropic curve $\Gamma \subset \Sigma$ through a general $L \in \Sigma$ with $(x, L, H) \in F_{\Sigma}$. In this situation, $\Gamma^{-} \subset X$ is the point $x \in X$ and $\Gamma^{+} \subset Y$ is the point $H^{\vee} \in Y$. Thus, every line in $\Gamma$ passes through $x$ and lies in the plane $H$. In particular, the whole pencil of lines in $H$ passing through $x$ must be contained in $\Sigma$, and we have $\left(x, L^{\prime}, H\right) \in F_{\Sigma}$ for a general line $L^{\prime}$ in this pencil. By Proposition 7.26, we have $\left(x, H^{\vee}\right) \in \mathcal{N}_{X, X^{\vee}}$. As the above argument holds for general $L \in \Sigma$, the image of $\pi_{1} \times \pi_{2}: F_{\Sigma} \rightarrow X \times Y$ is a curve $\mathcal{C}$ satisfying Proposition 7.27.

Proposition 7.28. Let $\Sigma$ be an irreducible congruence of Seg-type one. If its strict focal locus $X$ is a surface, then $Y$ is a surface and $\Sigma$ is the inflectional congruence of $X$.

Proof. By Proposition 7.27, the variety $Y$ is a surface, which is actually dual to $X$ by Proposition 7.26. We consider a general point $L \in \Sigma$ and $(p, L, H) \in F_{\Sigma}$. Locally around $L$, there is an analytic curve $\Gamma \subset \Sigma$ through $L$ which is isotropic. 
As in Section 7.1 (and in the proof of Proposition 7.27), we get analytic curves $\Gamma^{-} \subset X$ and $\Gamma^{+} \subset Y$ around $p$ and $H^{\vee}$, respectively, such that $L$ is the tangent line to $\Gamma^{-}$at $p$ and $H$ is the osculating plane of $\Gamma^{-}$at $p$ (and vice versa). By Proposition 7.26, the plane $H$ is also the embedded tangent plane of $X$ at $p$. From this, we will show that $L$ is an inflectional line of $X$ at $p$.

We choose coordinates such that $p=(1: 0: 0: 0)$ and work in the affine chart $\mathbb{P}^{3} \backslash Z\left(x_{0}\right)$. We parametrize $\Gamma^{-}$by a local analytic isomorphism $\gamma$ from a neighborhood of the origin in $\mathbb{A}^{1}$ to a neighborhood of the point $p$ in $\Gamma^{-}$. This map has the form $\gamma(t)=\left(\gamma_{1}(t), \gamma_{2}(t), \gamma_{3}(t)\right)$ for some $\gamma_{1}, \gamma_{2}, \gamma_{3} \in \mathbb{C} \llbracket t \rrbracket$ with $\gamma(0)=(0,0,0)$. Furthermore, we denote by $f=f_{1}+f_{2}+\ldots \in \mathbb{C}\left[x_{1}, x_{2}, x_{3}\right]$ a defining polynomial for $X$ in our affine chart, where $f_{i}$ is homogeneous of degree $i$. Since we already know that $L$ is a tangent line to $X$ at $p$, we only have to show that $f_{2}\left(\gamma^{\prime}(0)\right)=0$.

For all $t$ in the neighborhood around the origin in $\mathbb{A}^{1}$, we have $f(\gamma(t))=0$. In particular,

$$
0=\frac{\partial^{2}(f \circ \gamma)}{\partial^{2} t}(0)=2 f_{2}\left(\gamma^{\prime}(0)\right)+f_{1}\left(\gamma^{\prime \prime}(0)\right)
$$

Since $H$ is the osculating plane of $\Gamma^{-}$at $p$, it contains the point $\gamma^{\prime \prime}(0)$. Moreover, since $H$ is also $\mathbb{T}_{X, p}$, this point is in the zero locus of $f_{1}$. Together with $(7.5)$, this shows $f_{2}\left(\gamma^{\prime}(0)\right)=0$.

Finally, we prove the converse of Propositions 7.27 and 7.28.

Proposition 7.29. For irreducible curves $X \subset \mathbb{P}^{3}, Y \subset X^{\vee}$ and $\mathcal{C} \subset \mathcal{N}_{X, X \vee}$ such that the projections of $\mathcal{C}$ onto the first and second factor surject onto $X$ and $Y$, respectively, the congruence $\Sigma(\mathcal{C})$ is of Seg-type one, $X(\Sigma(\mathcal{C}))=X$ and $Y(\Sigma(\mathcal{C}))=Y$.

Proof. For $L \in \Sigma:=\Sigma(\mathcal{C})$ general, there is $\left(x, H^{\vee}\right) \in \mathcal{C}$ with $x \in L \subset H$ such that $\Gamma_{L}:=\left\{L^{\prime} \in \operatorname{Gr}\left(1, \mathbb{P}^{3}\right) \mid x \in L^{\prime} \subset H\right\} \subset \Sigma$. Hence,

$$
T_{\Sigma, L} \supset T_{\Gamma_{L}, L}=\left\{\varphi \in \operatorname{Hom}\left(\boldsymbol{L}, \mathbb{A}^{4} / \boldsymbol{L}\right) \mid \boldsymbol{x} \subset \operatorname{ker} \varphi, \operatorname{im} \varphi \subset \boldsymbol{H} / \boldsymbol{L}\right\} .
$$

Since $\Sigma$ is contained in the Chow hypersurface of $X$, we see from (3.3) that every $\varphi \in T_{\Sigma, L}$ satisfies $\varphi(\boldsymbol{x}) \subset\left(\mathbb{T}_{\boldsymbol{X}, \boldsymbol{x}}+\boldsymbol{L}\right) / \boldsymbol{L}=\boldsymbol{H} / \boldsymbol{L}$. So $\mathbb{P}\left(T_{\Sigma, L}\right)$ is a tangent line of $\operatorname{Seg}\left(\boldsymbol{L}, \mathbb{A}^{4} / \boldsymbol{L}\right)$ at the point $\mathbb{P}\left(T_{\Gamma_{L}, L}\right)$.

Since $L$ was chosen generally, we have shown Proposition 7.29 .

Proposition 7.30. Let $S \subset \mathbb{P}^{3}$ be an irreducible surface with a dual surface $S^{\vee}$. If $\operatorname{dim} \operatorname{Infl}(S)=2$, the congruence $\operatorname{Infl}(S)$ is of Seg-type one and $X(\operatorname{Infl}(S))=S=$ $Y(\operatorname{Infl}(S))^{\vee}$.

Proof. We have proven this statement already for general surfaces $S \subset \mathbb{P}^{3}$ in Theorems 4.26 and 5.45. Here, we give a different proof without using local calculations.

We define $\Sigma:=\operatorname{Infl}(S)$ and

$$
F_{S}:=\overline{\left\{\left(x, L, H^{\vee}\right) \mid x \in \operatorname{Reg}(S), H=\mathbb{T}_{S, x}, L \text { is an inflectional line to } S \text { at } x\right\}}
$$




$$
\subset S \times \Sigma \times S^{\vee}
$$

For a general $L \in \Sigma$, there is exactly one point $x \in S$ such that $L$ is an inflectional line to $S$ at $x$. We set $H:=\mathbb{T}_{S, x}$. Moreover, the differentials of the canonical projections $F_{S} \rightarrow S, F_{S} \rightarrow S^{\vee}$ and $F_{S} \rightarrow \Sigma$ yield isomorphisms from $T_{F_{S},\left(x, L, H^{\vee}\right)}$ to $T_{S, x}, T_{S^{\vee}, H^{\vee}}$ and $T_{\Sigma, L}$. Composing these yields isomorphisms $\Phi: T_{S, x} \rightarrow T_{\Sigma, L}$ and $\Psi: T_{S^{\vee}, H^{\vee}} \rightarrow T_{\Sigma, L}$ as in the following commutative diagram:

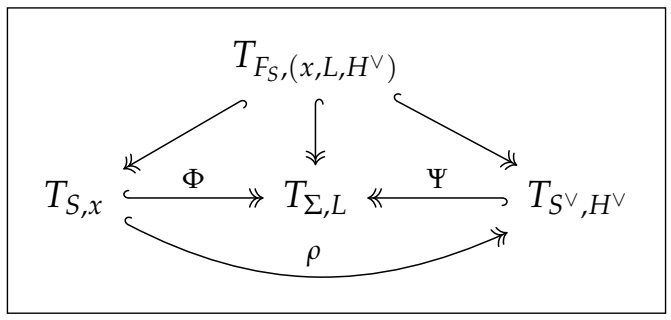

By Lemma 2.7, we have

$$
\begin{aligned}
& \left.\Phi(\phi)\right|_{\boldsymbol{x}}=(\phi \quad \bmod \boldsymbol{L}) \quad \text { for all } \phi \in T_{S, x} \text { and } \\
& \left.\psi^{*}\right|_{\boldsymbol{L}}=(\Psi(\psi) \quad \bmod \boldsymbol{H}) \quad \text { for all } \psi \in T_{S^{\vee}, H^{\vee}} \text {. }
\end{aligned}
$$

Besides, the isomorphism $\rho: T_{S, x} \rightarrow T_{S^{\vee}, H^{\vee}}$ which arises as the differential of the Gauss map $S \rightarrow S^{\vee}$ satisfies $\Phi=\Psi \circ \rho$. As $L$ is in particular a tangent line to $S$ at $x$, there is $\phi_{1} \in T_{S, x}$ with $\operatorname{im} \phi_{1}=\boldsymbol{L} / \boldsymbol{x}$. By (7.6), the kernel of $\varphi_{1}:=\Phi\left(\phi_{1}\right)$ is $\boldsymbol{x}$. Since the kernel of $\rho\left(\phi_{1}\right)^{*}$ is $\boldsymbol{L},(7.6)$ implies $\operatorname{im} \varphi_{1}=\operatorname{im} \Psi\left(\rho\left(\phi_{1}\right)\right)=\boldsymbol{H} / \boldsymbol{L}$. To show that the line $\mathbb{P}\left(T_{\Sigma, L}\right)$ is tangent to $\operatorname{Seg}\left(\boldsymbol{L}, \mathbb{A}^{4} / \boldsymbol{L}\right)$ at the projective point corresponding to $\varphi_{1}$, we only have to show that $\mathbb{P}\left(T_{\Sigma, L}\right)$ is contained in the tangent plane of the Segre variety at this point. This means that every $\varphi \in T_{\Sigma, L}$ needs to satisfy $\varphi(\boldsymbol{x}) \subset \boldsymbol{H} / \boldsymbol{L}$. But this follows from $(7.6)$ and the fact that the image of every $\phi \in T_{S, x}$ is contained in $\boldsymbol{H} / \boldsymbol{x}$.

Since $L$ was chosen generally, we have shown Proposition 7.30

Corollary 7.31. Let $S \subset \mathbb{P}^{3}$ be an irreducible surface with a dual surface $S^{\vee}$. If its inflectional locus is a congruence, we have that $\operatorname{Infl}(S)^{\perp}=\operatorname{Infl}\left(S^{\vee}\right)$.

Proof. We know that the congruence $\operatorname{Infl}(S)^{\perp} \subset \operatorname{Gr}\left(1,\left(\mathbb{P}^{3}\right)^{*}\right)$ is of Seg-type one and that $X\left(\operatorname{Infl}(S)^{\perp}\right)=Y(\operatorname{Infl}(S))$. By Proposition 7.30, we have $X\left(\operatorname{Infl}(S)^{\perp}\right)=$ $S^{\vee}$. Thus, Proposition 7.28 implies that $\operatorname{Infl}(S)^{\perp}=\operatorname{Infl}\left(S^{\vee}\right)$.

Remark 7.32. If the surface $S \subset \mathbb{P}^{3}$ is the dual of a curve, the inflectional locus of $S$ consists of a curve and possibly several $\beta$-varieties; see Proposition 5.19 and Remark 5.23 .

Congruences of Seg-type two. For a congruence $\Sigma$ of Seg-type two, we denote by $\Sigma^{\circ} \subset \Sigma$ the subset of all $L \in \operatorname{Reg}(\Sigma)$ such that $\left|\mathbb{P}\left(T_{\Sigma, L}\right) \cap \operatorname{Seg}\left(\boldsymbol{L}, \mathbb{A}^{4} / \boldsymbol{L}\right)\right|=2$. By Lemma 7.19, all lines $L \in \Sigma^{\circ}$ contain exactly two focal points and are contained in exactly two focal planes. We define the incidence correspondence $F_{\Sigma}$ as in (7.4). We consider again the projections $\pi_{1}: F_{\Sigma} \rightarrow \mathbb{P}^{3}, \pi: F_{\Sigma} \rightarrow \Sigma$, 
and $\pi_{2}: F_{\Sigma} \rightarrow\left(\mathbb{P}^{3}\right)^{*}$, with their images $X:=X(\Sigma):=\overline{\operatorname{im}\left(\pi_{1}\right)} \subset \mathbb{P}^{3}$ and $Y:=Y(\Sigma):=\overline{\operatorname{im}\left(\pi_{2}\right)} \subset\left(\mathbb{P}^{3}\right)^{*}$. Note again that $X$ is the strict focal locus of $\Sigma$ and that $Y$ is the strict focal locus of $\Sigma^{\perp}$. In this situation, the map $\pi$ is finite of degree two, and since we assume $\Sigma$ to be irreducible, as schemes $X$ and $Y$ have exactly two irreducible reduced components each. We denote these components by $X_{1}, X_{2}$ and $Y_{1}, Y_{2}$, respectively. As before, the dimension of each of those components is either one or two. During the rest of this section, we will see that the components of $Y$ are in fact projectively dual to the components of $X$, which is generally not the case for congruences $\Sigma(\mathcal{C})$ as in the last row of Table 7.2 .

Proposition 7.33. Let $\Sigma$ be an irreducible congruence of Seg-type two. For a general $L \in \Sigma$ with $\left(x_{1}, L, H_{1}\right),\left(x_{2}, L, H_{2}\right) \in F_{\Sigma}, x_{1} \in X_{1}, x_{2} \in X_{2}, H_{1} \in Y_{1}$ and $H_{2} \in Y_{2}$, we have that

$$
\begin{aligned}
\mathbb{T}_{X_{1}, x_{1}} \subset H_{2} & \text { and } & \mathbb{T}_{X_{2}, x_{2}} \subset H_{1}, \\
\mathbb{T}_{Y_{1}, H_{1}^{\vee}} \subset x_{2}^{\vee} & \text { and } & \mathbb{T}_{Y_{2}, H_{2}^{\vee}} \subset x_{1}^{\vee} .
\end{aligned}
$$

Proof. As in the proof of Proposition 7.26, we get surjections $\Phi_{i}: T_{\Sigma, L} \rightarrow T_{X_{i}, x_{i}}$ and $\Psi_{i}: T_{\Sigma, L} \rightarrow T_{Y_{i}, H_{i}^{\vee}}$ for $i \in\{1,2\}$, such that $\left.\varphi\right|_{\boldsymbol{x}_{\boldsymbol{i}}}=\left(\Phi_{i}(\varphi) \bmod \boldsymbol{L}\right)$ and $\left.\Psi_{i}(\varphi)^{*}\right|_{\boldsymbol{L}}=\left(\varphi \bmod \boldsymbol{H}_{\boldsymbol{i}}\right)$ for all $\varphi \in T_{L} \Sigma$. Let $\varphi_{i}: \boldsymbol{L} \rightarrow \mathbb{A}^{4} / \boldsymbol{L}$ be a homomorphism with $\operatorname{ker} \varphi_{i}=\boldsymbol{x}_{\boldsymbol{i}}$ and $\operatorname{im} \varphi_{i}=\boldsymbol{H}_{\boldsymbol{i}} / \boldsymbol{L}$. The image of $\Phi_{1}\left(\varphi_{1}\right): \boldsymbol{x}_{\mathbf{1}} \rightarrow \mathbb{A}^{4} / \boldsymbol{x}_{\mathbf{1}}$ is contained in $\boldsymbol{L} / \boldsymbol{x}_{\mathbf{1}}$ and the image of $\Phi_{1}\left(\varphi_{2}\right): \boldsymbol{x}_{\mathbf{1}} \rightarrow \mathbb{A}^{4} / \boldsymbol{x}_{\mathbf{1}}$ in $\boldsymbol{H}_{\mathbf{2}} / \boldsymbol{x}_{\mathbf{1}}$. This shows that $H_{2}$ is tangent to $X_{1}$ at $x_{1}$. Similarly, the images of $\Phi_{2}\left(\varphi_{1}\right)$ and $\Phi_{2}\left(\varphi_{2}\right)$ are contained in $\boldsymbol{H}_{\mathbf{1}} / \boldsymbol{x}_{\mathbf{2}}$ and $\boldsymbol{L} / \boldsymbol{x}_{\mathbf{2}}$, respectively, and $H_{1}$ is tangent to $X_{2}$ at $x_{2}$. Dually, the kernel of $\Psi_{1}\left(\varphi_{1}\right)^{*}: \boldsymbol{H}_{\mathbf{1}} \rightarrow \mathbb{A}^{4} / \boldsymbol{H}_{\mathbf{1}}$ contains $\boldsymbol{L}, \operatorname{ker} \Psi_{1}\left(\varphi_{2}\right)^{*} \supset \boldsymbol{x}_{\mathbf{2}}$, $\operatorname{ker} \Psi_{2}\left(\varphi_{1}\right)^{*} \supset \boldsymbol{x}_{\mathbf{1}}$, and $\operatorname{ker} \Psi_{2}\left(\varphi_{2}\right)^{*} \supset \boldsymbol{L}$.

In particular, we have shown $Y_{2} \subset X_{1}^{\vee}, Y_{1} \subset X_{2}^{\vee}, X_{2} \subset Y_{1}^{\vee}$, and $X_{1} \subset Y_{2}^{\vee}$. Moreover, in the situation of Proposition 7.33, if one of the components $X_{i}$ is a surface, then $L$ is a tangent line to $X_{i}$ at $x_{i}$. Dually, if a component $Y_{j}$ is a surface, then $L^{\vee}$ is a tangent line to $Y_{j}$ at $H_{j}^{\vee}$. This yields the following:

Corollary 7.34. Let $\Sigma$ be an irreducible congruence of Seg-type two.

- If $X_{1}$ and $X_{2}$ are two distinct curves, then $\Sigma$ consists of all lines intersecting both curves.

- If $X_{1}$ and $X_{2}$ are a curve and a surface, then $\Sigma$ consists of lines intersecting the curve that are also tangent to the surface.

- If $X_{1}$ and $X_{2}$ are two distinct surfaces, then $\Sigma$ consists of lines that are tangent to both surfaces.

- If $X_{1}=X_{2}$ is a curve, then $\Sigma$ is its secant congruence.

- If $X_{1}=X_{2}$ is a surface, then $\Sigma$ is its bitangent congruence. 
Finally, we want to show the converse of Corollary 7.34. Since we have already classified all strongly isotropic congruences and all congruences of Segtype one, we can still restrict ourselves to congruences of Seg-type two.

Proposition 7.35. Let $\Sigma \subset \operatorname{Gr}\left(1, \mathbb{P}^{3}\right)$ be an irreducible congruence of Seg-type two. In each of the following five cases, the components of $Y$ are projectively dual to the components of the strict focal locus X:

- If $\Sigma \subset \mathrm{CH}_{0}\left(C_{1}\right) \cap \mathrm{CH}_{0}\left(C_{2}\right)$ for two distinct curves $C_{1}$ and $C_{2}$, then these curves are the components $X_{1}, X_{2}$ of $X$.

- If $\Sigma \subset \mathrm{CH}_{0}(C) \cap \mathrm{CH}_{1}(S)$ for a curve $C$ and a surface $S$, then $C$ and $S$ are the components $X_{1}, X_{2}$ of $X$.

- If $\Sigma \subset \mathrm{CH}_{1}\left(S_{1}\right) \cap \mathrm{CH}_{1}\left(S_{2}\right)$ for two distinct surfaces $S_{1}$ and $S_{2}$, then these surfaces are the components $X_{1}, X_{2}$ of $X$.

- If $\Sigma \subset \operatorname{Sec}(C)$ for a curve $C$, then $X_{1}=X_{2}=C$.

- If $\Sigma \subset \operatorname{Bit}(S)$ for a surface $S$, then $X_{1}=X_{2}=S$.

Proof. In any of the five cases, Proposition 3.10 and particularly (3.3) imply the following: for $L \in \Sigma$ general, the tangent space $T_{\Sigma, L}$ is spanned by two homomorphisms $\varphi_{1}, \varphi_{2}: \mathbf{L} \rightarrow \mathbb{A}^{4} / \boldsymbol{L}$ with $\operatorname{ker} \varphi_{1}=\boldsymbol{x}_{\mathbf{1}}, \operatorname{im} \varphi_{1}=\boldsymbol{H}_{\mathbf{2}} / \boldsymbol{L}, \operatorname{ker} \varphi_{2}=\boldsymbol{x}_{\mathbf{2}}$, and $\operatorname{im} \varphi_{2}=\boldsymbol{H}_{\mathbf{1}} / \boldsymbol{L}$, where (for $i \in\{1,2\}$ )

- $L$ is either tangent to a surface $S$ at $x_{i}$ and $H_{i}=\mathbb{T}_{S, x_{i}}$

- or $L$ intersects a curve $C$ at $x_{i}$ and $H_{i}$ is spanned by $L$ and $\mathbb{T}_{C, x_{i}}$.

From this and Corollary 7.34 we also get immediately that the involved curves and surfaces are the components $X_{1}, X_{2}$ of $X$, as claimed in Proposition 7.35.

It is left to show that the components of $Y$ are indeed $X_{1}^{\vee}$ and $X_{2}^{\vee}$. For a congruence $\Sigma$ as in Proposition 7.35 , we know that $\Sigma^{\perp} \subset \operatorname{Gr}\left(1,\left(\mathbb{P}^{3}\right)^{*}\right)$ is also of Seg-type two and that $X\left(\Sigma^{\perp}\right)=Y(\Sigma)$. To see that the components of $Y(\Sigma)$ are $X_{1}^{\vee}$ and $X_{2}^{\vee}$, we use Theorem 3.13 in the first three cases of Proposition 7.35 and Theorem 5.2 together with Remark 5.23 in the last two cases.

A future line of research in this area could be to generalize the classification given in Table 7.2 to congruences in $\operatorname{Gr}\left(1, \mathbb{P}^{n}\right)$. An irreducible subvariety of $\operatorname{Gr}\left(1, \mathbb{P}^{n}\right)$ is a congruence if it has dimension and codimension $n-1$. Note that these generalized congruences are both isotropic and coisotropic by Corollary 4.5 and Lemma 7.3 . 



\section{Part II}

A Package for Tropical Geometry 



\section{Computing Tropical Varieties in Macaulay2}

This chapter introduces a package for doing tropical computations in Macaulay2 [45]. At the moment the main computational tool for tropical geometry is the program Gfan [54] by Jensen. This computes the Gröbner fan of an ideal $I$ and includes functions to compute only the subfan of the Gröbner fan given by the tropicalization of the variety $Z(I)$. The polyhedral geometry program Polymake [42] also has some tropical functionality that is not implemented in Gfan.

The package gfanInterface2 [52], implemented in Macaulay2, allows the user to interface with Gfan while retaining the computational speed provided by Macaulay2 for Gröbner basis computations. A drawback of this package is that it requires good knowledge of the functions and conventions of Gfan. The goal of the Tropical package is to provide a user friendly tool to do these computations in Macaulay2 without requiring any knowledge of these conventions. The package includes different strategies for the same function depending on the input, and calls functions from Gfan, via gfanInterface2, and Polymake, as appropriate. Moreover, the package implements some extra functionality not yet available in Gfan, such as computing multiplicities for tropical varieties of non-prime ideals and allowing the user to swap between the min and max conventions.

The package is available with the general release of Macaulay2 (since version 1.11). It can also be downloaded from

http://homepages. warwick.ac.uk/staff/D.Maclagan/papers/TropicalPackage.html

\subsection{Mathematical Background}

We follow the conventions of chapters two and three of [74]. Let $K$ be a field with valuation $\mathfrak{v}$.

Definition 8.1. Let $f=\sum_{u \in \mathbb{Z}^{n}} a_{u} x^{u}$ be a polynomial in $S=K\left[x_{1}^{ \pm 1}, \ldots, x_{n}^{ \pm 1}\right]$. The tropicalization of $f$ is the function $\operatorname{trop}(f): \mathbb{R}^{n} \rightarrow \mathbb{R}$ given by

$$
\operatorname{trop}(f)(w)=\min \left\{\mathfrak{v}\left(a_{u}\right)+w \cdot u \mid a_{u} \neq 0\right\} .
$$

The tropical hypersurface defined by $f$ is

$\operatorname{trop}(Z(f))=\left\{w \in \mathbb{R}^{n} \mid\right.$ the minimum in $\operatorname{trop}(f)(w)$ is achieved at least twice $\}$. 
Let $I$ be an ideal in $S$. The tropicalization of the variety $Z(I)$ is

$$
\operatorname{trop}(Z(I))=\bigcap_{f \in I} \operatorname{trop}(Z(f))
$$

The same definitions can be formulated using max instead of min. The Tropical package allows the user to choose their convention when loading the package.

If the ideal $I$ is generated by $f_{1}, \ldots, f_{s}$, it is not true in general that trop $Z(I)$ is the intersection of the tropical hypersurfaces associated to the $f_{i} \mathrm{~s}$. The intersection trop $Z\left(f_{1}\right) \cap \ldots \cap$ trop $Z\left(f_{s}\right)$ is a tropical prevariety.

Definition 8.2. Let $I=\left\langle f_{1}, \ldots, f_{s}\right\rangle$ be an ideal in $S=K\left[x_{1}^{ \pm 1}, \ldots, x_{n}^{ \pm 1}\right]$. Then $f_{1}, \ldots, f_{s}$ are a tropical basis of $I$ if trop $Z(I)=\bigcap_{i=1}^{s} \operatorname{trop} Z\left(f_{i}\right)$.

The tropical variety trop $Z(I)$ is a polyhedral complex ([74, Prop. 3.2.8]) contained in the Gröbner complex of the ideal $I$. If the valuation is trivial, the tropical variety is a rational polyhedral fan and is a subfan of the Gröbner fan of the ideal. Moreover, we can associate to each maximal cell an integer number, called multiplicity, such that a certain balancing condition holds (see [74, Def. 3.3.1]).

The Tropical package takes as input an ideal $I$ in a usual (non-Laurent) polynomial ring $K\left[x_{1}, \ldots, x_{n}\right]$. The tropical variety computed is the variety $Z(J) \subset\left(K^{*}\right)^{n}$ of the ideal $J=I K\left[x_{1}^{ \pm 1}, \ldots, x_{n}^{ \pm 1}\right]$.

\subsection{Examples}

In this section, we give explicit examples in order to give a short overview of the package. The computations are all over the field $Q$ of rational numbers with trivial valuation, hence all tropical varieties are polyhedral fans.

Example 8.3. Consider the algebraic variety $X=Z(I) \subset\left(\mathbb{C}^{*}\right)^{2}$ defined by the ideal $I=\langle x+y+1\rangle$. The tropicalization of this variety can be computed using the function tropicalVariety(I). The package outputs this as a tropical cycle: a fan with a list of multiplicities corresponding to integer weights on the maximal cones. We extract information about the tropical cycle using associated functions. For example, rays gives the generators of the rays as the columns of a matrix. 


\begin{tabular}{|c|c|}
\hline 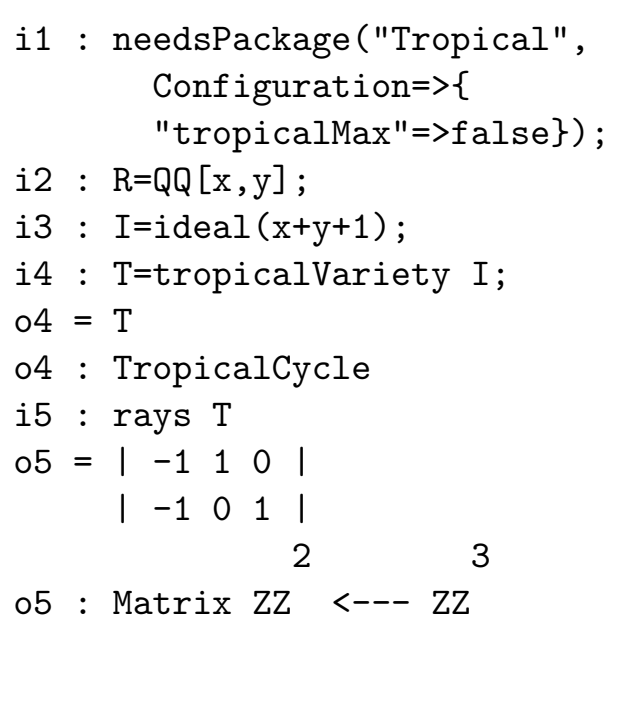 & 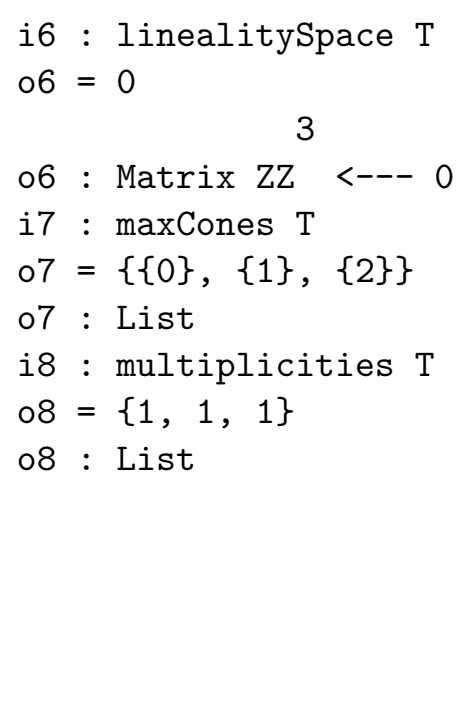 \\
\hline
\end{tabular}

The tropical variety trop $Z(I)$ is the standard tropical line in the plane: a onedimensional fan in $\mathbb{R}^{2}$ whose rays are $(-1,-1),(1,0)$, and $(0,1)$.

The function tropicalVariety uses one of two different algorithms depending on the input ideal. If the ideal is prime, the tropical variety is connected through codimension one ([9, Thm. 3.1]) and the Gfan commands gfan_tropicalstartingcone and gfan_tropicaltraverse, which implement the algorithm described in [9], are used. However if the ideal is not prime, this algorithm might fail. The package then calls the more computationally expensive command gfan_tropicalbruteforce, which computes the entire Gröbner fan. The multiplicities are then computed separately. The package does not require that the user knows these intricacies, but simply requires that they flag when the ideal is not prime.

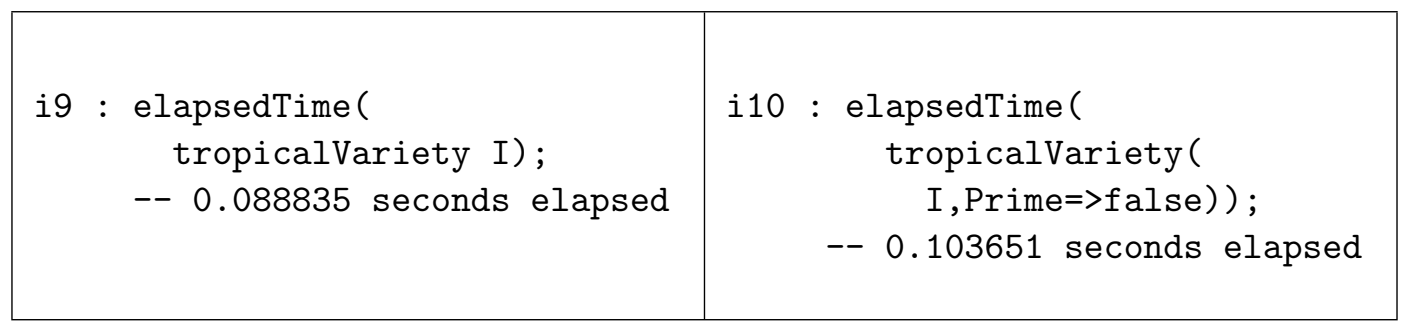

For most functions, Gfan requires the input to be homogeneous. The Tropical package will accept non-homogeneous input, and do the pre- and post-processing to put it into a format acceptable for $\mathrm{Gfan}$. Small additions such as this help decrease the prerequisite knowledge for the package.

Example 8.4. A tropical cycle is a fan with multiplicities attached to its maximal cones; it need not be the tropicalization of an algebraic variety. Therefore the package allows the user to create a tropical cycle manually by defining a fan via its maximal cones and attaching multiplicities to each of those cones. The following example shows how we can construct trop $Z(I)$ manually. 


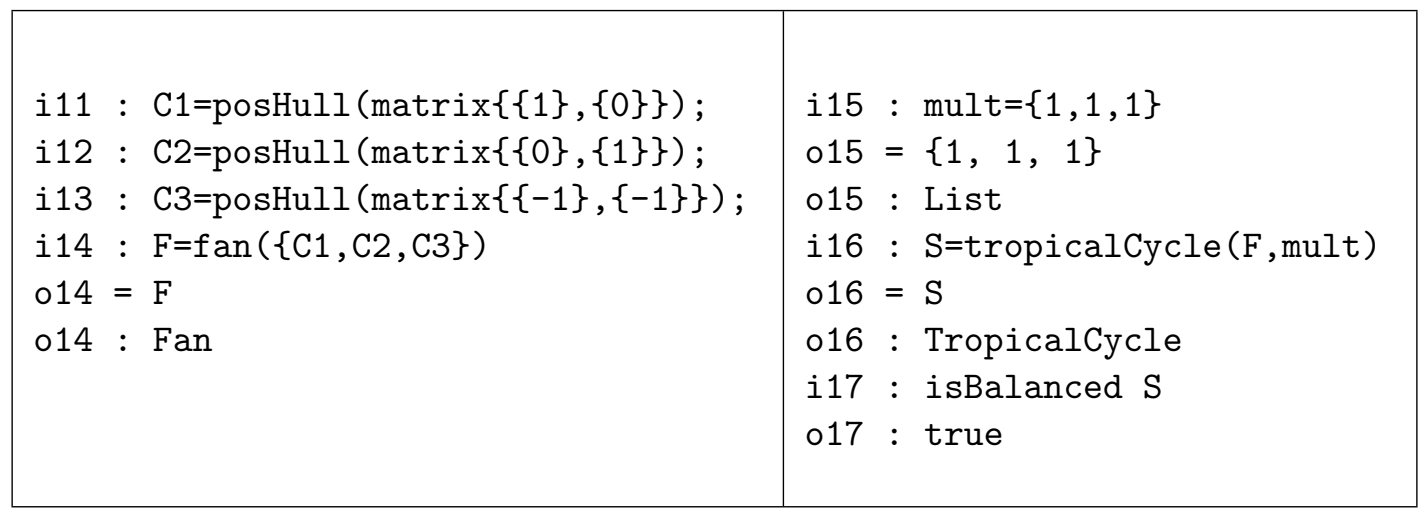

The tropicalCycle command does not check whether the resulting weighted fan is balanced. To verify this, we use the isBalanced command.

Example 8.5. Consider the tropical hypersurfaces trop $Z(f)$ and trop $Z(g)$ cut out by the polynomials $f=x+y+z$ and $g=x^{2}+y^{2}+z^{2}$. Their intersection cuts out a tropical prevariety. We would like to compute whether this prevariety is equal to the tropical variety trop $Z(I)$ where $I=\langle f, g\rangle$.

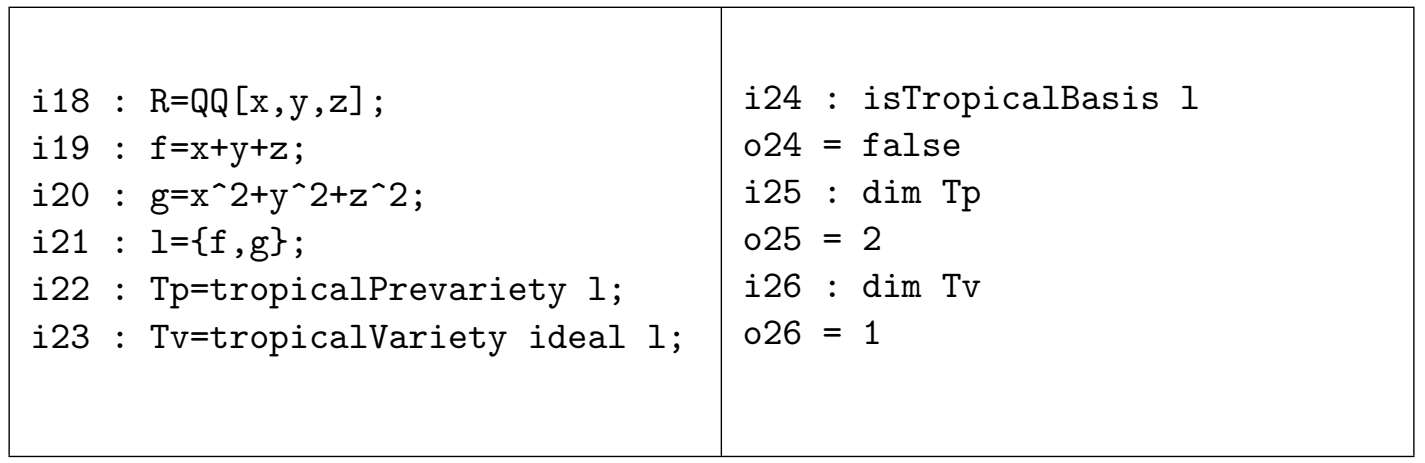

The polynomials $f, g$ are not a tropical basis for $I$ and therefore the prevariety given by them is not equal to trop $Z(I)$. We can see from our computation that the prevariety has a two-dimensional cone, while trop $Z(I)$ is one-dimensional.

Example 8.6. For two curves $Z(f)$ and $Z(g)$ in $\mathbb{P}^{2}$, Bézout's Theorem states that $|Z(f) \cap Z(g)|$ equals $\operatorname{deg}(f) \cdot \operatorname{deg}(g)$, counting multiplicities. The tropical analogue of Bézout's Theorem states that the stable intersection of trop $Z(f)$ and trop $Z(g)$ is $\operatorname{deg}(f) \cdot \operatorname{deg}(g)$ points, counting multiplicities. The following example shows how the package and the stableIntersection function can be used to verify examples of tropical Bézout's Theorem. 


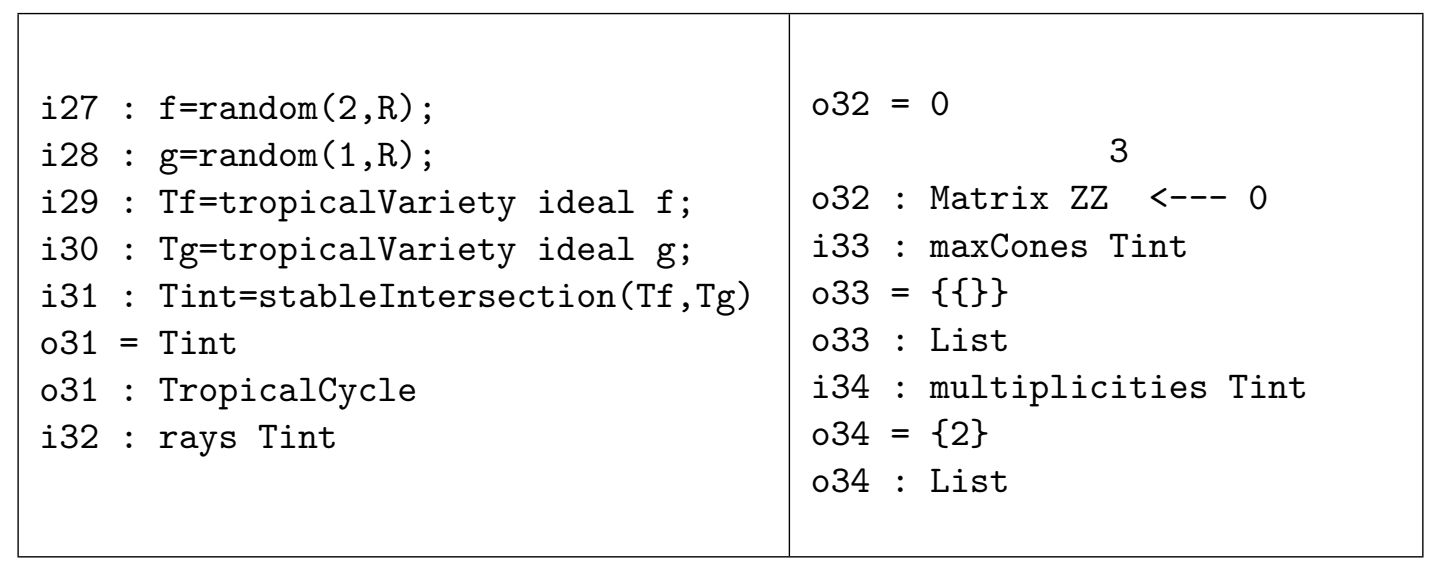

The above code considers the stable intersection of a tropical line and a plane quadric. The resulting tropical cycle is a single point, the origin, with multiplicity two, verifying the claim of tropical Bézout's theorem.

The function stableIntersection has two different strategies for computation depending on the software available to the user. If the user has a recent version of Polymake installed, the default strategy is to use atint [48], a Polymake extension for tropical intersection theory by Simon Hampe. If this is not available, the package instead uses Gfan to compute the stable intersection.

\subsection{Future Plans}

We plan for the Tropical package to become the umbrella package for all tropical computations in Macaulay2. This will include implementing alternate strategies for some of the core commands as algorithms improve, before they are included into Gfan and Polymake.

In addition, there are still functions available in Gfan and Polymake that are not yet available in the package. We particularly highlight the treatment of nontrivial valuations, which is available in Gfan, and the visualization of lowdimensional tropical varieties, which is available in Polymake. 



\section{Part III}

\section{A Simplicial Complex for Music Theory and Praxis}





\section{The Complex of Non-Chromatic Scales}

We consider the space of all musical scales with the ambition to systematize it. To do this, we pursue the idea to view certain scales as basic constituents and to "mix" all remaining scales from these. This chapter is written for readers who neither have a background in music nor mathematical knowledge exceeding basic linear algebra. The German version of this article appeared in Mitteilungen $\operatorname{der} D M V$, volume 25, issue 1.

The musical idea of using basic constituents for the space of all scales has been suggested in the recently published book [31] on improvisation (not only) in jazz music. From the mathematical point of view, these constituents form a simplicial complex, whose facets coincide with the most widely used scales in western music - with the exception of the blues scale. We will explain this connection in the following. First, we have to clarify what exactly we mean by a scale. The pitch space that is currently used in western music contains twelve different pitch classes: these are the seven natural notes $C, D, E, F, G, A, B$ as well as the five notes $C \sharp / D b, D \sharp / E b, F \sharp / G b, G \sharp / A b, A \sharp / B b$, which appear as raising/lowering of the naturals.

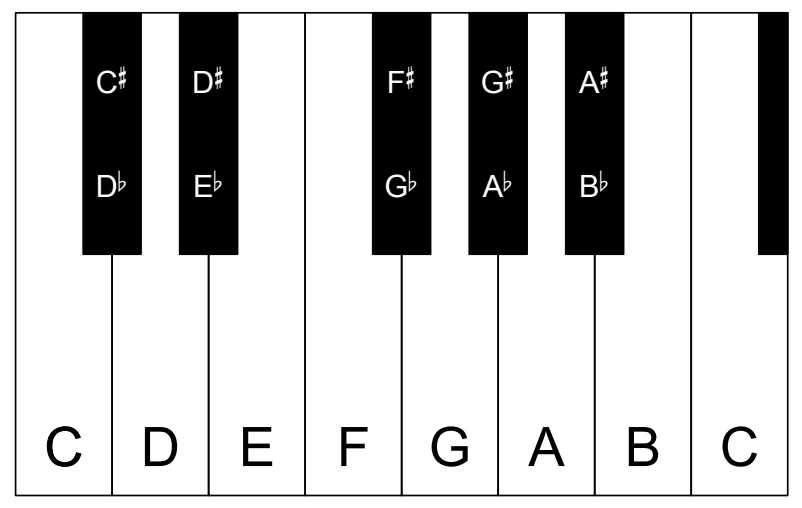

Figure 9.1: Western pitch space in modern times with twelve pitch classes.

From the mathematical perspective, we do not want to distinguish the notes $C \sharp$ and $D b$. In what follows, we will always use the $\sharp$-convention. A scale is simply defined to be a subset of $\{C, C \sharp, D, D \sharp, E, F, F \sharp, G, G \sharp, A, A \sharp, B\}$. As an example, we consider the $C$ major scale in our subset notation:

$$
\{C, D, E, F, G, A, B\} \text {. }
$$


The twelve notes have a cyclic order. For example, in the $C$ major scale we have again $C$ after the $B$ (see Fig. 9.2). Note that the two $C^{\prime}$ 's in Figure 9.1 are different pitches, but they are said to be in the same pitch class. This cyclic order allows us to define a distance between two pitch classes $t_{1}, t_{2} \in\{C, C \sharp, D, D \sharp, E, F, F \sharp, G, G \sharp, A, A \sharp, B\}$ : on the one hand, we can consider the clockwise distance from $t_{1}$ to $t_{2}$, and on the other hand the clockwise distance from $t_{2}$ to $t_{1}$. The distance between $t_{1}$ and $t_{2}$ is defined to be the minimum of both clockwise distances. As an example, we look at the pitch classes $A$ and $C$ : the clockwise distance from $A$ to $C$ is three and the clockwise distance from $C$ to $A$ is nine, which means that these two pitch classes have distance three. The sequence of distances between consecutive pitch classes in a scale in the cyclic order is called the interval sequence of the scale. As we can see in Figure 9.2, the $C$ major scale has the interval sequence 2-2-1-2-2-2-1.

The cyclic order of the twelve pitch classes implies that the interval sequence of a scale has a cyclic order, too. For example, we can also say that the $C$ major scale has the interval sequence 2-1-2-2-2-1-2. From the musical perspective, we would say that we start the major scale from a different root than $C$ - in this case $D$. This yields the widely used Gregorian modes, D-Dorian in our case. Mathematically speaking, we would identify the scales C-Ionian, D-Dorian, EPhrygian, F-Lydian, G-Mixolydian, A-Aeolian (natural A minor) and B-Locrian with each other, since they all have the same interval sequence. These modes are important in modal jazz. Since there are twelve major scales and thus $12 \cdot 7=84$ different Gregorian modes, the mentioned book by Deuker [31] takes the didactical approach to restrict oneself to the consideration of the major scales instead of studying all Gregorian modes, although this is still common in the widespread scale theory. A modern representative of the latter approach is for example Mark Levine [73].

An interval of length one is called semitone (or half tone) and an interval of length two is called whole tone. The scale whose intervals are twelve semitones (and thus contains all twelve pitch classes) is called chromatic scale. Therefore, we say that a scale whose interval sequence does not contain two consecutive semitones is non-chromatic. This is equivalent to the scale not containing three consecutive pitch classes in the cyclic order in Figure 9.2. The $C$ major scale is an example for such a non-chromatic scale. A counterexample is given by the scale

$$
\{C, C \sharp, E, F, G, A, B\},
$$

with interval sequence 1-3-1-2-2-2-1. With some justification, we can view the non-chromatic scales as the "primary colors" in music, whereas the (partly) chromatic scales with at least two consecutive semitones could be seen as "secondary colors". With this approach, the mentioned book [31] tries to order the space of all scales systematically.

Here we consider the non-chromatic scales from the mathematical perspective. The non-chromatic scales form a simplicial complex. Such a simplicial com- 


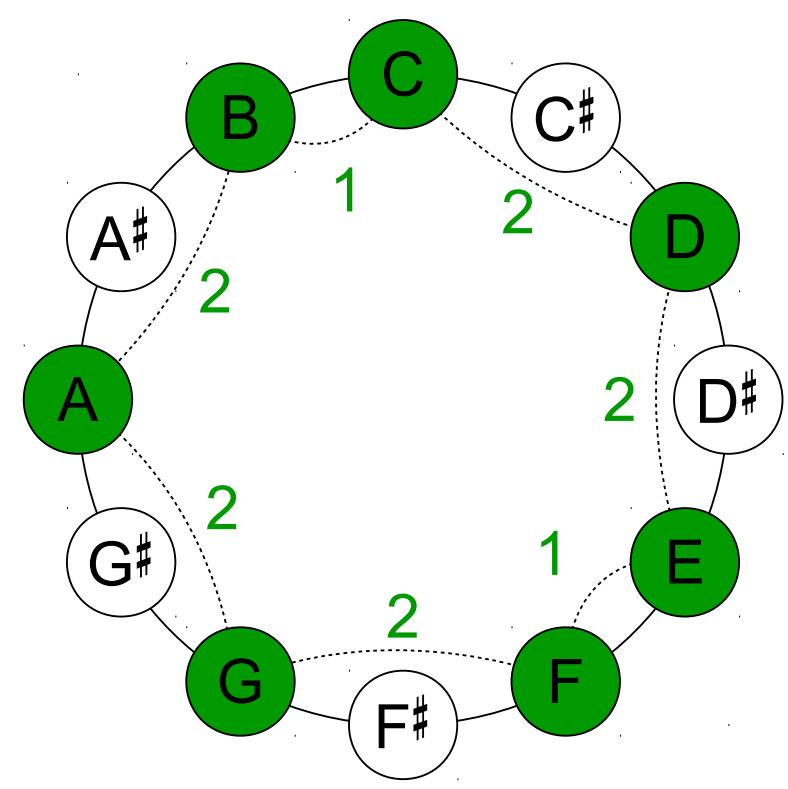

Figure 9.2: Cyclic order of the twelve pitch classes. Marked in green: $C$ major scale with interval sequence.

plex is defined on a ground set $\mathcal{G}$ as a set $\mathcal{K}$ of finite subsets of $\mathcal{G}$ such that, for every set $M$ in $\mathcal{K}$ and every subset $T$ of $M$, we have that $T$ is also in $\mathcal{K}$. As an example, we choose the ground set $\mathcal{G}:=\{0,1,2\}$. The set $\mathcal{K}_{1}:=\{\{0,1\},\{2\}\}$ is not a simplicial complex since $\{0\}$ is a subset of $\{0,1\}$ but not contained in $\mathcal{K}_{1}$. We can extend $\mathcal{K}_{1}$ to a simplicial complex in the following way: $\mathcal{K}_{2}:=\{\{0,1\},\{0\},\{1\},\{2\}, \varnothing\}$.

The set of all non-chromatic scales is a simplicial complex on the ground set of the twelve pitch classes $\mathcal{G}:=\{C, C \sharp, D, D \sharp, E, F, F \sharp, G, G \sharp, A, A \sharp, B\}$. Indeed, if we remove a pitch class from a non-chromatic scale, the scale stays nonchromatic. We denote this complex of non-chromatic scales in the following by $\mathcal{K}_{N C}$. In other words, we can define $\mathcal{K}_{N C}$ as the set of all subsets of the twelve pitch classes in Figure 9.2 which do not contain three consecutive pitch classes. In the following, we want to answer three questions concerning $\mathcal{K}_{N C}$ which mathematicians typically ask about a given simplicial complex. Moreover, we will see that these questions are musically relevant.

\section{$9.1 f$-Vector}

We can represent the sets with $n$ elements in a simplicial complex geometrically as $(n-1)$-dimensional objects. A set with one element corresponds to a point, a set with two elements to a line segment, a set with three elements to a triangle, a set with four elements to a tetrahedron, etc. This allows us to draw simplicial complexes (see Figure 9.3 for an illustration of the example complex $\mathcal{K}_{2}$ ). Such 
an illustration is purely schematic; for example, embeddability of the complex is not essential.

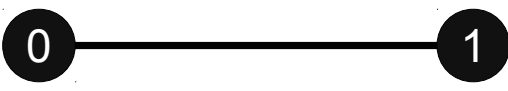

Figure 9.3: $\mathcal{K}_{2}$.

The higher-dimensional generalization of a tetrahedron is called simplex. This is where the name "simplicial complex" stems from. Now we consider a fixed simplicial complex and a positive integer $n$. We denote by $f_{n}$ the number of $n$-dimensional simplexes in $\mathcal{K}$ (i.e., the number of sets in $\mathcal{K}$ that have $n+1$ elements). We view the empty set $\varnothing$, which is contained in every simplicial complex, as a -1 -dimensional simplex and therefore we set $f_{-1}=1$. The list $\left(f_{-1}, f_{0}, f_{1}, f_{2}, f_{3}, \ldots\right)$ of all these numbers is the $f$-vector of $\mathcal{K}$. The $f$-vector of the example complex $\mathcal{K}_{2}$ is $(1,3,1)$.

When we ask for the $f$-vector of our simplicial complex $\mathcal{K}_{N C}$, we ask how many non-chromatic scales with $0,1,2,3, \ldots, 12$ pitch classes exist. This is relevant musically; for example, one might want to know how to improvise nonchromatically. In this case, it is particularly important to know how many pitch classes one is allowed to use maximally. Furthermore, there are several improvisational approaches which are built up on pentatonic scales - these are scales with five pitch classes. Therefore the number of non-chromatic pentatonic scales is relevant. We calculated the $f$-vector of $\mathcal{K}_{N C}$ using the software polymake [42] (it also possible to do it by hand without too much effort):

$$
\begin{aligned}
& (1, \quad 12,66,208,399,456,282,72,3) \\
& \left(f_{-1}, f_{0}, f_{1}, f_{2}, f_{3}, f_{4}, f_{5}, f_{6}, f_{7}\right) \text {. }
\end{aligned}
$$

In particular, this implies there there is no non-chromatic scale with nine or more pitch classes. Moreover, there are exactly three non-chromatic scales with eight pitch classes. We will have a closer look at these three scales in the next section. Furthermore, we note that there are 456 non-chromatic pentatonic scales.

\subsection{Facets}

A simplex in a simplicial complex $\mathcal{K}$ that is not contained in any other simplex of $\mathcal{K}$ is called facet. The example complex $\mathcal{K}_{2}$ has two facets, namely $\{0,1\}$ and $\{2\}$.

In musical terms, we want to know how many non-chromatic scales exist to which we cannot add a further pitch class without creating two consecutive semitones. One example for such a scale is the $C$ major scale. This concept 
of maximality is also important to improvising: it is enough to remember all maximal non-chromatic scales since all other non-chromatic scales are subsets of the maximal ones. This is the reason why the mentioned book by Deuker [31] treats these scales in detail. He even proves in the second chapter that there are exactly 57 maximal non-chromatic scales and outlines the significance of these scales in music history. It is remarkable that these scales are important in music as well as visible in the mathematical formulation (as facets of $\mathcal{K}_{N C}$ ).

In the following, we describe the 57 facets of $\mathcal{K}_{N C}$.

\begin{tabular}{clcr}
\hline $\begin{array}{c}\text { number of } \\
\text { pitch classes }\end{array}$ & interval sequence & $\begin{array}{c}\text { number of } \\
\text { scales }\end{array}$ & $\begin{array}{r}\text { name of } \\
\text { scales }\end{array}$ \\
\hline 8 & $2-1-2-1-2-1-2-1$ & 3 & diminished \\
7 & $2-2-1-2-2-2-1$ & 12 & major \\
7 & $2-1-2-2-2-2-1$ & 12 & melodic minor \\
7 & $2-1-2-2-1-3-1$ & 12 & harmonic minor \\
7 & $2-2-1-2-1-3-1$ & 12 & harmonic major \\
6 & $2-2-2-2-2-2$ & 2 & whole tone \\
6 & $1-3-1-3-1-3$ & 4 & augmented \\
\hline
\end{tabular}

Table 9.1: 57 maximal non-chromatic scales.

We see in Table 9.1 that the 57 maximal non-chromatic scales have only seven different interval sequences. Looking at the first row, there are three different scales which each have eight pitch classes and the prescribed interval sequence. These three scales are exactly those that we encountered when calculating the

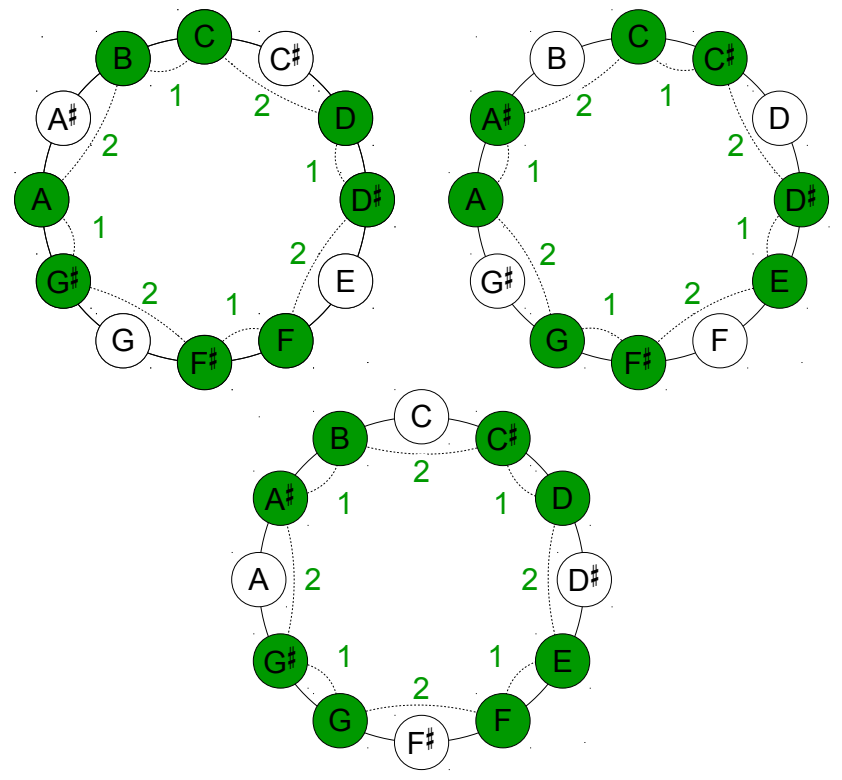

Figure 9.4: The three diminished scales.

$f$-vector. They are depicted in Figure 9.4. Furthermore, we see 48 maximal 
non-chromatic scales which consist of seven pitch classes. These come in four different types, determined by the interval sequence. The $f$-vector tells us that there are exactly $72=48+24$ non-chromatic scales with seven pitch classes. The remaining $24=3 \cdot 8$ scales are subsets of the three scales with eight pitch classes in Figure 9.4, each of those containing eight subsets with seven elements. Moreover, there are six maximal non-chromatic scales with six pitch classes. In particular, we see that the facets of our simplicial complex have different dimensions. Mathematically we say that $\mathcal{K}_{N C}$ is not pure.

Now we want to describe the meaning of these 57 facets in music.

2-1-2-1-2-1-2-1: This interval sequence has three different associated scales, see Figure 9.4. These are the diminished scales, also known as the octatonic scales, because they are the most widely used scales among all the scales with eight pitch classes. Nowadays they are frequently used in jazz. There is a whole jazztextbook devoted to these scales [106]. Around the year 1900, the diminished scales were quite popular in Russia, in particular in compositions by RimskiKorsakov. The Dutch composer Willem Pijper used these scales extensively as well. Thus, the diminished scales are sometimes also called Korsakovian scales or Pijper scales.

2-2-1-2-2-2-1: This interval sequence corresponds to the well-known major scale (today often called melodic major scale). Our first example of a scale was the $C$ major scale, but all twelve possibilities to choose a starting note yield twelve different major scales ( $D b$ major, $D$ major, etc.). These are the most widely used scales in western music. As already mentioned, the interval sequence above corresponds as well to the natural minor scales and the other Gregorian modes. For example, the $C$ major scale has exactly the same pitch classes as the natural $A$ minor scale. From the point of view of minor scales, we would write the interval sequence as 2-1-2-2-1-2-2. This variant of minor scales is the second most common scale in occidental music, directly after the major scales.

2-1-2-2-2-2-1: These are the melodic minor scales. There are again twelve different ones, depending on the chosen starting note. The melodic minor scales are widespread, from early compositions - particularly vocal music - to modern pop and rock. There is a common rule in books about the theory of harmony which says that the ascending melodic minor scale is the scale defined above, whereas the descending melodic minor scale is the same as the natural minor scale with the same starting note. This rule is uninteresting from the mathematical perspective, and Deuker [31] takes the view that it does not make much sense from the practical musical perspective either since specific melodies are rarely linearly ascending or descending.

2-1-2-2-1-3-1: This interval sequence corresponds to the harmonic minor scales. All twelve possible starting notes give twelve different such scales. In contrast to melodic minor scales, the harmonic minor scales are mainly used to build chords, but in particular in compositions by Mozart or Schubert they do appear in melodies as well.

2-2-1-2-1-3-1: This last type of seven-note maximal non-chromatic scales has again twelve different representatives. These are called harmonic major scales. 
They are conceived as a mixture of major and harmonic minor scales, and they are found most frequently in jazz music.

2-2-2-2-2-2: This is the whole tone scale. Due to its high symmetry, there are just two different such scales, depending on the starting note. Since there are no semitones at all, the choice of the starting note is a relatively arbitrary determination and there is no audible root in the scale. As a consequence, the impression of the whole tone scale is often described as levitating. In modern western music (except jazz), this scale is not used that often, but in impressionism - in particular in compositions by Debussy - it played a major role, and it was applied before this era by Franz Liszt and Rimski-Korsakow.

1-3-1-3-1-3: The augmented scales belong to this interval sequence. Analogously to the case of the diminished scales (see Fig. 9.4), there are four different augmented scales. These appeared already in compositions by Franz Liszt, but they were used increasingly in the 20th century by composers like Béla Bartók or Arnold Schönberg as well as in jazz. There is even a jazz-textbook which is explicitly dedicated to the augmented scales [89].

At the end of this section, we want to deduce why there are exactly the seven possibilities in Table 9.1 for an interval sequence $\mathcal{I}$ of a maximal non-chromatic scale.

Observation 1: $\mathcal{I}$ cannot contain an interval of length four or more. If $\mathcal{I}$ would have such an interval -e.g., between the pitch classes $C$ and $E$-, then we could add a pitch class in the middle of the interval ( $D$ in the example) and the scale would stay non-chromatic.

Observation 2: If $\mathcal{I}$ contains an interval of length three, then the right and the left neighbor of this interval in $\mathcal{I}$ must be both semitones. In other words, if 3 is contained in $\mathcal{I}$, then $\mathcal{I}$ has to contain the sequence 1-3-1. Indeed, if $\mathcal{I}$ would contain the sequence 3-2 - e.g., the pitch classes $C, D \sharp$ and $F$ are in the scale -, then we could add a pitch class from the interval of length three (in the example $D)$ to the scale such that the scale would stay non-chromatic.

Using these two observations, we can distinguish a few cases to explore all seven possibilities for $\mathcal{I}$.

Case 1: $\mathcal{I}$ contains a 3 and thus - by Observation 2 - the sequence 1-3-1. Either we extend this sequence with a 3 or with a 2 . If we choose to extend it with a 3 , we see that $\mathcal{I}$ has to contain the sequence 1-3-1-3-1. Since all numbers in $\mathcal{I}$ have to add up to twelve, there is just one possibility to extend the sequence to an interval sequence of a non-chromatic scale:

$$
1-3-1-3-1-3
$$

If we would have chosen to extend the sequence 1-3-1 on the left side with a 3 , the same interval sequence would have been our result. Hence, we have just one case left to consider, namely that $\mathcal{I}$ contains the sequence $2-1-3-1-2$. By Observation 2, we have to complete this sequence with a 2 and a 1, which yields two possibilities:

$$
2-2-1-2-1-3-1
$$




$$
2-1-2-2-1-3-1
$$

Case 2: $\mathcal{I}$ contains no 3 , and as a consequence, consists only of ones and twos. Since the sum of all numbers in $\mathcal{I}$ has to be twelve, the number of ones in $\mathcal{I}$ is even. If $\mathcal{I}$ contains no ones at all, we know that $\mathcal{I}$ looks as follows:

$$
2-2-2-2-2-2
$$

If $\mathcal{I}$ contains exactly two ones, then there are only two possibilities to arrange them:

$$
\begin{aligned}
& 2-1-2-2-2-2-1 \\
& 2-2-1-2-2-2-1
\end{aligned}
$$

Due to the cyclic order, the further possibilities 2-2-2-1-2-2-1 and 2-2-2-2-1-2-1 are in fact equal to the two interval sequences above. If $\mathcal{I}$ contains four ones, it is already uniquely determined:

$$
2-1-2-1-2-1-2-1
$$

Moreover, this shows that $\mathcal{I}$ cannot contain more than four ones.

\subsection{Topology}

Topology is a branch of mathematics that investigates which properties of spaces are preserved under continuous deformations. We say that a deformation is continuous if - roughly speaking - it does not require to cut or glue parts of the space. Typical examples of continuous deformations are stretching and bending of spaces. For example, a ball can be continuously deformed to a cube. Since this deformation can also be reversed continuously, we say that ball and cube are homeomorphic. A mug with a handle is homeomorphic to a donut with a hole. However, such a donut is not homeomorphic to a ball, because its hole cannot be filled by a continuous deformation. Thus, the number of holes is an example of a property that homeomorphic spaces have in common.

The idea of homeomorphy can be extended to homotopy equivalence, which also preserves the number of holes. Let $X$ and $Y$ be two spaces with two continuous maps $g: X \rightarrow Y$ and $f: Y \rightarrow X$. If the compositions $f \circ g: X \rightarrow X$ and $g \circ f: Y \rightarrow Y$ are the identities on $X$ and $Y$, respectively (i.e., $f(g(x))=x$ for all $x \in X$ and $g(f(y))=y$ for all $y \in Y)$, then $X$ and $Y$ are called homeomorphic. If the map $f \circ g$ can be deformed continuously to the identity on $X$ and if $g \circ f$ can be deformed continuously to the identity on $Y$, then we say that $X$ and $Y$ are homotopy equivalent. Hence, every homeomorphism is a homotopy equivalence. Examples of homotopy equivalences that are not homeomorphisms are to thicken or to squash spaces. For instance, a ball and a point are homotopy equivalent but not homeomorphic. 
Holes in Simplicial Complexes. We can also count holes in a given simplicial complex $\mathcal{K}$. For this, we determine the (reduced) simplicial homology of the complex. We give here a short introduction to this topic and refer the interested reader to [51]. As in the definition of the $f$-vector, we consider all $n$-dimensional simplexes in $\mathcal{K}$. A formal sum over these simplexes denotes simply a sum of the simplexes with rational coefficients, which we write down without evaluating it. We denote by $C_{n}(\mathcal{K})$ the set of all such formal sums. This is a vector space over the rational numbers $Q$. In our example complex $\mathcal{K}_{2}$, we have among others the following formal sums:

$$
\frac{3}{2}\{0,1\} \in C_{1}\left(\mathcal{K}_{2}\right), 2\{0\}-\{2\} \in C_{0}\left(\mathcal{K}_{2}\right),-\varnothing \in C_{-1}\left(\mathcal{K}_{2}\right) .
$$

The boundary operator maps an $n$-dimensional simplex to its $(n-1)$-dimensional boundary. It maps, for instance, a triangle to its three bounding line segments, and a tetrahedron to its four faces (which are triangles). Formally, the boundary operator $\partial_{n}: C_{n}(\mathcal{K}) \rightarrow C_{n-1}(\mathcal{K})$ is defined by mapping every set in $\mathcal{K}$ with $(n+1)$ elements to an alternating sum of its subsets with $n$ elements. For this, the elements of a set with $(n+1)$ elements will be omitted one after the other, in an order that was fixed beforehand. In our example $\mathcal{K}_{2}$ this means:

$$
\begin{array}{r}
\partial_{1}(\{0,1\})=\{1\}-\{0\}, \\
\partial_{0}(\{0\})=\partial_{0}(\{1\})=\partial_{0}(\{2\})=\varnothing .
\end{array}
$$

The formal sums in $C_{n}(\mathcal{K})$ that get mapped to 0 by the boundary operator are called $n$-cycles. The set of all these cycles is a vector space, which we denote by $Z_{n}(\mathcal{K})$. We call the image $\partial_{n+1}(\Sigma)$ of a formal sum $\Sigma \in C_{n+1}(\mathcal{K})$ an $n$-boundary. The set of all these boundaries is also a vector space, denoted $R_{n}(\mathcal{K})$. Now one can verify immediately that every $n$-boundary is an $n$-cycle, i.e., $R_{n}(\mathcal{K}) \subseteq Z_{n}(\mathcal{K})$. We say that two $n$-cycles are equivalent if their difference is an $n$-boundary. The equivalence class of an $n$-cycle $\zeta$ is the set of all $n$-cycles that are equivalent to $\zeta$. We denote by $H_{n}(\mathcal{K})$ the set of all equivalence classes. This is again a vector space, called the $n$-th homology group of $\mathcal{K}$.

In our example $\mathcal{K}_{2}$, the 0 -cycles are all formal sums of the form $a\{0\}+b\{1\}+$ $c\{2\}$, where $a, b, c$ are rational numbers with $a+b+c=0$. The 0 -boundaries look as follows: $d\{0\}-d\{1\}$ with $d \in \mathbb{Q}$. Thus, the equivalence class of a 0 cycle $a\{0\}+b\{1\}+c\{2\}$ consists of all 0 -cycles that have the same coefficient $c$ in front of $\{2\}$. Hence, such an equivalence class is uniquely determined by the coefficient $c$. Since $c$ is an arbitrary rational number, we say that $H_{0}\left(\mathcal{K}_{2}\right)$ is isomorphic to $\mathbb{Q}$. The only 1 -cycle of $\mathcal{K}_{2}$ is 0 (i.e., the formal sum with coefficients $0)$. Due to the fact that $\mathcal{K}_{2}$ contains no set with 3 elements, we can say that 0 is also the only 1-boundary of $\mathcal{K}_{2}$. This shows that $H_{1}\left(\mathcal{K}_{2}\right)$ contains nothing but 0 .

When considering the illustration of $\mathcal{K}_{2}$ in Figure 9.3, we see that $\mathcal{K}_{2}$ is not connected. This means that one cannot draw $\mathcal{K}_{2}$ without lifting the pen. Instead, $\mathcal{K}_{2}$ has two connected components. We can read off the number of these components from the zeroth homology group: for every simplicial complex $\mathcal{K}$, 
the vector space $H_{0}(\mathcal{K})$ is isomorphic to the vector space $\mathbb{Q}^{k-1}$, where $k$ is the number of connected components of $\mathcal{K}$. We use the convention that $\mathbb{Q}^{0}=\{0\}$.

We cannot only count the connected components of a simplicial complex with homology, but also the number of holes. To explain this, we consider a filled and an unfilled triangle as example complexes:

$$
\begin{aligned}
& \mathcal{K}_{\Delta}:=\{\{0,1\},\{1,2\},\{0,2\},\{0\},\{1\},\{2\}, \varnothing\}, \\
& \mathcal{K}_{\mathbf{\Delta}}:=\{\{0,1,2\},\{0,1\},\{1,2\},\{0,2\},\{0\},\{1\},\{2\}, \varnothing\} .
\end{aligned}
$$

The illustration of both complexes looks as follows:
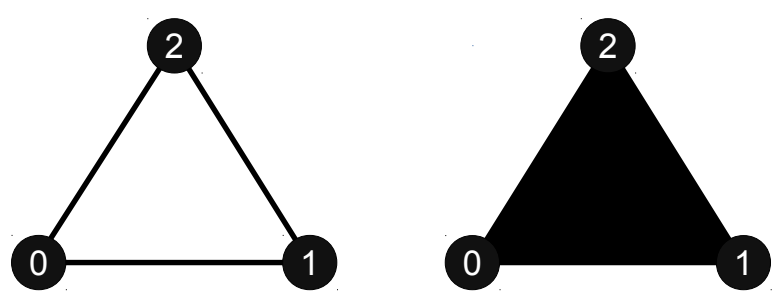

Figure 9.5: $\mathcal{K}_{\triangle}$ and $\mathcal{K}_{\Delta}$.

Since both triangles are connected, we know that $H_{0}\left(\mathcal{K}_{\Delta}\right)$ and $H_{0}\left(\mathcal{K}_{\mathbf{\Delta}}\right)$ must be isomorphic to $\{0\}$. We can verify this directly: both for $\mathcal{K}_{\Delta}$ and for $\mathcal{K}_{\mathbf{\Lambda}}$, the first two boundary operators are equal:

$$
\begin{array}{r}
\partial_{1}(\{0,1\})=\{1\}-\{0\}, \\
\partial_{1}(\{1,2\})=\{2\}-\{1\}, \\
\partial_{1}(\{0,2\})=\{2\}-\{0\}, \\
\partial_{0}(\{0\})=\partial_{0}(\{1\})=\partial_{0}(\{2\})=\varnothing .
\end{array}
$$

Thus, all 0-cycles of both simplicial complexes are of the form $a\{0\}+b\{1\}+$ $c\{2\}$, where $a, b, c$ are rational numbers with $a+b+c=0$. The 0 -boundaries look like

$$
\begin{array}{r}
d(\{1\}-\{0\})+e(\{2\}-\{1\})+f(\{2\}-\{0\}) \\
=(-d-f)\{0\}+(d-e)\{1\}+(e+f)\{2\}
\end{array}
$$

with $d, e, f \in \mathbb{Q}$. From this we deduce that every 0 -cycle is a 0 -boundary. Hence, there is just one equivalence class of 0 -cycles, and $H_{0}\left(\mathcal{K}_{\Delta}\right)=H_{0}\left(\mathcal{K}_{\mathbf{\Delta}}\right)$ is a set with one element, which is consequently isomorphic to $\{0\}$, as claimed above.

The 1 -cycles of $\mathcal{K}_{\Delta}$ and $\mathcal{K}_{\mathbf{\Delta}}$ are of the form $d\{0,1\}+d\{1,2\}-d\{0,2\}$. The boundary operator $\partial_{2}$ of the filled triangle $\mathcal{K}_{\mathbf{\Delta}}$ maps $\{0,1,2\}$ to $\{1,2\}-\{0,2\}+$ $\{0,1\}$. That is why the 1-boundaries of $\mathcal{K}_{\mathbf{\Lambda}}$ look exactly like the 1 -cycles, and $H_{1}\left(\mathcal{K}_{\mathbf{\Lambda}}\right)$ contains just one equivalence class. We conclude again that $H_{1}\left(\mathcal{K}_{\mathbf{\Lambda}}\right)$ is isomorphic to $\mathbb{Q}^{0}$. Since the unfilled triangle $\mathcal{K}_{\triangle}$ contains no sets with three 
elements, the only 1-boundary of $\mathcal{K}_{\Delta}$ is 0 . Therefore, different 1 -cycles of $\mathcal{K}_{\Delta}$ can never be equivalent. We get that $H_{1}\left(\mathcal{K}_{\Delta}\right)$ is isomorphic to $\mathbb{Q}^{1}$, because the coefficient $d$ of the 1 -cycles is an arbitrary rational number. The triangle $\mathcal{K}_{\triangle}$ has a (one-dimensional) hole, whereas $\mathcal{K}_{\mathbf{\Delta}}$ has no holes. Thus, we have for both triangles that the dimensions of $H_{1}\left(\mathcal{K}_{\Delta}\right)$ and $H_{1}\left(\mathcal{K}_{\mathbf{\Lambda}}\right)$ are equal to the number of one-dimensional holes of $\mathcal{K}_{\Delta}$ and $\mathcal{K}_{\mathbf{\Lambda}}$, respectively.

Furthermore, we see in Figure 9.3 that $\mathcal{K}_{2}$ has no one-dimensional holes, which fits to our calculation that $H_{1}\left(\mathcal{K}_{2}\right)=\{0\}$. We can generalize this idea: intuitively, the dimension of $H_{n}(\mathcal{K})$ counts the $n$-dimensional holes in a simplicial complex $\mathcal{K}$.

Holes in $\mathcal{K}_{N C}$. Now one might ask the question how many holes of which dimensions are in the complex of non-chromatic scales, and what is the musical meaning of these holes? Using the software polymake, we obtain that $H_{5}\left(\mathcal{K}_{N C}\right)$ is isomorphic to $\mathbb{Q}^{3}$ and that all other homology groups are simply $\{0\}$. As a consequence, we can conclude that $\mathcal{K}_{N C}$ has three 5-dimensional holes. Moreover, the software polymake gives us a basis for the homology for each of the three holes, i.e., a set of hexatonic (6-note) scales that defines the boundary of the hole. Furthermore, we can check with polymake that the union of those hexatonic scales that define the boundary of such a hole is homeomorphic to the 5 -sphere. The $n$-sphere is the boundary of an $(n+1)$-dimensional ball. For instance, the unfilled triangle in Figure 9.5 is homeomorphic to the 1-sphere, which is simply the boundary of a circle. Roughly speaking, we can imagine the complex of non-chromatic scales to be composed of three 5-spheres.

Such a 5-sphere consists - as mentioned above - of hexatonic scales. Additionally, all subscales of such a hexatonic scale lie also on the respective sphere; just as the unfilled triangle in Figure 9.5 consists of sets with two elements, but their subsets with one element lie as points on the triangle. Now we still have scales with seven and eight pitch classes in $\mathcal{K}_{N C}$. We explain why these do not play any role in the topological picture of the three spheres using so-called collapses (see e.g. [68]). Whenever a simplicial complex $\mathcal{K}$ has a facet $F$ with a subset $M \subseteq F$ such that the following two properties hold:

1. $M$ contains exactly one element less than $F$,

2. $F$ is the only facet that contains $M$,

then we can remove $F$ and $M$ from the complex $\mathcal{K}$ and we obtain another simplicial complex. This process is referred to as a collapse and it is an example for a homotopy equivalence of simplicial complexes. In particular, the homology groups of the smaller complex are isomorphic to the homology groups of $\mathcal{K}$, and both simplicial complexes have the same number of holes. Let us observe the example in Figure 9.6 to understand better how a collapse looks and why it leaves the number of holes unchanged.

In our complex of non-chromatic scales, we remove successively all scales with seven and eight pitch classes by using collapses. For example, if we re- 


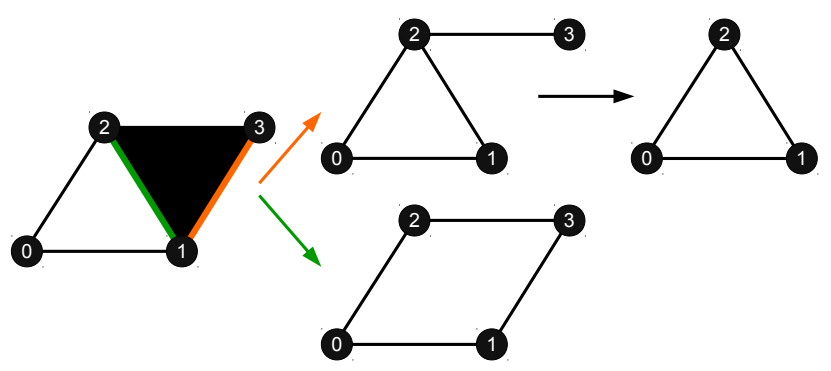

Figure 9.6: The left simplicial complex has 3 facets, but only one of these can be collapsed: $\{1,2,3\}$. In doing so, we can either remove the inner edge (green) or one of the two outer edges (e.g., orange). In the first case, we cannot perform a second collapse after the first one. In the second case, there is exactly one further collapse possible.

move the pitch class $C$ from the first eight-note scale in Figure 9.4, then we obtain a seven-note scale which can be extended to an eight-note non-chromatic scale in exactly one way. Thus, we can perform a collapse with these two scales. We consider the $C$ major scale as a second example. Here we can remove the pitch class $D$ such that the resulting hexatonic scale is contained in only one maximal non-chromatic scale. Therefore, we can collapse the $C$ major scale and its hexatonic subscale. In this manner, we can remove all scales with seven and eight pitch classes from $\mathcal{K}_{N C}$. This means that there are higherdimensional scales (namely with seven and eight pitch classes) glued onto the three 5-spheres in $\mathcal{K}_{N C}$ (which have hexatonic scales in their boundaries), just as the filled triangle is glued onto the unfilled triangle in Figure 9.6. By the way, it is impossible to collapse the hexatonic facets of $\mathcal{K}_{N C}$.

We are left with the question how our three spheres look exactly. In particular, we want to understand which hexatonic scales define the boundary of which sphere. For this, we consider the augmented triad $C-E-G \sharp$. After removing this triad from our twelve pitch classes, we obtain the nine-note scale in Figure 9.7 which was used by the French composer Messiaen.

This scale does not contain non-chromatic scales with seven or eight pitch classes, but 27 non-chromatic scales with six pitch classes. The latter scales can be obtained as follows: choose two pitch classes out of $C \sharp-D-D \sharp$ (there are three possibilities to do this), then pick two pitch classes from $F-F \sharp-G$ (again, there are three possibilities), and finally choose two pitch classes out of $A-A \sharp-B$ (which gives three more possibilities). Our analysis with polymake shows that these 27 hexatonic scales (and their subscales) form one of our three 5-spheres.

As in the case of the three different diminished scales in Figure 9.4, we see that there are four different Messiaen scales with nine pitch classes, which are obtained by omitting an augmented triad (see Figure 9.10). Each of these four scales contains 27 non-chromatic hexatonic scales, which form a sphere in $\mathcal{K}_{N C}$. However, we claimed before that there are only three holes in $\mathcal{K}_{N C}$. The rea- 


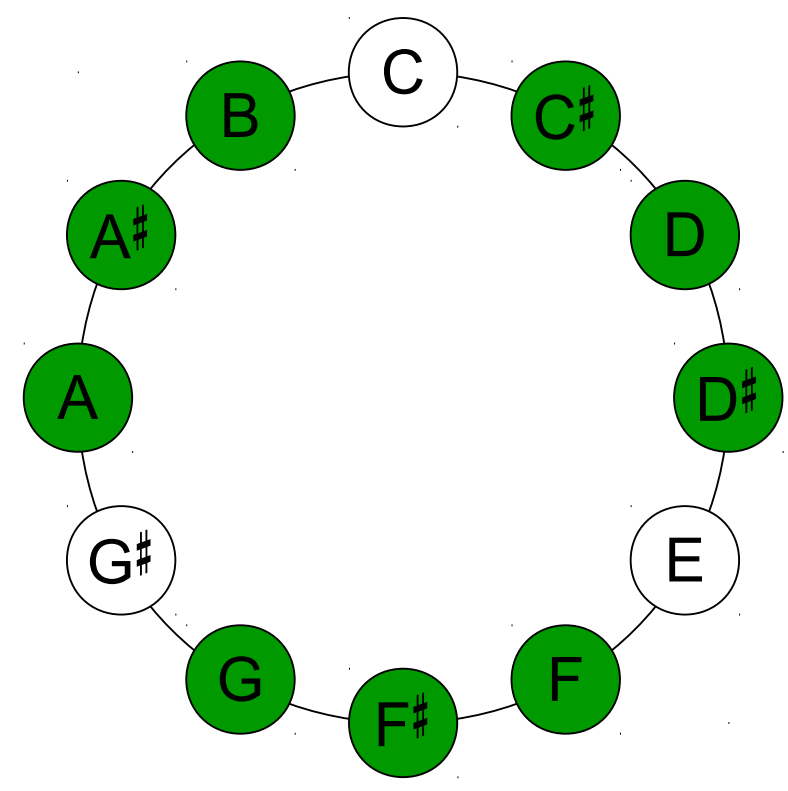

Figure 9.7: Messiaen's nine-note scale.

son for this is that the four Messiaen spheres described above are not independent of each other in $\mathcal{K}_{N C}$. This principle can be easily explained by looking at the skeleton of a tetrahedron: whenever we draw the simplicial complex in Figure 9.8, we see only three one-dimensional holes, although there are four 1-spheres (unfilled triangles).

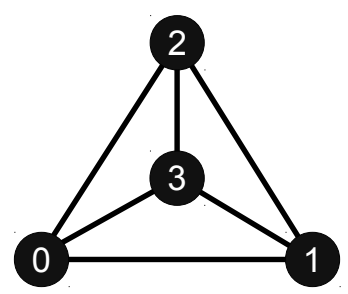

Figure 9.8: Simplicial complex with facets $\{0,1\},\{0,2\},\{0,3\},\{1,2\},\{1,3\}$, $\{2,3\}$.

One can show that any three of the four Messiaen spheres are indeed independent of each other in $\mathcal{K}_{N C}$, i.e., that they form a basis of the vector space $H_{5}\left(\mathcal{K}_{N C}\right)$. Let us finally observe that each 5-sphere described above contains exactly three of the hexatonic facets of $\mathcal{K}_{N C}$. In fact, any two distinct Messiaen scales intersect in a hexatonic facet of $\mathcal{K}_{N C}$, and all six hexatonic facets come as such an intersection (see Figure 9.10). Three distinct Messiaen scales intersect in an augmented triad, i.e., in a filled triangle as in Figure 9.5. In particular, we can successively perform collapses on the intersection of any two Messiaen spheres 
to obtain the common augmented triad, and afterwards we can collapse this triad until there is just a point left. That is why we can say from a topological perspective that the three spheres are glued together at a point. Hence, we can think of $\mathcal{K}_{N C}$ as three 6-dimensional balloons, which are held together at one point and have a higher-dimensional layer glued onto them (see Figure 9.9).

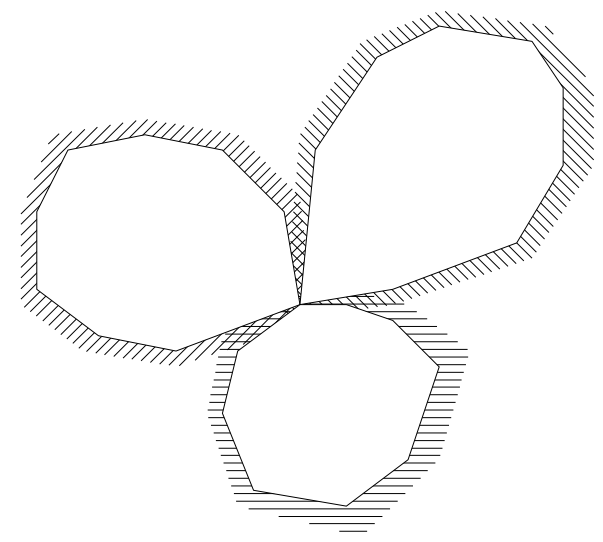

Figure 9.9: Schematic illustration of the topology of $\mathcal{K}_{N C}$.

We have described the 57 facets of the simplicial complex $\mathcal{K}_{N C}$ as well as its topological structure from the point of view of mathematics and music. In Mitteilungen der DMV, volume 22, issue 4, there is an article on "Mathematik und Musik?" written by the mathematician and pianist Christian Krattenthaler, which holds the opinion that mathematics and music have actually nothing to do with each other. However, we came to the conclusion that this is not always the case, since the simplicial complex $\mathcal{K}_{N C}$ has both mathematical and musical meaning, and there are many more connections between musical practice (e.g., change of scales) and mathematics that can be investigated. 


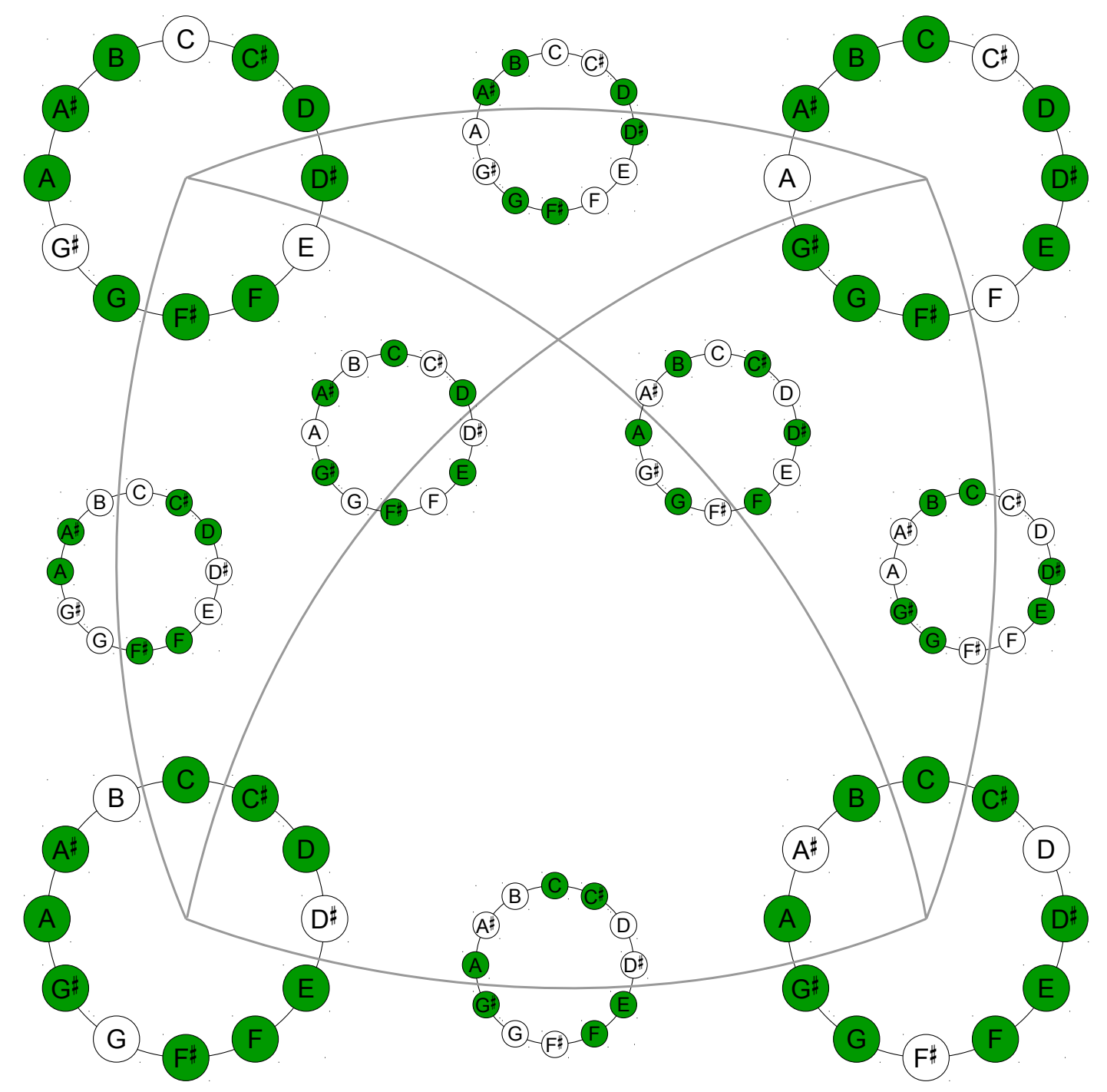

Figure 9.10: The hexatonic facets of $\mathcal{K}_{N C}$ are the pairwise intersections of the four Messiaen scales. 



\section{Appendix}

Here we present Macaulay2 code for computations in our examples and remarks in Part I, This code can also be found at

https://github.com/kathlenkohn/thesis-material

in the folder appendix. At several places, we use the package Coisotropy, which is also provided at this URL. We specify the computation time for each line of code which needed more than one second when executed on the following machine:

- CPU: Intel Core i7-5600U, $2.60 \mathrm{GHz}$

- RAM: DDR3, 16 GB

Code A. We use the package Coisotropy (see also Section 3.9) to verify the computations in Example 3.6.

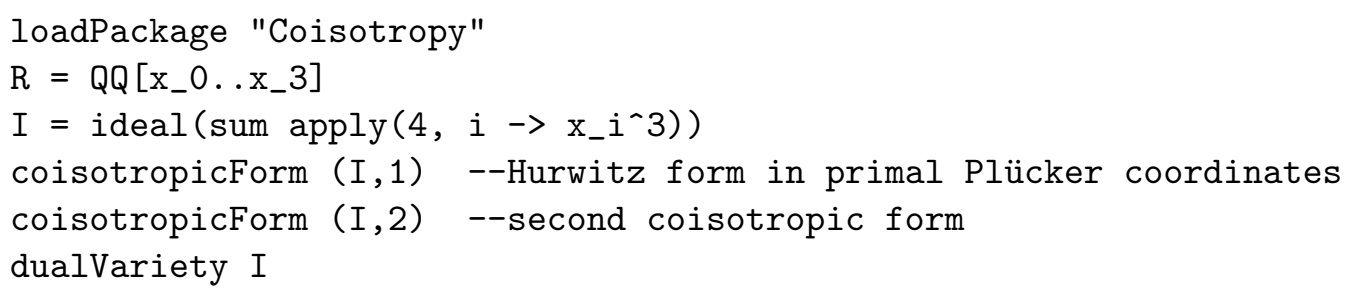

The following code verifies that the polynomial $(2.5)$ is indeed the Hurwitz form as computed above:

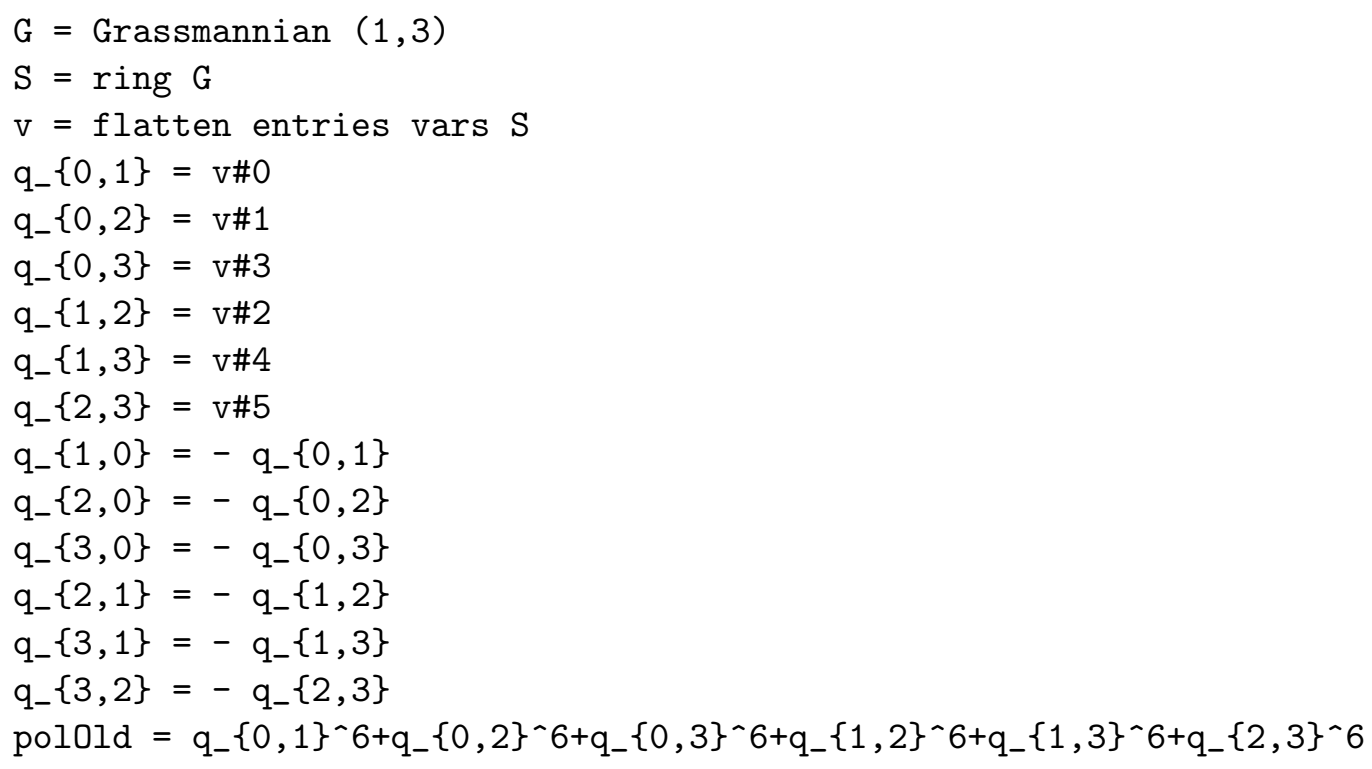




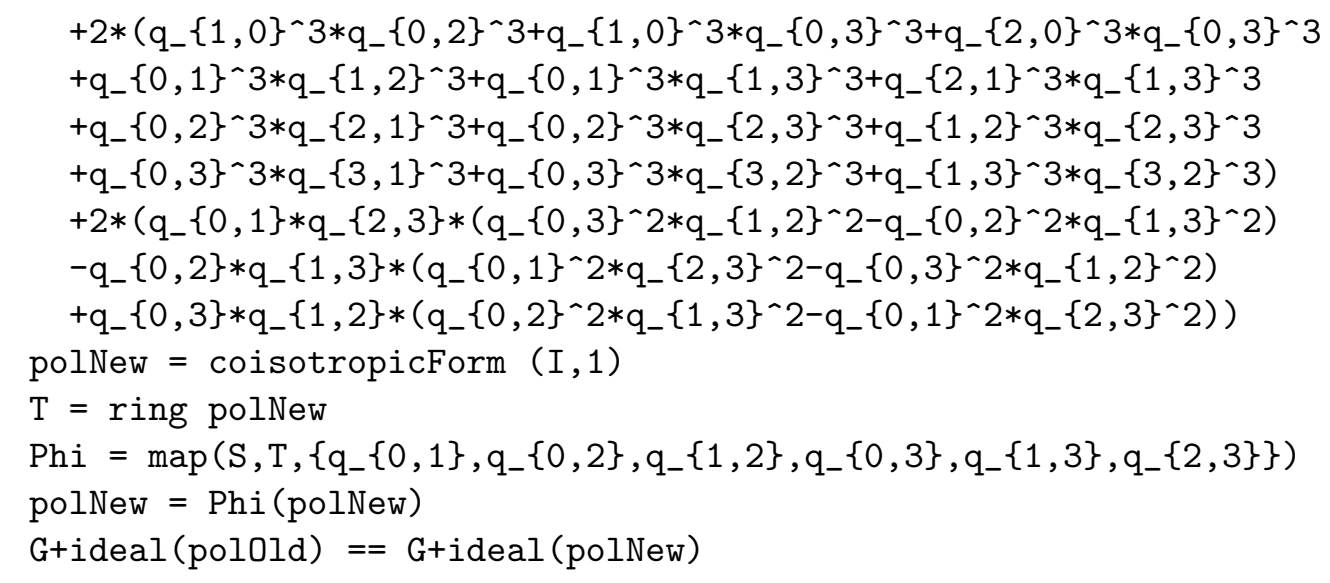

Code B. We use the package Coisotropy (see Section 3.9) to verify the Hurwitz form of the Segre variety $\mathbb{P}^{1} \times \mathbb{P}^{1}$ as stated in Example 3.16 .

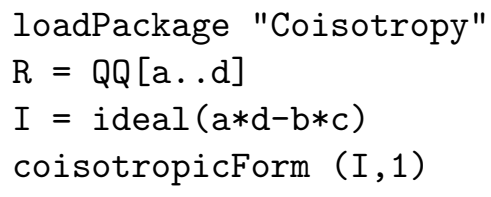

Code C. We use the package Coisotropy (see Section 3.9) to verify the Chow form of the Segre variety $\mathbb{P}^{1} \times \mathbb{P}^{2}$ as stated at the very end of Example 3.23 .

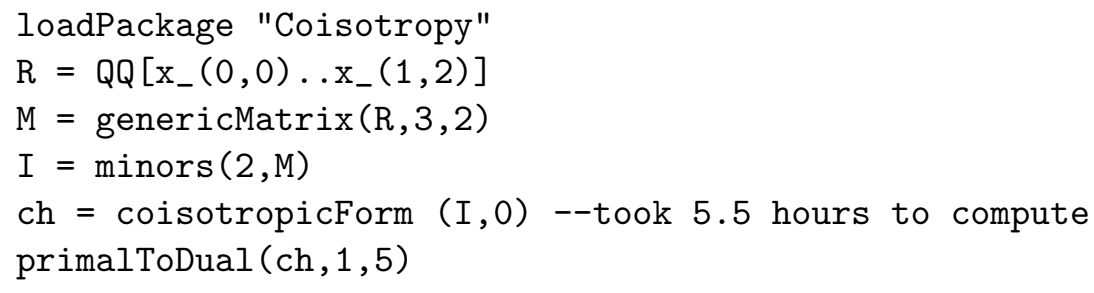

Code D. The following code verifies that the incidence variety of inflectional lines of a random cubic surface as described in Remark 5.44 is smooth. We compute the ideal I of the incidence variety in the same affine chart as in Theorem 5.37

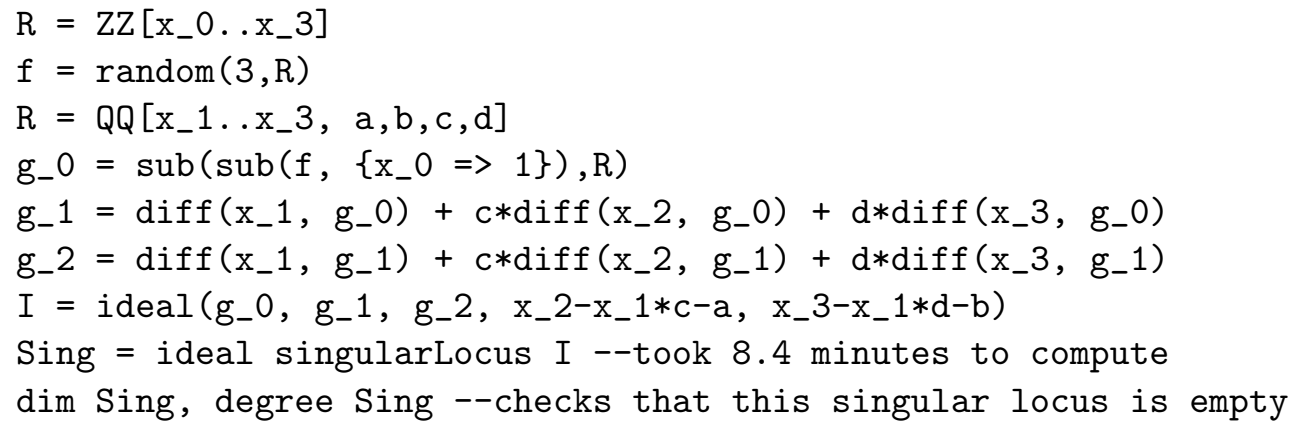

Code E. We use the package Coisotropy (see Section 3.9) to compute the dual variety of the surface $\mathcal{S}$ defined in Example 6.3. 


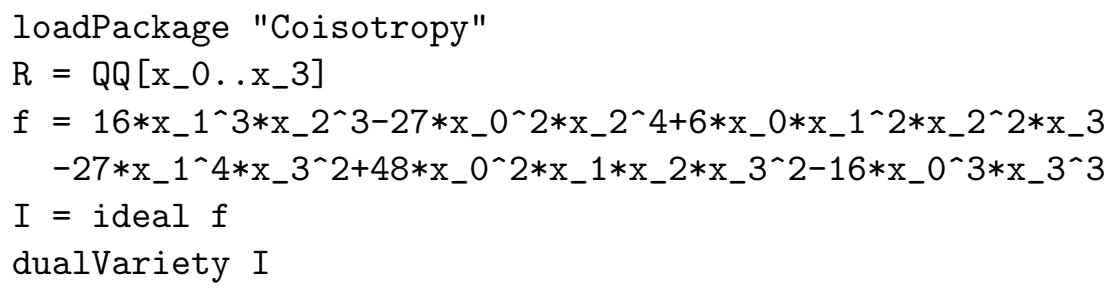

Now we compute the curve $C \subset \operatorname{Gr}\left(1, \mathbb{P}^{3}\right)$ which encodes the ruling of $\mathcal{S}$.

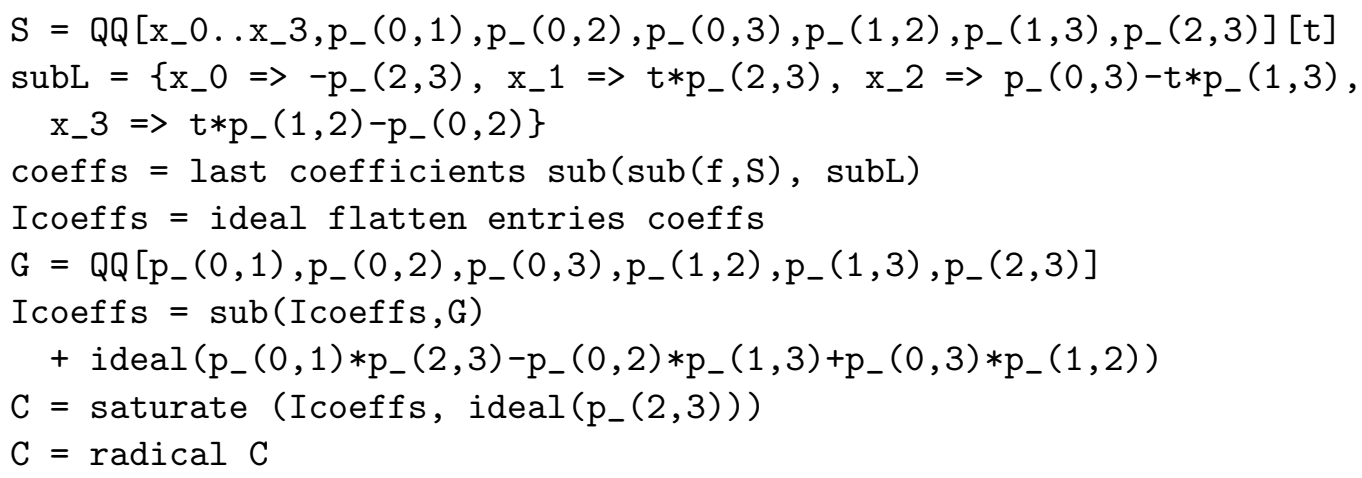

Finally, we compute the edge of regression of $\mathcal{S}$. We do this by first computing the union of the lines which are dual to the lines on $C$. This is the dual surface of the edge of regression; see Figure 6.1.

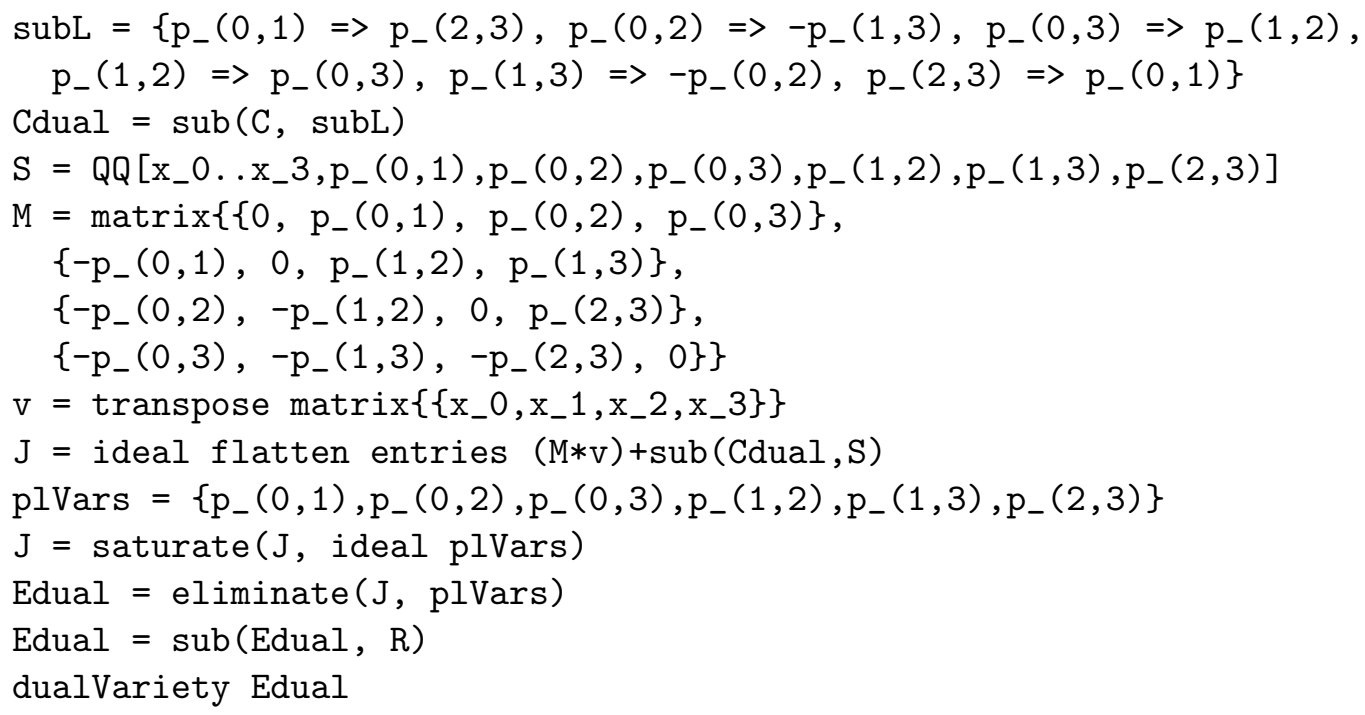

Code F. We compute the tangential surface of the curve $X$ in Example6.11.

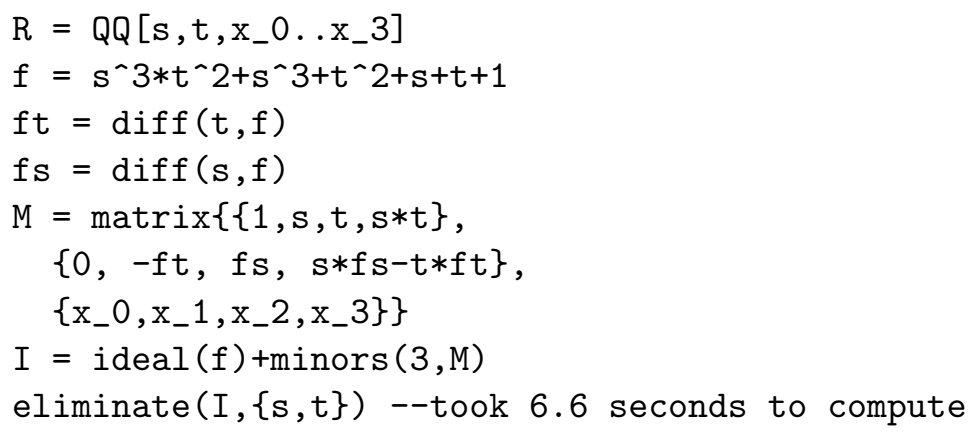


Code G. We compute the dual curve of the edge surface of the curve $X$ as in Example 6.12. First, we need a function which computes the $\mathrm{k}$-th subresultant of two polynomials $p$ and $q$ in one variable $t$.

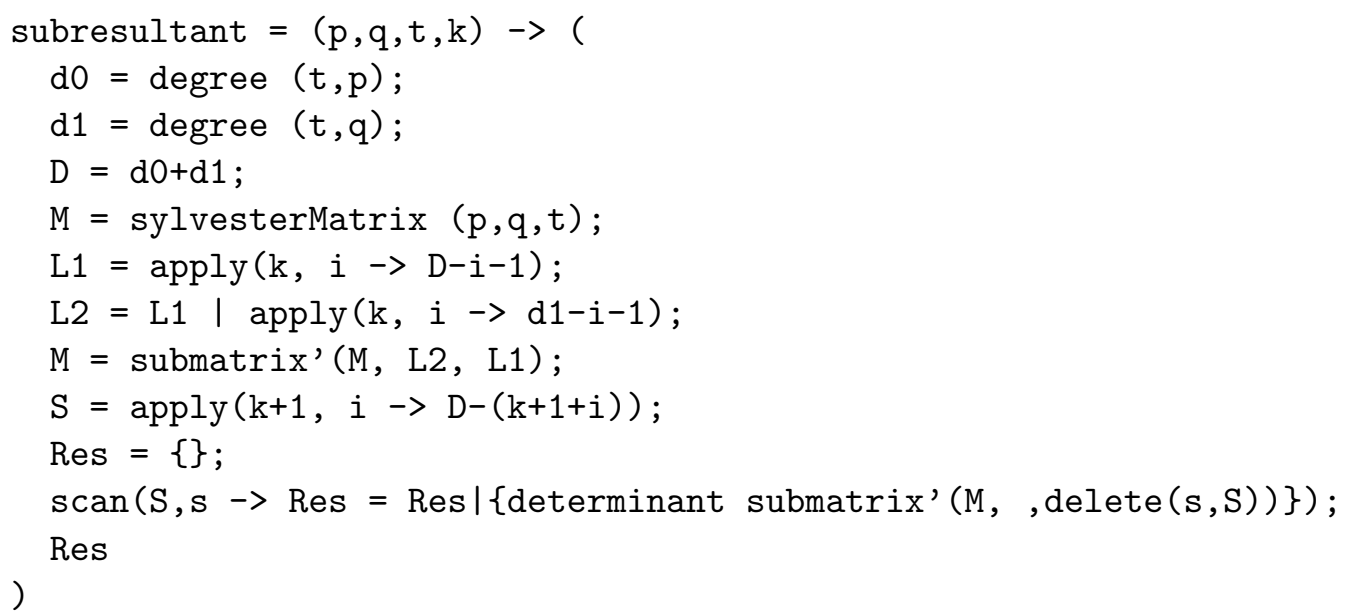

Secondly, we precompute $\Delta_{(2,2)}(5)$.

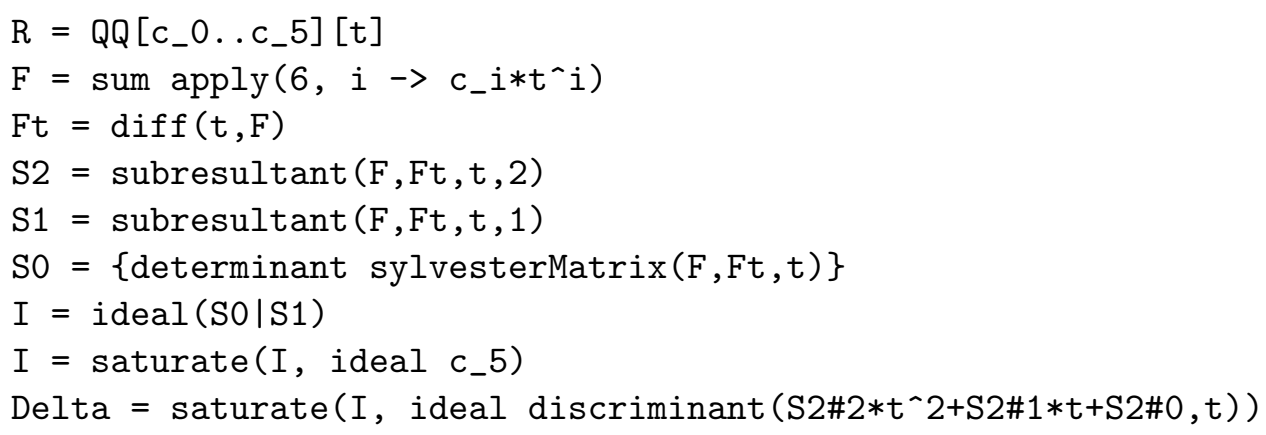

Finally, we compute $\mathcal{E}^{p}(X)$.

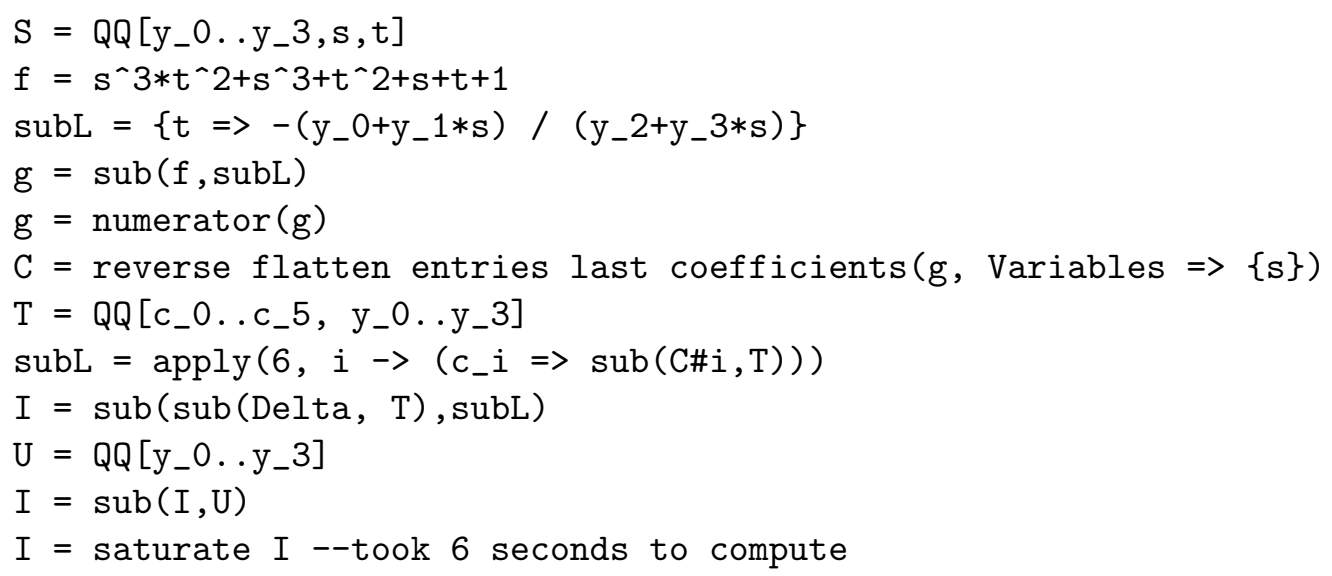

Code H. The following code illustrates Proposition 6.14 for rational curves of degree six.

$\mathrm{A}=\operatorname{random}\left(\mathrm{ZZ} \wedge 4, \mathrm{ZZ}^{\wedge} 7\right)$

Ext2A = exteriorPower $(2, A)$ 


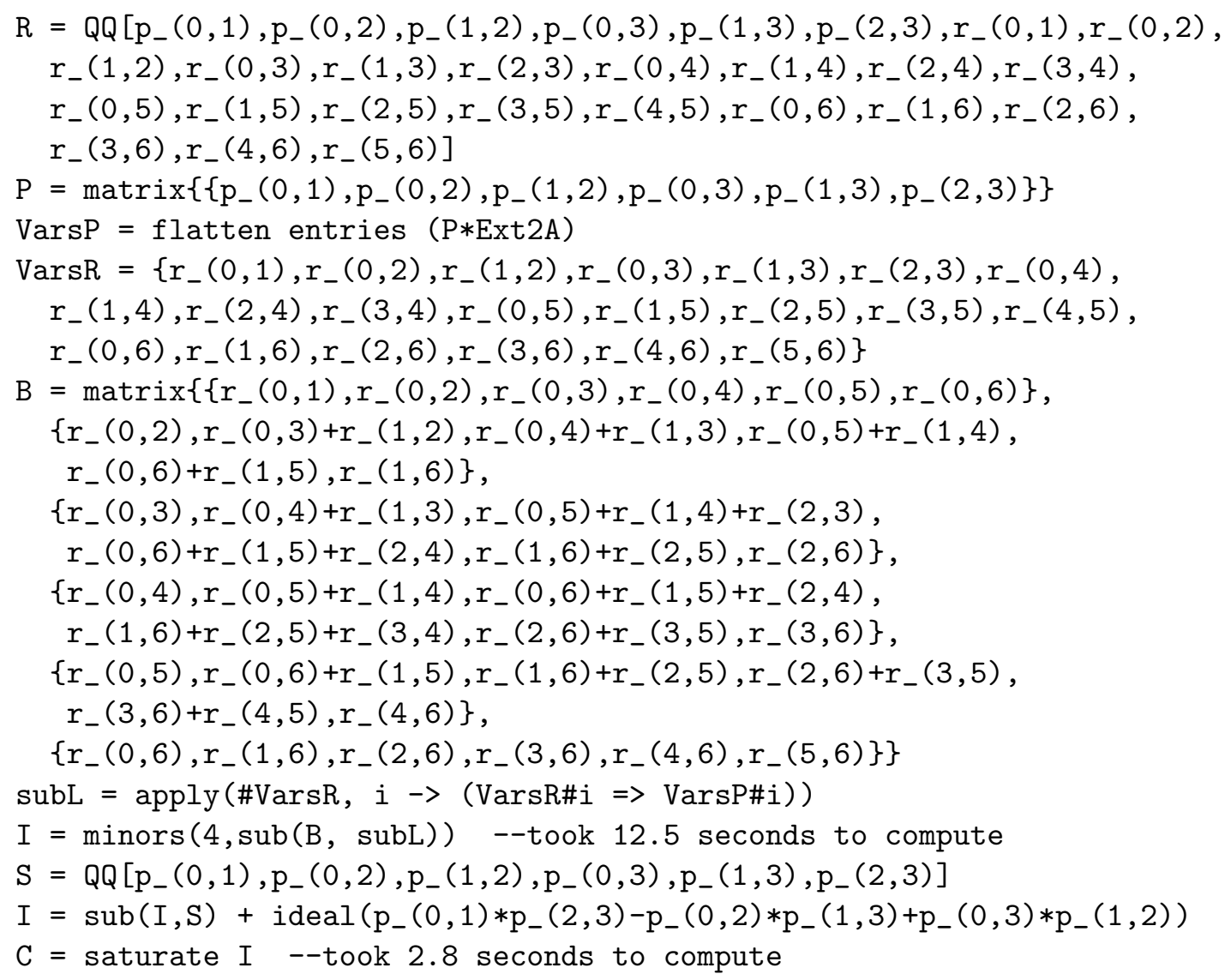

Code I. We compute the trisecant surface of the curve $X$ in Example 6.15. First, we need a function which computes a polynomial in Plücker coordinates from a given polynomial in Stiefel coordinates, if possible. The following is an implementation by Paolo Tripoli of [99, Algorithm 3.2.8].

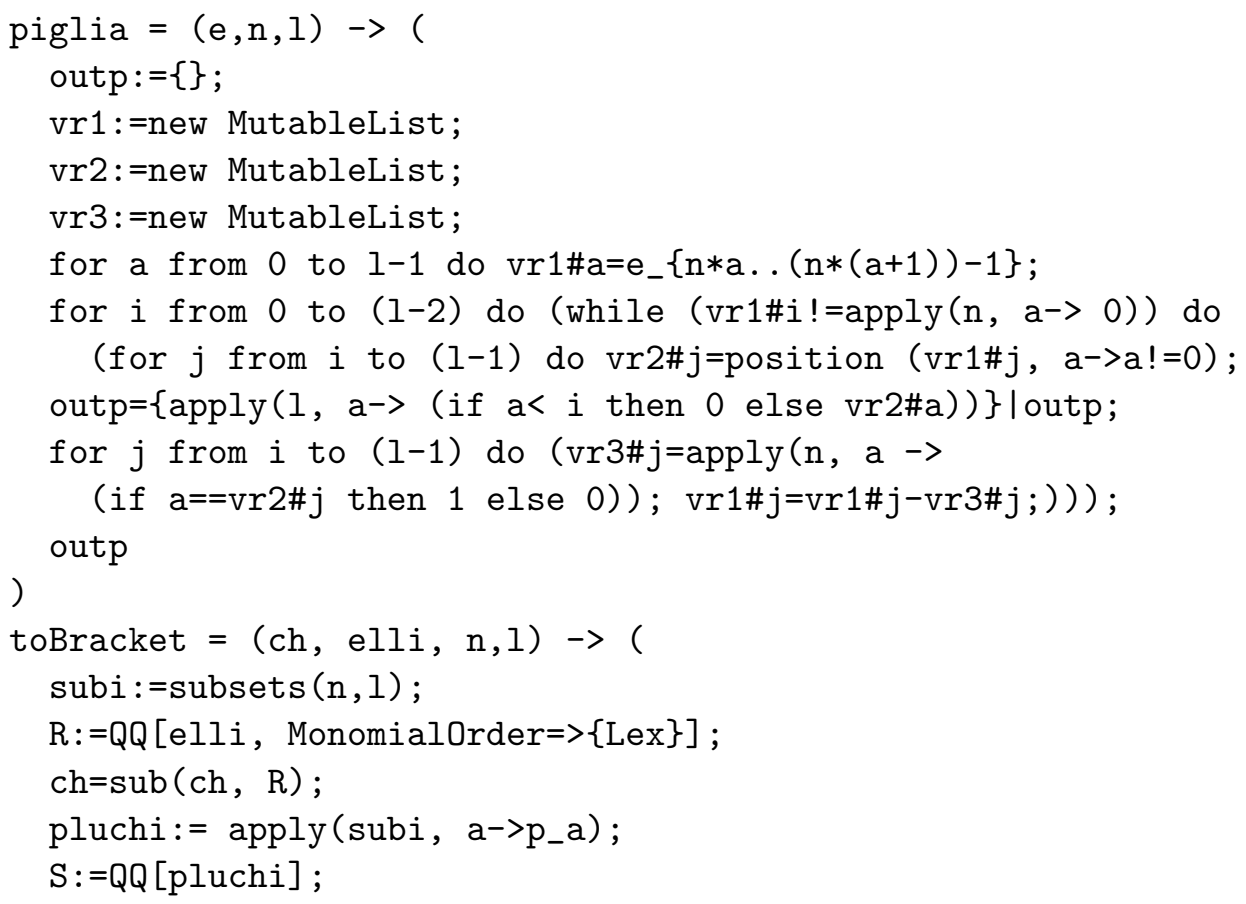




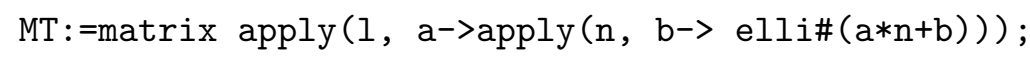

$\mathrm{E}:=\operatorname{apply}($ subi, a-> det MT_a);

$E=\operatorname{apply}(E, a->\operatorname{sub}(a, R))$;

OUT $:=0 \_S$;

while $(\mathrm{ch} !=0)$ do $\{$ lead:=leadMonomial (ch);

coef : =leadCoefficient (ch);

$\mathrm{b}:=$ piglia (flatten exponents lead, $\mathrm{n}, \mathrm{l})$;

OUT $=$ OUT + sub (coef, $S) *\left(\right.$ product apply $\left.\left(b, a->p_{-} a\right)\right)$;

$\mathrm{ch}=\mathrm{ch}-\operatorname{coef} *($ product $\operatorname{apply}(\mathrm{b}, \mathrm{c}->\mathrm{E} \#(\operatorname{position}($ subi, $\mathrm{a}->\mathrm{a}==\mathrm{c})))) ;\}$;

OUT

)

Secondly, we compute the prime ideal of $\mathcal{D}^{\ell}(X)$.

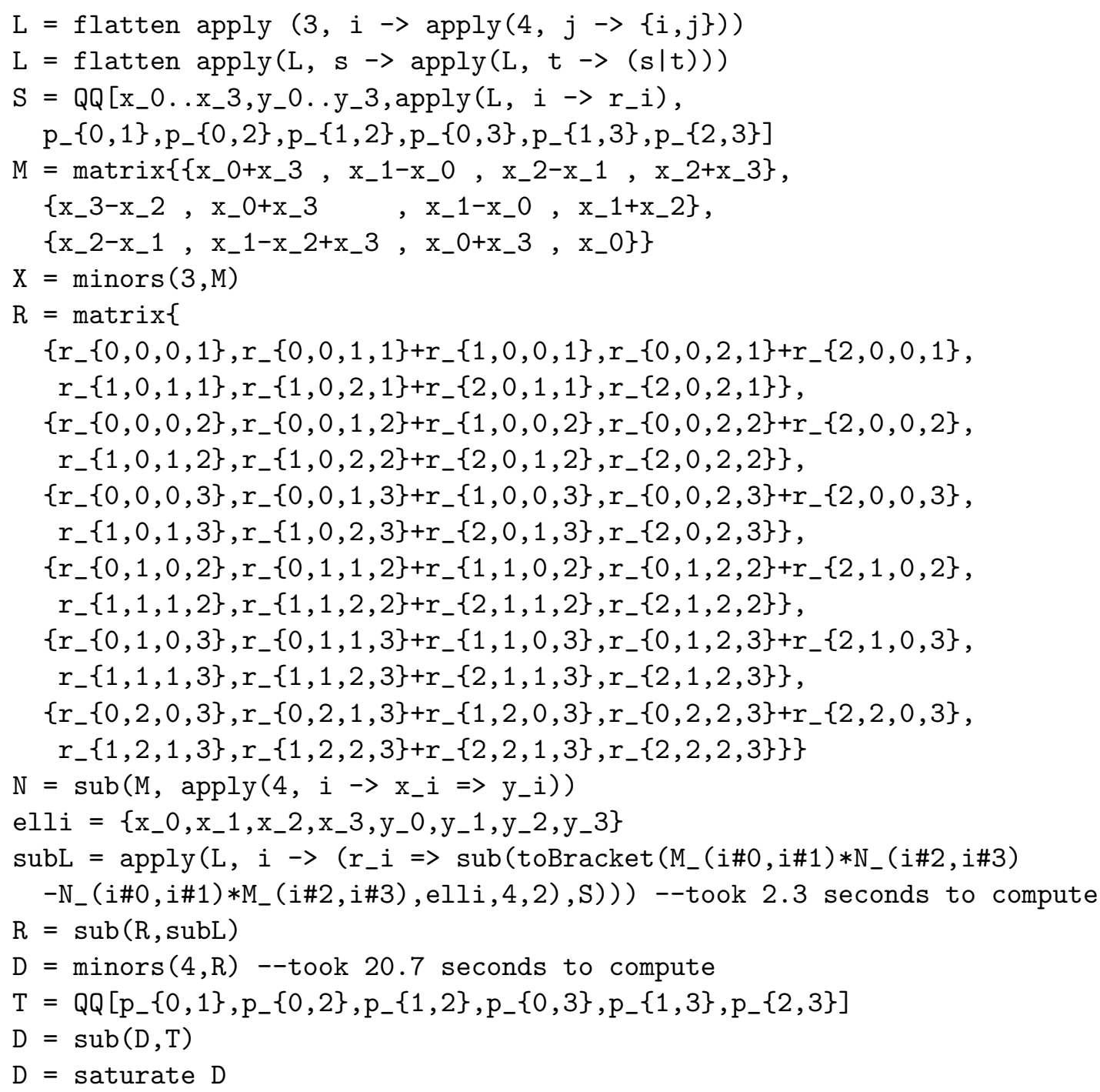

Finally, we compute the defining polynomial of the trisecant surface of $X$.

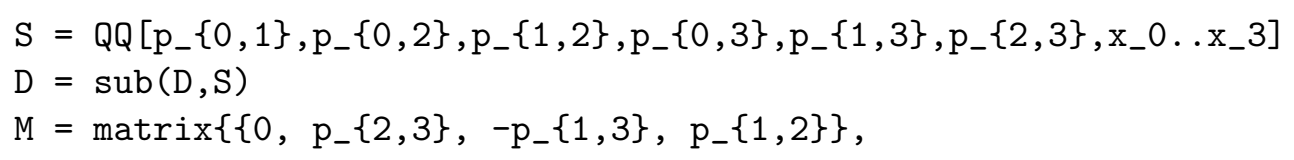




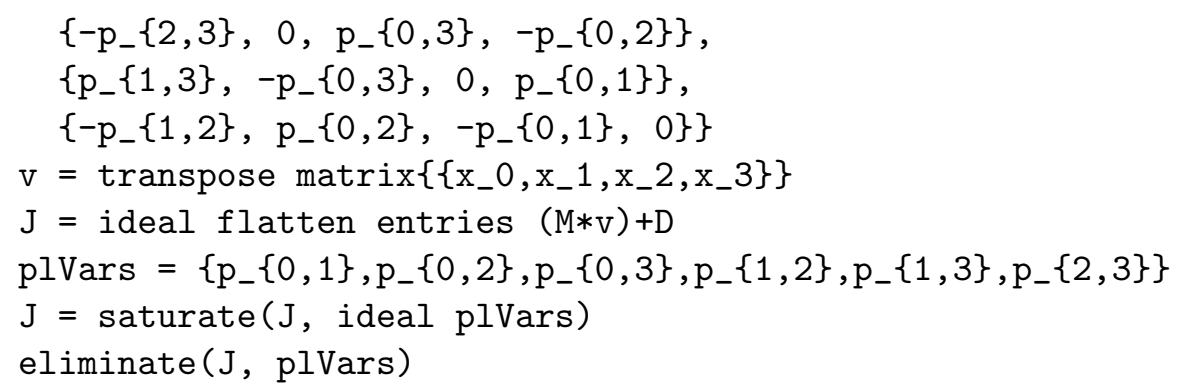

Code J. We compute the tangential surface of the curve $X$ in Example 6.16.

$\mathrm{R}=\mathrm{QQ}\left[\mathrm{x}_{-} 0 \ldots \mathrm{x}_{-} 3, \mathrm{y}_{-} 0 \ldots \mathrm{y}_{-} 3\right]$

$X=i d e a l\left(\operatorname{sum} \operatorname{apply}\left(4, i \rightarrow y_{-} i^{\wedge} 2\right)\right.$, sum apply(4, $\left.i \rightarrow y_{-} i^{\wedge} 3\right)$ )

$\mathrm{v}=$ transpose matrix $\left\{\left\{0,0,0,0, \mathrm{x}_{-} 0, \mathrm{x}_{-} 1, \mathrm{x}_{-} 2, \mathrm{x}_{-} 3\right\}\right\}$

Inc $=\mathrm{X}+$ ideal flatten entries ( (transpose jacobian $\mathrm{X}) * \mathrm{v})$

Inc $=$ saturate $\left(\right.$ Inc, ideal $\left.\mathrm{y}_{-} 0\right)$--took 11.8 minutes to compute

eliminate(Inc, \{y_0,y_1,y_2,y_3\}) --took 51.9 seconds to compute

Since saturating Inc by the ideal generated by $y_{0}, y_{1}, y_{2}, y_{3}$ took too long, we instead tried to compute the saturation above, which also yields the desired result after elimination.

Moreover, we describe how to compute the trisecant surface of $X$. For this, we first compute the corresponding curve $\mathcal{D}^{\ell}(X)$ in $\operatorname{Gr}\left(1, \mathbb{P}^{3}\right)$ with subresultants. We use the function subresultant in Code $\mathrm{G}$.

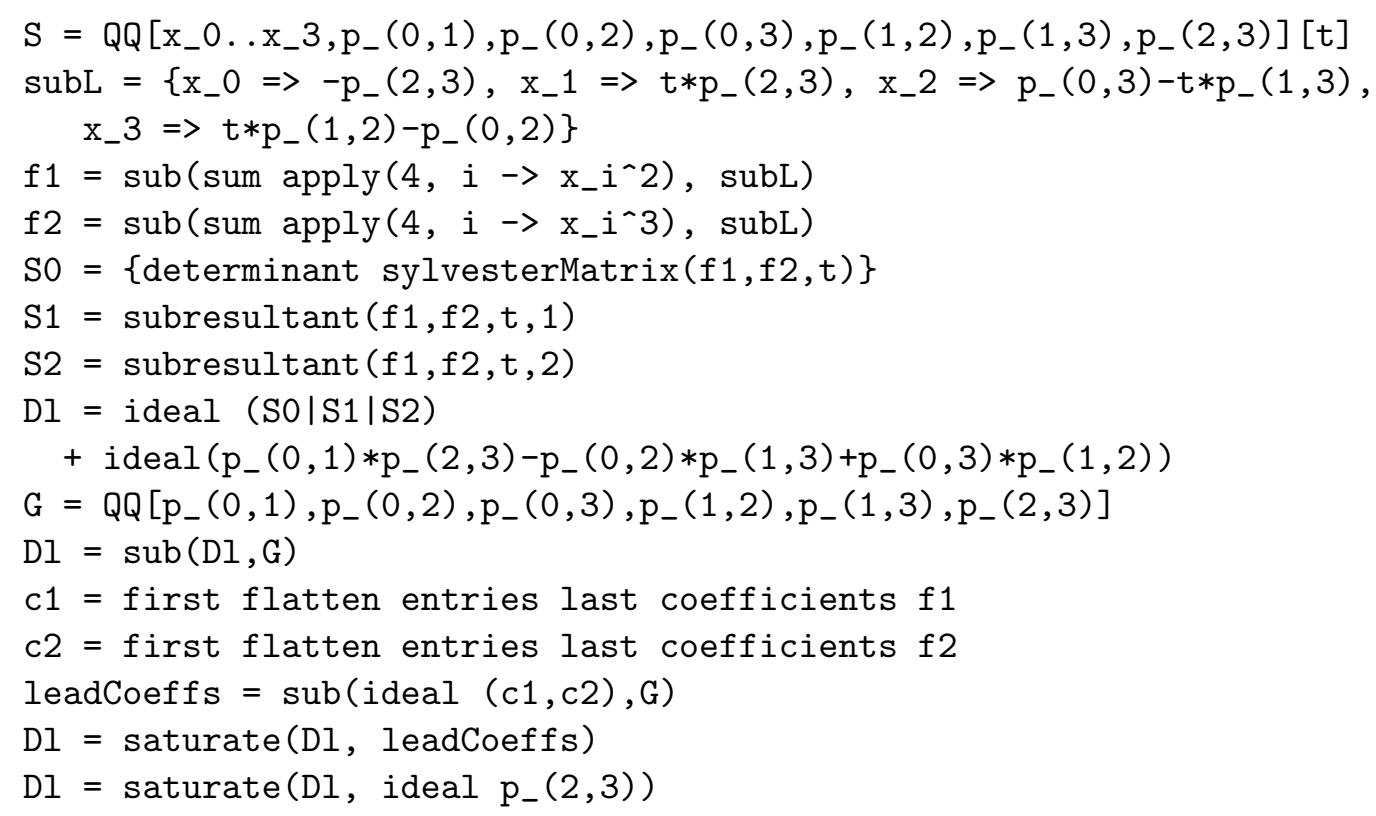

It turns out that $\mathcal{D}^{\ell}(X)$ has two quadratic components, which are both rulings of the quadric surface $\mathcal{D}(X)$ :

DI1 = first decompose D1

$\mathrm{D} 12$ = last decompose $\mathrm{D} 1$

$\mathrm{S}=\mathrm{QQ}\left[\mathrm{x}_{-} 0 \ldots \mathrm{x}_{-} 3, \mathrm{p}_{-}(0,1), \mathrm{p}_{-}(0,2), \mathrm{p}_{-}(0,3), \mathrm{p}_{-}(1,2), \mathrm{p}_{-}(1,3), \mathrm{p}_{-}(2,3)\right]$

$M=\operatorname{matrix}\left\{\left\{0, p_{-}(0,1), p_{-}(0,2), p_{-}(0,3)\right\}\right.$, 


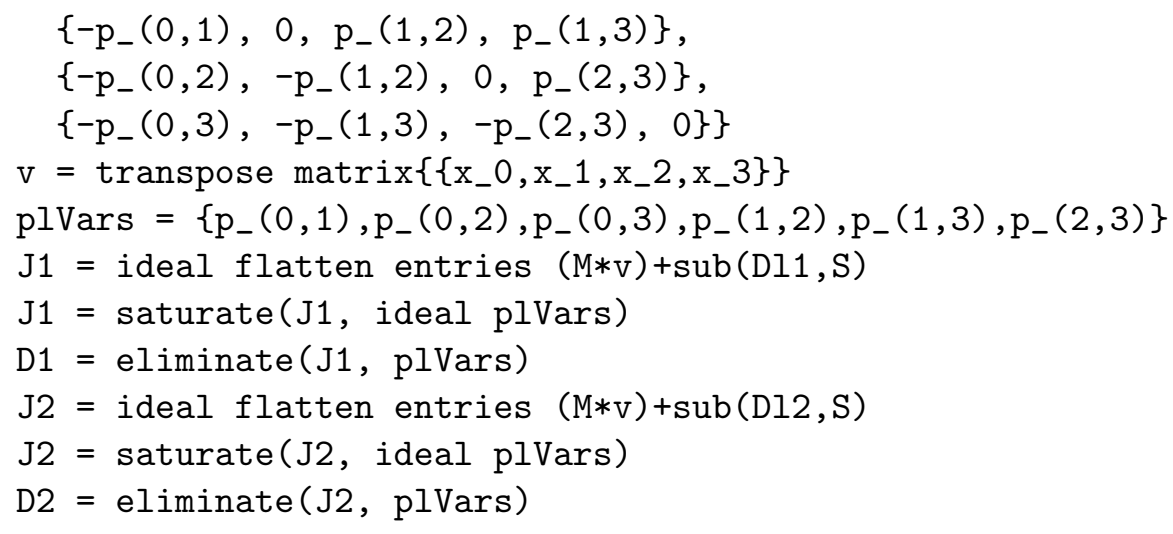

Code K. In the following, we use the function subresultant in Code G. First, we compute the multiple root locus $\Delta_{(3)}(3)$.

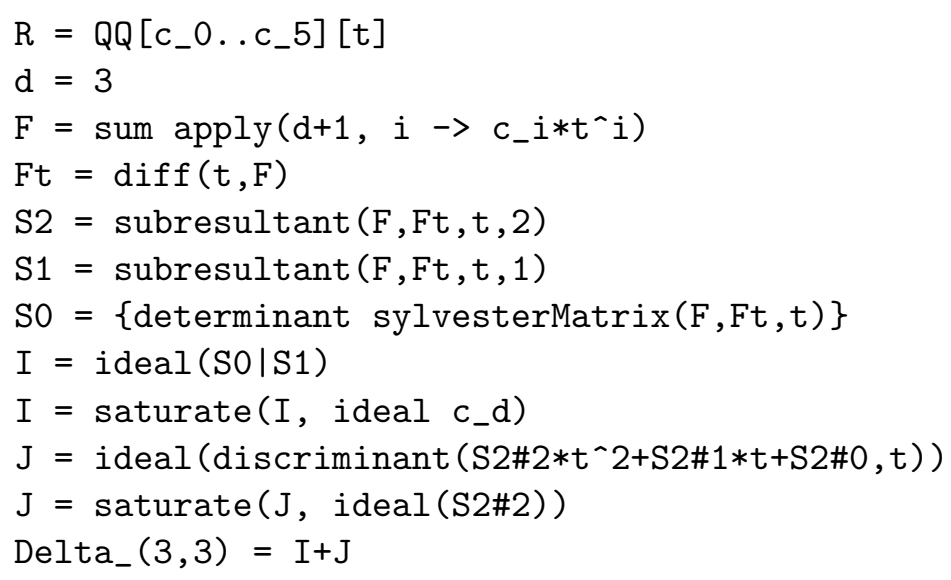

Secondly, we compute $\Delta_{(4)}(4)$; see Example 6.25

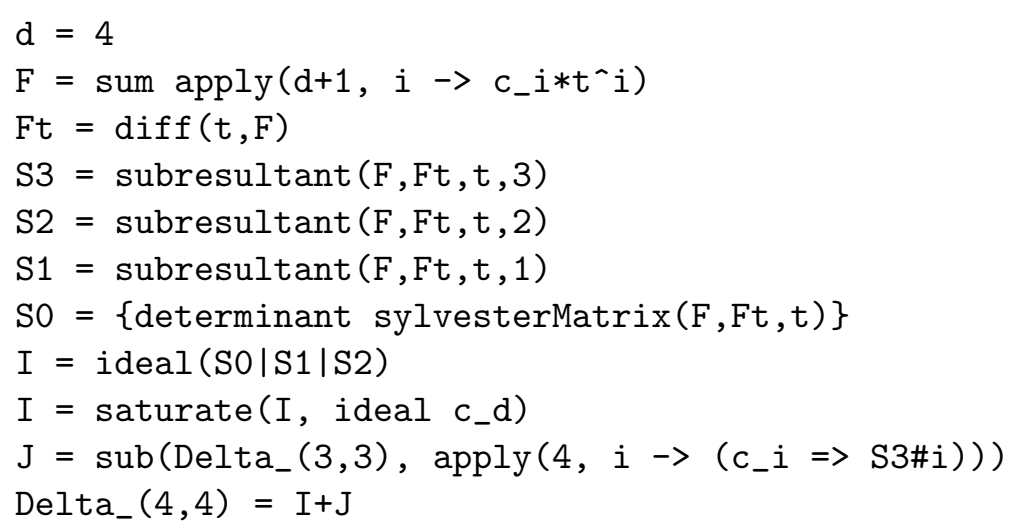

Finally, we compute $\Delta_{(4)}(5)$; see again Example 6.25.

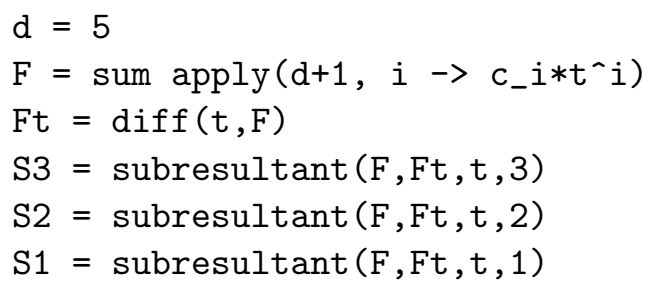




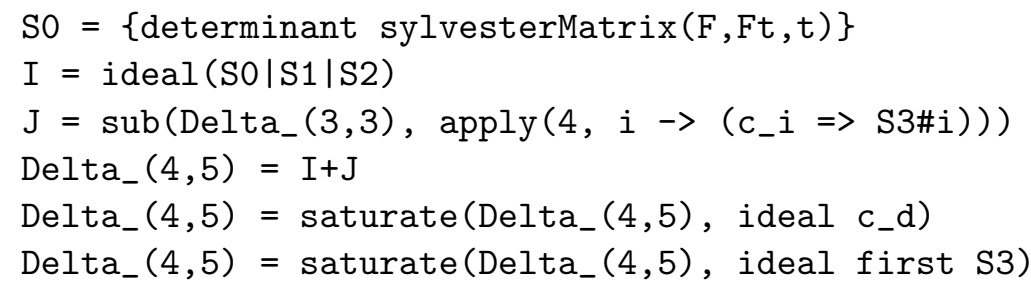

Code L. We compute the curve $\mathcal{F}^{\ell}(X)$ in $\operatorname{Gr}\left(1, \mathbb{P}^{3}\right)$ corresponding to the flecnodal surface of the surface $X$ in Example 6.26

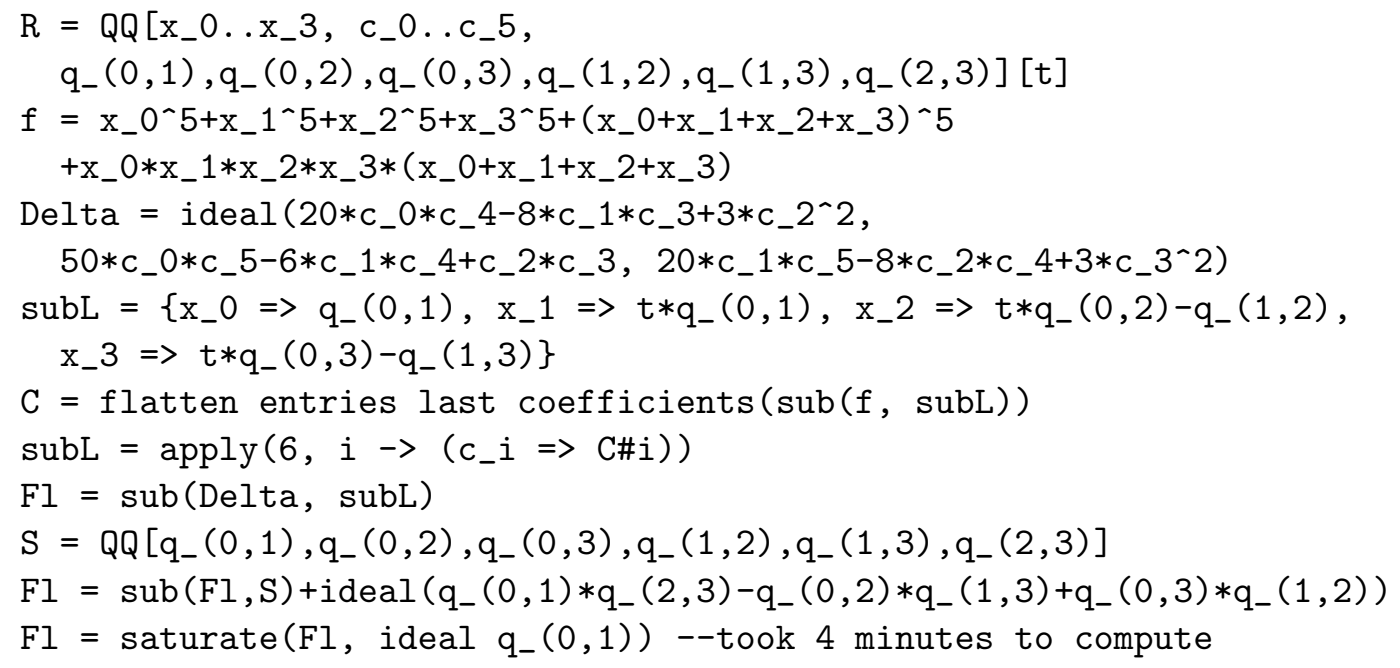

Code M. The following computes the parabolic surface of the Fermat cubic surface $X$ as in Example 6.29. We start by computing the corresponding curve $\mathcal{P}^{\ell}(X)$ in $\operatorname{Gr}\left(1, \mathbb{P}^{3}\right)$.

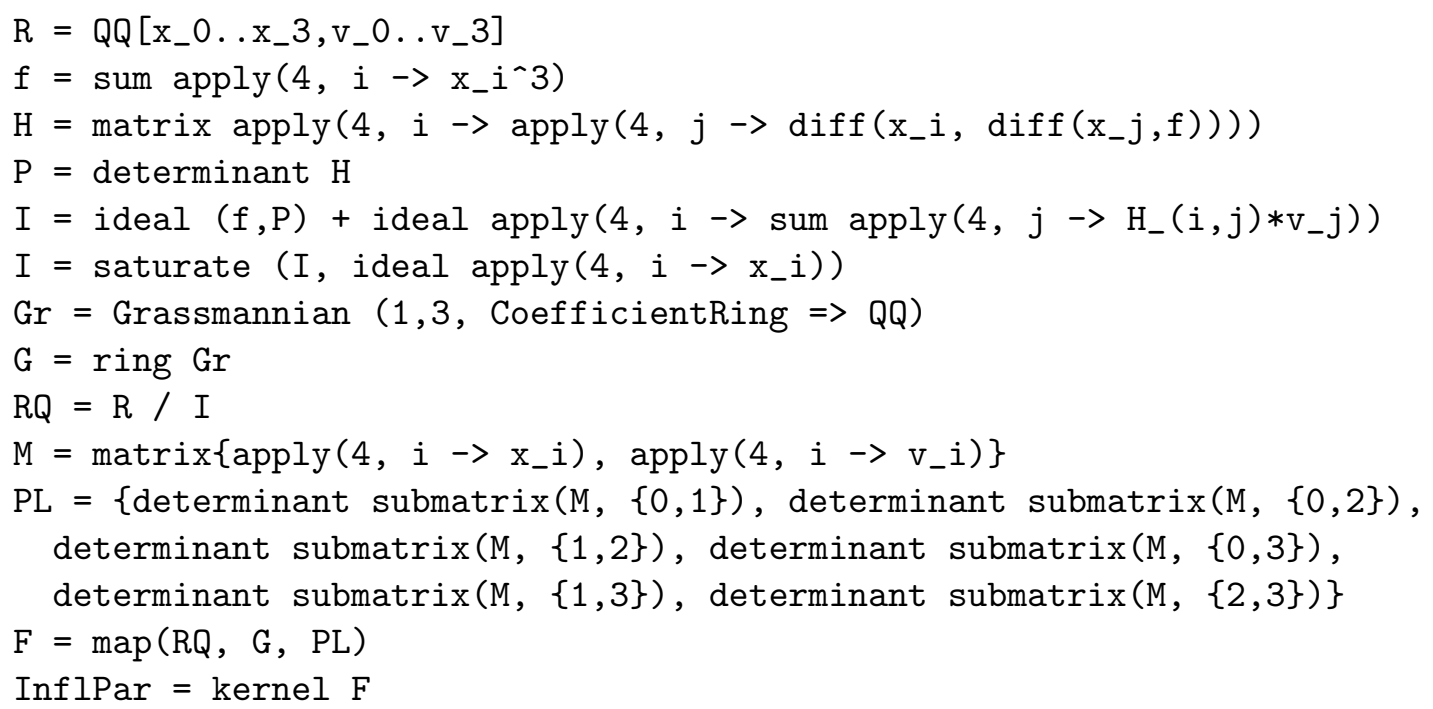

From this, we compute the defining equation of the parabolic surface and its factorization. When we have to saturate by the ideal generated by the Plücker variables, we instead compute iterated colon ideals due to run time efficiency.

$R=Q Q\left[v_{-} 0 \ldots v_{-} 3, p_{-}(0,1), p_{-}(0,2), p_{-}(0,3), p_{-}(1,2), p_{-}(1,3), p_{-}(2,3)\right]$ 


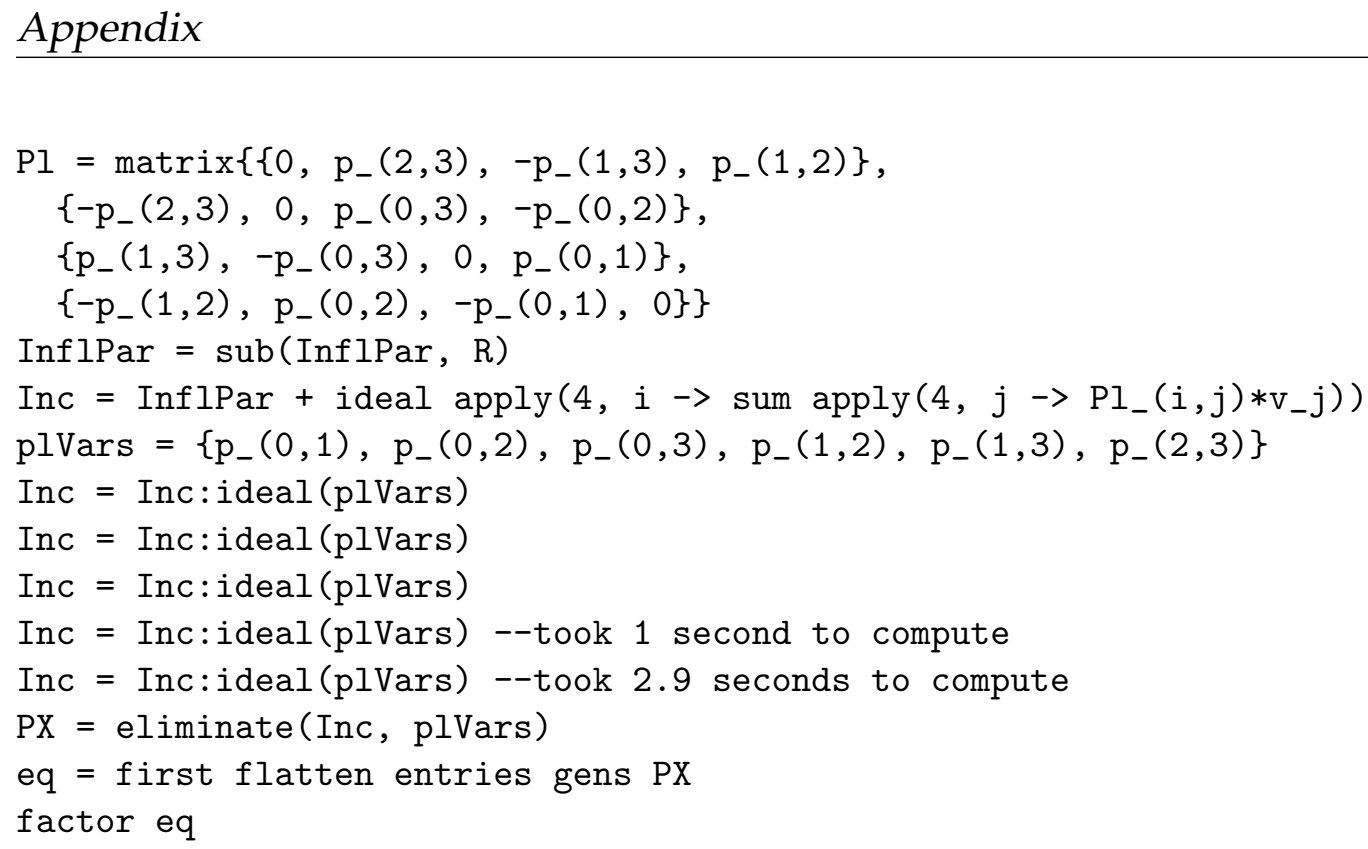

Code $\mathbf{N}$. We compute the curve $\mathcal{P}^{\ell}(X)$ in $\operatorname{Gr}\left(1, \mathbb{P}^{3}\right)$ corresponding to the parabolic surface of the cubic surface $X$ in Example 6.30. For this, we use the same code as in the first half of Code $M$; we only have to exchange $f$ by

$f=\operatorname{sum} \operatorname{apply}\left(4, i \rightarrow x_{-} i^{\wedge} 3\right)+\left(x_{-} 0+2 * x_{-} 1+3 * x_{-} 2+4 * x_{-} 3\right) \wedge 3$

This time InflPar = kernel $\mathrm{F}$ took 11.3 seconds. With this computation, we are able to stab the parabolic surface of $X$ with a given line. We use the line in Example 6.30.

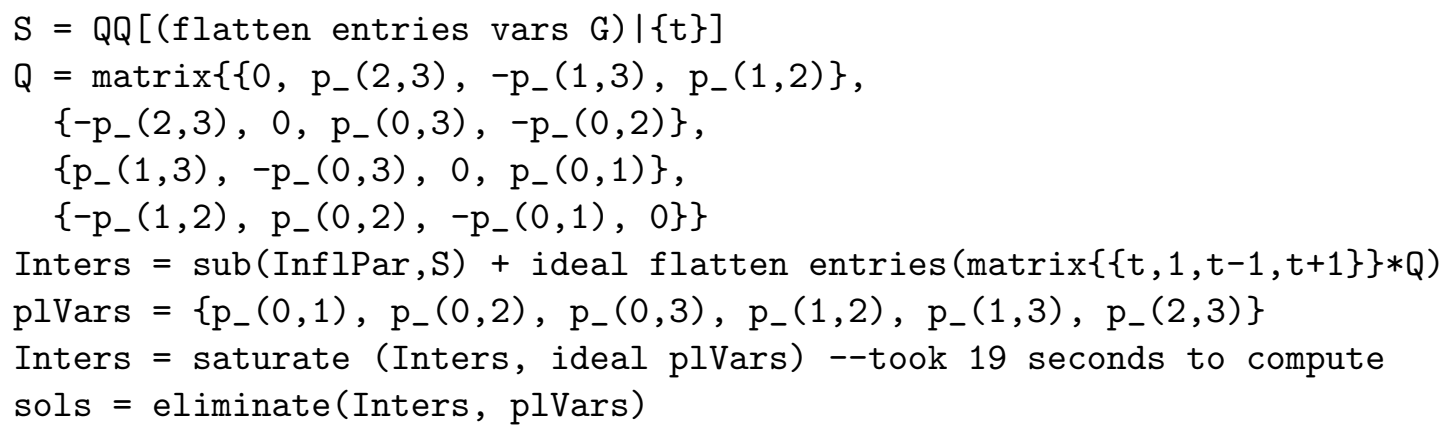

The ideal sols is generated by the univariate polynomial of degree 30 given in Example 6.30. One can solve for its real roots with a variety of software, e.g., with the command realroots of the computer algebra system Maxima.

Now we compute this polynomial of degree 30 by first computing the dual curve of the parabolic surface of $X$.

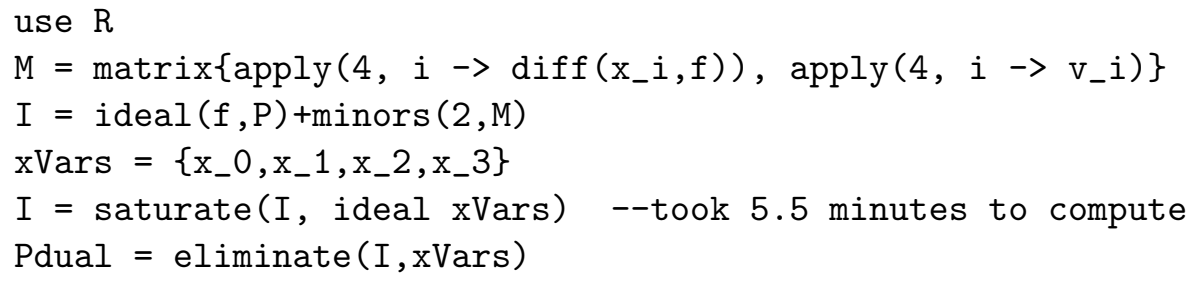

Finally, we compute the univariate polynomial of degree 30 as described at the end of Example 6.30. 


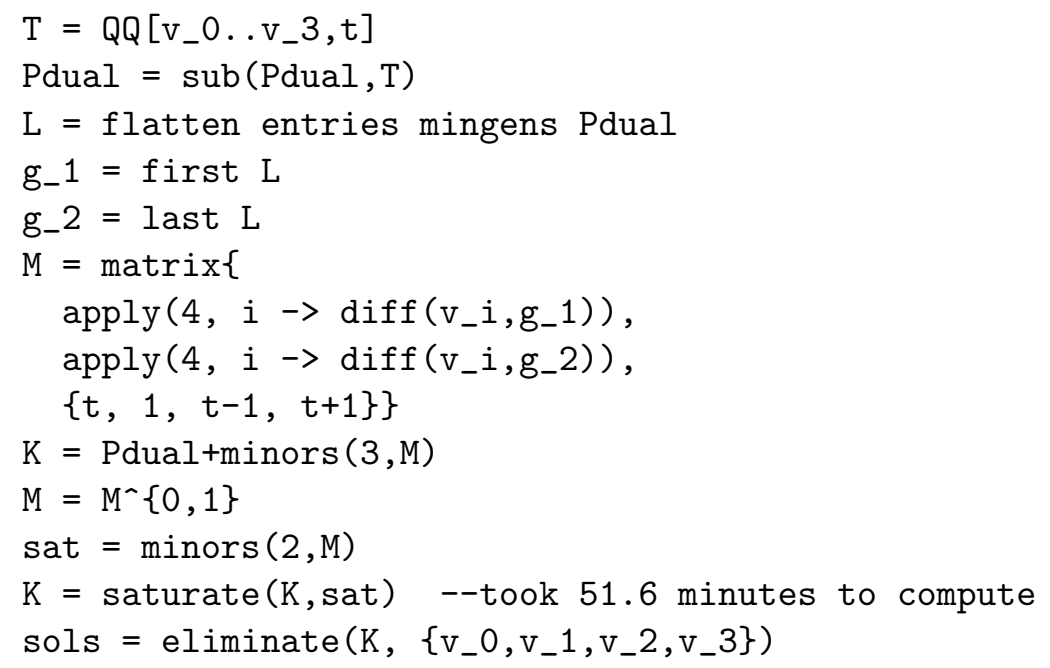





\section{Bibliography}

[1] Jounaidi Abdeljaoued, Gema M. Diaz-Toca, and Laureano GonzalezVega. Minors of Bezout Matrices, Subresultants and the Parameterization of the Degree of the Polynomial Greatest Common Divisor. International Journal of Computer Mathematics, 81(10):1223-1238, 2004.

[2] Carlos Améndola, Kathlén Kohn, Sara Lamboglia, Diane Maclagan, Ben Smith, Jeff Sommars, Paolo Tripoli, and Magdalena Zajaczkowska. Computing Tropical Varieties in Macaulay2. arXiv: 1710.10651, 2017.

[3] Vladimir Igorevich Arnol'd. Singularities of Smooth Mappings. Russian Mathematical Surveys, 23:1-43, 1968.

[4] Enrique Arrondo. Subvarieties of Grassmannians. In Lecture Note Series, volume 10. Dipartimento di Matematica Univ. Trento, 1996. Available at www. mat.ucm.es/ ${ }^{\sim}$ arrondo/trento.pdf.

[5] Enrique Arrondo, Marina Bertolini, and Cristina Turrini. A Focus on Focal Surfaces. Asian J. Math., 5:535-560, 2001.

[6] Jon Barwise and Paul C. Eklof. Lefschetz's Principle. Journal of Algebra, 13(4):554-570, 1969.

[7] M.C. Beltrametti, E. Carletti, G. Monti Bragadin, and D. Gallarati. Lectures on Curves, Surfaces and Projective Varieties: A Classical View of Algebraic Geometry, volume 9 of EMS Textbooks in Mathematics. European Mathematical Society, 2009.

[8] Marie-Amélie Bertin. On the Singularities of the Trisecant Surface to a Space Curve. Le Matematiche, 53(3):15-22, 1999.

[9] Tristram Bogart, Anders N. Jensen, David Speyer, Bernd Sturmfels, and Rekha R. Thomas. Computing Tropical Varieties. Journal of Symbolic Computation, 42:54-73, 2007.

[10] Kevin W. Bowyer and Charles R. Dyer. Aspect Graphs: An Introduction and Survey of Recent Results. International Journal of Imaging Systems and Technology, 2(4):315-328, 1990.

[11] Jarosław Buczyński and Joseph M. Landsberg. Ranks of Tensors and a Generalization of Secant Varieties. Linear Algebra and its Applications, 438(2):668-689, 2013. 
[12] Peter Bürgisser. Condition of Intersecting a Projective Variety with a Varying Linear Subspace. SIAM Journal on Applied Algebra and Geometry, 1(1):111-125, 2017.

[13] Peter Bürgisser, Kathlén Kohn, Pierre Lairez, and Bernd Sturmfels. Computing the Chow Variety of Quadratic Space Curves. In I. Kotsireas, S. Rump, and C. Yap, editors, Mathematical Aspects of Computer and Information Sciences, MACIS 2015, Berlin, pages 130-136, 2016.

[14] Peter Bürgisser and Antonio Lerario. Probabilistic Schubert Calculus. arXiv:1612.06893, 2016.

[15] Enrico Carlini and Jaydeep V. Chipalkatti. On Waring's Problem for Several Algebraic Forms. Commentarii Mathematici Helvetici, 78(3):494-517, 2003.

[16] Fabrizio M.E. Catanese. Cayley Forms and Self-Dual Varieties. In Proceedings of the Edinburgh Mathematical Society (Series 2), volume 57, pages 89-109. Cambridge University Press, 2014.

[17] Arthur Cayley. On the Singularities of Surfaces. The Cambridge and Dublin Mathematical Journal, 7:166-171, 1852.

[18] Arthur Cayley. On a New Analytical Representation of Curves in Space. The Quarterly Journal of Pure and Applied Mathematics, 3:225-236, 1860.

[19] Lin Chen and Dragomir Ž Đoković. Properties and Construction of Extreme Bipartite States Having Positive Partial Transpose. Communications in Mathematical Physics, 323(1):241-284, 2013.

[20] Luca Chiantini and Marc Coppens. Grassmannians of Secant Varieties. In Forum Mathematicum, volume 13, pages 615-628. de Gruyter, 2001.

[21] Jaydeep V. Chipalkatti. The Waring Locus of Binary Forms. Communications in Algebra, 32(4):1425-1444, 2004.

[22] Jaydeep V. Chipalkatti and Anthony V. Geramita. On Parameter Spaces for Artin Level Algebras. The Michigan Mathematical Journal, 51:187-208, 2003.

[23] Wei-Liang Chow and Bartel L. van der Waerden. Zur algebraischen Geometrie IX. Über zugeordnete Formen und algebraische Systeme von algebraischen Mannigfaltigkeiten. Mathematische Annalen, 113:692-704, 1937.

[24] Ciro Ciliberto and Filip Cools. On Grassmann Secant Extremal Varieties. Advances in Geometry, 8(3):377-386, 2008. 
[25] Susan Jane Colley. Lines Having Specified Contact with Projective Varieties. In Proceedings of the 1984 Vancouver Conference in Algebraic Geometry, volume 6, pages 47-70, 1986.

[26] Susan Jane Colley. Enumerating Stationary Multiple-Points. Advances in Mathematics, 66(2):149-170, 1987.

[27] Filip Cools. On the Singular Locus of Grassmann Secant Varieties. Bulletin of the Belgian Mathematical Society-Simon Stevin, 16(5):799-803, 2009.

[28] John Dalbec and Bernd Sturmfels. Introduction to Chow Forms. In Invariant Methods in Discrete and Computational Geometry, pages 37-58. Springer, 1995.

[29] Pietro De Poi. Congruences of Lines with One-Dimensional Focal Locus. Portugaliae Mathematica, 61(3):329-338, 2004.

[30] C. DeConcini, M. Goresky, R. MacPherson, and C. Procesi. On the Geometry of Quadrics and Their Degenerations. Commentarii Mathematici Helvetici, 63:337-413, 1988.

[31] Ernst Ulrich Deuker. On the Way to a Grammar of Free Musical Speech: A Pentatonic Approach to Improvisation. Books on Demand, 2016.

[32] Igor V. Dolgachev. Classical Algebraic Geometry: A Modern View. Cambridge University Press, 2012.

[33] William L. Edge. The Theory of Ruled Surfaces. Cambridge University Press, 1931.

[34] David Eisenbud and Joe Harris. 3264 and All That: A Second Course in Algebraic Geometry. Cambridge University Press, 2016.

[35] David Eisenbud, Frank-Olaf Schreyer, and Jerzy Weyman. Resultants and Chow Forms via Exterior Syzygies. Journal of the American Mathematical Society, 16(3):537-579, 2003.

[36] Paul C. Eklof. Lefschetz's Principle and Local Functors. Proceedings of the American Mathematical Society, 37(2):333-339, 1973.

[37] Laura Escobar and Allen Knutson. The Multidegree of the Multi-Image Variety. In G.G. Smith and B. Sturmfels, editors, Combinatorial Algebraic Geometry, volume 80 of Fields Institute Communications, pages 283-296. Springer, 2017.

[38] David Forsyth and Jean Ponce. Computer Vision: A Modern Approach. Pearson, 2 edition, 2012.

[39] Gerhard Frey and Hans-Georg Rück. The Strong Lefschetz Principle in Algebraic Geometry. manuscripta mathematica, 55:385-401, 1986. 
[40] William Fulton. Intersection Theory. Springer, 2 edition, 1998.

[41] William Fulton, Steven L. Kleiman, and Robert MacPherson. About the Enumeration of Contacts. Algebraic Geometry - Open Problems, pages 156196, 1983.

[42] Ewgenij Gawrilow and Michael Joswig. polymake: A framework for analyzing convex polytopes. In Polytopes - Combinatorics and Computation (Oberwolfach, 1997), volume 29 of DMV Sem., pages 43-73. Birkhäuser, 2000.

[43] Israel M. Gel'fand, Mikhail M. Kapranov, and Andrei V. Zelevinsky. Discriminants, Resultants and Multidimensional Determinants, volume 227 of Graduate Texts in Mathematics. Birkhäuser, 1994.

[44] N. Goldstein. The Geometry of Surfaces in the 4-Quadric. Rend. Sem. Mat. Univ. Politec. Torino, 43(3):467-499, 1985.

[45] Daniel R. Grayson and Michael E. Stillman. Macaulay2, a Software System for Research in Algebraic Geometry. Available at http://www . math . uiuc. edu/Macaulay2/.

[46] Mark L. Green and Ian Morrison. The Equations Defining Chow Varieties. Duke Mathematical Journal, 53:733-747, 1986.

[47] Phillip Griffiths and Joe Harris. Principles of Algebraic Geometry. John Wiley \& Sons, 1978.

[48] Simon Hampe. A-Tint: A polymake Extension for Algorithmic Tropical Intersection Theory. European J. Combin., 36:579-607, 2014.

[49] Joe Harris. Algebraic Geometry: A First Course, volume 133 of Graduate Texts in Mathematics. Springer, 1992.

[50] Robin Hartshorne. Algebraic Geometry, volume 52 of Graduate Texts in Mathematics. Springer, 1977.

[51] Allen Hatcher. Algebraic Topology. Cambridge University Press, 2002.

[52] Andrew Hoefel and Michael Stillman. Interface to Anders Jensen's Gfan Software. A Macaulay2 package.

[53] Audun Holme. The Geometric and Numerical Properties of Duality in Projective Algebraic Geometry. manuscripta mathematica, 61:145-162, 1988.

[54] Anders N. Jensen. Gfan, a Software System for Gröbner Fans and Tropical Varieties. Available at http://home.imf .au.dk/jensen/software/gfan/gfan.html.

[55] Charles Minshall Jessop. A Treatise on the Line Complex. Cambridge University Press, 1903. 
[56] Pal H. Johansen. The Geometry of the Tangent Developable. In Computational Methods for Algebraic Spline Surfaces, pages 95-106. Springer, 2005.

[57] Kent W. Johnson. Immersion and Embedding of Projective Varieties. Acta Mathematica, 140:49-74, 1978.

[58] Camille Jordan. Essai sur la géométrie à $n$ dimensions. Bulletin de la Société Mathématique de France, 3:103-174, 1875.

[59] Yannick L. Kergosien. La famille des projections orthogonales d'une surface et ses singularités. Comptes Rendus Acad. Sc. Paris Sér.I Math, 292:929932, 1981.

[60] Steven L Kleiman. The Transversality of a General Translate. Compositio Math, 28:287-297, 1974.

[61] Steven L. Kleiman. Tangency and Duality. In Proceedings of the 1984 Vancouver Conference in Algebraic Geometry, volume 6, pages 163-225, 1986.

[62] Jan J. Koenderink. Solid Shape. MIT Press, 1990.

[63] Jan J. Koenderink and Andrea J. van Doorn. The Singularities of the Visual Mapping. Biological Cybernetics, 24:51-59, 1976.

[64] Kathlén Kohn. Coisotropic Hypersurfaces in Grassmannians. arXiv:1607.05932, 2016.

[65] Kathlén Kohn and Ernst U. Deuker. Der Komplex der nichtchromatischen Skalen. Mitteilungen der DMV, 25:17-25, 2017. English version The Complex of Non-Chromatic Scales available at arXiv: 1710.05979.

[66] Kathlén Kohn, Bernt Ivar Utstøl Nødland, and Paolo Tripoli. Secants, Bitangents, and Their Congruences. In G.G. Smith and B. Sturmfels, editors, Combinatorial Algebraic Geometry, volume 80 of Fields Institute Communications, pages 87-112. Springer, 2017.

[67] Kathlén Kohn, Bernd Sturmfels, and Matthew Trager. Changing Views on Curves and Surfaces. Acta Mathematica Vietnamica, 43:1-29, 2018.

[68] Dimitry Kozlov. Combinatorial Algebraic Topology, volume 21 of Algorithms and Computation in Mathematics. Springer, 2008.

[69] Ernst Eduard Kummer. Über die algebraischen Strahlensysteme, in's besondere über die der ersten und zweiten Ordnung. Abhandlungen der Königlichen Akademie der Wissenschaften zu Berlin, 1866.

[70] Simon Kurmann. Some Remarks on Equations Defining Coincident Root Loci. Journal of Algebra, 352:223-231, 2012.

[71] Hwangrae Lee and Bernd Sturmfels. Duality of Multiple Root Loci. Journal of Algebra, 446:499-526, 2016. 
[72] Solomon Lefschetz. Algebraic Geometry. Dover Publications, 2005.

[73] Mark Levine. The Jazz Theory Book. Sher Music Co, U.S., 1995.

[74] Diane Maclagan and Bernd Sturmfels. Introduction to Tropical Geometry, volume 161 of Graduate Studies in Mathematics. American Mathematical Society, 2015.

[75] David Mumford. The Red Book of Varieties and Schemes. Number 1358 in Lecture Notes in Mathematics. Springer, 1999.

[76] Sung-Il Pae and Jean Ponce. On Computing Structural Changes in Evolving Surfaces and Their Appearance. International Journal of Computer Vision, 43(2):113-131, 2001.

[77] Sylvain Petitjean. The Complexity and Enumerative Geometry of Aspect Graphs of Smooth Surfaces. In Algorithms in Algebraic Geometry and Applications, volume 143 of Progress in Mathematics, pages 317-352. Birkhäuser, 1996.

[78] Sylvain Petitjean, Jean Ponce, and David J. Kriegman. Computing Exact Aspect Graphs of Curved Objects: Algebraic Surfaces. International Journal of Computer Vision, 9(3):231-255, 1992.

[79] Ragni Piene. Numerical Characters of a Curve in Projective $n$-Space. In P. Holm, editor, Real and Complex Singularities, Oslo, pages 475-495, 1976.

[80] Ragni Piene. Polar Classes of Singular Varieties. Annales Scientifiques de l'École Normale Supérieure, 11:247-276, 1978.

[81] Ragni Piene. Some Formulas for a Surface in $\mathbb{P}^{3}$. In L.D. Olson, editor, Algebraic geometry (Proc. Sympos., Univ. Tromsø, Tromsø, 1977), volume 687 of Lecture Notes in Mathematics, pages 196-235. Springer, 1978.

[82] Ragni Piene. Cuspidal Projections of Space Curves. Mathematische Annalen, 256:95-119, 1981.

[83] O.A. Platonova. Projections of Smooth Surfaces. Journal of Mathematical Sciences, 35(6):2796-2808, 1986.

[84] Jean Ponce and Martial Hebert. On Image Contours of Projective Shapes. In European Conference on Computer Vision, pages 736-749. Springer, 2014.

[85] Jean Ponce and David J. Kriegman. Computing Exact Aspect Graphs of Curved Objects: Parametric Surfaces. Department of Computer Science, University of Illinois at Urbana-Champaign, 1990.

[86] Jean Ponce, Bernd Sturmfels, and Matthew Trager. Congruences and Concurrent Lines in Multi-View Geometry. Advances in Applied Mathematics, 88:62-91, 2017. 
[87] Ziv Ran. Surfaces of Order 1 in Grassmannians. Journal für die reine und angewandte Mathematik, 368:119-126, 1986.

[88] Kristian Ranestad and Bernd Sturmfels. On the Convex Hull of a Space Curve. Advances in Geometry, 12:157-178, 2012.

[89] Ramon Ricker and Walt Weiskopf. The Augmented Scale in Jazz: A Player's Guide. Jamey Aebersold, 2010.

[90] Joachim H. Rieger. Global Bifurcation Sets and Stable Projections of Nonsingular Algebraic Surfaces. International Journal of Computer Vision, 7(3):171-194, 1992.

[91] Joachim H. Rieger. Computing View Graphs of Algebraic Surfaces. Journal of Symbolic Computation, 16(3):259-272, 1993.

[92] L. Roth. Line Congruences in Three Dimensions. Proceedings of the London Mathematical Society, s2-32:72-86, 1931.

[93] Leonard Roth and John G. Semple. Introduction to Algebraic Geometry. Clarendon Press, Oxford, 1949.

[94] George Salmon. A Treatise on the Analytic Geometry of Three Dimensions. Hodges, Figgis, \& Co., 4 edition, 1882.

[95] Robert Schumacher. Classification der algebraischen Strahlensysteme. Mathematische Annalen, 37:100-140, 1890.

[96] A. Seidenberg. Comments on Lefschetz's Principle. The American Mathematical Monthly, 65(9):685-690, 1958.

[97] Anna Seigal and Bernd Sturmfels. Real Rank Two Geometry. Journal of Algebra, 484:310-333, 2017.

[98] Aron Simis, Bernd Ulrich, and Wolmer V. Vasconcelos. Tangent Star Cones. Journal für die reine und angewandte Mathematik, 483:23-59, 1997.

[99] Bernd Sturmfels. Algorithms in Invariant Theory. Springer Science \& Business Media, 2008.

[100] Bernd Sturmfels. Fitness, Apprenticeship, and Polynomials. In G.G. Smith and B. Sturmfels, editors, Combinatorial Algebraic Geometry, volume 80 of Fields Institute Communications, pages 1-19. Springer, 2017.

[101] Bernd Sturmfels. The Hurwitz Form of a Projective Variety. Journal of Symbolic Computation, 79:186-196, 2017.

[102] Zach Teitler. An Informal Introduction to Computing with Chern Classes. Available at works . bepress.com/zach_teitler/2/, 2004. 
[103] Evgueni A. Tevelev. Projective Duality and Homogeneous Spaces, volume 133 of Encyclopaedia of Mathematical Sciences. Springer, 2005.

[104] René Thom. Structural Stability and Morphogenesis. W.A. Benjamin, 1975.

[105] Bartel L. van der Waerden. Zur algebraischen Geometrie II. Mathematische Annalen, 108:253-259, 1933.

[106] Walt Weiskopf. Understanding the Diminished Scale: A Guide for the Modern Jazz Player. Jamey Aebersold, 2015. 


\section{List of Figures}

3.1 The degree of the Chow hypersurface of a space curve $C$ is $\operatorname{deg} C$. $\quad 24$

3.2 This matrix has rank $\leq 2$ if and only if the quadric given by $c$ is

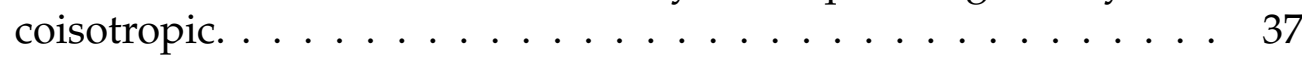

5.1 The class of the secant congruence of a space curve. . . . . . . . . 66

5.2 A bitangent and an inflectional line corresponding to a node and a cusp of the dual curve. $\ldots \ldots \ldots \ldots 70$

5.3 The degree of the Hurwitz hypersurface of a surface. . . . . . . . 71

5.4 Polar curve. . . . . . . . . . . . . . . 72

5.5 A secant projecting onto a node and a tangent projecting to a cusp. 72

$6.1 \quad$ Duality relations of a developable surface $\mathcal{S}$. . . . . . . . . . . . 93 6.2 Loci of planes and lines that meet a curve $X$ with assigned mul-

6.3 Loci of planes and lines that meet a surface $X$ with assigned mul-

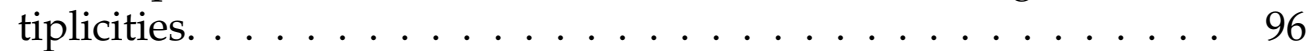

6.4 Changing views of a curve correspond to Reidemeister moves. The viewpoint $z$ crosses the tangential surface (left), edge surface (middle), or trisecant surface (right). . . . . . . . . . . . . 97

6.5 The catalogue of visual events for the projections of a smooth surface from a viewpoint that moves. The local events (left, from top to bottom) are lip, beak-to-beak, swallowtail. The multi-local events (right, from top to bottom) are tangent crossing, cusp crossing, triple point. Reprinted from [76] with permission of Springer. . . . . . . . . . . . . 111

6.6 Commutative diagram of some morphisms appearing in this section. Classes in the Chow rings of the depicted varieties are represented in gray. . . . . . . . . . . . . . . . 114

9.1 Western pitch space in modern times with twelve pitch classes. . 153

9.2 Cyclic order of the twelve pitch classes. Marked in green: $C$ major scale with interval sequence. . . . . . . . . . . . . . 155

$9.3 \mathcal{K}_{2} \ldots \ldots \ldots \ldots \ldots \ldots \ldots \ldots$

9.4 The three diminished scales. . . . . . . . . . . . . . . . . . 157

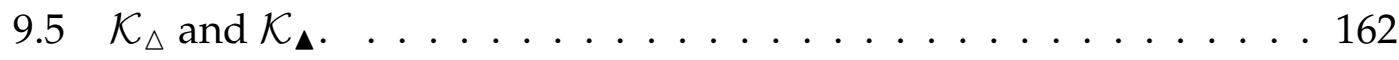


9.6 The left simplicial complex has 3 facets, but only one of these can be collapsed: $\{1,2,3\}$. In doing so, we can either remove the inner edge (green) or one of the two outer edges (e.g., orange). In the first case, we cannot perform a second collapse after the first one. In the second case, there is exactly one further collapse

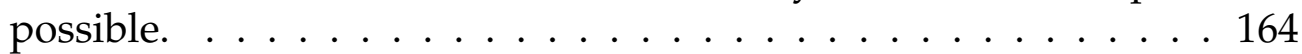

9.7 Messiaen's nine-note scale. . . . . . . . . . . . . . . . . . . . . 165

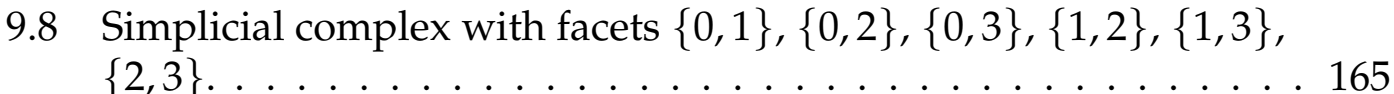

9.9 Schematic illustration of the topology of $\mathcal{K}_{N C} . \ldots \ldots 6$

9.10 The hexatonic facets of $\mathcal{K}_{N C}$ are the pairwise intersections of the four Messiaen scales. . . . . . . . . . . . . . . . 167 


\section{List of Tables}

4.1 Types of strongly coisotropic varieties associated to an irreducible variety $X$ in $\mathbb{P}^{n} . \ldots \ldots \ldots \ldots$. . . . . . . . . . . 52

6.1 Degrees of the components of the visual event surface of a space

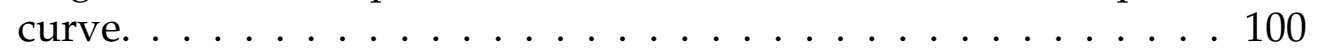

6.2 Degrees of the components of the visual event surface of a general surface. . . . . . . . . . . . . . . . 118

6.3 The ideals $\Delta_{(\lambda)}(d)$ of multiple root loci relevant for visual events of surfaces. . . . . . . . . . . . . . . . . . 120

$7.1 \quad$ All cases of isotropic curves $\Sigma \subset \mathrm{Gr}\left(1, \mathbb{P}^{3}\right)$. The developable surface $\mathcal{S}$ is ruled by the lines on $\Sigma$. See also Figure 6.1 . . . . . . . 130

7.2 All types of congruences in $\operatorname{Gr}\left(1, \mathbb{P}^{3}\right)$. The value in the column Seg-type denotes $\mid \mathbb{P}\left(T_{\Sigma, L}\right) \cap \operatorname{Seg}\left(\boldsymbol{L}, \mathbb{A}^{4} / \mathbf{L}\right)$ for a general $L$ in the congruence $\Sigma . \ldots \ldots 135$

9.157 maximal non-chromatic scales. . . . . . . . . . . . . . . . 157 



\section{List of Symbols}

\begin{tabular}{|c|c|}
\hline$Z(P)$ & zero locus in affine or projective space of a set $P$ of polynomials \\
\hline$I(S)$ & vanishing ideal of a subset $S$ of affine or projective space \\
\hline $\mathbb{P}(\mathcal{C})$ & projectivization of an affine cone $\mathcal{C}$ \\
\hline$S$ & affine cone over a set $S$ in projective space \\
\hline $\bar{S}$ & Zariski closure of a subset $S$ of affine or projective space \\
\hline$V^{*}$ & dual space of a (vector or projective) space $V$ \\
\hline$X^{\vee}$ & projectively dual variety of a projective variety $X$ \\
\hline $\mathcal{N}_{X, X^{\vee}}$ & conormal variety of a projective variety $X$ \\
\hline $\operatorname{span}(S)$ & linear or projective span of a set $S$ of vectors \\
\hline $\operatorname{Hom}(U, W)$ & vector space of all homomorphisms from $U$ to $W$ \\
\hline$\varphi^{*}$ & dual homomorphism in $\operatorname{Hom}\left(W^{*}, U^{*}\right)$ of $\varphi \in \operatorname{Hom}(U, W)$ \\
\hline$E_{\alpha}(u)$ & $\begin{array}{l}\alpha \text {-space of all } \varphi \in \operatorname{Hom}(U, W) \text { with } u \subset \operatorname{ker} \varphi \text {, where } u \subset U \text { is a } \\
\text { linear hyperplane }\end{array}$ \\
\hline$E_{\beta}(w)$ & $\begin{array}{l}\beta \text {-space of all } \varphi \in \operatorname{Hom}(U, W) \text { with } \operatorname{im} \varphi \subset w \text {, where } w \subset W \text { is a } \\
\text { one-dimensional linear subspace }\end{array}$ \\
\hline$(\varphi \bmod V)$ & $\begin{array}{l}\text { composition of the canonical projection } V_{1} / V_{2} \rightarrow V_{1} / V \text { with } \\
\varphi \in \operatorname{Hom}\left(U, V_{1} / V_{2}\right) \text { if } V_{2} \subset V \subset V_{1}\end{array}$ \\
\hline $\operatorname{Seg}(U, W)$ & Segre variety of all rank one homomorphisms in $\mathbb{P}(\operatorname{Hom}(U, W))$ \\
\hline $\operatorname{Gr}(\ell, V)$ & $\begin{array}{l}\text { Grassmannian of all } \ell \text {-dimensional subspaces of a (vector or } \\
\text { projective) space } V\end{array}$ \\
\hline$p_{i_{1} \ldots i_{n-\ell}}(L)$ & primal Plücker coordinates of a line $L \in \operatorname{Gr}\left(\ell, \mathbb{P}^{n}\right)$ \\
\hline$q_{j_{0} \ldots j_{\ell}}(L)$ & dual Plücker coordinates of a line $L \in \operatorname{Gr}\left(\ell, \mathbb{P}^{n}\right)$ \\
\hline$\Sigma^{\perp}$ & $\begin{array}{l}\text { image of a subset } \Sigma \subset \operatorname{Gr}\left(\ell, \mathbb{P}^{n}\right) \text { under the canonical isomorphism } \\
\operatorname{Gr}\left(\ell, \mathbb{P}^{n}\right) \cong \operatorname{Gr}\left(n-\ell-1,\left(\mathbb{P}^{n}\right)^{*}\right)\end{array}$ \\
\hline $\operatorname{Reg}(X)$ & smooth locus of a variety $X$ \\
\hline $\operatorname{Sing}(X)$ & singular locus of a variety $X$ \\
\hline$T_{X, x}$ & tangent space of a variety $X$ at $x \in \operatorname{Reg}(X)$ \\
\hline $\mathbb{T}_{X, x}$ & $\begin{array}{l}\text { embedded projective tangent space of a projective variety } X \text { at } \\
x \in \operatorname{Reg}(X)\end{array}$ \\
\hline$N_{X, x}$ & normal space of a subvariety $X$ of a Grassmannian at $x \in \operatorname{Reg}(X)$ \\
\hline$N_{X, x}^{*}$ & conormal space of a subvariety $X$ of a Grassmannian at $x \in \operatorname{Reg}(X)$ \\
\hline $\mathrm{CH}_{i}(X)$ & $i$-th coisotropic variety of a projective variety $X$ \\
\hline$P_{i}(X, V)$ & $\begin{array}{l}i \text {-th polar variety of a variety } X \subset \mathbb{P}^{n} \text { with respect to a subspace } \\
V \subset \mathbb{P}^{n}\end{array}$ \\
\hline $\mathcal{G}_{\ell}(X)$ & $\begin{array}{l}\ell \text {-th associated variety of a variety } X \subset \mathbb{P}^{n} \text { consisting of all } \\
L \in \operatorname{Gr}\left(\ell, \mathbb{P}^{n}\right) \text { intersecting } X\end{array}$ \\
\hline $\operatorname{Sec}_{k}^{0}(X)$ & $\left\{L \in \operatorname{Gr}\left(k, \mathbb{P}^{n}\right) \mid L=\operatorname{span}(L \cap X)\right\}$ for a variety $X \subset \mathbb{P}^{n}$ \\
\hline
\end{tabular}


$\operatorname{Sec}_{k}(X) \quad$ Zariski closure of $\operatorname{Sec}_{k}^{0}(X)$

$\operatorname{Sec}(X) \quad$ short for $\operatorname{Sec}_{1}(X)$

$\operatorname{Bit}(X) \quad$ Zariski closure of the set of all bitangent lines of a hypersurface $X \subset \mathbb{P}^{n}$

$\mathcal{L}_{m}(X) \quad$ Zariski closure of the set of all lines which intersect a

$\operatorname{Infl}(X) \quad$ synonym for $\mathcal{L}_{3}(X)$

$\operatorname{Osc}_{k}(C) \quad$ the curve in $\operatorname{Gr}\left(k, \mathbb{P}^{n}\right)$ of osculating $k$-planes of a curve $C$ in $\mathbb{P}^{n}$ $\mathcal{T}^{\ell}(C) \quad$ short for $\mathrm{Osc}_{1}(C)$

$\mathcal{T}(\mathrm{C}) \quad$ tangential surface of a curve $C$, i.e., ruled by lines on $\mathcal{T}^{\ell}(C)$

$A^{c}(X) \quad$ Chow group of codimension-c cycles on a variety $X$

$A^{*}(X) \quad$ Chow ring of a smooth projective variety $X$

$[Y]$

$c_{i}(\mathcal{E})$ rational equivalence class in $A^{*}(X)$ of a subvariety $Y \subset X$

$\mathcal{V}(X)$ $i$-th Chern class in $A^{i}(X)$ of a vector bundle $\mathcal{E}$ on a variety $X$ visual event surface of a curve or surface $X \subset \mathbb{P}^{3}$ 